\title{
Excitation-contraction coupling in small arteries: role in hypertension
}

Citation for published version (APA):

Boonen, H. C. M. (1992). Excitation-contraction coupling in small arteries: role in hypertension. [Doctoral Thesis, Maastricht University]. Datawyse / Universitaire Pers Maastricht.

https://doi.org/10.26481/dis.19921218hb

Document status and date:

Published: 01/01/1992

DOI:

10.26481/dis.19921218hb

Document Version:

Publisher's PDF, also known as Version of record

\section{Please check the document version of this publication:}

- A submitted manuscript is the version of the article upon submission and before peer-review. There can be important differences between the submitted version and the official published version of record.

People interested in the research are advised to contact the author for the final version of the publication, or visit the DOI to the publisher's website.

- The final author version and the galley proof are versions of the publication after peer review.

- The final published version features the final layout of the paper including the volume, issue and page numbers.

Link to publication

\footnotetext{
General rights rights.

- You may freely distribute the URL identifying the publication in the public portal. please follow below link for the End User Agreement:

www.umlib.nl/taverne-license

Take down policy

If you believe that this document breaches copyright please contact us at:

repository@maastrichtuniversity.nl

providing details and we will investigate your claim.
}

Copyright and moral rights for the publications made accessible in the public portal are retained by the authors and/or other copyright owners and it is a condition of accessing publications that users recognise and abide by the legal requirements associated with these

- Users may download and print one copy of any publication from the public portal for the purpose of private study or research.

- You may not further distribute the material or use it for any profit-making activity or commercial gain

If the publication is distributed under the terms of Article $25 \mathrm{fa}$ of the Dutch Copyright Act, indicated by the "Taverne" license above, 


\section{EXCITATION-CONTRACTION COUPLING IN SMALL ARTERIES: ROLE IN HYPERTENSION}


CIP-GEGEVENS KONINKLIJKE BIBLIOTHEEK, DEN HAAC

Boonen, Henricus Comelis Matjeu

Excitation-contraction coupling in small arteries: role in hypertension / Henricus Cornelis Matjeu Boonen. -

Maastricht : Universitaire pers Maastricht. - Ill.

Thesis Maastricht. - With ref.

ISBN 90-5278-040-4

NUGI 743

Subject headings: hypertension ; pharmacology /

excitation-contraction coupling; small arteries. 


\title{
EXCITATION-CONTRACTION COUPLING IN SMALL ARTERIES:
}

\author{
role in hypertension
}

\section{PROEFSCHRIFT}

ter verkrijging van de graad van doctor aan de Rijksuniversiteit Limburg te Maastricht, op gezag van Rector Magnificus, Prof. Mr. M.J. Cohen, volgens besluit van het College van Dekanen, in het openbaar te verdedigen op vrijdag, 18 december 1992 om 12.00 uur

door

Henricus Cornelis Matjeu Boonen

geboren te Buggenum in 1964

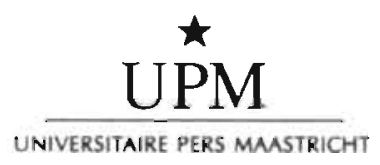




\section{Promotor}

Prof. dr. H.A.J. Struijker Boudier

\section{Co-promotor}

Dr. J.G.R. De Mey

\section{Beoordelingscommissie}

Prof. dr. P.W. De Leeuw, voorzitter

Prof. dr. M.A. Allessie

Prof. dr. M.J. Mulvany, University of Aarhus, Denmark

Prof. dr. R.F.A. Zwaal

Prof. dr. P.A. van Zwieten, Universiteit van Amsterdam

Production: Datawyse I Universitaire Pers Maastricht

This thesis was supported, in part, by funds from the Royal Netherlands Acadamy of Sciences (KNAW) and the Netherlands Organisation for Scientific Research (NWO).

Financial support by the Netherlands Heart Foundation for the publication of this thesis is gratefully acknowledged

For financial support for the publication of this thesis we thank:

- Astra Pharmaceuticals BV, the Netherlands and

- J.P. Trading Corp., Science Park, Aarhus, Denmark 
Veur Papa en moe Til Susanne 


\section{CONTENTS}

1. Introduction

2. Material and methods 21

3. $\quad \alpha_{1}$-Adrenoceptor subtype population and calcium antagonist 35 efficacy in large and small mesenteric arteries of the rat

4. Increased calcium sensitivity in isolated resistance arteries from spontaneously hypertensive rats: effects of dihydropyridines

5. G-Proteins are involved in contractile responses of isolated mesenteric resistance arteries to agonists

6. Effects of a phorbol ester and staurosporine on electro- and pharmacomechanical coupling in a resistance artery

7. Length-dependency of excitation-contraction coupling in isolated mesenteric resistance arteries

8. Distension sensitises small artery smooth muscle to vasoconstrictors and to intracellular calcium

9. Loss of hypersensitivity to vasoconstrictors in mesenteric muscular and resistance arteries of spontaneously hypertensive rats following tissue culture

10. Resistance artery changes following vasoconstrictor infusion in young rats. Comparison with those in young spontaneously hypertensive rats

11. General discussion

References

Summary

Samenvatting

Curriculum Vitae

List of Publications

Dankwoord 


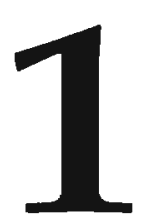

Introduction 
Hypertension is a condition that predisposes for secondary pathologies such as myocardial infarction and stroke. These account for a major part of human mortality. Small arteries play a pivotal role in the control of blood pressure and in the development of hypertension. They contribute to the resistance to blood flow through their ability to constrict or dilate and through their overall structure. Yet, knowledge of their function is primarily based on observations obtained in large arteries and in non-vascular smooth muscle preparations. Evidence accumulated during the past 15 years indicated, however, that these may not be representative for "resistance arteries". To understand the aetiology of hypertension it is therefore of basic importance to directly study the contractile reactivity of small arteries. This depends on molecular events that take place at the cell membrane and in the cytoplasm of the smooth muscle cells that populate the wall of small arteries. Furthermore, integration of cellular mechanisms with those that take place on a higher level such as intercellular communication, neuroendocrine mechanisms and mechanical forces, is essential for a better understanding and treatment of hypertension.

\section{THE VASCULAR SYSTEM}

\section{Physiological function}

The physiological function of the vasculature is to distribute blood to and collect blood from the different organs to assure optimal exchange of nutrients, oxygen and metabolites from the tissue. From the heart the aorta branches into several large arteries that feed different organs or regions within the body. These major arteries branch progressively into smaller arteries forming an arterial tree. Exchange of oxygen and nutrients and control of fluid balance. in the body is finally accomplished by a dense network of capillaries which consist merely of an endothelial layer and a basal lamina. From the capillaries, the blood is collected into small venules which gather into veins. These in turn end in the vena cava that returns the blood to the right atrium of the heart. Subsequently the blood is pumped into the pulmonary circulation to be resaturated with oxygen and depleted of carbondioxide.

Large arteries act as elastic reservoirs or capacitors, distending during systole and reducing their diameter during diastole which results in a damping of the pulse pressure difference and a reduction in pulsatile flow of the blood. Muscular arteries distribute the blood over different organs. Smaller arteries are increasingly responsible for local distribution of blood flow in an organ. To assure an adequate supply of blood, flow through different vascular beds is regulated. The pressure gradient $(\Delta \mathrm{P})$ that drives the blood through the arterial tree is of relevance for the blood flow $(\mathrm{Q})$. However, also the resistance (R) to blood flow that becomes manifest in increasingly smaller "tubes" is important. This relation can be expressed in the following equation: 


$$
Q=\frac{\Delta P}{R}
$$

The resistance of the vascular system (total peripheral resistance) or local resistance of different vascular beds can be altered dramatically through narrowing or widening of the diameter of the summated vasculature in the bed. A. measure of resistance may be obtained by rearranging the Poiseuille equation to give,

$$
R=\sum_{i=1}^{n} \frac{1}{r_{i}^{4}} \cdot l_{i} \cdot \eta \cdot \frac{8}{\pi}
$$

in which $r$ represents the sum of the internal radii of the total number $(n)$ of series coupled vessels, 1 the sum of the total vessel lengths and $\eta$, the viscosity of the blood. From the above it will be clear that regulation of vessel diameter has powerful consequences for blood flow and pressure, since radius contributes to the fourth power in Poiseuille's equation.

Small arteries are therefore assumed to be increasingly involved in the resistance to blood flow. Different approaches have been used to localise the exact site of major resistance within the vascular tree. A pressure-resistance relationship can be drawn by measuring intra-arterial pressure drop along the arterial tree (Bohlen, 1986; Folkow, 1990). Borders and Granger (1986), calculated the dissipation of power along the arterial tree to localise major resistance sites. Although these studies indicate that pressure-resistance relations depend very much on the vascular bed studied (see Bohlen and Aukland, 1991), they also indicate that a significant fall in intra-arterial pressure occurs already in arteries with diameters of 400-200 $\mu \mathrm{m}$ (Mellander, 1989; Mulvany and Aalkjaer, 1990; Bohlen and Aukland, 1991).

\section{Regulation of arterial smooth muscle function}

On the basis of histologically defined criteria the arterial wall can be divided into three major layers. From the luminal to the abluminal side these comprise of the tunica intima, the tunica media and the tunica adventitia. These layers are usually separated by an elastic membrane, the lamina elastica interna and externa, respectively. The intima consists mainly of a basal membrane and thin endothelial cells that line up the lumen of the vessel. The medial layer contains smooth muscle cells that are normally oriented helically around the lumen of the vessel. The extracellular matrix of the media, which is synthesised by the smooth muscle cells, consists predominately of collagen and elastic fibres. The adventitia is mainly comprised of connective tissue synthesised by sparsely seeded fibroblasts. Nerve endings of primarily sympathetic origin are present at the adventitial side of the media. These structures can affect the function of the vessel wall either in an active or a passive way. 
local control of vascular function

It: was shown that blood flow, vascular diameter and pressure are closely related to each other, implying that for flow and pressure to remain within physiological ranges, carefully balanced vascular diameters are required. First of all this is achieved by a certain tone that is present in vivo in all arteries. This arterial tone is the net result of interacting local and remote modulatory systems (Fig 1.1). Depending on tissue requirements these systems act to delicately tune blood flow that is feeding the perfused tissue.

\section{REMOTE CONTROL}

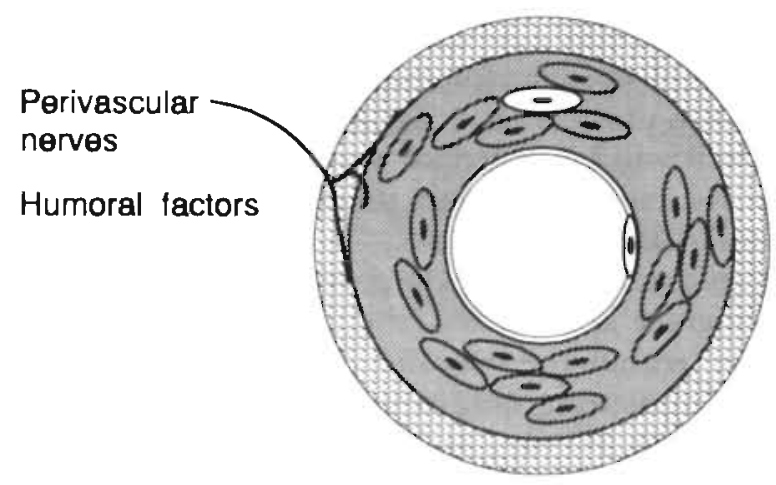

LOCAL CONTROL

\author{
Endothelium \\ Mechanical factors \\ Metabolic factors
}

Figure 1.1. Schematic cross-section of a vascular wall in which different smooth muscle regulatory mechanisms are displayed. Control of vessel tone is achieved by an interplay of local regulators such as the endothelium, tissue metabolites, mechanical factors and remote mechanisms such as nerves and hormones.

A basal tone is present in arteries. This tone originates within the smooth muscie celis without neurogenic or endothelial interference and is therefore referred to as myogenic tone (Fig. 1.1). Pressure changes may affect myogenic tone (Mellander, 1989; Meininger et al., 1991; Messing et al., 1991). It has been shown that an elevation of pressure in vivo or in vitro results in an active narrowing of the lumen of the vessel keeping flow constant. This phenomenon was observed already in the beginning of this century by Bayliss (1902) and has since been called the myogenic response. This autoregulatory mechanism is capable within a physiological pressure range, to prevent hyper- or hypoperfusion of the downstream capillary network (Johnson, 1980; Mellander, 1989). The mechanisms that lead to a myogenic response in vascular smooth muscle following increa- 
ses in intravascular pressure are not fully known at present. Myogenic tone and autoregulatory responsiveness do not always suffice to regulate tissue requirements. Therefore, superimposed on the myogenic tone, other mechanisms exert their regulatory role on vessel diameter.

Endothelial cells have multiple modulatory effects on vascular smooth muscle cell function. Furchgott and Zawadski (1980) were the first to describe a vasodilator action of acetylcholine on smooth muscle cells that depended on the presence of the endothelium. The vasodilating substance that was released from the endothelium by stimulation with acetylcholine was referred to as endothelium derived relaxing factor (EDRF). Only years after the discovery of EDRF it was found that nitric oxide which is produced by endothelial cells after stimulation of for example muscarinic receptors from the aminoacid 1arginine, had similar properties as EDRF (Palmer et al., 1988; Moncada et al., 1989). It is therefore believed that nitric oxide or a substance that can generate nitric oxide embodies the endothelium derived relaxing factor (Palmer et al., 1988; Moncada et al., 1989). Besides inhibitory actions through the release of EDRF, the endothelium has also been shown to either stimulate or inhibit the responsiveness of vascular smooth muscle through the release of other contractile or relaxing substances or through effects on vascular smooth muscle membrane potential (D'Orleans-Juste et al., 1985; Yanagisawa et al., 1988; Vanhoutte, 1989; Egleme et al., 1990; Kato et al., 1990). The effect of the endothelium on vascular smooth muscle cells often depends on haemodynamic factors such as blood flow, pressure or shear stress (Miller and Vanhoutte, 1988; Hutcheson and Griffith, 1991).

Local control of vascular reactivity may also be mediated through smooth muscle cells. Smooth muscle cells within the media of vessels have been shown to act as an electrical syncytium (Duling et al., 1991; Segal, 1991). Contractile or vasodilatory responses that are initiated at a certain location in a microcirculatory preparation can be electrically conducted along the wall of arterioles in either direction, probably through gap-junctions that interconnect neighbouring smooth muscle cells (Duling et al., 1991). Whether this means of communication between smooth muscle cells can also be observed in small arteries and, if so, how it would affect vessel responsiveness has not been fully elucidated yet.

\section{Remote control of vascular function}

From the other side of the tunica media, vascular smooth muscle function is regulated by the action of sympathetic and peptidergic nerves (Fig. 1.1) (Hirst and Edwards, 1989). Following stimulation of sympathetic nerves, noradrenaline, the purine ATP and neuropeptide $Y$ can be released into the synaptic cleft between nerve and smooth muscle cell membrane (Hirst and Edwards, 1989; Lundberg et al., 1990). The sympathetic neurotransmitter noradrenaline may have different postsynaptic effects on vascular smooth muscle but can also influence its own release and neuronal uptake through stimulation of presynaptic $\alpha_{2}$-adrenoceptors (Rump et al., 1991).

Through activation of different postsynaptic adrenoceptor subtypes by noradrenaline, either relaxing or contractile responses can be induced in vas- 
cular smooth muscle. $\alpha_{1}$ - and $\alpha_{2}$-adrenoceptors can be distinguished according to their selectivity for certain agonists, antagonists and differences in calcium mobilising pathways (De Mey and Vanhoutte, 1981; Korstanje and van Zwieten, 1987; Raymond et al., 1990). Stimulation of $\alpha$-adrenoceptors leads to vasoconstriction of vascular smooth muscle (for review see: Raymond et al., 1990). A further subclassification of $\alpha_{1}$ - and $\alpha_{2}$-adrenoceptors on vascular smooth muscle cells has complicated the understanding of their actual physiological function. $\alpha_{1 A^{-}}$and $\alpha_{1 B^{-}}$ adrenoceptors, for example, differ with respect to their relative affinity for either WB 4101, the non-competitive antagonist chloroethylclonidine and specific signal transduction pathways (Han et al., 1987; Daly et al., 1990; Muramatsu et al., 1990; Suzuki et al., 1990; Wilson and Minneman, 1990; Oriowo and Ruffolo, 1992). The distribution of various $\alpha$-adrenoceptor subtypes appears to be heterogenous throughout the vascular tree (De Mey and Vanhoutte, 1981; Daly et al., 1988; Faber, 1988; Heesen and De Mey, 1990; Messing et al., 1990; Nielsen et al., 1992). Which $\alpha$-adrenoceptor subtypes are present on the resistance arterial level and how these may affect responsiveness to adrenergic agonists requires more detailed investigation.

Adrenergic vasorelaxing responses are exerted through stimulation of $\beta$ adrenoceptors. Relaxing responses induced by $\beta$-adrenoceptor activation are mediated through the generation of cyclic adenosine monophosphate (cAMP) (Raymond et al., 1990). The additional hyperpolarising action of $\beta$-adrenoceptor activation (Mulvany et al., 1982), may act in concert with the vasodilator effect of increased cAMP concentrations in vascular smooth muscle.

Many other substances that do not directly originate from the endothelium or perivascular nerves may exert definite effects on vascular smooth muscle cell responsiveness. Among these are hormones (Grillone et al., 1988; Tabrichi and Triggle, 1991), autocoids (De Mey and Gray, 1985; Edwards, 1985; Lüscher and Vanhoutte, 1988) and tissue metabolites (De Mey et al., 1979; Silver et al., 1984). From the above, it can be concluded that vascular smooth muscle function is carefully controlled by a variety of means. On a lower level of organisation, however, vascular smooth muscle function depends on the operation of a complex machinery of biochemical processes, generally termed the contractile apparatus.

\section{CONTRACTION OF VASCULAR SMOOTH MUSCLE CELLS}

\section{The contractile machinery}

A common feature of muscle cells in general and vascular smooth muscle cells in particular is their ability to contract. Contraction or cellular shortening is mainly achieved by the interaction of two proteins, actin and myosin which are major constituents of the contractile apparatus. In principle these proteins consist of a variety of different isozymes found in skeletal, cardiac and smooth muscle but also in non-contracting cells (D'Orleans-Juste et al., 1985: Lusscher, 1990), implicating that some of these isozymes are non-contractile. 
Simplified, force development is achieved by cyclic high affinity cross-bridge formation of actin filaments with the larger myosin filament (for review see: Hai and Murphy, 1989; Hathaway et al., 1989). Contraction occurs by a translocation of myosin heads that bind to actin, resulting in a transaxial sliding of the filaments across each other. The energy for this mechanical work is mainly delivered by adenosine triphosphate (ATP) Johansson, 1987; Hai and Murphy, 1989). Myo$\sin$ and actin are in turn anchored in a network of numerous intermediate filaments and attachment proteins which form the cytoskeleton (Kocher et al, 1985: Rubbia and Gabbiani, 1989; Watson, 1991). The cytoskeleton in turn has connections to cell membrane and extracellular matrix.

Several enzymes delicately control the process of cross-bridge formation between actin and myosin. The myosin light chain kinase (MLCK) is an enzyme that, provided it has been activated by the calcium binding protein calmodulin, phosphorylates the myosin light chain rendering it the ability to bind actin with high affinity (Hai and Murphy, 1989). Cross-bridge formation is counterregulated by a myosin light chain phosphatase (MLCP) which de-phosphorylates the myosin light chain (Hai and Murphy, 1989). Maintenance of contraction in smooth muscle has been suggested to involve another state of interaction between actin and myosin, the so called "latch state" which implies that both proteins bind more tightly to one another without the necessity of energy consuming cycling. This state of interaction between actin and myosin has a lower calcium dependence (Murphy, 1978; Hai and Murphy, 1989; Rembold, 1991). The kinase and phosphatase activities are in turn regulated by other enzymes that usually require the presence of free calcium cations. A calcium dependent protein that activates MLCK in vascular smooth muscle is calmodulin which has a function analogous to the skeletal muscle troponin-C (Hai and Murphy, 1989; Hathaway et al., 1989; Kamm and Stull, 1989). More recently other proteins have been identified that may play an important role in the regulation of the contractile apparatus. Caldesmon is found in vascular smooth muscle co-existing with the thin actin filaments (Hai and Murphy, 1989; Kamm and Stull, 1989). Calponin, which has analogies with skeletal muscle troponin- $T$ (Carrow and Calhoun, 1964) is assumed to be a component of the smooth muscle actin filament. The phosphorylation of this very effective substrate of protein kinase- $C$ is sensitive to the presence of calcium and calmodulin. It is therefore suggested that calponin is involved in the effects of protein kinase-C on smooth muscle contraction (Takahashi et al, 1988).

Contraction induced through activation of the MLCK pathway was originally thought to be proportional to the concentration of intracellular calcium. However, it is becoming evident that additional biochemical pathways are involved that could increase the sensitivity of the contractile apparatus for calcium or even function in the absence of calcium (Aqel et al., 1986; Nishimura et al., 1988; Himpens et al., 1990; Khalil and Van Breemen, 1990; Kitazawa et al., 1991). Mechanisms that affect the calcium sensitivity of the contractile apparatus are not well understood at present. Nevertheless, taken together it is evident that the functional contractile response of a vascular smooth muscle cell is the effect of a complex interplay between contractile proteins, kinases, phosphatases and cations such as calcium and magnesium. 


\section{Regulation of intracellular calcium}

To trigger and maintain the cascade of protein activation and deactivation that leads to a contractile response in vascular smooth muscle, it has long been recognised that the divalent calcium ion is an essential participant. The free concentration of calcium $\left(\left[\mathrm{Ca}^{2+}\right]\right.$ ) that is present in the cytosol of vascular smooth muscle cells governs for an important part the activity of the contractile apparatus. Calcium is present in millimolar concentrations in the extracellular compartment of higher organisms. The highly specialised biochemical pathways that induce a contractile response, however, operate at $\left[\mathrm{Ca}^{2+}\right]$ that are about ten thousand times lower than the extracellular $\left[\mathrm{Ca}^{2+}\right]$. Therefore, if similar calcium concentrations would exist in the intracellular environment, this would fully activate numerous calcium dependent biochemical processes which in the case of muscle cells, would lead to a tetanic contracture and cell death. To circumvent such a hazardous situation, the cell is equipped with several systems that remove calcium from the intracellular space. Calcium extrusion from the cell and calcium uptake into intracellular stores enable the cell to preserve a $\left[\mathrm{Ca}^{2+}\right]_{i}$ that is below the threshold for activation of calcium dependent biochemical reactions. On the other hand, to meet its physiological function, the vascular smooth muscle cell is in need of mechanisms that are able to increase $\left[\mathrm{Ca}^{2+}\right]_{i}$ to higher levels to enable calcium dependent processes, such as the contractile response, to be activated.

\section{Calcium channels}

Due to the large difference in extracellular and intracellular concentrations, calcium leaks into the cell through non-specific pores in the cell membrane. However, besides this stationary, functionally relatively negligible leak of calcium the vascular smooth muscle cell is equipped with highly selective calcium channels. Their function can be modulated through activation by specific mechanisms. Activation leads to the influx of calcium ions along the electro-chemical gradient. Because of their involvement in the regulation of the contractile response in smooth muscle, these channels have generated considerable interest in and exploration of their operation.

In view of differences in their pharmacological properties and structural characteristics, calcium channels have classically been divided into several subgroups (Hurwitz; 1986; Van Breemen et al., 1986; Bolton et al., 1988). Calcium channels have been described that are opened by changes in membrane potential (Fig 1.2), following receptor stimulation (Fig. 1.3) and through physical forces applied to the cell, such as strain or stress. The so called potential- or voltageoperated calcium channels (VOC) have been studied most extensively. The patch clamp technique showed the presence in vascular smooth muscle cells of two types of voltage dependent channels (Bean et al., 1985; Loirand et al., 1986; Sturek and Hermsneyer, 1986). According to their kinetics they were termed T- and L-type voltage operated calcium channels (Fig. 1.2). T-type channels (transient-type) activate at quite negative membrane potentials and inactivate rapidly (Yatani et al., 1987; Bean, 1989). Their functional role in the regulation of intracellular calcium 


\section{INTRODUCTION}

is not well understood but they are thought to be involved in initiating calcium dependent processes in the cell (Yatani et al., 1987; Bean, 1989). On the other hand, Ltype voltage channels (long lasting-type) are calcium selective channels that activate at more positive membrane potentials and inactivate slowly (Bean, 1989). With the use of highly selective radiolabelled ligands, these channels have been isolated and cloned from different types of muscle cells. Even though their full chemical structure is largely known at present (Hosey and Lazdunski, 1988; Glossman. et al., 1989), many aspects of the function of these channels are still under intense investigation. Other types of voltage operated calcium channels have been identified on neural ( $N$-type) tissue and on Purkinje cells (P-type) but these have not been identified in vascular smooth muscle and will therefore not be discussed in the present dissertation (for review see: Bean, 1989).

Pharmacological studies suggested that calcium influx pathways exist that are not dependent on changes in membrane potential (Rüegg et al., 1989). Secondly, contractions evoked by full depolarisation of the cell membrane could be further increased by receptor activation (Cauvin et al., 1988). Furthermore, studies in which calcium influx in vascular smooth muscle was assessed indicate that the calcium flux induced by depolarisation could be further enhanced by receptor stimulation thus indicating additional influx pathways not: dependent on membrane potential (Van Breemen et al., 1972; Cauvin et al., 1987). Accordingly, these channels were referred to as receptor operated calcium channels (ROC) (Somlyo and Somlyo, 1968; Godfraind and Kaba, 1972; Van Breemen et al., 1972; Droogmans et al., 1977; Meldolesi and Pozzan, 1987; Rüegg et al., 1989). Some electrophysiological. observations suggest the presence of receptor operated calcium channels in non-vascular smooth muscle (Benham and Tsien, 1987; Sasaki and Gallacher, 1990). Opening of these channels through stimulation with ATP leads to the influx of calcium. They are however rather nonselective; sodium currents also increase significantly following channel opening (Benham and Tsien, 1987). Presently, one has not been able to isolate receptor operated calcium channels from vascular smooth muscle because of the lack of specific ligands. Other receptor operated calcium channels, which can be activated through binding of a ligand at a specific receptor site, are those that are found in the sarcoplasmic reticular membrane. These calcium channels can be opened following activation by inositol triphosphate and are therefore referred to as second messenger operated calcium channels (Putney, 1987).

A third type of calcium channel was proposed in vascular smooth muscle which was neither affected by changes in membrane potential nor by receptor stimulation. These channels appeared to be activated by physical deformation of the cell or cell membrane by stretch (Hwa and Bevan, 1986; Kirber et al., 1988; Laher and Bevan, 1989).

\section{Calcium exirusion}

Calcium is extruded from the cell primarily by a calcium -ATP-ase (Fig. 1.2) (Carafoli, 1987). This pump is an energy consuming calcium extrusion pathway. Besides active extrusion of calcium from vascular smooth muscle, it has been suggested that a pump exists that exchanges sodium for calcium (Carafoli, 
1987). This extrusion mechanism. (Fig 1.2) is driven mainly by the sodium gradient across the cell membrane. Depending on electrical and chemical gradients the exchanger will either extrude calcium from the cell or. lead to the influx of calcium (Carafoli, 1987). Whether the sodium-calcium exchanger contributes significantly to calcium extrusion in vascular smooth muscle is not fully understood.

Furthermore, besides pumps that extrude calcium from the intracellular space out of the cell, specific cell-organelles exist that can actively take up calcium from the cytosol. In principle this is achieved by a calcium ATP-ase that is similar to the one in the sarcolemma (Carafoli, 1987). Mitochondria are able to accumulate calcium but their role in vascular smooth muscle is small compared to the sarcoplasmic reticulum (Fig's. 1.2 and 1.3) which is capable of storing calcium in millimolar concentrations (Carafoli, 1987). The role of the sarcoplasmic reticulum as a regulator of intracellular calcium concentrations $\left(\left[\mathrm{Ca}^{2+}\right]_{i}\right)$ and contraction of vascular smooth muscle has been studied in great detail and was reviewed by several authors (Carafoli, 1987; Bers and Bridge, 1989). Because of their strategic position within the cell, in close proximity to both the cell membrane on one side and the contractile apparatus on the other, calcium stores are believed to function firstly as a buffer between the cell membrane and the contractile machinery for calcium leaking into the cell and secondly, as one of the key sites for the release of calcium into the cytosol (Saida and Van Breemen, 1984; Van Breemen and Saida, 1989).

\section{Calcium entry blockers}

Drugs have been developed that interfere with calcium channel function in either a stimulatory or inhibitory fashion. The different classes of calcium entry blockers or calcium antagonists that have been defined either by chemical structure, tissue selectivity or specificity have been reviewed in numerous publications (Fleckenstein, 1983; Godfraind et al., 1985; Struijker Boudier et al., 1990).

Inorganic cations can interact with calcium channels in a direct and reversible manner. Depending on their binding affinity for specific channel binding sites divalent cations can either pass the channel pore freely (barium and strontium), or will act as blockers of the channel (manganese, nickel and cobalt). Also trivalent cations, such as lanthanum, are potent inhibitors of calcium channels, and are therefore used experimentally for this purpose. However, except for nickel which is believed to be quite specific for ROC (Kass, et al., 1990), cations are rather nonspecific for the various types of calcium channels.

Organic calcium entry blockers are more selective and are used in the treatment of different cardiovascular pathologies (Fleckenstein et al., 1984, 1987; Struijker Boudier et al., 1990). The concept of calcium antagonism emerged in the sixties from studies with lidoflazine and cinnarizine and from studies in which it was shown that the inhibitory effect of verapamil on cardiac muscle contractility could be reversed by increasing the extracellular calcium concentration (Fleckenstein, 1964; Godiraind et al., 1968). Since, many organic calcium entry blockers with different therapeutic properties were developed. Based on their chemical structure and pharmacological properties, four major groups can be defined. 
The diphenylpiperazines, which include cinnarizine and flunarizine; the verapamil derivatives including gallopamil and tiapamil; the benzothiazepines of which diltiazem is an example and finally the dihydropyridines of which Bay K 8644, isradipine, felodipine, nifedipine, nimodipine and nitrendipine are some examples. Other substances that also have calcium antagonistic properties include: bepridil, prenylamine, papaverine and yohimbine derivatives.

The dihydropyridines encompass the largest and most diverse group of calcium entry blockers. Dihydropyridines have been found to have the highest. affinity and selectivity for the L-type calcium channel (Bean, 1989). For this reason, the L-type calcium channel has also been called the dihydropyridine receptor (Gill, 1989; Rampe and Triggle, 1989). T-type voltage operated calcium channels, on the other hand, are relatively insensitive to dihydropyridines (Bean, 1989). Of the different groups, dihydropyridines also display highest vascular selectivity (Fleckenstein et al., 1984; Lorimer et al., 1985; Ljung, 1990) and therefore prevail for the treatment of vascular related pathologies, whereas verapamil derivatives and diltiazem are preferably used as antiarrythmics (van Zwieten, 1989).

Some members of the dihydropyridine family have distinct properties from the rest of the group. Substances like Bay K 8644, Bay R 5417 and CGP 28392 have been shown to have calcium agonistic instead of antagonistic characteristics. These substances increase the opening of L-type calcium channels (Droogmans and Callewaert, 1986; Asano et al., 1987; Nelson et al., 1990), and have therefore been used as experimental drugs in the study of calcium channel function. Depending on the concentration used some dihydropyridines may exert agonistic or antagonistic properties (Schwartz, 1989; Prieto et al., 1991).

Already the fact that different types of calcium channels have been proposed, illustrates in a way that calcium channel function can be controlled by' different means. For the contractile apparatus present in the vascular smooth muscle cell to be controlled, signals from higher neural centres or hormone systems have to be transduced from the outside of the cell to the contractile filaments through these calcium channels. This process, which is also referred to as excitation-contraction coupling is the major mechanism regulating vascular smooth muscle function.

\section{Excitation-contraction coupling in vascular smooth muscle}

Generally, changes in the contractile state of the smooth muscle cell are regulated from the outside. This is achieved through a signal transduction mechanism that comprises the course of signals that are generated at the cell membrane surface to the intracellular space and contractile apparatus. Roughly three systems can be distinguished: electro-mechanical coupling, pharmacomechanical coupling and mechanotransduction (Fig's. 1.2 and 1.3).

\section{Electromechanical coupling}

Membrane potential governs the "activity" of voltage operated calcium channels. Thus, any intervention that changes membrane potential of the smooth muscle cell leads to either an opening or closure of voltage operated 


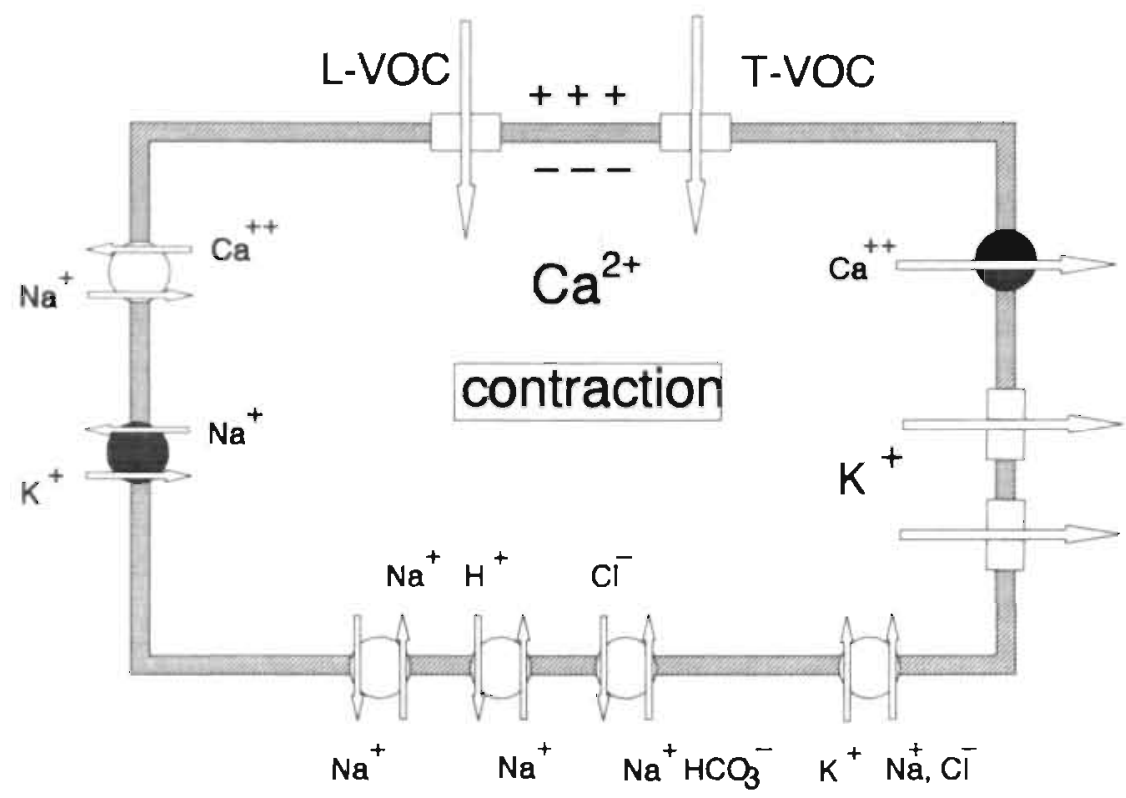

Figure 1.2. Electro-mechanical coupling mainly involves the stimulation of the contractile machinery by calcium that enters the cell through voltage operated calcium channels of different types ( $L-V O C$ and T-VOC, respectively, see text). These channels are sensitive to changes in membrane potential. Membrane potential is largely dependent on the permeability of the membrane for anions and cations and the activity of the Na" - K. ATPase. Membrane potential is significantly affected by the opening of specific potassium channels of which in vascular smooth muscle the calcium dependent $\left(\mathrm{K}_{\mathrm{CA}_{\mathrm{A}}}\right)$ and ATPsensitive $\left(\mathrm{K}^{*}{ }_{\mathrm{AT}}\right)$ channels have been identified. Various exchange mechanisms operate by virtue of ion-gradients across the cellmembrane $\left(\mathrm{Na}^{+}-\mathrm{Ca}^{2+}, \mathrm{Na}^{+}-\mathrm{H}^{*}, \mathrm{Na}{ }^{*}-\mathrm{Na}{ }^{*}, \mathrm{Na}^{*}\right.$. $\mathrm{HCO}_{1}$ exchange, $\mathrm{Cl}-\mathrm{HCO}_{3}, \mathrm{Na}^{*}-\mathrm{K}^{*}-\mathrm{Cl}^{-}$-cotransport). Calcium can be extruded from the cell by a $\mathrm{Ca}^{2 *}$-ATPase (energy consuming indicated by gray coloured circle) and by the exchange of sodium for calcium $\left(\mathrm{Na}^{-}-\mathrm{Ca}^{2 *}\right.$-exchange).

calcium channels. In principal, electromechanical coupling contracts smooth muscle on the basis of this phenomenon. The electrical potential difference across the cell membrane is the net result of the activity of a number of active and passive ion transport mechanisms in the cell membrane. Figure 1.2 illustrates some ion-pumps, exchange proteins and ion-channels that have been identified in vascular smooth muscle which can all affect membrane potential (Hermsmeyer, 1982, 1987; Mulvany and Aalkjaer, 1990). Of these, especially the electrogenic sodium pump $\left(\mathrm{Na}^{*} / \mathrm{K}^{+}\right.$-ATP-ase $)$and the different potassium channels (Weston and Abbot, 1987; Cook, 1988; Standen et al., 1989), can modify membrane potential in vascular smooth muscle (Hermsmeyer, 1982, 1987). The relationship between the opening of L-type voltage operated calcium channels and membrane potential has been reviewed in detail (Nelson et al., 1990). 
Mathematical models and experimental observations indicate that the probability of a calcium channel to be in an open state increases with less polarised membrane potential in a fashion that is much like the concentration dependent effects of contractile stimuli on vascular smooth muscle (Hirst and Edwards, 1989; Nelson et al., 1990). Within the physiological range of membrane potential in vascular smooth muscle, i.e. between about -45 and $-35 \mathrm{mV}$ (Neild and Keef, 1985; Hirst and Edwards, 1989; Nelson et al., 1990), the calcium channel's open time increases exponentially. This implies that within a narrow range of membrane potential, influx of calcium through L-type calcium channels changes dramatically. Depolarisation of vascular smooth muscle is therefore a very direct way of stimulating the contractile proteins.

\section{Pharmacomechanical coupling}

Binding of an agonist to its specific receptors on the cell membrane initiates a cascade of biochemical events leading to contraction or relaxation. Generally the pathway from agonist to cellular response consists of receptor activation, G-protein activation and subsequent second messenger generation resulting in activation or inhibition of the cellular response. Activation of a membrane bound receptor can lead to either direct stimulation of an intracellular messenger or to activation of so called G-proteins. G-proteins or GTP-binding regulatory proteins were first shown to be involved in the adenylate cyclase pathway by Rodbell in 1971 (see: Bimbaumer, 1990a). They have been shown to be membrane bound proteins consisting of three subunits. Receptor activation can induce the displacement of GDP by GTP on the $\alpha$-subunit thereby changing the conformational state of the protein and releasing the active binding site of the G-protein. The activated G-protein can in turn activate or inhibit enzymes or ion channeis through binding at specific target sites (Brown and Birnbaumer, 1988; Brown et al., 1989; Bimbaumer, 1990a). Second messenger systems that play a major role in the contractile function of vascular smooth muscle and that are regulated by G-proteins are the CAMP and the phosphatidylinositide pathways (Birnbaumer, 1990a, b). Briefiy, different G-proteins have been suggested in smooth muscle with respect to their stimulatory $\left(G_{s}\right)$ or inhibitory $\left(G_{i}\right)$ action on adenylate cyclase, their activation of phospholipase- $C\left(G_{p}\right)$ or activation of ion channels $\left(G_{0}\right)$ (Birnbaumer, 1990a, b). The presence and possible role of the latter $G_{p}$ and $G_{0}$ proteins in small artery smooth muscle, however, is not fully clear. Whether pathways of pharmaco-mechanical coupling in resistance arterial smooth muscle cells are comparable to those found in other tissue remains to be clarified.

Presently it is evident that generation of CAMP (Fig. 1.3) by the activity of the membrane bound adenylate cyclase and the subsequent activation of CAMP dependent protein kinases (protein kinase-A) results in relaxation of smooth muscle cells through effects of cAMP on membrane ion channels (Artalejo et al., 1990; Heesen and De Mey, 1990), calcium extrusion and uptake (Graziano and Gilman, 1987; Kamm and Stull, 1989; Komori and Bolton, 1989) and on the sensitivity of the contractile machinery for calcium (Kamm and Stull, 1989). Accumulation in the vascular smooth muscle cell of cyclic guanosine monophosphate (cGMP) (Fig. 1.3) following stimulation of membrane bound (particulate) or cytosolic (solu- 
CHAPTER 1

ble) guanylate cyclase will also result in a relaxing response in vascular smooth muscle (De Mey et al., 1987; Lincoln, 1989).
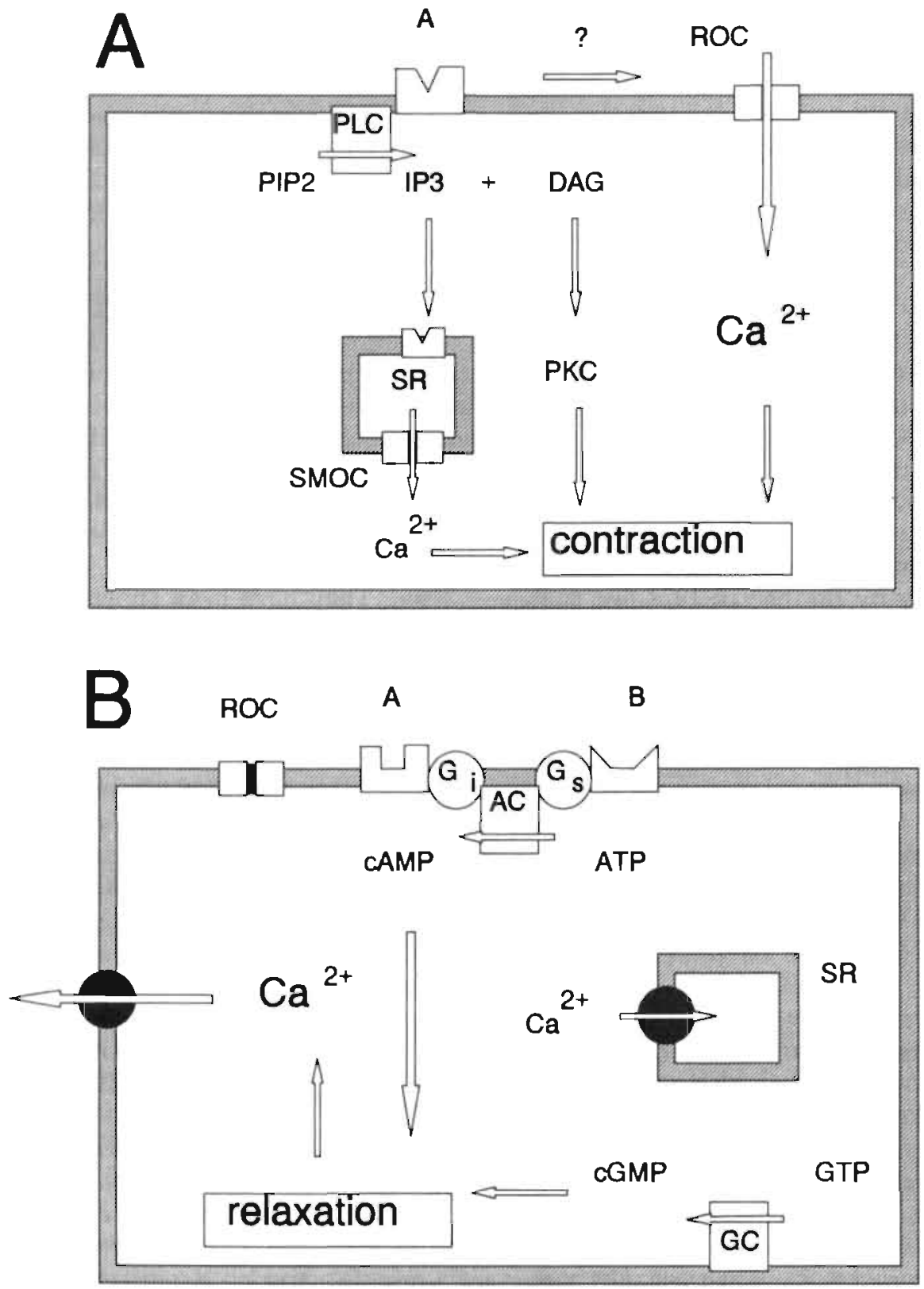
Figure 1.3. Pharmaco-mechanical coupling is achieved through transduction of a stimulus initiated at the cell membrane to the intracellular compartment. One of the activating and one of the inactivating mechanisms of the contractile response in vascular smooth muscle are illustrated.

A) The phosphoinositide pathway: receptor activation (e.g. $\alpha 1$-adrenergic, angiotensin, vasopressin, endothelin, serotonin) leads to the activation of phospholipase C (PLC) that cleaves phosphatidyl biphosphate $\left(\mathrm{PIP}_{2}\right)$ into inositol triphosphate $\left(\mathrm{IP}_{3}\right)$ and diacylglycerol (DAG). Furthermore, receptor activation may open receptor-operated calcium channels (ROC) in the sarcolemma directly or indirectly through second messengers. $\mathbb{I P}_{3}$ induces the release of calcium from the sarcoplasmic reticulum (SR) through second messenger operated calcium channels (SMOC). DAG activates protein kinase C (PKC) which increases the calcium sensitivity of the contractile apparatus and possibly calcium influx.

B) The cyclic nucleotide systems. Receptor stimulation can either inhibit (A) or stimulate $(B)$ adenylate cyclase $(A C)$ through activation of inhibitory $\left(G_{i}\right)$ or stimulatory $\left(G_{5}\right)$ GTP. binding regulatory proteins. This results in reduced or increased production of cyclic adenosine monophosphate (cAMP) which reduces calcium sensitivity of the contractile machinery, stimulates intracellular calcium uptake and calcium extrusion mechanisms and causes hyperpolarisation, which eventually lead to a relaxing response. These are at least partly mediated by activation of protein kinase-A. Furthermore, activation of guanylate cyclase (soluble or particulate) generates cyclic guanosine monophosphate (cGMP). Like CAMP, cGMP exerts vasodilating effects in vascular smooth muscle. The molecular mechanisms are less well understood than those participating in the effects of cAMP.

Some fourty years ago, a third signal transduction pathway was discovered (see: Berridge and Irvine, 1984) that appeared to be a major system through which many vasoactive substances exert a contractile response in vascular smooth muscle; the phosphoinositide pathway (Fig. 1.3). The phospholipid phosphatidyl-inositol 4,5 -biphosphate $\left(\mathrm{PIP}_{2}\right)$ is a minor membrane constituent that can be hydrolised by the membrane bound phospholipase- $\mathrm{C}$ (PLC). This results in the generation of inositol triphosphate $\left(\mathrm{IP}_{3}\right)$ and a diacylglycerol (DAG). The former has been shown to bind to specific receptors on the sarcoplasmic reticulum thereby inducing calcium release from the sarcoplasmic reticulum (Berridge, 1986a, b). IP 3 can be further phosphorylated into inositol tetrakisphosphate $\left(I_{4}\right)$, pentakisphosphate (IP) up to inositol hexakisphosphate $\left(I P_{h}\right)$. From these metabolites $\mathrm{IP}_{4}$ has been suggested to have additional regulatory effects on $\left[\mathrm{Ca}^{2+}\right]_{1}$ (Boynton t' al., 1940). On the other hand diacylglycerol activates protein kinase- $\mathrm{C}$ before being metabolised into arachidonic acid (Berridge, 1986b; Nishizuka, 1986). Protein kinase- $C$ has multiple substrates. It has been suggested to be involved in the phosphorylation of several ion channels and exchangers (Boscoboinik et al., 1990), the phosphorylation of receptors (Huganir and Greengard, 1987; Raymond et al., 1990), decrease of the activity of phospholipase C (Yamatani et al., 1990) and increase of the calcium sensitivity of the contractile apparatus (Adelstein and Sellers, 1987; Khalil et al., 1987; Rasmussen et al., 1987; Drenth et al., 1989). Whether these findings also apply for resistance arterial smooth muscle is unknown. The question whether protein kinase-C is involved in the maintenance of the contractile state of vascular smooth muscle remains therefore under continuous exploration (Forder et al., 1985; Rasmussen et al., 1987; Khalil and Van 


\section{CHAPTER 1}

Breemen, 1988; Sasaguri and Watson, 1989). In following chapters the possible role of protein kinase- $\mathrm{C}$ in the function of vascular smooth muscle cells will be further addressed.

Besides the above mentioned second messenger systems, other systems exist in vascular smooth muscle cells that may also be involved in contractile processes. Different phospholipases are present in the cell which generate second messengers such as phosphatidic acid, and arachidonic acid which in turn serve as substrates for tite lipoxygenase and cyclo-oxygenase pathways (D'Orleans-Juste et al., 1985; Luscher, 1990). Some growth factors have been described to exert contractile responses in isolated large arteries (Berk et al., 1985). Most of the growth factors like platelet derived growth factor (PDGF) or epidermal growth factor (EGF) activate a tyrosine kinase that is incorporated. within the membrane receptor (Blackshear et al., 1988). Activation of this kinase may affect the phosphoinositide system and subsequently contractility of smooth muscle (Boyer et al., 1989).

The different pathways discussed presumably interact resulting in complex feedback mechanisms. Calcium itself for example activates phospolipase-C and calcium dependent potassium channels (Berridge and Galione, 1988) and induces its own release from intracellular stores (Berridge and Galione, 1988; Van Breemen and Saida, 1989).

\section{Mechanotransduction}

Additionally, physical distortion of the cell or cell membrane can activate second messenger systems and ion channels (Kirber et al., 1988; Watson, 1991). Calcium channels have been suggested that are opened by stretch of vascular smooth muscle (Hwa and Bevan, 1986; Laher and Bevan, 1989). Furthermore, stretch has been shown to affect the sensitivity of vascular smooth muscle to pharmacological stimulation (Price et al., 1981; Nilsson and Sjöblom, 1985). Especially in myogenic autoregulatory responses mechanotransduction may play a prominent role. However, concerning the mechanisms of transducing a physical factor into a contractile response, little is known. The excitability of the membrane may be involved (Smeda and Daniel, 1988; Johansson, 1989) in these phenomena as well as second messenger systems (Watson, 1991). Eventually, one has to consider that the vascular smooth muscle cell is attached to an extracellular matrix consisting of structures that may interfere with mechanotransducing mechanisms and cell function. Alterations or malfunctions in this delicately tuned equilibrium of all the controlling mechanisms could lead to pathological situations.

\section{THE VASCULAR WALL AND HYPERTENSION}

At present, besides starvation, infectious diseases and cancer, failure of the cardiovascular system represents a major cause of death for mankind. This dissertation will be confined to fundamental mechanisms of contraction of small artery smooth muscle cells and alterations herein that may possibly be 
involved in the genesis and maintenance of hypertension. Hypertension predisposes for cardiovascular diseases such as angina pectoris, heart failure, sudden death and stroke (Genest et al., 1983). To increase quality and duration of the human lifespan it is therefore of importance to effectively treat hypertension. Effective treatment requires the knowledge of pathological mechanisms involved. Many years of exploration of the development and maintenance of hypertension brought some clarification on potential pathogenic mechanisms. However, full control of these pathologies is not yet achieved and investigation on their mechanisms continues.

Two subtypes of hypertension may be considered. A state of chronically increased blood pressure resulting from a clear cause such as renal insufficiency, hyperaldosteronism, Cushing's syndrome, diabetes or obesity (see: Genest et al., 1983) is termed secondary hypertension. This can frequently be normalised by treatment of the primary cause. In the case of primary or essential hypertension however, the cause for the rise in blood pressure is not fully known. Presently it is believed that different factors may underlie the development of primary hypertension.

\section{Essential hypertension}

One hypothesis that may explain the development of essential or primary hypertension in human or experimental animal models was proposed by Folkow in the late fifties (Folkow et al., 1958). Genetic factors predispose an individual to become hypertensive. Ageing and environmental factors such as diet, mental stress and salt balance increase the probability of these to develop hypertension (Folkow et al., 1958; Folkow, 1982). A pressor substance present in the blood plasma in concentrations that would normally not affect vascular tone would induce a small but sustained vasoconstriction in subjects that have an increased susceptibility for the pressor substance due to altered genetic factors. The global vasoconstriction would in turn increase total peripheral resistance and hence blood pressure. The maintained small but significantly raised blood pressure would lead to a structural adaptation of the resistance vasculature as to unload the active components of the vascular smooth muscle cells and redistribute and normalise wall stress. This results in a thickening of the wall and a narrowing of the lumen of resistance-sized vessels within the arterial tree or in an increase in muscle mass. An increase of the wall to lumen ratio has been suggested to affect the reactivity of the vessel (Folkow, 1986, 1990). The increased reactivity for vasopressor substances consequently leads to a further narrowing of vessel diameter. Finally this vicious circle of positive feedback results in an upward resetting of blood pressure.

This hypothesis was later adapted by Lever (Lever, 1986) in such a way that not only pressor substances were thought to be involved in upregulating vascular structure but also mitogenic factors. These could also be involved in alteration of vascular dimensions, without any initiating change in blood presSure (Overbeck, 1980; Plunkett and Overbeck, 1988; Smeda et al., 1988). It has been repeatedly shown (for review see: Mulvany and Aalkjaer, 1990) that the wall / lumen ratios in 
arteries of hypertensive subjects are increased compared to their normotensive controls. Depending on the vascular bed hypertrophy and hyperplasia of arterial smooth muscle or rearrangement of vascular smooth muscle cells in the medial layer are responsible for the overall structural change (Lee et al., 1983; Plunkett and Overbeck, 1985; Mulvany, 1986; Aalkjaer et al., 1987c; Lee, 1987; Folkow, 1990). In vivo it can be shown that under fully dilated conditions, the minimal resistance to blood flow in hypertensives is significantly increased compared to normotensives, indicating a structural narrowing of the vascular bed (see Folkow, 1990). Furthermore, hyperreactivity of vessels from hypertensive humans and animals has been observed independent of the vasoconstrictor used, which is in agreement with the increased wall / lumen ratio (Lais and Brody, 1978; Mulvany, 1989; Triggle, 1989).

\section{Cellular alterations in hypertension}

Although many differences have been found on the cellular level between vascular smooth muscle from hypertensive and normotensive subjects, no consensus has been reached as to which of the alterations is dominating or would be the primary key factor in the development of the hypertensive state. In fact some observations made in different species or different vascular beds show conflicting results which make the interpretation of factors that are involved in this vascular pathology extremely difficult. Also in this aspect, multifactorial interactions take place which, provided they occur under the right circumstances, will amplify or synergise with each other.

Vascular smooth muscle cells of spontaneously hypertensive rats show in many cases a hypersensitivity for contractile substances (Lais and Brody, 1978; Webb, 1984; Triggle, 1989; Kong et al., 1991), whereas in essentially hypertensive humans the opposite has been found (Aalkjaer and Mulvany, 1983; Aalkjaer et al., 1987b). Hypersensitivity to vasoconstrictors, however has been suggested to arise either from an altered affinity of receptors on the smooth muscle cell membrane (see Michel et al, 1989, 1990), an increased or decreased number of available receptors or an alteration in the transducing mechanism of the particular receptor. Uehara et al., (1988) and Koutouzov et al., (1987) for example, have shown that the activity of phospholipase-C is increased in spontaneously hypertensive rats compared to their genetically related but normotensive Wistar Kyoto rats (WKY). Upstream in the phosphoinositide pathway it has been observed that the function of specific G-proteins may be altered in hypertension (Asano et al., 1988; Johnson et al., 1991). Concerning receptor number and properties it has been suggested that adrenoceptor characteristics are different in hypertensive subjects. Whether these are due to altered sympathetic activity or changes in different mechanisms has to be established (Abel and Hermsmeyer, 1981; Abboud, 1982; Head, 1989; de Champlain, 1990; Esler et al., 1990;Michel et al., 1990a).

Calcium has been implicated as being responsible for changes in vessel reactivity (Sharma and Bhalla, 1988; Bukoski, 1990). It has been shown in spontaneously hypertensive rats but not in human vessels that the sensitivity for calcium in the presence of agonists is increased (Mulvany and Nyborg, 1980; Aqel et al., 1986, 1987; 
Rusch and Hermsmeyer, 1988; Boonen and De Mey, 1990). This has been attributed to either alterations in the number or kinetics of the calcium channel itself, in modifications of its controlling mechanisms or in an alteration of the calcium sensitivity of the contractile apparatus. Evidence presently favours the hypothesis that it is a membrane defect which may be the primary cause for some forms of hypertension (Harder and Hermsmeyer, 1983; Hermsmeyer and Harder, 1986; Exton, 1988; Sharma and Bhalla, 1988; Swales, 1990). Harder and Hermsmeyer, (1983) suggested that defects in smooth muscle cell membrane ion handling would result in altered calcium homeostasis and consequently changes in cell function. It is still unknown whether the described alterations in cellular systems are epiphenomena that occur as a result of alterations in control systems during the hypertensive state or whether these contribute significantly to the development or maintenance of high blood pressure. This aspect is of exceptional importance in the understanding of hypertension.

\section{Alterations in vascular control mechanisms}

As pointed out above, changes on the cellular level may evolve from alterations on a higher level. For example, a change in the number or affinity of adrenergic receptors (Michel et al., 1989, 1990) may be related to the observed changes of the sympathetic nervous system in hypertensives (Aprigliano and Hermsmeyer, 1977; Abboud, 1982; de Champlain, 1990; Esler et al., 1990; Mark, 1991). Effects of the sympathetic nervous system on vascular function have been studied in a wide variety of species and vascular beds (Abboud, 1982; Aalkjaer et al., 1987a). The endothelial layer which is, as discussed above, an important regulator of vascular function, has been shown to be altered in many aspects during different forms of hypertension. Endothelium-dependent relaxation of vascular smooth muscle is impaired in vessels from hypertensives (Winquist et al., 1984; De Mey and Gray, 1985; Lüscher, 1990a, b), which may be due to a reduced release of EDRF or increased production of EDCF. Furthermore, alterations in the renin angiotensin system have been implicated in the development of hypertension (Lüscher, 1990b; Scott-Burden et al., 1992). Angiotensin Il not only induces a contractile response in vascular smooth muscle (Griendling et al., 1987, 1989), it also has trophic influences on vascular smooth muscle (Berk et al., 1989; Daemen et al., 1991; Schelling et al., 1991).

Many factors appear to be altered in hypertension. Yet, it remains unknown whether some actually contribute to the development of the increased blood pressure or result from it. Especially on the level of the resistance vasculature, it is unresolved whether cellular processes may or may not be involved in the emergence of the hypertensive disease. 


\section{THIS THESIS}

The objective of the present thesis was to investigate mechanisms of excitation-contraction coupling in small arterial smooth muscle and to evaluate whether changes in vascular reactivity on a resistance arterial level are involved in the aetiology and maintenance of hypertension.

Excitation-contraction coupling mechanisms were studied in multicellular resistance arterial preparations. Effects of calcium antagonists were compared in large and small arteries paying particular attention to differences in electromechanical and pharmacomechanical coupling and in receptor characteristics (Chapter 3). The role of calcium was assessed during pharmacological and electromechanical stimulation of small arteries and effects of a dihydropyridine calcium antagonist and agonist were related to these (Chapter 4). Mechanisms that are involved in the transduction of a pharmacological stimulus into the release of intracellular calcium, calcium influx and subsequent contraction of small arteries were evaluated with respect to the involvement of GTP-binding regulatory proteins (Chapter 5). The role of protein kinase- $C$ in different mechanisms of excitation-contraction coupling in small arteries was evaluated (Chapter 6). Lastly, the importance of mechanotransducing pathways in contraction of small arteries and the possible mechanisms of this transduction pathway were assessed during static distension of small arteries (Chapters 7 and 8). From the results obtained a model of excitation-contraction coupling mechanisms in small arteries will be proposed which may explain hypersensitivity and hyperreactivity in small arteries of adult spontaneously hypertensive rats.

Whether resistance arterial changes in reactivity may be responsible for development or maintenance of hypertension was assessed using in vitro and in vivo approaches (Chapters 9 and 10). Finally, an integration was made regarding mechanisms of excitation-contraction coupling in small arteries, their involvement in changes in vascular reactivity and their relation to animal models of hypertension (Chapter 11). 


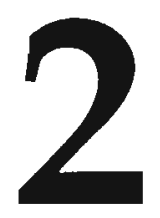

\section{Material and Methods}




\section{Experimental animals}

For most experiments, male normotensive Wistar Kyoto rats (WKY) and age matched spontaneously hypertensive rats (SHR) of the Okamoto strain were used. The age of the rats ranged from 6 to 20 weeks depending on the experimental objective. Rats were obtained from locally inbred strains (University of Limburg, Maastricht, The Netherlands). Rats were housed in standard laboratory cages. They received food (type RMM-TM, Hope Farms, Woerden, The Netherlands) and water ad libitum. Prior to the isolation of small arteries, rats were sacrificed by a sharp blow on the head and exsanguination.

\section{Measurement of mechanical properties of small arteries}

\section{The myograph}

In the introductory chapter the importance of small arteries in the control of total peripheral resistance and their role in hypertension has been emphasised. Measurement of mechanical, structural and pharmacological properties of specific vascular segments was in the past limited mainly to large vessels because of technical difficulties. Some 15 years ago, however, a technique for measuring isometric force development in small arteries was developed into a working tool for the use of small arteries $(100-400 \mu \mathrm{m}$ in diameter) by Mulvany and Halpern, (1977) (Fig. 2.1). Ring segments of small vessels can be mounted in an organ bath on two stainless steel wires between a displacement device and an isometric force transducer (JP Trading Corp., Risskov, Denmark). This technique enables the study of mechanical and pharmacological properties of small arteries or veins of different origin and is used for this purpose throughout this study.

\section{lsolation and mounting of arteries}

The mesentery was isolated from the rats, rinsed with a buffered physiological salt solution and pinned out on a petri dish coated with Silgard (Dow Corning Corp.) filled with a buffered physiological salt solution. Third to fourth order side branches of the superior mesenteric artery were dissected from the mesentery. In some comparative studies, segments of the superior mesenteric artery were also dissected directly after branching of this artery from the abdominal aorta. A stainless steel wire with a diameter of $40 \mu \mathrm{m}$ was inserted into the lumen of the vessel proximal from the "test" segment before it was taken out of the mesentery. To exclude influences of sympathetic nerveendings on mechanical and pharmacological properties (Vanhoutte et al., 1981), vessels were chemically sympathectomised, except when specifically indicated. This was achieved by an incubation of the vessels during 10 minutes at $37^{\circ} \mathrm{C}$ in a modified Krebs solution ( $\mathrm{pH} 4.0$ ) that contained $300 \mu \mathrm{g} / \mathrm{ml}$ 6-hydroxydopamine (Aprigliano and Hermsmeyer, 1977). Sympathectomy was considered successful when contractile responses induced by high potassium were similar in the absence or presence of the $\alpha$-blocking agent phentolamine. A second wire was then inserted into the lumen of the vessel and the vessel segments were then mounted for recording of isometric force development (Fig 2.2). 


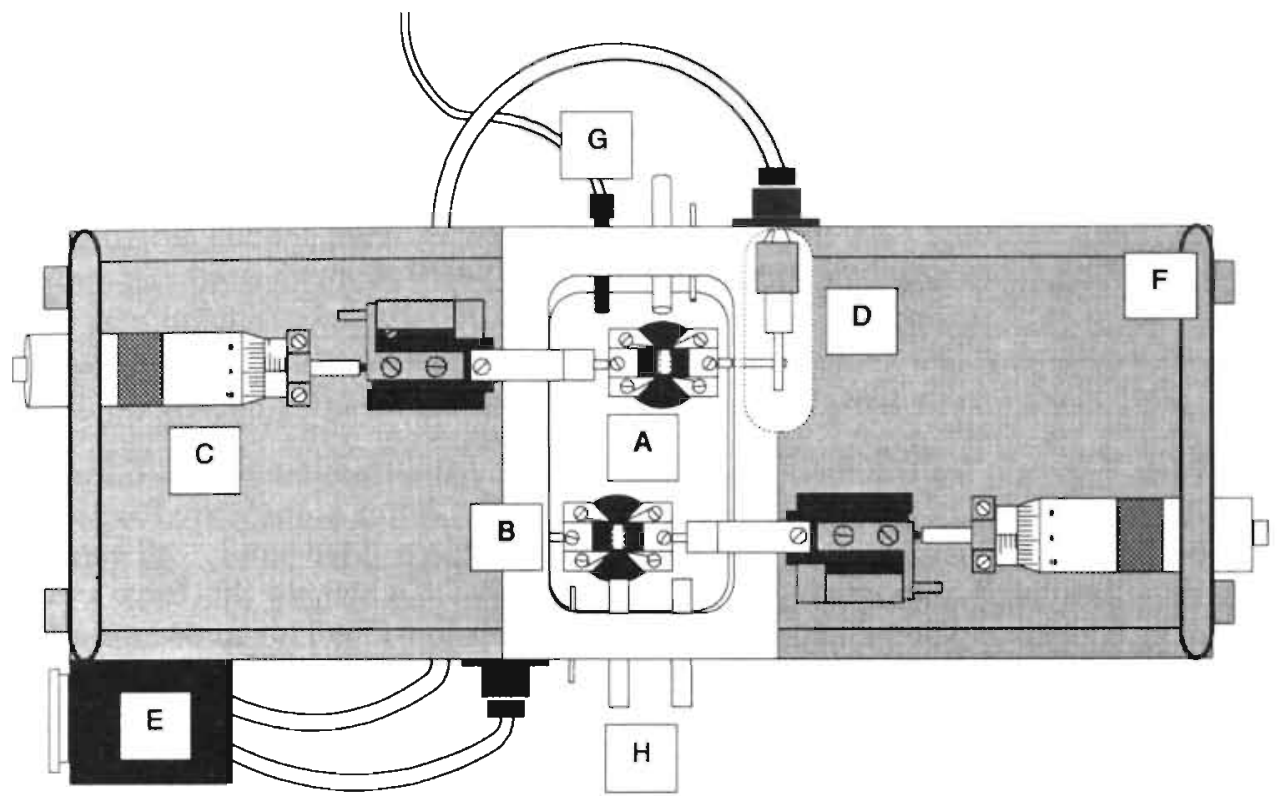

Figure 2.1 Drawing of the small vessel myograph (sce text) as it was used throughout the experiments that are described in the present thesis. The following essential parts can be distinguished: A stainless steel organ bath with a volume of $10 \mathrm{ml}$ (A) in which in parallel two preparations can be studied. Two cover glasses in the bottom enable visualisation of the preparations from below. Two pair of stainless steel supports (B) of which one is connected to a displacement device (C) and the other to a force transducer (D) that is embedded in the wall of the organ bath. The transducers can be connected (E) with an amplifier and chart recorder. The myograph is waterheated through an internal circuit $(F)$ of canals connected to an external waterbath. Actual temperature of the solution in the bath is registered through a temperature probe $(G)$ that is connected to the amplifier. Solutions in the bath can be aerated, removed or added through various small tubes $(\mathrm{H})$ that enter the bath from both sides.

\section{Mechanical parameters}

The mechanical properties of small arteries can be described by a model that was previously described by Hill (see: Mulvany and Aalkjaer, 1990). This model consists of a viscous element which is coupled in parallel and in series to elastic elements that are mainly comprised of extracellular matrix components. Passive and active isometric force can be picked up by the force transducer in the myograph. From these some of the mechanical properties of the vessel can be deduced.

In the myograph the vessel is distended to an ellipsoid conformation (Fig 2.2). An internal lumen diameter $(D,(\mu \mathrm{m})$ ) can be calculated which the vessel would have in an imaginary circular situation. Diameter is calculated 
from the thickness of the wires $\left(\mathrm{d}_{\mathrm{w}}\right)$ and the imposed displacement of the micrometer $\left(\mathrm{X}_{\mathrm{m}}\right)$.

$$
D=\frac{2 X_{m}+\left(2 d_{w}+\pi d_{w}\right)}{\pi}
$$

Wall tension ( $W T$, expressed in $\mathrm{mN} / \mathrm{mm}$ ) is calculated as:

$$
W T=\frac{F}{28}
$$

in which $\mathrm{F}$ stands for the force $(\mathrm{mN})$ measured by the transducer and $\delta$ for the longitudinal length of the vascular preparation. The latter is measured with the use of a microscope equipped with a scaled eye piece. Measured wall tension can be subdivided into resting wall tension (RWT), which is the force developed in a resting distended vessel, total wall tension (TWT), representing the force developed upon stimulation of the vessel with a vasoactive substance, and active wall tension (AWT = TWT-RWT). With the use of Laplace's equation an effective transmural pressure (ETP, in $\mathrm{mN} / \mathrm{mm}^{2}$ ) can be calculated which is an estimate of the transmural pressure required to stretch the vessel to the combination of WT and diameter of interest.

$$
E T P=\frac{W T}{D / 2}
$$

Finally, wall stress (WS, in $\mathrm{mN} / \mathrm{mm}^{2}$ ) normalises wall tension for media thickness $\left(M_{t}\right.$, see below).

$$
W S=\frac{W T}{M_{i}}
$$

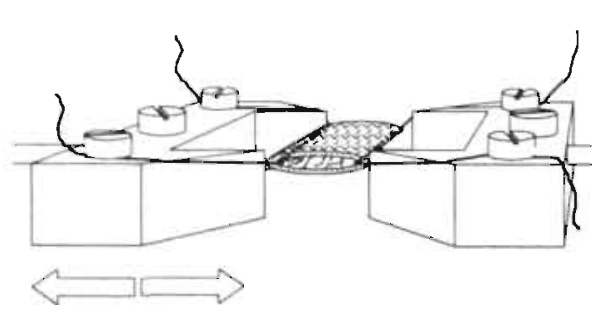

Figure 2.2 Illustration of a small artery segment mounted on two wires between two stainless steel supports that are either connected to a force transducer or a displacement device with which one is able to distend the segment in the indicated directions (arrows). The vessel segment has a length and diameter of about $2 \mathrm{~mm}$ and 200 $\mu \mathrm{m}$ (see Table 2.1 ), respectively.

\section{Standardisation}

The study of mechanical properties and pharmacological behaviour of small arteries from different species or different rat strains requires that experiments are performed under near physiological circumstances. This means 
that vessel reactivity is studied at $37^{\circ} \mathrm{C}$, in buffered salt solution with sufficient concentrations of $\mathrm{O}_{2}$ and glucose for maintenance of basal function. Furthermore, to draw conclusions or to make comparisons of results obtained in vessels of different origin or from different species it is essential to start with similar standardised conditions. It is not only of importance to mimic external physiological factors such as temperature or $\mathrm{pH}$ but also vascular qualities as they would have been in vivo. Muscle behaviour depends very much on the initial length or stretch that is applied (Mulvany and Warshaw, 14, i); Moreland et al., 1988; Barany et al., 1990; Rembold and Murphy, 1990). The reactivity to vasuactive drugs is also affected by physical parameters (Price et al., 1981; Nilsson and sjöblom, 1985).

Therefore, before experimentation, the vessel lumen diameter has to be normalised using well defined criteria. Two approaches can be mentioned in this respect. The internal lumen diameter of the vessels can be set at $90 \%$ of the diameter the vessel would have in vivo when subjected to a transmural pressure of $100 \mathrm{~mm} \mathrm{Hg}$. It has been shown previously (Mulvany and Halpern, 1977; Mulvany and Warshaw, 1979) that at this diameter, mesenteric resistance sized arteries of adult normotensive rats develop their maximal force. Another approach is to find the diameter at which an individual vessel contracts maximally to a direct stimulation of the vascular smooth muscle (De Mey and Brutsaert, 1984).

Advantages of the first approach are that the determination of the internal lumen diameter can be obtained very accurately from the passive characteristics of the vessel by finding the intersection of the isobaric line for $100 \mathrm{~mm}$ $\mathrm{Hg}$ (obtained using Laplace's equation) and the passive length-tension relation (Fig. 2.3), which can be fitted by an exponential curve as follows:

$$
R W T=A \cdot e^{\beta D}
$$

in which $A$ is a constant, $\beta$ the stiffness factor and $D$ the diameter of the vessel. Therefore, the diameter corresponding with the intersection of these two lines is physically and mathematically strictly defined and standardised. Disadvantages are that the active component of the vessel wall is not included in the determination of the lumen diameter and that secondly, the in vivo transmural pressure at the site of the vessel segment is not necessarily $100 \mathrm{~mm} \mathrm{Hg}$. This implies that in arteries or veins of other vascular beds or other species, the standardised lumen diameter may be an over- or an underestimation, resulting in a distension of the vessel that is below or above the "physiological" value. As will become clear in following chapters (chapters 7 and 8), stretching of vessels has significant effects on sensitivities for pharmacological stimuli. This has serious implications for comparison of results obtained in vessels of normotensive and hypertensive rats and for their interpretation.

The performance of the active component is essential in the determination of the optimal lumen diameter in the latter approach. Figure 2.3 shows a typical example of the protocol used for the determination of the optimal lumen diameter $\left(D_{0}\right)$. Under fully relaxed conditions the vessel diameter is increased stepwise and is intermittently stimulated with a depolarising high 


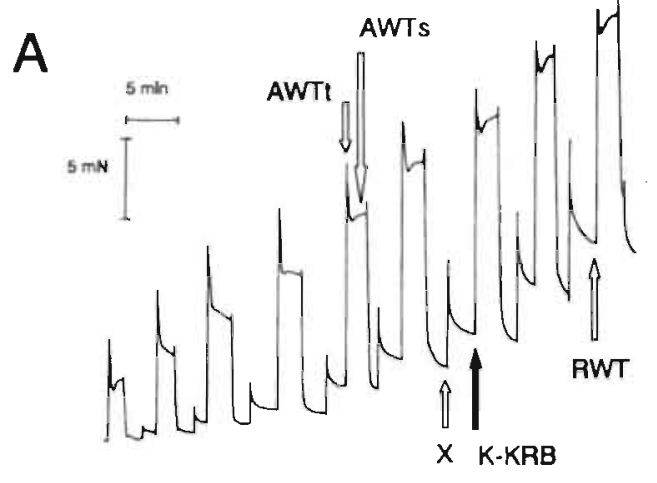

Figure 2.3 A) Typical tracing illustrating the protocol for the determination of the optimal internal lumen diameter $\left(D_{0}\right)$. Sympathectomised vessels mounted in a myograph are stretched $(X)$ and intermittently stimulated with $125 \mathrm{mM} \mathrm{K}-\mathrm{KRB}$. This procedure is repeated until the transient active response to $125 \mathrm{mM} \mathrm{K-KRB} \mathrm{(AWT)}$ has reached a maximum. From this protocol a diameter - wall tension relation can be plotted as shown in B)

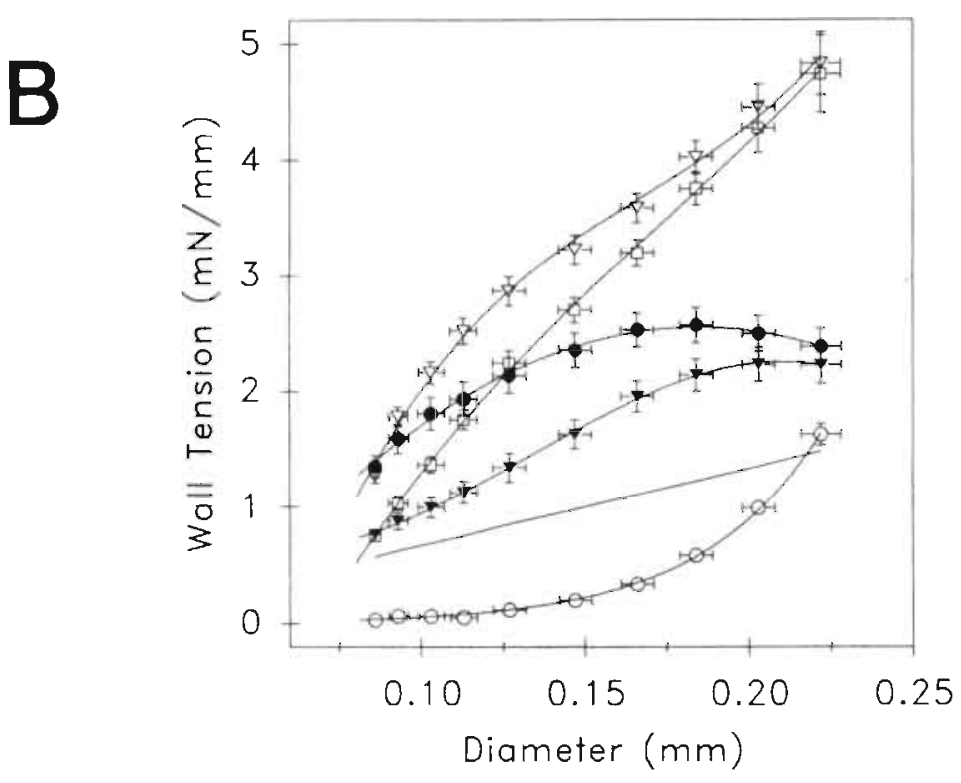

B) Diameter tensiun relationship in sympathectomised mesenteric resistance sized arteries. Figure shows resting wall tension (RWT, open circles), total transient (TWT, and sustained (TWT) wall tension (open triangle and square, respectively) and transient $\left(A W T_{1}\right.$ ) and susl.ined (AWT) active wall tension (closed circles and triangles, respectively), which are obtained by subtracting RWT from TWT. Values are means \pm SEM with $n=31$. The straight line represents the isobaric line at which ERTI' would be $100 \mathrm{~mm}$ $\mathrm{Hg}$. Finally the diameter of the preparations is set at the diameter where AWT, is maximal. 
potassium solution $\left(\left[\mathrm{K}^{+}\right], 125 \mathrm{mM}\right)$. In sympathectomised arteries this will result in a biphasic response consisting of an initial transient ( $A W_{1}$ ) and a subsequent sustained contraction $\left(\mathrm{AWT}_{\mathrm{s}}\right)$ (Fig 2.3). Plotting the resting and transient active wall tension against the diameter will give a resting and active lengthtension curve (Fig 2.3). As mentioned before, the resting tension versus diameter curve describes an exponential relation. The circumferential length-active tension curve can be fitted with a polynomial equation. The top of the curve is observed at $D_{0}$. This means that regardless the true transmural pressure that may be present in vivo a normalised lumen diameter can be found at which the vessel performs maximally. Some evidence shows that in vivo, vessels preferentially "live" at the diameter at which they can respond maximally to stimulation (Lash et al., 1991). These observations strengthen our decision to use the determination of the "optimal lumen diameter" being the diameter at which the vessels respond maximally to a direct depolarising stimulus. However, also this approach has some disadvantages. The top of the active wall tension diameter relation is quite flat (Fig. 2.3 ) which means that a similar maximum response extends over a relatively broad range of diameters and resting wall tensions. Furthermore, the choice of the stimulus to obtain $D_{0}$ could be of importance. Different stimuli may give different $D_{0}$. Maximal stimulation of the preparation with a mixture of known vasoconstrictors could be an obvious solution for this problem. However, some vasoconstrictors act as vasodilators in vessels from different sources. This complicates the choice of vasoconstrictors that may be used to maximally stimulate a vessel segment. Therefore, to preserve a standardised approach, we used a receptor independent direct stimulation of the contractile apparatus by a depolarising high potassium solution. Table 2.1 compares $D_{0}$ and the corresponding maximal active wall tension and effective transmural pressures as they were obtained in vessels from WKY and SHR with the different ways of standardisation.

\section{Pharmacological evaluation of resistance arterial reactivity}

Vascular reactivity and mechanisms of excitation-contraction coupling responsible for the contractile function of small arteries were assessed using pharmacological tools. This approach was used for the following reasons: i) measurement of contractile function requires intact preparations, ii) the small size of these vessels hinders biochemical evaluation of processes that take place on a molecular level, iii) some biochemical events in the excitation-contraction coupling process depend on an intact integrity of the vessel wall (Watson, 1991) and can therefore not be assessed in homogenates of smooth muscle cells.

The pharmacological tools that were used included noradrenaline (and phenylephrine), angiotensin II, arginine vasopressin, serotonin and endothelin. These substances have been shown to significantly affect vascular function in vivo. They are derived from systems that exert major regulatory roles on vascular reactivity, such as the sympathetic nervous system, the renin-angiotensinaldosterone system, the endothelium and blood borne substances involved in inflammatory responses (D'Orleans-Juste et al., 1985; Furchgott and Vanhoutte, 1989; Griend- 


\section{CHAPTER 2}

ling, 1989; Vanhoutte, 1989; Burnstock, 1990; Raymond, 1990; Miller and Vanhoutte, 1991). Furthermore, these may be involved in the development and maintenance of hypertension (Abboud, 1982; Genest, 1983; Lever, 1986; Head, 1989; de Champlain, 1990; Kishi, 1990).

Table 2.1. Mechanical properties of isolated sympathectomised mesenteric resistance-sized arteries from Wistar Kyoto and spontaneously hypertensive rats.

\begin{tabular}{|c|c|c|c|c|}
\hline & \multicolumn{2}{|l|}{ WKY } & \multicolumn{2}{|l|}{ SHR } \\
\hline & I & II & 1 & II \\
\hline$D$ & $184 \pm 5$ & $199 \pm 6$ & $192 \pm 7$ & $194 \pm 8$ \\
\hline RWT & $0.58 \pm 0.04$ & $0.61 \pm 0.02$ & $0.85 \pm 0.05^{a b}$ & $0.63 \pm 0.01$ \\
\hline ERTP & $6.33 \pm 0.43$ & $6.28 \pm 0.28$ & $8.78 \pm 0.35^{\mathrm{ab}}$ & $6.79 \pm 0.31$ \\
\hline$(\mathrm{mm} \mathrm{Hg})$ & $84.1 \pm 5.7$ & $83.5 \pm 3.7$ & $116.8 \pm 4.6^{\text {ath }}$ & $90.3 \pm 4.1$ \\
\hline$A W T_{1}$ & \multicolumn{2}{|l|}{$2.57 \pm 0.15$} & \multicolumn{2}{|l|}{$3.13 \pm 0.19^{a}$} \\
\hline$A W T_{\text {, }}$ & \multicolumn{2}{|l|}{$2.14 \pm 0.14$} & \multicolumn{2}{|l|}{$3.00 \pm 0.20^{a}$} \\
\hline$\beta$ & \multicolumn{2}{|c|}{$22.3 \pm 1.4$} & \multicolumn{2}{|c|}{$23.1 \pm 1.4$} \\
\hline$n$ & 31 & 31 & 32 & 32 \\
\hline
\end{tabular}

Comparison of different standardisation techniques with respect to mechanical vessel properties in mesenteric resistance arteries of 20 week old $\sigma^{x}$ WKY and SHR rats. Values in group I were obtained when D equals the diameter at which potassium induced contractions were maximal whereas group II represents values when D is chosen as $90 \%$ of the diameter at which the effective pressure would be $100 \mathrm{~mm} \mathrm{Hg}$. D ; diameter $(\mu \mathrm{m})$ $\mathrm{RWT}$; resting wall tension $(\mathrm{mN} / \mathrm{mm})$. ERTP; effective resting transmural pressure (in $\mathrm{mN} / \mathrm{mm}^{2}$ and $\mathrm{mm} \mathrm{Hg}$ ). AWT, and AWT, ; maximal transient and sustained response to maximal depolarisation, respectively $(\mathrm{mN} / \mathrm{mm}) . \beta\left(\mathrm{mm}^{-1}\right)$ represents a stiffness factor which is a measure of the rigidity of the vessel. $\mathrm{n}$; number of observations. Values are means \pm SEM. "and ${ }^{b}$ indicate statistical significance of difference between WKY and SHR and group I and II, respectively (ANOVA followed by t-test, $P<0.05$ ).

Vessel reactivity was evaluated by construction of concentration response curves to various vasoactive substances. Sensitivities and maximal force development for these substances could be derived from these. Concentrations of vasoactive substances needed to half maximally induce or inhibit a contractile response $\left(\mathrm{EC}_{50}, \mathrm{IC}_{50}\right.$, respectively) in isolated arteries were determined from the concentration response curves using a least square sigmoidal curve fit (Graphpad Software, San Diego, CA, USA). Concentration response curves for agonists were produced by cumulatively increasing the concentration of the agonist. Concentration response curves for the contractile effect of extracellular calcium $\left(\left[\mathrm{Ca}^{2+}\right]_{\mathrm{e}}\right)$ were performed as follows: firstly, depletion of intracellular 
calcium stores by stimulating the vessels twice in $\mathrm{Ca}^{2+}$-free $\mathrm{KRB}$ with $10 \mu \mathrm{M}$ noradrenaline. Thereafter, in the presence of a stimulus, calcium was readministered to the bath to increase $\left[\mathrm{Ca}^{2+}\right]_{e}$ in a cumulative manner.

\section{Measurement of $\left[\mathrm{Ca}^{2+}\right]_{i}$ in mesenteric resistance sized arteries}

Measurements of intracellular free calcium concentration $\left(\left[\mathrm{Ca}^{2+}\right]_{i}\right)$ were performed simultaneously with force in mounted mesenteric resistance sized arteries using the fluorescent calcium indicator dye Fura-2. The method used for measuring $\left[\mathrm{Ca}^{2+}\right]_{i}$ in these arteries has been described in detail by Jensen et al. (1992). The principle of this technique has been reviewed elsewhere (Grynkiewicz et al., 1985). Briefly, the technique consists of loading of the cells in the preparation with the calcium sensitive fluorescent dye Fura-2. The Fura-2 AM ester passes the cell membrane freely and is in the cell cleaved into Fura-2 and the ester by esterases, thereby capturing the membrane impermeable Fura- 2 in the cell. Depending on whether calcium is bound to the Fura-2 molecule or not, the molecule will emit light when excited with light having a wavelength of 340 and $380 \mathrm{~nm}$, respectively. Taking the ratio of the emitted signal at both wavelengths gives a relative value for $\left[\mathrm{Ca}^{2+}\right]_{i}$ that is independent of the intensity of the emitted light or the thickness of the preparation.

Figure 2.4 shows a schematic drawing of the setup for measuring $\left[\mathrm{Ca}^{2+}\right]_{i}$ in small arteries. Preparations were mounted in a single channel myograph and loaded for 4 hours at room temperature in a KRB solution that contained 10 $\mu \mathrm{M}$ Fura-2-AM, 0.1\% cremophor EL, 0.5\% dimethyl sulfoxamine (DMSO) and $0.02 \%$ pluronic F127. The myograph was placed on an inverted microscope (Zeiss, Axiovert 10). The emission of light, caused by excitation of Fura-2 at 340 $\pm 5 \mathrm{~nm}$ and $380 \pm 5 \mathrm{~nm}$ (delivered by a $100 \mathrm{~W}$ Xenon lamp), was measured from the focal area (media) of the preparation through a $500-530 \mathrm{~nm}$ filter using a photomultiplier. The emission signals and force were measured with 10 second intervals and stored on an IBM personal computer during experimentation. Emission signals were calibrated using the following equation;

$$
\left[\mathrm{Ca}^{2+}\right]_{i}=K_{d} \beta \frac{\left(R_{\max }-R\right)}{\left(R-R_{\min }\right)}
$$

The dissociation constant $\left(K_{d}\right)$ for Fura- 2 at $37^{\circ} \mathrm{C}$ was taken as $224 \mathrm{nM}$ (Grynkiewicz et al., 1985; jensen et al., 1992). $\beta$ represents the ratio between background subtracted emission signals at $380 \mathrm{~nm}$ excitation under calcium free and calcium saturated conditions. Minimal ratio $\left(R_{\min }\right)$ was measured in calcium free HEPES buffered KRB with addition of $15 \mu \mathrm{M}$ ionomycin. Maximal ratio $\left(\mathrm{R}_{\max }\right)$ was subsequently measured in HEPES buffered $\mathrm{KRB}$ containing $5 \mathrm{mM}$ calcium. Following measurements of the $340 \mathrm{~nm}$ and $380 \mathrm{~nm}$ emission signals for the calculation of $R_{\min }$ and $R_{\max }$, the $\mathrm{Ca}^{2+}$-insensitive emission signals were measured by quenching the calcium sensitive Fura-2 remaining in the vessel with 8 $\mathrm{mM} \mathrm{Mn} \mathrm{Mn}^{2+}$. Background emissions were used for background subtractions of intensities measured for the 340 and $380 \mathrm{~nm}$ wavelengths. 


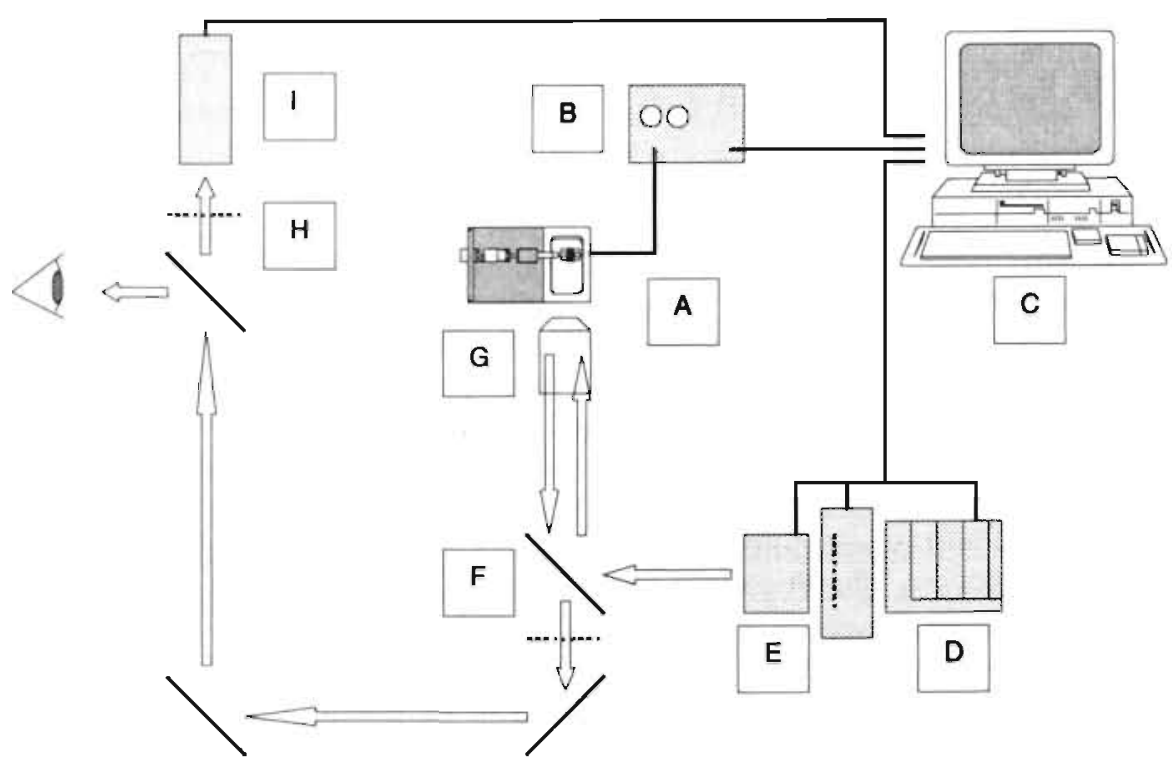

Figure 2.4 Schematic drawing of the setup used for simultaneous measurement of $\left[\mathrm{Ca}^{2+}\right]$, and force in mesenteric small arteries that are mounted in a single channel myograph (A) that is placed on a Zeiss invertoscoop. Force is amplified (B) and recorded on an IBM personal computer (C). The computer controls the intensity of the Xenon light source (D) the filter changer and the shutter $(E)$. Light is reflected by a dichroic mirror $(F)$ through a Zeiss LD Acroplan long working distance objective $(G)$ to the preparation. Returning emitted light passes the dichroic mirror and a band filter $(500-530 \mathrm{~nm})(\mathrm{F})$. Before entering the photomultiplier (I) light passes a second band pass filter $(<700 \mathrm{~nm})(\mathrm{H})$. The photomultiplier signal is stored on the computer.

\section{Measurement of membrane potential}

Evaluation of electrophysiological properties of the vascular smooth muscle cells in the mesenteric artery preparations, simultaneously with measurements of force development, were performed with the use of glass microelectrodes. Figure 2.5 shows a simplified diagram of the setup that was used to measure membrane potential $\left(\mathrm{V}_{\mathrm{n}}\right)$.

The technique was previously described (Mulvany et al., 1982). Vessels were mounted in a single channel myograph to record isometric force development as was described above. The myograph was placed on a vibration-damped stage. Single vascular smooth cells were impaled with glass microelectrodes ( $R$ $\approx 40-90 \mathrm{M} \Omega$ ) filled with $3 \mathrm{M} \mathrm{KCl}$ with the aid of the oscilloscope reading and by gentle tapping on the microelectrode holder (Fig 2.5). Recordings of membrane potential had to fulfill the following criteria: impalements should i) last 
longer than 2 minutes, ii) stabilise rapidly and iii) after withdrawal of the electrode, the baseline should return to the pre-impalement level i.e., electroderesistance should remain constant. Preheated and aerated $\left(95 \% \mathrm{O}_{2}\right.$ and $5 \% \mathrm{CO}_{2}$ ) solutions were perfused continuously through the organ bath.

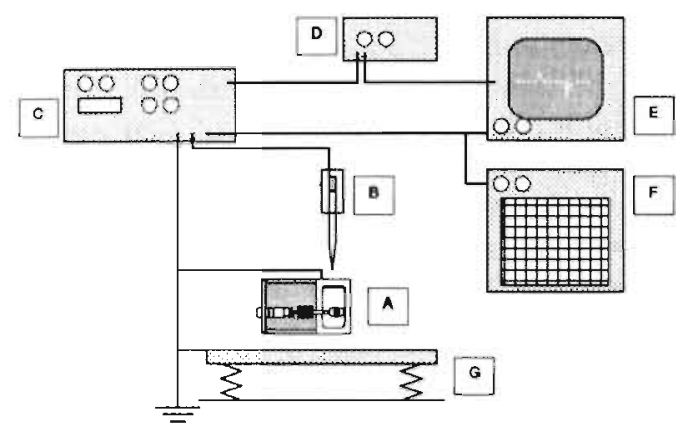

\section{Tissue culture of arteries}

Figure 2.5 Setup for measuring membrane potential in smooth muscle cells of resistance arteries mounted in a myograph (A). Glass microelectrodes filled with $3 \mathrm{M} \mathrm{KCl}$ (B) are fixed in a microelectrode holder (Ag-AgCl cell encapsulated in a plexiglass holder) and connected to a microelectrode amplifier (C) (WPI Inc., model 707). The current pulse through the electrode that is delivered by a pulse generator (D), is balanced by adjustment of the microelectrode amplifier and visualised on the oscilloscope (E) and pen recorder $(\mathrm{F})$. The myograph and microelectrode holder are stabilised by placement on a vibration-damped surface $(G)$.

\section{rationale}

The question whether alterations in sensitivity for vasoconstrictors are a consequence or the cause of the altered haemodynamics in hypertension is still unresolved. Furthermore, changes in excitation-contraction coupling may result from altered concentrations of circulating or local vasoactive substances (Webb, 1984; Burnstock, 1990; Esler et al., 1990; Luscher, 1990; Scott-Burden et al., 1992). To separate specific parameters, that could be involved in this respect, without affecting the total of interacting events in vivo, is extremely difficult. Cell culture offers some advantages in this respect. One is able to control very precisely the conditions of the culture period. But it has been shown that characteristics of isolated vascular smooth muscle cells change in culture from a contractile into a synthetic phenotype (Chamley-Campbell and Campbell, 1981; Chamley-Campbell et al., 1981). Therefore, the study of effects of vasoactive substances or mechanical factors on excitation contraction coupling in isolated single vascular smooth muscle cells could give an erroneous view of their function in a multicellular microenvironment (Stadler et al., 1989). To exclude influences of vasoactive substances and effects of pressure and flow on sensitivities for vasoconstrictors in small arteries on the one hand and maintain microenvironmental conditions and cellular phenotype on the other hand, advantages of both in vivo approaches and cell culture were combined. Mechanical and pharmacological properties of isolated 
mesenteric arteries of WKY and SHR were recorded following short term ( 4 days) tissue culture.

\section{Tissue culture}

The technique for tissue culture of mesenteric arteries was previously described (De Mey et al., 1989). Vessels were isolated from WKY and SHR rats, sympathectomised (Boonen et al., 1991) and suspended without distension in culture dishes filled with $2.5 \mathrm{ml}$ Dulbecco's minimal Eagle's modified medium (GIBCO) on a non-distending stainless steel wire. The medium was supplemented with $20 \%$ fetal calf whole blood serum. The fetal calf serum was dialysed to remove low molecular weight contractile factors and polyamines (Boonen et al., 1991). Vessels were incubated for $1-4$ days at $37^{\circ} \mathrm{C}$ in an atmosphere of $95 \% \mathrm{O}_{2}$ and $5 \% \mathrm{CO}_{2}$ at $97 \%$ humidity after which the vessels were mounted in a myograph for evaluation of pharmacological and mechanical properties.

\section{Structural parameters of small arteries}

Following short term treatment of WKY rats in vivo with vasoconstrictors (Chapter 10), structural dimensions of small mesenteric arteries were evaluated using a software package for personal computer (Jandel Scientific Video Analysis Software, (JAVA V 1.21), Jandel Scientific, Corte Madera, CA, USA). Vessels were isolated from treated rats for evaluation of pharmacological and mechanical characteristics after which they were fixed at $D_{0}$ for 30 minutes at $37^{\circ} \mathrm{C}$ in phosphate buffered ( $\mathrm{pH} 7.4$ ) formaldehyde $(4 \%)$. Preparations were paraffin embedded and cross sectioned using standard histological techniques. Cross sections $(4 \mu \mathrm{m})$ were stained for elastin using Lawson's solution (Boom B.V., Meppel, The Netherlands).

Video images were generated from the cross sections using a Zeiss Axioscope (Zeiss, Germany) and a standard CCD camera (Stemmer, Germany). The following structural parameters were measured; internal and external medial circumference, which were respectively demarcated by the internal and external elastic lamina. From these, assuming circular vessel conformation, the lumen area and the total area could be derived. Medial cross sectional area (CSA) was obtained by subtracting lumen area from the total area. Internal lumen radius $(r)$ was calculated from the internal circumference assuming a circular conformation of the vessel. From these parameters average media thickness $\left(M_{1}\right)$ was calculated using the following relation;

$$
M_{t}=-r+\sqrt{r^{2}+\frac{C S A}{\pi}}
$$

\section{Physiological salt solutions}

During dissection, mounting and experimentation the preparations were immersed in a Krebs bicarbonate buffered physiological salt solution (KRB). 
This solution had the following composition (in $\mathrm{mM}$ ): $\mathrm{NaCl} 118.5 ; \mathrm{KCl} \mathrm{4.7}$; $\mathrm{MgSO}_{4}$ 1.2; $\mathrm{KH}_{2} \mathrm{PO}_{4}$ 1.2; $\mathrm{NaHCO}_{3} 25.0 ; \mathrm{CaCl}_{2}$ 2.5; glucose 11.1. High potassium solution (K-KRB), was prepared by replacing all $\mathrm{NaCl}$ in normal $\mathrm{KRB}$ with an equimolar amount of $\mathrm{KCl}$. The solution used for sympathectomy of vessel segments contained normal Krebs without the addition of $\mathrm{NaHCO}_{3}$ (set at $\mathrm{pH}=4.0$ with glutathione) plus $300 \mu \mathrm{g} / \mathrm{ml}$ 6-hydroxydopamine. $\mathrm{Ca}^{2+}$-free $\mathrm{KRB}$ and $\mathrm{Ca}^{2+}$-free $\mathrm{K}-\mathrm{KRB}$ were obtained by omitting $\mathrm{CaCl}_{2}$ from $\mathrm{KRB}$ and $\mathrm{K}-\mathrm{KRB}$, respectively and including $0.3 \mathrm{mM}$ EGTA. The solutions that were used to calibrate measurements of $\left[\mathrm{Ca}^{2+}\right]_{\mathrm{i}}$ had the following composition (mM): HEPES 10.0; $\mathrm{KCl} 144.6 ; \mathrm{MgCl}_{2} \cdot 7 \mathrm{H}_{2} \mathrm{O}$ 1.17; glucose 6.0 and either $5 \mathrm{mM} \mathrm{CaCl} \cdot 2 \mathrm{H}_{2} \mathrm{O}$ or $2 \mathrm{mM}$ EGTA, for determination of $R_{\max }$ and $R_{\min }$, respectively. The medium that was used for tissue culture of small arteries contained Dulbecco's minimal Eagle's modified medium (GIBCO, Paisley, UK), $2 \mathrm{mM}$ glutamine, $100 \mathrm{IU} / \mathrm{ml}$ penicillin (Gist-Brocades, Delft, The Netherlands) and $100 \mu \mathrm{g} / \mathrm{ml}$ streptomycin (Pharmachemie, Haarlem, The Netherlands) and was supplemented with $20 \%$ dialysed fetal calf whole blood serum (Seralab, Sussex, UK).

\section{Statistical analysis}

Statistical analysis of results was performed using a statistical software package for personal computer (Crunch Interactive Statistical Package (CRUNCH, Software Corp., San Francisco, CA, USA). Depending on the experimental setup (see respective chapters) data were analysed using Student's ttest, in the case of paired comparisons or two way analysis of variance (ANOVA) followed by modified $t$-test described by Bonferroni (Wallenstein et al., 1980 ), in the case of multiple comparisons. $P<0.05$ was considered to denote statistical significance of differences. In the case of multiple comparisons, $\mathrm{P}<$ $0.05 / \mathrm{m}$ (number of comparisons $=\mathrm{m}$ ) was used to indicate statistical significance of differences. 



\section{$\alpha_{1}$-Adrenoceptor Subtype Population and Calcium Antagonist Efficacy in Large and Small Mesenteric Arteries of the Rat}

Harrie C.M. Boonen, Harry A.M.D. van der Heijden and Jo G.R. De Mey

Department of pharmacology, University of Limburg, Maastricht, the Netherlands. 


\section{SUMMARY}

We evaluated whether differences in $\alpha_{1}$-adrenoceptor subtype contribute to differences in susceptibility to calcium antagonists (CAT) between large and small arteries. Experiments were performed in segments of superior mesenteric (MA) and mesenteric resistance-sized arteries ( $\mathrm{MrA}$ ) that were i) isolated from adult rats, ii) chemically sympathectomised, iii) mounted for recording of isometric force development and iv) distended to their individual optimal lumen diameter. In both $\mathrm{MA}$ and $\mathrm{MrA}$, contractile responses to $125 \mathrm{mM}$ potassium (K-KRB) and $10 \mu \mathrm{M}$ noradrenaline (NA) consisted of an initial transient and subsequent sustained phase. Only transient responses to NA persisted in $\mathrm{Ca}^{2+}$-free solution. Pretreatment with chloroethylclonidine (CEC) reduced these in both MA and MrA. CEC did not modify sensitivity of MrA to NA in the presence of calcium, while inducing a 3-5 fold shift in MA. Responses of MrA to NA were affected more by WB 4101 than prazosin, while the affinity for both $\alpha_{1}$-adrenoceptor antagonists was comparable in MA. In MrA contractile responses to K-KRB and NA were inhibited by CAT with the following order of potency: $\mathrm{MnCl}_{2}<$ verapamil < nimodipine < felodipine (FELO). The organic CAT's were more potent in inhibiting sustained responses to K-KRB than those to NA and more potent in inhibiting sustained than transient responses to either stimulus. In MA, FELO did not display selectivity for K-KRB over NA but was still 10 times more potent in reducing sustained than transient responses to K-KRB. In general, FELO was 100-5000 times more potent in MrA than in MA. These findings indicate that in MrA maintenance of responses to NA depend primarily on $\alpha_{1 A}$-adrenoceptors while in MA $\alpha_{1 \mathrm{H}}$-adrenoceptors participate as well. In view of different signal transduction pathways linked to these adrenoceptor subtypes, this may explain relative resistance to CAT of NA-induced responsis in large arteries.

\section{INTRODUCTION}

The pharmacological properties of vascular smooth muscle are heterogenous throughout the vascular tree (De Mey and Vanhoutte, 1981; Daly et al., 1988; Heesen and De Mey, 1990). For instance, $\alpha_{1}$-adrenoceptor mediated vasoconstriction diminishes whereas postjunctional $\alpha_{2}$-adrenergic responses increase with decreasing vessel diameter along the microvascular tree of striated muscle (Faber, 1988; Messing et al., 1990). These $\alpha$-adrenoceptor types differ not only with respect to affinity for agonists and antagonists but also with respect to the signal transduction mechanism engaged (Daly et al., 1990; Michel et al., 1990). Recently, Han et al., (1987) postulated a further subclassification of $\alpha_{1}$-adrenoceptors, based on their relative sensitivity for the non-competitive antagonist chloroethylclonidine and for the competitive antagonist WB 4101. $\alpha_{1 \mathrm{~A}}$-Adrenoceptors would have a higher affinity for WB 4101 than for prazosin and would be insensitive to alkylation by chloroethylclonidine whereas $\alpha_{18}$-adrenoceptors would have similar affinity for WB 4101 and prazosin and would be non-competitively antagonised 
by chloroethylclonidine. These different $\alpha_{1}$-adrenoceptor subtypes have furthermore been postulated to couple to different calcium mobilising systems (Han et al., 1987; Wilson and Minneman, 1990). $\alpha_{1 A}$-Adrenoceptors are believed to open calcium channels in the cell membrane through pertussis toxin sensitive G-proteins while $\alpha_{1 \mathrm{~B}}$-adrenoceptors have been reported to stimulate phospholipase-C and the subsequent release of intracellular calcium (Han et al., 1987; Michel et al., 1990; Muramatsu et al., 1990; Wilson and Minneman, 1990). Recently it has been shown that in the thoracic aorta the relative contribution of $\alpha_{1 A^{-}}$and $\alpha_{1 \mathrm{~B}}$-adrenoceptors differs between different species (Oriowo and Ruffolo, 1992). Nonetheless, differences between species in effects of the dihydropyridine calcium antagonist nifedipine could not be solely explained by the presence of $\alpha_{1}$-adrenoceptor subtypes.

In isolated large arteries, calcium antagonists preferentially reduce calcium influx and contractile responses to depolarising stimuli while affecting responses to pharmacological stimulation to a lesser extent (Cauvin and Van Breemen, 1985; Cauvin et al, 1985). In small resistance sized arteries, however, which are the prime target for the antihypertensive effect of calcium antagonists (Vanhoutte, 1985; Struijker Boudier et al., 1990), this selectivity is less clear. Reports are not in agreement regarding the question whether calcium antagonists are more potent in reducing responses to depolarisation with high potassium than those to noradrenaline (Nyborg and Mulvany, 1984; Cauvin and Van Breemen, 1985; Julou and Freslon, 1986; Boonen and De Mey, 1990). The reasons for dissimilar findings are unclear. Differences in experimental setup, vascular bed and species may underlie variations in efficacy of calcium antagonists. Differences in the relative contributions of electro- and pharmaco-mechanical coupling and in the second messenger systems stimulated by $\alpha$-adrenoceptors could also be involved.

To gain more insight in mechanisms of $\alpha_{1}$-adrenoceptor mediated contractile responses and their relation with the action of different calcium antagonists, we evaluated the effects of various calcium antagonists on the initiation and maintenance of contractile responses to depolarising solution and $\alpha_{1}$-adrenergic stimulation in mesenteric arteries of different size and function. Furthermore, we assessed whether effects of calcium antagonists in large and small arteries could be explained by differences in arterial smooth muscle $\alpha_{1}$-adrenoceptor population. Findings are evaluated with respect to i) influx of extracellular and release of intracellular calcium ii) heterogeneity of $\alpha_{1}$-adrenoceptors and calcium channels and iii) interactions between electromechanical coupling and $\alpha_{1}$-adrenoceptor mediated responses.

\section{MATERIAL AND METHODS}

Experiments were performed in superior mesenteric arteries (MA) and mesenteric resistance sized arteries (MrA)(fourth order side branch of $\mathrm{MA}$ ) isolated from the mesentery of 20 week old male Wistar Kyoto rats (local inbred, University of Limburg, Maastricht, The Netherlands) that were killed by 
cervical dislocation and exsanguination. Vessels were chemically sympathectomised by 10 minute incubation at $37^{\circ} \mathrm{C}$ in bicarbonate-free Krebs solution ( $\mathrm{pH} 4.0$ ) containing $300 \mu \mathrm{g} / \mathrm{ml}$ 6-hydroxydopamine (Aprigliano and Hermsmeyer, 1977). Ring segments of the vessels $(\approx 2 \mathrm{~mm}$ length) were mounted on two stainless steel wires (diameter $40 \mu \mathrm{m}$ ) between a force transducer (Kistler Morse DSC 6, Seattle, USA) and a displacement device for recording of isometric force development (Mulvany and Halpern, 1977). Preparations were immersed in an organ bath filled with Krebs-Ringer bicarbonate buffered physiological salt solution that was maintained at $37^{\circ} \mathrm{C}$ and aerated with $95 \% \mathrm{O}_{2}$ and $5 \% \mathrm{CO}_{2}$. All arteries were distended to their individual optimal diameter $\left(\mathrm{D}_{0}\right)$ for maximal force development in response to maximal depolarisation. Preparations were therefore subjected to stepwise increases in diameter with intermittent exposure to high potassium solution (K-KRB) (De Mey and Brutsaert, 1984). Experiments with noradrenaline were performed in the continuous presence of $1 \mu \mathrm{M}$ of the $\beta$-blocking agent propranolol.

\section{Evaluation of $\alpha_{1}$-adrenoceptor subtypes}

Isolated sympathectomised segments of superior mesenteric and mesenteric resistance arteries were pretreated with $100 \mu \mathrm{M}$ chloroethylclonidine at $37^{\circ} \mathrm{C}$ during 30 minutes (Oriowo and Ruffolo, 1992). These vessels were then mounted in parallel with control vessels from the same animal. At $D_{0}$, contractile responses to noradrenaline were induced in vessels that had been incubated for 5 minutes in calcium free KRB. Thereafter, in the presence of $2.5 \mathrm{mM}$ calcium, concentration response curves to noradrenaline $(10 \mathrm{nM}-320 \mu \mathrm{M})$ were constructed in the absence or presence of either $0.1 \mu \mathrm{M} \mathrm{WB} 4101$ or $0.1 \mu \mathrm{M}$ prazosin. Finally, control responses to noradrenaline $(10 \mathrm{nM}-10 \mu \mathrm{M})$ were repeated.

\section{Effects of calcium antagonists on excitation-contraction coupling}

The contribution of intracellular calcium stores in force development following direct electromechanical or $\alpha_{1}$-adrenoceptor stimulation in vessels of different size was assessed by incubating the vessels in $\mathrm{Ca}^{2+}$-free $\mathrm{KRB}$ during 3 minutes and stimulation with either $125 \mathrm{mM} \mathrm{Ca}^{2+}$-free $\mathrm{K}-\mathrm{KRB}$ or $10 \mu \mathrm{M}$ noradrenaline. Control responses in the presence of $2.5 \mathrm{mM}$ calcium were then induced either by depolarisation of the vessels with $125 \mathrm{mM} \mathrm{K}-\mathrm{KRB}$ or by stimulation with $10 \mu \mathrm{M}$ noradrenaline during 3-5 minutes. These were repeated three times to obtain stable responses and thereafter in the presence of stepwise increasing concentrations of calcium antagonists. The dihydropyridines felodipine $(1 \mathrm{pM}-10 \mathrm{nM})$ and nimodipine $(10 \mathrm{pM}-0.1 \mu \mathrm{M}), 10 \mathrm{nM}-10 \mu \mathrm{M}$ verapamil or the inorganic calcium entry blocker $\mathrm{MnCl}_{2}(10 \mu \mathrm{M}-10 \mathrm{mM})$ were used in separate experiments. Calcium antagonists were always added, in appropriate concentrations 5 minutes prior to stimulation with K-KRB or noradrenaline. In MA this protocol was performed in the absence or presence of $0.1 \mathrm{nM}$ $30 \mathrm{nM}$ telodipine. 


\section{Solutions and drugs}

Krebs solution (KRB) had the following composition (mM): $\mathrm{NaCl}$ 118.5; $\mathrm{KCl}$ 4.7; $\mathrm{MgSO}_{4} 1.2 ; \mathrm{KH}_{2} \mathrm{PO}_{4}$ 1.2; $\mathrm{NaHCO}_{3} 25.0 ; \mathrm{CaCl}_{2}$ 2.5; glucose 11.1. K-KRB was similar to $\mathrm{KRB}$ except that all $\mathrm{NaCl}$ was replaced by an equimolar amount of $\mathrm{KCl}$. $\mathrm{Ca}^{2+}$-free $\mathrm{KRB}$ was obtained by omitting $\mathrm{CaCl}_{2}$ from normal $\mathrm{KRB}$ and including $0.3 \mathrm{mM}$ EGTA. Bicarbonate-free KRB used for sympathectomy, was set at $\mathrm{pH} 4.0$ with glutathione (Sigma). Stock solutions of the dihydropyridines felodipine, and nimodipine were dissolved in poly ethylene glycol. 6-Hydroxydopamine, noradrenaline (l-arterenol bitartrate hydrochloride), prazosin hydrochloride, dl-propranolol hydrochloride and ethylene glycol bis ( $\beta$-aminoethylether)-N-N'-tetraacetic acid (EGTA) were obtained from Sigma Chemicals (St.Louis Missouri, USA). Nimodipine was kindly supplied by Dr. S. Kazda (Bayer, FRG). Felodipine was a generous gift of Dr. M. Nordlander (Hässle, Mölndal, Sweden). Verapamil $\mathrm{HCl}$ was purchased from Knoll, Ludwigshafen, Germany. WB 4101 and chloroethylclonidine dihydrochloride were purchased from Research Biochemicals, Natick, MA, USA.

\section{Data analysis}

Sensitivities for the inhibitory effects of the calcium antagonists and for the contractile effects of noradrenaline in the absence or presence of antagonist were calculated by a least square sigmoidal curve fit of the data using a personal computer software package (Graphpad Inplot, Software, San Diego, CA, USA). Differences in selectivity of felodipine for contractions induced by high potassium and noradrenaline in large or small arteries and differences in potency and specificity of calcium antagonists to inhibit responses in MrA to depolarisation or $\alpha_{1}$-adrenoceptor stimulation were evaluated using analysis of variance followed by a t-test corrected for multiple comparisons according to the Bonferroni method (Wallenstein et al., 1980). Affinity ( $\mathrm{pA}_{2}$ ) for the competitive antagonists prazosin and WB 4101, was derived from the dissociation constant $\left(K_{d}\right)$ using the following equation:

$$
p A_{2}=-\log \left(\frac{[\text { antagonist }]}{E C_{50 Y} / E C_{50 C}-1}\right)
$$

where [antagonist] represents the molar concentration of the antagonist and $E C_{50} \mathrm{C}$ and $E C_{50} \mathrm{r}$ the sensitivities for noradrenaline in the absence and presence of antagonist, respectively. Statistical analysis of data was performed using a software package for personal computer (Crunch Interactive Statistical Package (CRUNCH, Software Corp., San Francisco, CA, USA). 


\section{RESULTS}

In denervated MrA maintained in the presence of $2.5 \mathrm{mM} \mathrm{Ca}{ }^{2+}$, responses to $125 \mathrm{mM} \mathrm{K-KRB}$ were biphasic, consisting of an initial transient $\left(K_{t}\right)$ and a subsequent sustained $\left(K_{s}\right)$ response which was maintained for several minutes (Fig. 3.1). Maximal transient responses to $125 \mathrm{mM} \mathrm{K}-\mathrm{KRB}$ averaged $2.83 \pm 0.14 \mathrm{mN} / \mathrm{mm}$ in $\mathrm{MrA}$ and $5.29 \pm 0.36 \mathrm{mN} / \mathrm{mm}$ in MA $(\mathrm{n}=12-18)$. During construction of "single dose" concentration response curves for the contractile effect of $\mathrm{K}-\mathrm{KRB}$, the transient response could progressively be discerned when MrA were stimulated with potassium concentrations above $35 \mathrm{mM}$ (Fig. 3.1).

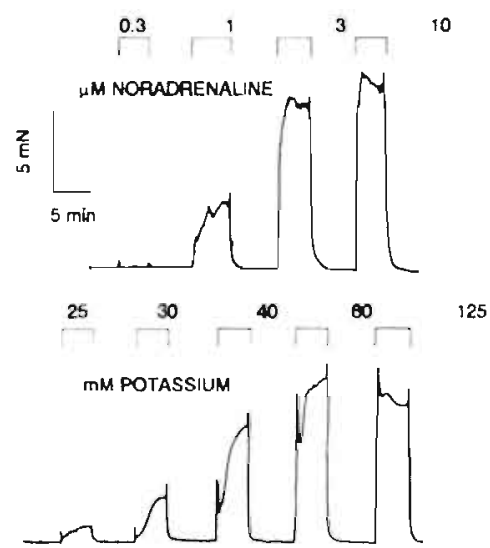

Figure 3.1. Redrawing of concentration response curves for noradrenaline and potassium in isolated, sympathectomised mesenteric resistance-sized arteries, showing apparently monophasic contractile responses to noradrenaline and the development of biphasic responses to high concentrations of potassium.

Both the transient and sustained phase of the contractile response to $125 \mathrm{mM}$ $\mathrm{K}-\mathrm{KRB}$ were rapidly lost when $\mathrm{MrA}$ and $\mathrm{MA}$ were exposed to calcium-free solution (not shown). Noradrenaline induced significantly larger contractile responses than $\mathrm{K}-\mathrm{KRB}$ in both $\mathrm{MA}$ and $\mathrm{MrA}$. These averaged $2.93 \pm 0.23$ $\mathrm{mN} / \mathrm{mm}$ in $\mathrm{MrA}$ and $8.08 \pm 0.36 \mathrm{mN} / \mathrm{mm}$ in MA $(\mathrm{n}=12)$. In the presence of $2.5 \mathrm{mM} \mathrm{Ca}^{2+}$, contractile responses to the catecholamine were apparently monophasic in MrA and MA (Fig 3.1). Following incubation in $\mathrm{Ca}^{2+}$-free KRB (containing $0.3 \mathrm{mM}$ EGTA) during 3 minutes, administration of $10 \mu \mathrm{M}$ noradrenaline induced a transient increase in wall tension in both MrA and MA (Fig. 3.3). A second or third application of noradrenaline to MrA and MA that were incubated in $\mathrm{Ca}^{2+}$-free $\mathrm{KRB}$ did not elicit any contractile response (Boonen and De Mey, 1990).

\section{$\alpha_{1}$-Adrenoceptor heterogeneity in small and large mesenteric arteries}

To evaluate the presence of subtypes of $\alpha_{1}$-adrenoceptors in $\mathrm{MrA}$ and MA, we used the antagonists chloroethylclonidine and WB 4101. Pretreatment with chloroethylclonidine did not affect maximal contractile responses to high 
potassium. These averaged $2.34 \pm 0.38 \mathrm{mN} / \mathrm{mm}$ and $2.46 \pm 0.52 \mathrm{mN} / \mathrm{mm}$ in MrA and $5.51 \pm 0.99 \mathrm{mN} / \mathrm{mm}$ and $6.22 \pm 0.96 \mathrm{mN} / \mathrm{mm}$ in MA, without and with pretreatment with chloroethylclonidine, respectively. Effects on contractile responses induced by noradrenaline in the presence of $2.5 \mathrm{mM}$ calcium after pretreatment of vessels for 30 minutes in $100 \mu \mathrm{M}$ chloroethylclonidine are shown in figure 3.2. In MA chloroethylclonidine significantly decreased the sensitivity for noradrenaline. In MrA, however, contractile responses to noradrenaline in the presence of calcium were not affected following chloroethylclonidine (Fig 3.2; Table 3.1). Yet, contractile responses that were induced by 10 $\mu \mathrm{M}$ noradrenaline in the absence of extracellular calcium were reduced by about $50 \%$ following pretreatment with chloroethylclonidine in both MA and MrA (Fig. 3.3).
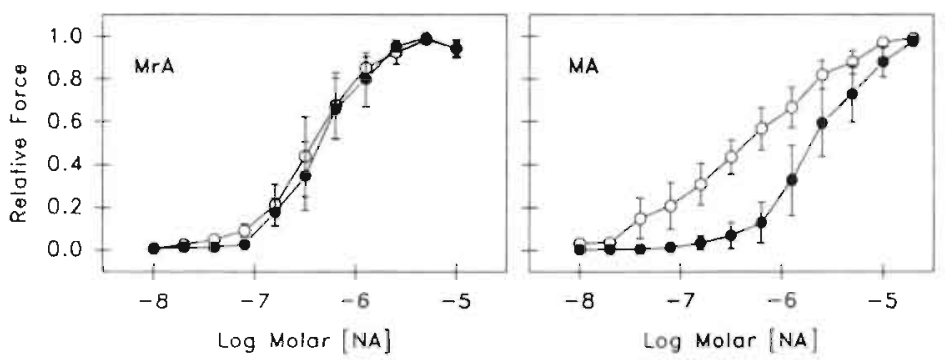

Figure 3.2. Concentration response curves for noradrenaline in isolated sympathectomised mesenteric resistance-sized arteries (MrA) and superior mesenteric artery (MA) that had been incubated in the absence (open circles) or presence (filled circles) of 100 $\mu \mathrm{M}$ chloroethylclonidine (CEC) during 30 minutes at $37^{\circ} \mathrm{C}$. Concentration response curves were performed in the presence of $1 \mu \mathrm{M}$ propranolol. Data are shown as mean \pm SEM as fraction of the maximal contractile response induced by noradrenaline in either control or CEC-pretreated vessels.

Figure 3.3. Graph showing the magnitude of contractile responses induced by noradrenaline in calcium-free solution containing $0.3 \mathrm{mM}$ EGTA as percentage of the maximal contractile response that could be obtained with noradrenaline in the presence of 2.5 $\mathrm{mM}$ calcium. Responses were obtained in control (open bars) and in vessels pretreated with chloroethylclonidine (hatched bars). Bars are means \pm SEM. * denotes statistical significance of difference (t-test, $\mathrm{P}<0.05$ ).

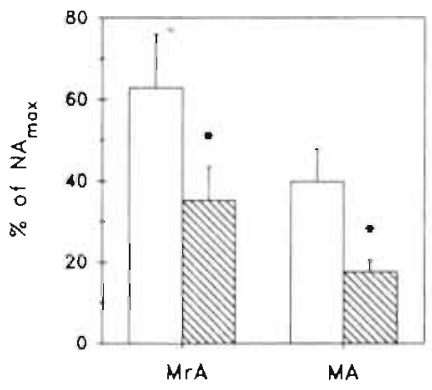


Table 3.1. Effects of $\alpha_{1}$-adrenoceptor antagonists on the sensitivity for noradrenaline in small and large mesenteric arteries.

\begin{tabular}{lcccc} 
& MrA control & MrA CEC & MA control & MA CEC \\
\hline $\mathrm{pD}_{2}$ noradrenaline & & & & \\
control & $6.42 \pm 0.12$ & $6.11 \pm 0.18$ & $6.15 \pm 0.17$ & $5.68 \pm 0.13^{b}$ \\
$+0.1 \mu \mathrm{M}$ WB 4101 & $4.27 \pm 0.13^{\mathrm{b}}$ & $4.17 \pm 0.20^{\mathrm{b}}$ & $4.77 \pm 0.12^{b}$ & $4.58 \pm 0.10^{\mathrm{b}}$ \\
$+0.1 \mu \mathrm{M}$ Prazosin & $4.65 \pm 0.09^{\mathrm{a} b}$ & $4.57 \pm 0.19^{\mathrm{b}}$ & $4.60 \pm 0.12^{\mathrm{b}}$ & $4.64 \pm 0.11^{\mathrm{b}}$ \\
$\mathrm{PA}_{2}$ & & & & \\
\hline WB 4101 & $9.15 \pm 0.14$ & $8.94 \pm 0.15$ & $8.34 \pm 0.24$ & $8.05 \pm 0.17$ \\
Prazosin & $8.77 \pm 0.14^{\mathrm{a}}$ & $8.52 \pm 0.14^{\mathrm{b}}$ & $8.53 \pm 0.19$ & $7.99 \pm 0.13$ \\
\hline
\end{tabular}

Sensitivities for noradrenaline in isolated mesenteric resistance-sized (MrA) and superior mesenteric arteries (MA) in control vessels and in vessels pretreated during 30 minutes with $100 \mu \mathrm{M}$ chloroethylclonidine (CEC) $\left(\mathrm{pD}_{2}=-\log\right.$ Molar $\left.\left[\mathrm{EC}_{50}\right]\right)$. From these the affinity $\left(\mathrm{pA}_{2}=-\log \mathrm{K}_{\mathrm{d}}\right)$ for $\mathrm{WB} 4101$ and prazosin was derived (see text). Data are means \pm SEM $(n=6)$, ${ }^{\text {t.t }}$ denote statistical significance of differences compared to WB 4101 or control values, respectively (sensitivities; ANOVA + t-test (Wallenstein, 1980), affinity; paired $t$ test).

WB 4101 and prazosin competitively antagonised contractile responses to noradrenaline in both MrA and MA (Fig 3.4; Table 3.1). The affinity for WB 4101 that was calculated from the sensitivities for noradrenaline in the absence or presence of antagonist, was larger (5-10 times) in MrA than in MA (Table 3.1). The affinity for prazosin did not differ between both types of vessel (Table 3.1). In MrA the potency of WB 4101 to inhibit responses to noradrenaline was higher than that for prazosin (Table 3.1). In MA, on the other hand, WB 4101 and prazosin were equally effective in inhibiting contractile responses to noradrenaline (Table 3.1). Pretreatment of MrA and MA with chloroethylclonidine did not significantly affect the affinity for WB 4101 and prazosin (Table 3.1).

\section{Effects of calcium antagonists in resistance arteries}

Calcium antagonists inhibited responses of MrA to $125 \mathrm{mM} \mathrm{K}-\mathrm{KRB}$ or 10 $\mu \mathrm{M}$ noradrenaline with the following order of potency: $\mathrm{MnCl}_{2}<$ verapamil < nimodipine < felodipine (Table 3.2). In general, responses to noradrenaline were less sensitive to inhibition by the calcium antagonists than responses induced by K-KRB (Table 3.2). Transient and sustained responses to both K$K R B$ and noradrenaline were affected differently by the calcium antagonists. Transient responses to both stimuli were far more resistant to inhibition than sustained responses (Table 3.2). Remarkably, the selectivity of calcium antagonists to inhibit sustained over transient responses increased with their overall 
potency, $\mathrm{MnCl}_{2}$ having the least and the dihydropyridines, particularly felodipine, having the highest selectivity (Table 3.2).
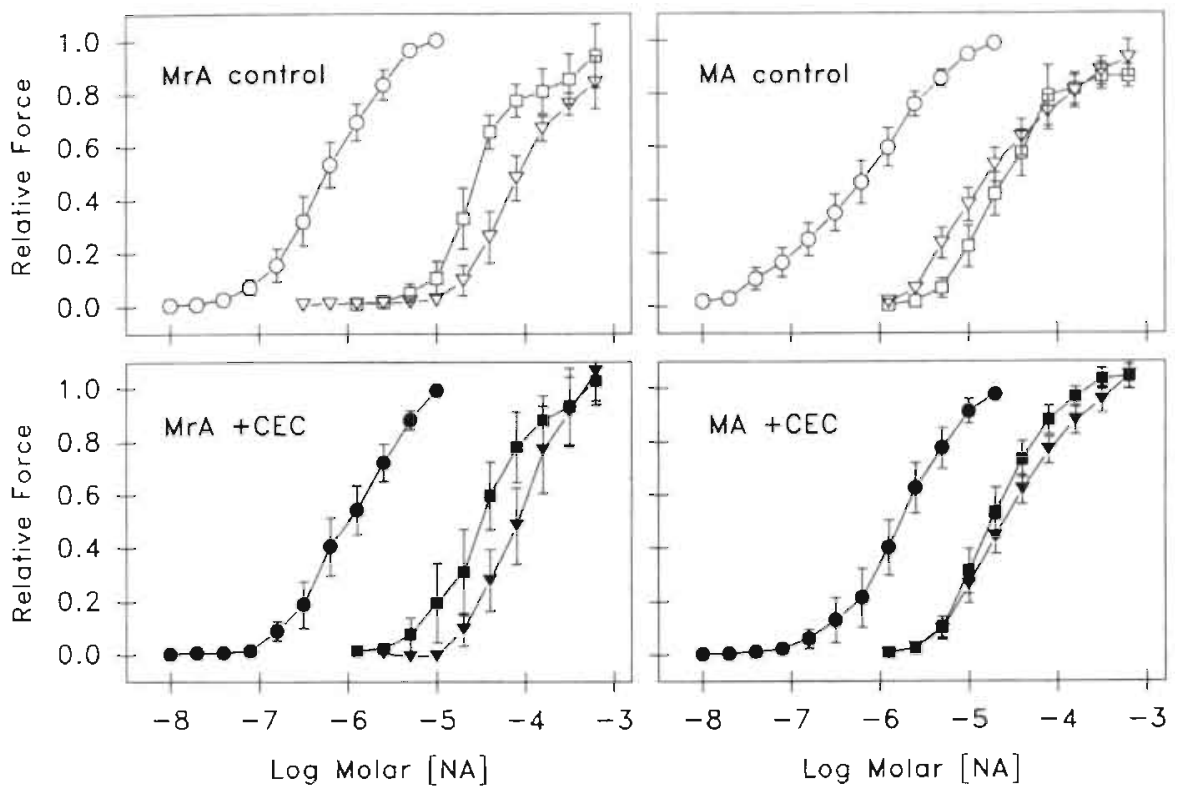

Figure 3.4. Concentration response curves for noradrenaline constructed in mesenteric resistance arteries ( $\mathrm{MrA}$ ) and in superior mesenteric arteries (MA) that had been incubated in the absence (upper panels) or presence (lower panels) of chloroethylclonidine (sce text). Concentration response curves were repeated in the presence of $0.1 \mu \mathrm{M}$ of the $\alpha_{1}$-adrenoceptor antagonists WB 4101 (triangles) and prazosin (squares). Shown are means \pm SEM as a fraction of the maximal control response.

\section{Comparison of effects of felodipine in large and small mesenteric arteries}

Figure 3.5 shows a typical tracing of an experiment in which effects of the dihydropyridine calcium antagonist felodipine were assessed in superior mesenteric artery and in mesenteric resistance sized arteries. The potency of felodipine to inhibit sustained responses to high potassium and noradrenaline in MrA was 100 to 5000 times higher than that in MA (Table 3.2). In MrA the selectivity of felodipine to inhibit sustained over transient contractile responses induced by high potassium was larger than in MA (Fig 3.5; Table 3.2). This was also the case with noradrenaline. In MA, but not in MrA, sustained contractions in response to $125 \mathrm{mM} \mathrm{KRB}$ and those evoked by $10 \mu \mathrm{M}$ noradrenaline were affected to the same extent by felodipine (Fig. 3.5; Table 3.2). 


\section{A}
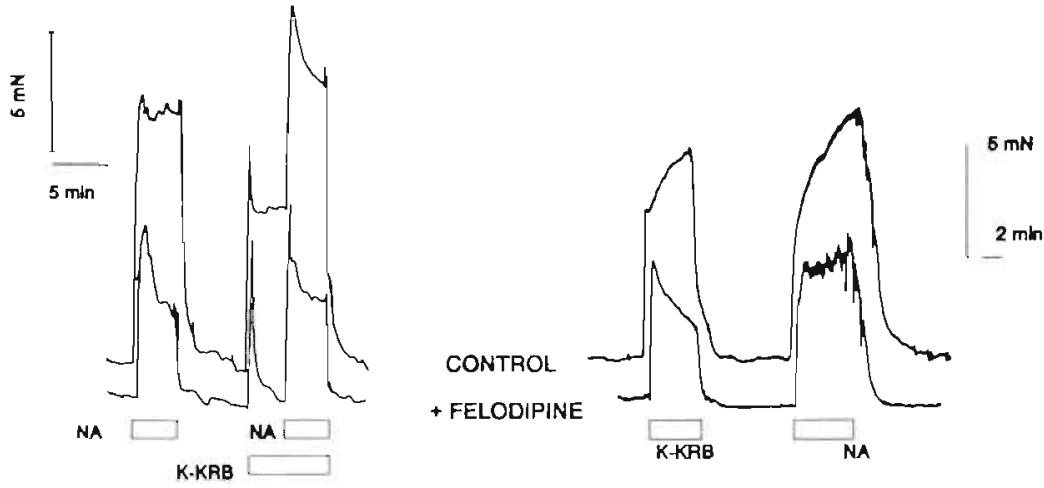

Figure 3.5. A) Superimposed redrawing of recordings showing contractile responses induced by $125 \mathrm{mM} \mathrm{K-KRB}$ and $10 \mu \mathrm{M}$ NA in MrA (left hand side) and in MA (right hand side) in the absence (upper tracings) or presence (lower tracing) of either 0.3 or 0.7 $\mathrm{nM}$ felodipine (in MrA and MA, respectively). Calibration marks represent a time scale of 5 minutes and a force development of $5 \mathrm{mN}$.

B) Concentration response curves for the dihydropyridine calcium antagonist felodipine on contractile responses induced by $125 \mathrm{mM}$ potassium (K-KRB) or $10 \mu \mathrm{M}$ noradrenaline (NA) (as is shown in Fig. $3.2 \mathrm{~A}$ ). Displayed are mean \pm SEM of transient (open symbols) and sustained (closed symbols) responses in MrA (circles $(n=7)$ ) and MA (triangles $(n=6))$.

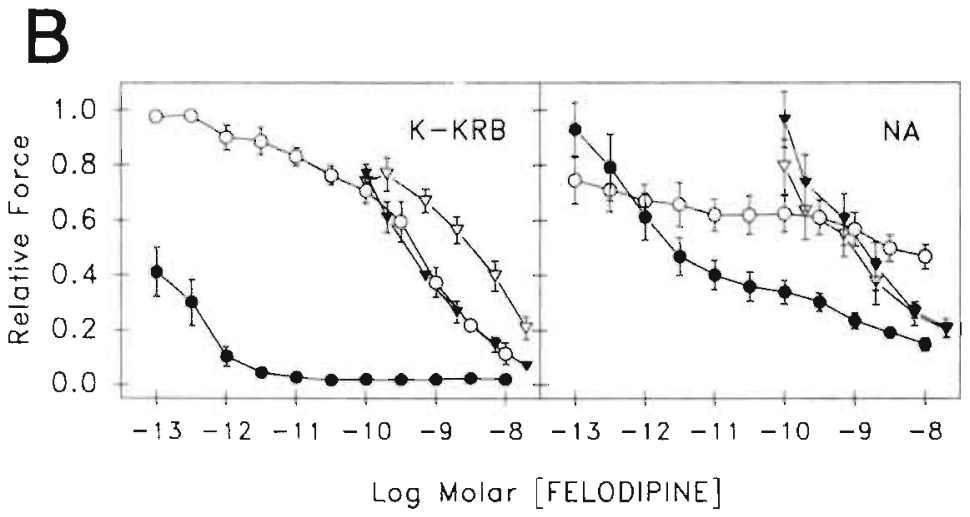


Table 3.2. Effects of calcium antagonists on contractile responses induced by potassium or noradrenaline in large and small arteries.

\begin{tabular}{lll|c}
\multicolumn{3}{c|}{$\mathrm{MrA}$} & $\mathrm{MA}$ \\
\hline $\mathrm{MnCl}_{2}$ & VERA & NIMO $\quad$ FELO & FELO
\end{tabular}

Sensitivities $\left(\mathrm{pD}_{2}\right)$

\begin{tabular}{|c|c|c|c|c|c|}
\hline$K_{1}$ & $2.57 \pm 0.03$ & $6.53 \pm 0.10$ & $8.07 \pm 0.11$ & $9.17 \pm 0.13$ & $7.97 \pm 0.30$ \\
\hline$K_{s}$ & $3.20 \pm 0.20^{a}$ & $7.86 \pm 0.17^{\mathrm{a}}$ & $10.44 \pm 0.10^{3}$ & $12.55 \pm 0.23^{\prime}$ & $9.01 \pm 0.21^{. x .5}$ \\
\hline$N A_{1}$ & $3.38 \pm 0.06^{b}$ & $6.10 \pm 0.11$ & $9.14 \pm 0.22^{\mathrm{b}}$ & $9.85 \pm 0.42$ & $9.01 \pm 0.12^{b . c}$ \\
\hline $\mathrm{NA}$ & $3.29 \pm 0.10^{b}$ & $6.75 \pm 0.13^{a . b}$ & $9.11 \pm 0.16^{\mathrm{b}}$ & $11.83 \pm 0.31^{\circ}$ & $9.08 \pm 0.23^{c}$ \\
\hline \multicolumn{6}{|l|}{ Selectivities } \\
\hline sel. $K_{1}-K_{x}$ & $0.63 \pm 0.20$ & $1.33 \pm 0.12$ & $2.37 \pm 0.11$ & $3.37 \pm 0.37$ & $1.04 \pm 0.11^{\mathrm{c}}$ \\
\hline sel. $\mathrm{K}_{-}-\mathrm{NA}_{\text {s }}$ & $-0.08 \pm 0.11$ & $1.10 \pm 0.10$ & $1.33 \pm 0.08$ & $0.71 \pm 0.28$ & $0.08 \pm 0.22^{c}$ \\
\hline
\end{tabular}

a) Sensitivities expressed as $\mathrm{pD}_{2}\left(=-\log\right.$ Molar $\left.\mathrm{IC}_{5_{0}}\right)$ of different calcium antagonists on transient $\left(_{1}\right.$ ) and sustained $\left(_{\otimes}\right.$ ) contractile responses induced by $125 \mathrm{mM}$ potassium $(\mathrm{K})$ and $10 \mu \mathrm{M}$ noradrenaline (NA) in mesenteric resistance-sized arteries (MrA) and superior mesenteric arteries (MA). b) Selectivity of calcium antagonists shown as the difference in sensitivity for inhibition of transient and sustained responses to $125 \mathrm{mM}$ potassium $\left(\mathrm{K}_{1}\right.$ $\mathrm{K}_{\mathrm{s}}$ ) and as the difference in sensitivity for potassium and noradrenaline. All values are expressed as mean \pm SEM $(n=6-7)$. ${ }^{a b}$ and ' represent significance of differences $(p<0.05)$ of trans. vs. sust., $\mathrm{K}$ vs. NA and MrA vs. MA, respectively.

\section{DISCUSSION}

Mobilisation of calcium by different mechanisms or from different sources during depolarisation and $\alpha_{1}$-adrenoceptor stimulation may affect the efficacy of calcium antagonists in large and small arteries. Stimulation of $\alpha$-adrenoceptors on vascular smooth muscle can lead to the mobilisation of calcium from intracellular stores or from the extracellular space by opening of calcium channels (Raymond et al., 1990). Recently, subtypes of $\alpha_{1}$-adrenoceptors have been proposed that differ not only in terms of affinity to specific antagonists but also with respect to calcium mobilising pathways (Muramatsu et al., 1990; Suzuki et al., 1990; Wilson and Minneman, 1990). The present study was designed to assess whether differences in efficacy of calcium antagonists in inhibiting contractile responses to depolarising solution and $\alpha_{1}$-adrenoceptor stimulation of vascular smooth muscle from large and small arteries could be explained by differences in subtypes of $\alpha_{1}$-adrenoceptors or calcium channels.

The results suggest that the relative ineffectiveness of calcium antagonists in inhibiting contractile responses to noradrenaline in large arteries can 
partly be explained by heterogeneity of $\alpha_{1}$-adrenoceptor subtypes. Furthermore, the present observations support previous suggests for a larger contribution of electro-mechanical coupling during $\alpha_{1}$-adrenoceptor mediated responses in small than in large arteries (Cauvin and Van Breemen, 1985).

\section{Characterisation of $\alpha_{1}$-adrenoceptor subtypes in mesenteric arte- ries}

In isolated mesenteric arteries, contractions induced by noradrenaline are mediated through activation of $\alpha_{1}$-adrenoceptors because in these in vitro preparations, $\alpha_{2}$-adrenergic agonists and antagonists do not display postjunctional effects (Heesen and De Mey, 1990; Eerdmans et al., 1991). In the absence of extracellular calcium, transient contractile responses could be induced by noradrenaline in both large and small arteries, strongly suggesting calcium release from intracellular stores. These responses were significantly reduced following pretreatment with chloroethylclonidine in both MA and MrA (Fig 3.3). This suggests for both vessels, the presence of $\alpha_{1 \mathrm{~B}}$-adrenoceptors that are coupled through phospholipase- $C$ to the release of intracellular calcium via generation of inositol triphosphate (Han et al., 1987; Michel et al., 1990; Muramatsu et al., 1990; Wilson and Minneman, 1990). These observations are not in agreement with others that suggest a larger contribution of release of intracellular calcium in contractile responses of large arteries than in those of small arteries (Saida and Van Breemen, 1984; Cauvin and Van Breemen, 1985). It has been suggested that in mesenteric small arteries of the rabbit, contractile responses induced by noradrenaline depend solely on influx of calcium (Cauvin et al., 1985, 1987). Yet, the present results show that in MrA of the rat, release of intracellular calcium by noradrenaline is not less marked than in MA and is in both vessel types partly sensitive to chloroethylclonidine (Fig. 3.3).

However, contractions induced by noradrenaline in the presence of calcium were sensitive to blockade by chloroethylclonidine only in large vessels (Fig. 3.3), confirming the presence of the putative $\alpha_{\mathrm{IB}}$-adrenoceptor subtype in superior mesenteric artery but not in its small resistance-sized branches. On the other hand, in MrA the affinity for WB 4101 was larger than that for prazosin, which was not the case in MA (Table 3.1). This suggests a relatively larger contribution of $\alpha_{1 A}$-adrenoceptors in MrA. This contradiction with the observations in the absence of extracellular calcium may be explained assuming that in a cumulative concentration response curve but not following a single challenge with noradrenaline, the role of calcium release in particular in MrA, is very small. Taken together, this indicates that, at least in MrA, inositol triphosphatemediated release of intracellular calcium may initiate contractile responses to high concentrations of noradrenaline, but does not contribute to the maintenance of these responses.

Arterioles have been suggested to contain only $\alpha_{2}$-adrenoceptors (Faber, 1988). On the other hand, comparing the present results with those obtained in thoracic aorta shows that the effect of chloroethylclonidine is much larger in this elastic artery than in MA (Oriowo and Ruffolo, 1992). Therefore, the following 
$\alpha$-adrenoceptor "profile" could be present along the arterial tree: descending from aorta to arterioles, $\alpha_{1 B^{-}}, \alpha_{1 A^{-}}$and $\alpha_{2}$-adrenergic responses could predominate in progressively smaller segments. Although the above mentioned observations suggest that differences exist in $\alpha_{1}$-adrenoceptor subtypes in large and small mesenteric arteries, these are certainly not absolute. In MrA, adrenergic stimulation activates predominantly $\alpha_{1 \mathrm{~A}}$-adrenoceptors but the presence of $\alpha_{1 \mathrm{~B}^{-}}$ adrenoceptors can not be excluded. Furthermore, stimulation of MA with noradrenaline results in activation of mainly the $\alpha_{1 \mathrm{~B}^{\mathrm{B}}}$-adrenoceptor subtype but $\alpha_{1 \mathrm{~A}^{-}}$ adrenoceptors may be functionally present as well.

\section{Effects of calcium antagonists in resistance arterial smooth muscle}

A vast body of evidence shows an inhibitory effect of calcium antagonists on contractile responses in arterial smooth muscle (for review see, Schwartz, 1989; Struijker Boudier et al., 1990). Effects of calcium antagonists have been shown to be mediated through binding of the drugs and interference with the function of calcium channels (Cauvin et al., 1983; Vaghy et al., 1985). In this respect, calcium channels have been proposed to be affected either by changes in membrane potential, receptor activation or stretch (Hurwitz, 1986; Bolton et al., 1988). Electrophysiological experiments demonstrated that calcium antagonists and in particular dihydropyridine calcium antagonists exert their inhibitory effect primarily on L-type voltage operated calcium channels (Hess et al., 1984; Bean et al., 1986; Nelson et al., 1990).

The present results confirm previous observations (Cauvin and Van Breemen, 1985) that the potency of dihydropyridines to inhibit sustained responses to high potassium is higher than that of non-dihydropyridine calcium antagonists (Table 3. 2). However, transient responses to potassium were, in relative terms, increasingly resistant to manganese, verapamil and dihydropyridines (Table 3.2). These differences in effects of calcium antagonists on initial transient and sustained responses to potassium could reflect activation of different calcium channel types (Rusch and Hermsmeyer, 1988) or calcium channel states (Hess et al., 1984; Bean et al., 1986; Boonen and De Mey, 1990).

Susceptibility of contractile responses induced by noradrenaline to calcium antagonists suggests a strong involvement of voltage operated calcium channels in the $\alpha_{1}$-adrenoceptor mediated response in resistance arterial smooth muscle. This can either be explained by the depolarising effect of noradrenaline in these vessels (Mulvany et al., 1982; Hermsmeyer, 1983) or by direct effects of noradrenaline on voltage operated calcium channels (Nelson et al., 1988; Boonen and De Mey, 1990b). Regardless the precise mechanism, activation of L-type calcium channels in MrA by noradrenaline is strengthened by the observation that the potency of calcium antagonists to inhibit these responses increases from inorganic calcium antagonist to the dihydropyridines which have the highest specificity to inhibit L-type voltage operated calcium channels (Vaghy et al., 1985; Hurwitz, 1986). 


\section{Comparison of excitation-contraction coupling in large and small mesenteric artery}

Sensitivities for the inhibitory effect of felodipine differed markedly between large and small mesenteric arteries (Fig. 3.5). Sustained contractile responses induced by potassium as well as those induced by noradrenaline were 100 - 5000 times more sensitive to inhibition by felodipine in MrA than in MA (Table 3.2). Yet, also in large mesenteric arteries, sustained responses were affected more by felodipine than the initial transient responses to stimulation with potassium and noradrenaline (Fig. 3.5). The different effects of felodipine on contractile responses induced by stimulation with noradrenaline may be explained by differences in calcium mobilising pathways that are activated by different $\alpha_{1}$-adrenoceptor subtypes in large and small arteries. The large difference in the efficacy of felodipine to inhibit contractile responses to potassium in large and small arteries, however, can not be fully explained from the present results. They may be due to differences in calcium channel characteristics or could reflect differences in additional intracellular calcium mobilising pathways in MA and MrA that may be activated by calcium influx itself (Berridge and Galione, 1988; Van Breemen and Saida, 1989). Also in the microcirculatory bed of striated muscle, the efficacy of felodipine increases with decreasing arteriolar diameter (Messing et al., 1991). Differences in calcium sensitivity of the contractile apparatus are possibly not causing the different effects of felodipine in the different arteries. If anything, calcium sensitivity has been shown to be higher in small than in large mesenteric arteries (Boels et al., 1991).

The present results suggest that the distribution of $\alpha_{1}$-adrenoceptor subtypes changes from large to small mesenteric arteries. Large arteries contain mainly the $\alpha_{1 \mathrm{~B}}$-adrenoceptor subtype whereas in resistance sized arteries a growing contribution of $\alpha_{1 \mathrm{~A}}$-adrenoceptors appears. Consequently, the contribution of calcium influx in the maintenance of the contractile response following $\alpha_{1}$-adrenergic stimulation decreases with vessel diameter. This distribution of $\alpha_{1}$-adrenoceptor subtypes may explain differences in susceptibility for dihydropyridine calcium antagonists during $\alpha_{1}$-adrenoceptor stimulation between vessels of different size. However, differences in the inhibitory action of calcium antagonists on contractions induced by direct stimulation of large and small arteries can not be explained by the present findings. 


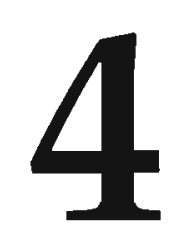

\section{Increased Calcium Sensitivity in Isolated Resistance Arteries from Spontaneously Hypertensive Rats: Effects of Dihydropyridines}

Harrie C.M. Boonen and Jo G.R. De Mey

Department of pharmacology, University of Limburg, Maastricht, the Netherlands.

(Eur. J. Pharmacol. 1990, 179: 403-412) 


\section{SUMMARY}

We recorded the contractile responses to calcium in mesenteric resistance arteries of Wistar Kyoto (WKY) and spontaneously hypertensive rats (SHR) during depolarisation or stimulation with noradrenaline. The effects of Bay-K8644 and nimodipine on these responses were evaluated. Calcium sensitivity was higher in noradrenaline stimulated than in depolarised vessels. Nimodipine decreased, and Bay-K8644 increased calcium sensitivity. Both were more potent in the presence of potassium than in the presence of noradrenaline. Calcium sensitivity was higher in SHR than in WKY vessels only during stimulation with noradrenaline. Rhythmic responses, observed in SHR vessels during stimulation with noradrenaline, were abolished by nimodipine. In WKY vessels, rhythmicity could be induced by Bay-K8644. Modulation of calcium sensitivity by dihydropyridines during electrochemical as well as pharmacological stimulation suggests that in resistance arterial smooth muscle the function of voltage-operated calcium channels can be modulated by noradrenaline. This modulation could differ quantitatively between mesenteric resistance arteries of SHR and WKY.

\section{INTRODUCTION}

Altered cellular handling of calcium ions could be involved in the hypersensitivity and hyperreactivity to vasoconstrictors and in the hypertrophy observed in the arteries of hypertensive humans and experimental animals (Mulvany and Nyborg, 1980; Mulvany, 1983; Lever, 1986; Sharma and Bhalla, 1988; Bolzon and Cheung, 1989). At least two types of stimuli, depolarisation and vasoconstrictor agents increase the influx of extracellular calcium into arterial smooth muscle cells (Berridge, 1986; Van Breemen et al., 1986). Effects of noradrenaline and depolarisation induced by a high external concentration of potassium are additive (Van Breemen et al, 1986) and differ in their susceptibility to dihydropyridines (Hess et al., 1984; Bean et al., 1986). As a consequence, the existence of two separate calcium influx pathways has been proposed: receptor- and voltage-operated calcium channels (Van Breemen et al., 1972; Bolton, 1979). The existence of the latter has been confirmed by patch-clamp experiments (Hurwitz, 1986). Voltage-insensitive calcium channels activated by noradrenaline, have so far not been identified. Recent electrophysiological observations suggest that noradrenaline could affect the function of voltage-operated calcium channels (Benham and Tsien, 1988; Nelson et al., 1988). Observations in multicellular preparations that are in line with this suggestion and with the possibility that this process might be involved in contractile responses to noradrenaline and in the hypersensitivity to calcium in resistance arteries of hypertensive rats are sparse.

In this study we therefore recorded contractile responses to depolarisation, noradrenaline and calcium in isolated mesenteric resistance-sized arteries of normotensive Wistar-Kyoto and spontaneously hypertensive rats and analysed effects of the dihydropyridine calcium agonist and antagonist, Bay-K8644 and nimodipine, on these responses. The effects are evaluated with respect to 
the above mentioned hypothesis of adrenergic modulation of calcium channel function.

\section{MATERIAL AND METHODS}

The experiments were performed in mesenteric resistance arteries of 20 week old male Wistar Kyoto rats and age matched spontaneously hypertensive rats (SHR, local inbred strain). The rats were killed by a sharp blow on the head and exsanguination. A loop of small intestine and mesentery was isolated and immersed in Krebs-Ringer bicarbonate (KRB) solution. Segments (1.5 - 2 $\mathrm{mm}$ long) of fourth order side branches of the superior mesenteric artery were isolated from the proximal part of the small intestine. To destroy adrenergic nerve endings in the vessel wall, resistance arterial preparations were incubated for 10 minutes in bicarbonate-free $\mathrm{KRB}$ containing $300 \mu \mathrm{g} / \mathrm{ml}$ 6-hydroxydopamine (Aprigliano and Hermsmeyer, 1977). The segments were then threaded onto two stainless steel wires (diameter $0.04 \mathrm{~mm}$ ) and mounted as ring segments between a Kistler-Morse DSC 6 (Seattle, USA) force transducer and a displacement device in an isometric myograph (Mulvany and Halpern, 1977). Arteries of WKY and SHR were mounted in the same organ chamber and were studied in parallel.

During dissection, mounting and experimentation, the preparations were immersed in $\mathrm{KRB}$ that was maintained at $37^{\circ} \mathrm{C}$ aerated with $95 \% \mathrm{O}_{2}-5 \% \mathrm{CO}_{2}$. The Krebs-Ringer solution had the following composition $(\mathrm{mM}): \mathrm{NaCl} 118.5$; $\mathrm{KCl}$ 4.7; $\mathrm{MgSO}_{4}$ 1.2; $\mathrm{KH}_{2} \mathrm{PO}_{4}$ 1.2; $\mathrm{NaHCO}_{3}$ 25.0; $\mathrm{CaCl}_{2}$ 2.5; glucose 11.1. High potassium solution (K-KRB), was prepared by replacing all the $\mathrm{NaCl}$ in normal $\mathrm{KRB}$ with an equimolar amount of $\mathrm{KCl}$. $\mathrm{Ca}^{2+}$-free $\mathrm{KRB}$ and $\mathrm{Ca}^{2+}$-free $\mathrm{K}-\mathrm{KRB}$ were obtained by omitting $\mathrm{CaCl}_{2}$ from $\mathrm{KRB}$ and $\mathrm{K}-\mathrm{KRB}$, respectively and including $0.3 \mathrm{mM}$ EGTA.

The pharmacological agents that were used included 6-hydroxydopamine, l-noradrenaline hydrochloride (NA) and ethylene glycol bis ( $\beta$-aminoethyl-ether)-N-N'-tetraacetic acid (EGTA) which were obtained from Sigma (St.Louis Missouri, USA). Nimodipine and BAY-K8644 were kindly supplied by Dr. S. Kazda (Bayer, FRG).

The experiments were performed with each preparation stretched to its optimal lumen diameter for mechanical performance determined by a lengthtension protocol that was previously described (De Mey et al., 1987).

Maximal contractile responses to noradrenaline $(10 \mu \mathrm{M})$, K-KRB (125 $\mathrm{mM})$ and to the combination of noradrenaline $(10 \mu \mathrm{M})$ and K-KRB (125 mM) were obtained in the continuous presence of $2.5 \mathrm{mM}$ calcium. Then calcium concentration-response curves were obtained as follows. The vessels were exposed for $3 \mathrm{~min}$ to $\mathrm{Ca}^{2+}$-free $\mathrm{KRB}$ and were depleted of intracellular calcium by two consecutive stimulations with $10 \mu \mathrm{M}$ noradrenaline. Cumulative concentration-response curves for calcium ( $10 \mu \mathrm{M}$ to $10 \mathrm{mM}$ ) were subsequently constructed during continuous exposure to noradrenaline $(10 \mu \mathrm{M})$, K-KRB (125 
$\mathrm{mM}$ ) or $10 \mu \mathrm{M}$ noradrenaline plus $125 \mathrm{mM} \mathrm{K}-\mathrm{KRB}$. After 20 minutes of recovery in $\mathrm{KRB}$ containing $2.5 \mathrm{mM} \mathrm{CaCl}$ the vessels were exposed to nimodipine $(10 \mathrm{pM}-10 \mathrm{nM})$ or Bay-K8644 (1 -100 $\mathrm{nM})$ and calcium concentration-response curves were repeated in the presence of stimulus and dihydropyridine. For all concentration-response curves the negative logarithm of the calcium concentration that was required to induce $50 \%$ of the maximal contractile response $\left(p C \mathrm{~A}_{2}\right)$ was determined.

Amplitudes of contraction were expressed as increase in wall tension (increase in force divided by twice the vessel segment length). All data are shown as means \pm SEM with $n$ indicating the number of observations. Statistical significance of differences between WKY and SHR was evaluated by Student's t-test for unpaired observations. Changes in contractile responses and sensitivities to calcium from control following exposure to the dihydropyridines were analysed using analysis of variance followed by modified $t$-statistics according to the method described by Bonferroni (Wallenstein et al., 1980) in the case of multiple comparisons. Only statistically significant differences are described ( $P<0.05$ denotes statistical significance of differences).

\section{RESULTS}

Optimal lumen diameter of mesenteric resistance arteries did not differ between WKY $(185 \pm 27 \mu \mathrm{m}, \mathrm{n}=37)$ and SHR $(197 \pm 39 \mu \mathrm{m}, \mathrm{n}=37)$. Responses of sympathectomised vessels to $125 \mathrm{mM} \mathrm{K}-\mathrm{KRB}$ consisted of an initial transient and a subsequent sustained phase (Fig. 4.1).

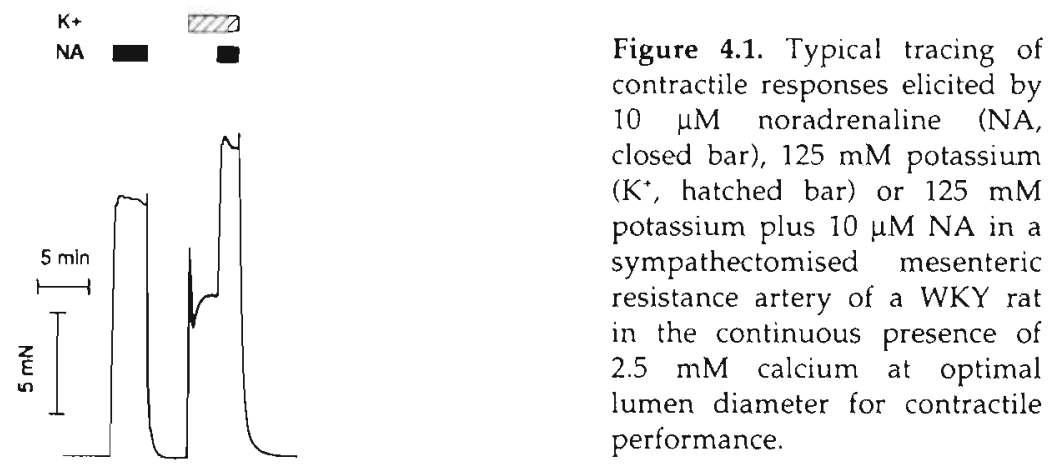

The amplitudes of the sustained responses were larger in SHR $(2.75 \pm 0.14$ $\mathrm{mN} / \mathrm{mm}, \mathrm{n}=37)$ than in $W K Y(1.89 \pm 0.10 \mathrm{mN} / \mathrm{mm}, \mathrm{n}=37)$. Unlike responses to $125 \mathrm{mM} \mathrm{K-KRB}$, those to a single administration of a maximal concentration of noradrenaline $(10 \mu \mathrm{M})$ were apparently monophasic (Fig. 4.1). SHR vessels responded to $10 \mu \mathrm{M}$ noradrenaline with a larger contraction (3.62 \pm 0.16 $\mathrm{mN} / \mathrm{mm} \mathrm{n}=37)$ than $W K Y$ vessels $(2.58 \pm 0.12 \mathrm{mN} / \mathrm{mm} \mathrm{n}=37)$. Contractile 
responses to noradrenaline plus K-KRB (Fig. 4.1) were significantly larger in all vessels than those to either stimulus alone. Again they were greater with SHR $(4.52 \pm 0.20 \mathrm{mN} / \mathrm{mm} \mathrm{n}=37)$ than with WKY $(3.39 \pm 0.17 \mathrm{mN} / \mathrm{mm} \mathrm{n}=37)$ vessels.

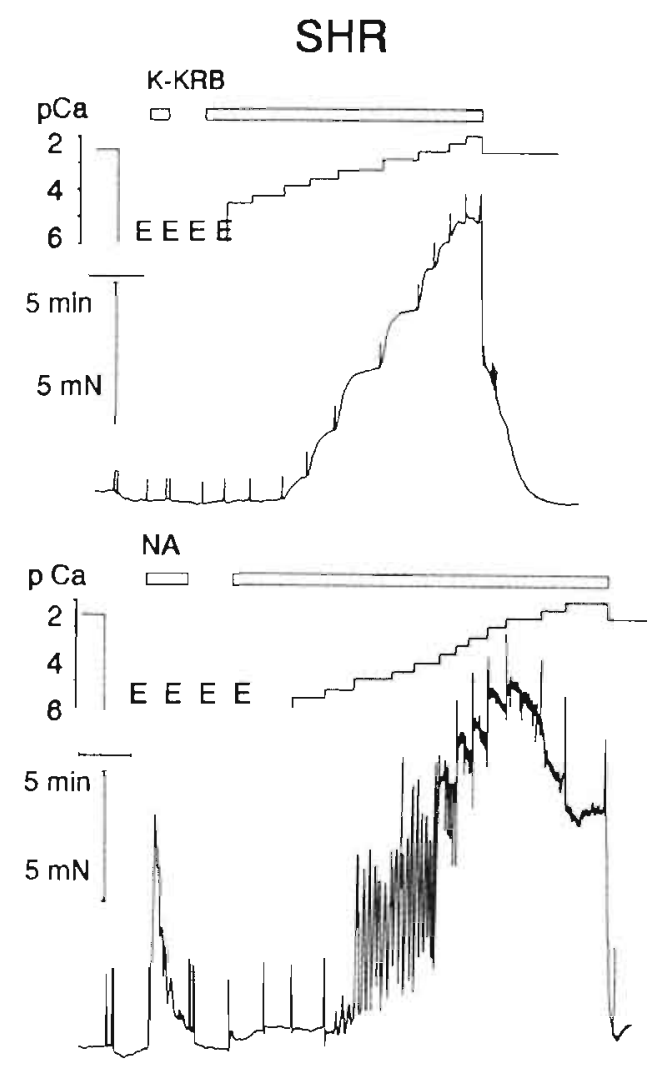

Figure 4.2. Typical tracings of concentration response curves for calcium constructed in calcium depleted mesenteric resistance arteries of SHR rats. E represents incubation of the artery in calcium free KRB with $0.3 \mathrm{mM}$ EGTA. Hatched and open bars indicate the presence of 125 $\mathrm{mM}$ K-KRB or $10 \mu \mathrm{M}$ noradrenaline, respectively. $\mathrm{pCa}$ represents the negative logarithm of the calcium concentration present in the organ bath.

\section{Responses to calcium in resistance arteries of Wistar Kyoto rats}

No contractile response to $\mathrm{K}-\mathrm{KRB}$ could be obtained following the removal of extracellular calcium. Under these conditions however, 10 $\mu \mathrm{M}$ noradrenaline induced a single transient response that averaged $46 \pm 5 \%$ of the response in the presence of $2.5 \mathrm{mM}$ calcium. Administration of calcium induced concentration-dependent tonic contractions in resistance-sized arteries that had been depleted of intracellular calcium and exposed to $\mathrm{K}-\mathrm{KRB}$, noradrenaline or noradrenaline plus K-KRB. Sensitivity to the divalent cation was larger in the presence of noradrenaline than during K-KRB stimulation (Table 4.1). Calcium sensitivity was even larger in the presence of a combination of noradrenaline and K-KRB (Table 4.1). It is especially interesting that concentrations of calcium which were below the threshold needed to induce contraction in the presence of either stimulus alone, could induce up to $50 \%$ of the maximal contractile response in the presence of a combination of both stimuli (Fig. 4.3). Concentrations of calcium exceeding $2.5 \mathrm{mM}$ reduced 
contractile responses in the presence of noradrenaline but not in the presence of K-KRB (Fig. 4.2 and 4.3).

Table 4.1. Effects of nimodipine and Bay-K 8644 on calcium sensitivity $\left(\mathrm{pCa}_{2}\right)$ in mesenteric rmistance arteries of WKY and SHR ?

\begin{tabular}{|c|c|c|}
\hline & \multicolumn{2}{|c|}{$\mathrm{pCa}_{2}(-\log [$ Molar $])$} \\
\hline & WKY & SHR \\
\hline $\mathrm{K}-\mathrm{KRB} 125 \mathrm{mM}$ & $3.20 \pm 0.03$ & $3.33 \pm 0.04$ \\
\hline+ NIMO $10 \mathrm{pM}$ & $3.01 \pm 0.05^{\circ}$ & $3.05 \pm 0.05^{b}$ \\
\hline+ NIMO $0.1 \mathrm{nM}$ & $2.84 \pm 0.02^{b}$ & $2.82 \pm 0.03^{b}$ \\
\hline$+\mathrm{NIMO} 1.0 \mathrm{nM}$ & $2.71 \pm 0.03^{b}$ & $2.68 \pm 0.02^{b}$ \\
\hline + Bay-K 8644 & $3.93 \pm 0.03^{\circ}$ & $4.05 \pm 0.04{ }^{b x}$ \\
\hline NA $10 \mu \mathrm{M}$ & $3.87 \pm 0.04$ & $4.44 \pm 0.10^{c}$ \\
\hline+ NIMO $0.1 \mathrm{nM}$ & $3.46 \pm 0.06^{b}$ & $3.87 \pm 0.08^{b c}$ \\
\hline+ NIMO $1.0 \mathrm{nM}$ & $3.15 \pm 0.05^{b}$ & $3.26 \pm 0.07^{b}$ \\
\hline$+\mathrm{NIMO} 10 \mathrm{nM}$ & $2.86 \pm 0.04^{b}$ & $2.88 \pm 0.05^{\circ}$ \\
\hline NA $10 \mu \mathrm{M}$ & $3.90 \pm 0.12$ & $4.38 \pm 0.20^{\mathrm{c}}$ \\
\hline+ Bay-K 86441 nM & $3.84 \pm 0.05$ & $4.32 \pm 0.09^{c}$ \\
\hline+ Bay-K $864410 \mathrm{nM}$ & $4.21 \pm 0.15^{b}$ & $4.36 \pm 0.06$ \\
\hline + Bay-K $8644100 \mathrm{nM}$ & $4.10 \pm 0.06^{b}$ & $4.20 \pm 0.07$ \\
\hline$N A+K-K R B$ & $4.25 \pm 0.07$ & $4.49 \pm 0.06^{c}$ \\
\hline
\end{tabular}

Mean values ( $n=6)$ and SEM of calcium concentrations needed to obtain a halfmaximal contractile response. ${ }^{\text {b.c }}$ Denote statistical significance of difference from control or WKY, respectively $(\mathrm{P}<0.05)$.

\section{Effects of dihydropyridines in resistance arteries of Wistar Kyoto rats}

Nimodipine (10 pM to $10 \mathrm{nM}$ ) did not affect resting wall tension of the vessels. It reduced sensitivity (Table 4.1) and maximal responses (Fig, 4.4) to calcium in stimulated vessels. Nimodipine was more potent in K-KRB than in noradrenaline stimulated vessels (Fig. 4.4, Table 4.1). Nimodipine did not affect relaxing responses to millimolar concentrations of calcium in noradrenaline stimulated vessels. 


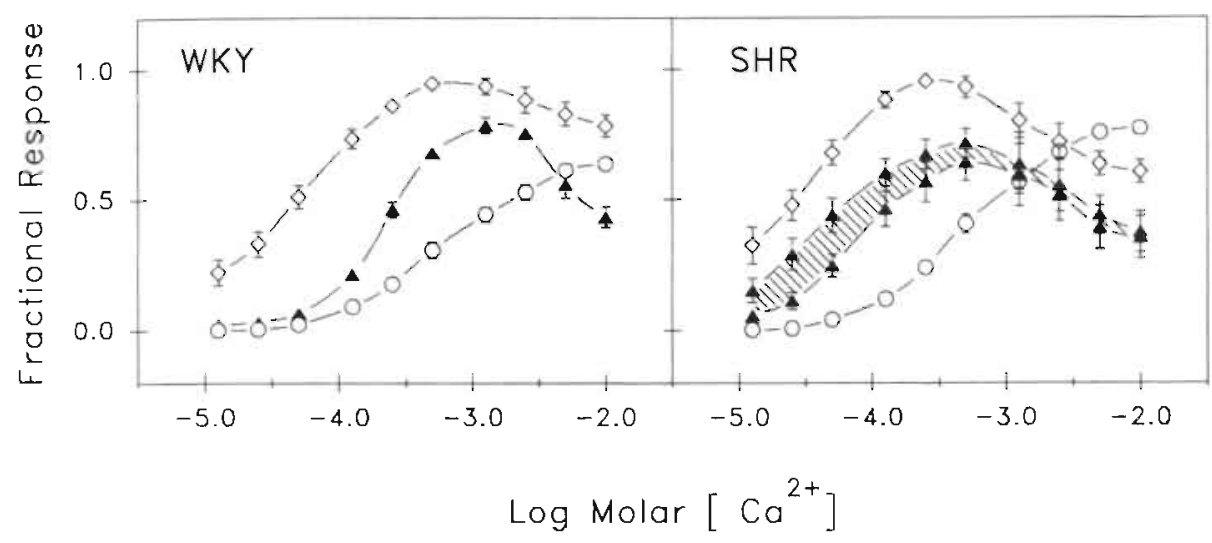

Figure 4.3. Concentration response curves for calcium in mesenteric resistance arteries of WKY (left) and SHR (right) rats. The curves were constructed in the continuous presence of $125 \mathrm{mM}$ potassium (open circle), $10 \mu \mathrm{M}$ noradrenaline (closed triangle) or $125 \mathrm{mM}$ potassium $+10 \mu \mathrm{M}$ noradrenaline (open diamond). Shown are mean values $\pm \mathrm{SEM}(\mathrm{n}=$ 6) as fraction of the maximal response to a combination of $125 \mathrm{mM}$ potassium and $10 \mu \mathrm{M}$ noradrenaline in the presence of $2.5 \mathrm{mM}$ calcium. The hatched area indicates rhythmicity.

Bay-K8644 $(100 \mathrm{nM})$ induced a contraction that, unlike those to K-KRB and noradrenaline, consisted of rhythmic oscillations superimposed on a tonic increase in force in the majority of the vessels (Fig. 4.5). Both components were readily abolished when in the presence of $2.5 \mathrm{mM}$ calcium, the extracellular potassium concentration was slightly increased (from 5.9 to 10 or $15 \mathrm{mM}$; Fig. 4.5), and upon removal of extracellular calcium (Fig. 4.5). Bay-K8644 significantly increased the sensitivity to calcium in vessels that were exposed to 125 $\mathrm{mM}$ K-KRB. In vessels exposed to noradrenaline, Bay-K8644 increased the calcium sensitivity only marginally but significantly (Table 4.1 and Fig. 4.6). However, Bay-K8644 had no significant effect on maximal contractile responses to calcium in the presence of either $125 \mathrm{mM} \mathrm{K-KRB}$ or $10 \mu \mathrm{M}$ noradrenaline (Fig. 4.6). Neither did this dihydropyridine affect the relaxing effects of supramillimolar concentrations of calcium in vessels exposed to noradrenaline (Fig. 4.6).

\section{Observations in resistance arteries of spontaneously hyperten- sive rats}

Removal of extracellular calcium affected SHR vessels to the same extent as WKY vessels. It abolished contractile responses to K-KRB and reduced the responses to noradrenaline to a single transient one that reached $42 \pm 5 \%$ of control (Fig. 4.2) ( $P>0.05$ versus WKY). Like WKY vessels, SHR vessels were least sensitive to calcium in depolarising solution, intermediately sensitive in the presence of $10 \mu \mathrm{M}$ noradrenaline and most sensitive to calcium when ex- 


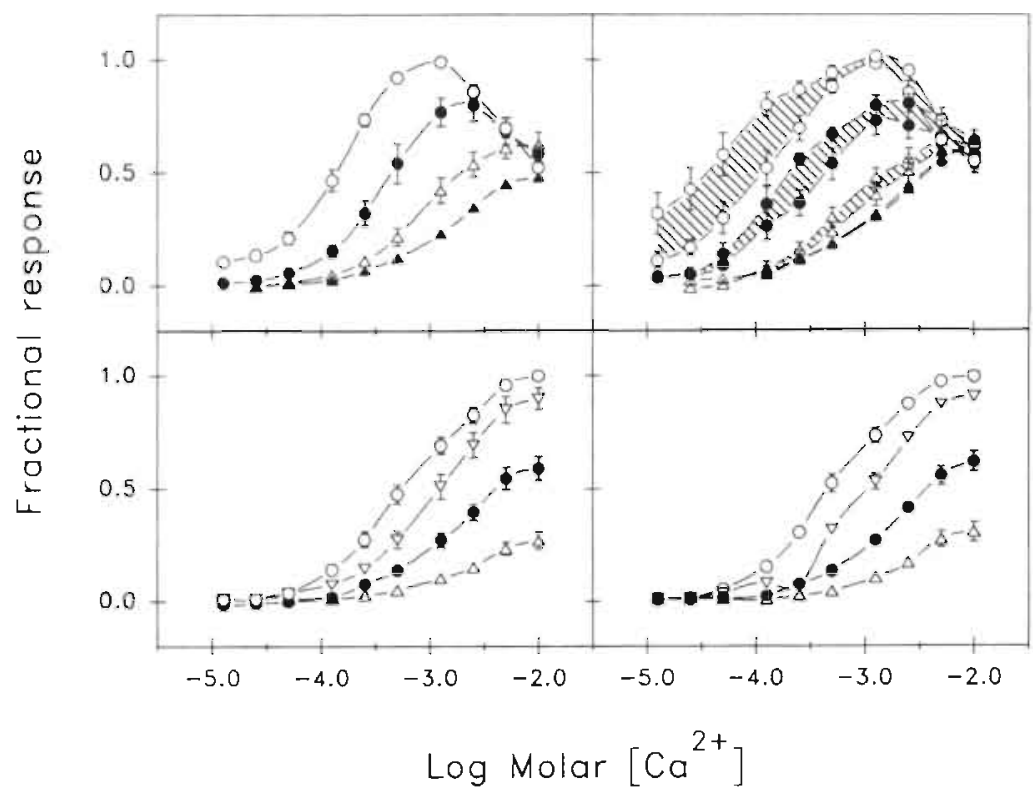

Figure 4.4. Concentration response curves for calcium in mesenteric resistance arteries of WKY (left) and SHR (right) rats. The vessels were exposed to $10 \mu \mathrm{M}$ noradrenaline (upper panels) to $125 \mathrm{mM}$ potassium (lower panels) in the absence (open circle) or presence of nimodipine; $10 \mathrm{pM}$ (open inverted triangle), $0.1 \mathrm{nM}$ (filled circle), $1 \mathrm{nM}$ (open triangle) or $10 \mathrm{nM}$ (filled triangle), respectively. Shown are means \pm SEM as fraction of the maximal response in the absence of nimodipine. Hatched areas represent rhythmic responses.

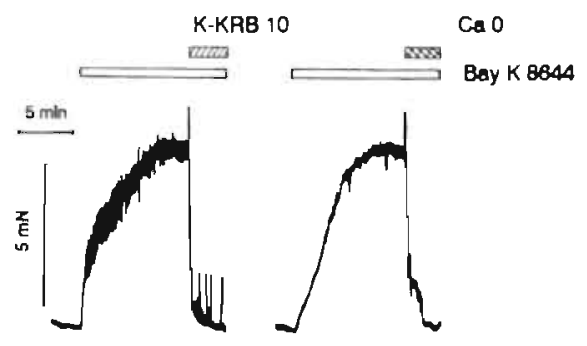

Figure 4.5. Typical tracings of response's to 100 n.M Bay-K8644 (open bars) in mesenteric resistance arteries of a WKY rat. Addition of $10 \mathrm{mM}$ potassium (hatched bar) or depletion of extracellular calcium (crossed bar) abolished the contractile responses to Bay-K8644.

posed to both K-KRB and noradrenaline (Table 4.1 and Fig. 4.3). While WKY and SHR vessels were equally sensitive to calcium in depolarising solution, calcium sensitivity in the presence of either $10 \mu \mathrm{M}$ noradrenaline alone or both $\mathrm{K}-\mathrm{KRB}$ and noradrenaline was significantly higher in SHR than in WKY vessels (Table 4.1). An additional point of interest is that, unlike the situation in WKY vessels, contractile responses of SHR vessels to calcium in the presence of 10 $\mu \mathrm{M}$ noradrenaline were composed of rhythmic oscillations superimposed on 


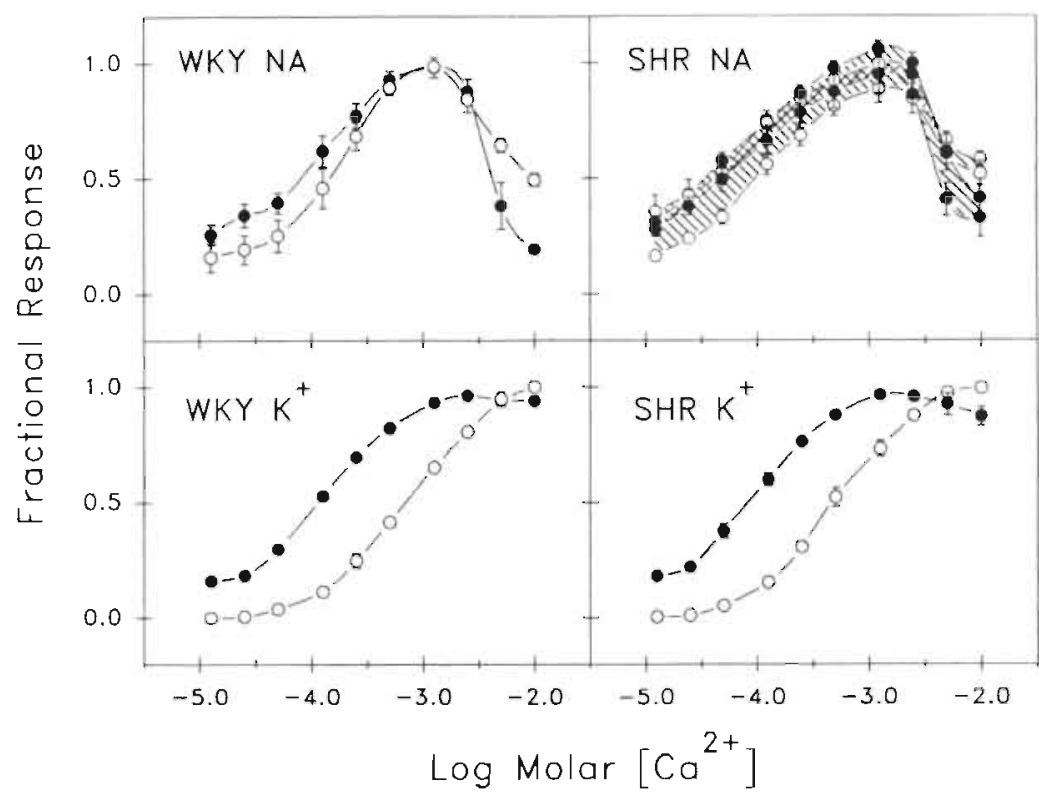

Figure 4.6. Concentration response curves for (alcium in mesenteric resistance arteries of WKY (left) and SHR (right) rats, exposed to $10 \mu \mathrm{M}$ noradrenaline (upper panels) or 125 $\mathrm{mM}$ potassium (lower panels) in the absence (open circle) or in the presence of $10 \mathrm{nM}$ BayK8644 (closed circle). Shown are mean values \pm SEM $(n=7)$ as a fraction of the maximal response in the absence of Bay-K8644. Hatched areas represent rhythmic responses.

tonic increases in wall tension (Fig. 4.2). With increasing extracellular calcium concentration the amplitude of this rhythmicity decreased and the frequency increased, respectively. In order to visualize rhythmicity, the area between sustained and maximal rhythmic response is striated in the figures $4.3,4.4$ and 4.6. Despite differences in contractile responsiveness to calcium in vessels exposed to noradrenaline, the relaxing effects of high concentrations of calcium in the presence of the catecholamine did not differ between WKY and SHR.

Effects of nimodipine in SHR resistance arteries were qualitatively similar to those observed in WKY vessels. The dihydropyridine reduced responses to calcium. As in WKY vessels, nimodipine was more potent in this respect in vessels exposed to $125 \mathrm{mM} \mathrm{K-KRB}$ than in the presence of noradrenaline (Fig. 4.4). However, nimodipine abolished the differences that were observed between SHR and WKY vessels in calcium sensitivity and in the occurrence of rhythmicity (Table 4.1).

Administration of a single concentration of Bay-K8644 (100 nM) induced similar contractions in SHR and WKY vessels. Bay- K8644 increased the sensitivity for calcium in vessels that were exposed to $K-K R B$ but not, unlike in WKY vessels, in those exposed to noradrenaline (Fig. 4.6, Table 4.1). Bay-K8644 
induced a minimal but significant difference in calcium sensitivity between WKY and SHR vessels exposed to K-KRB. It eliminated on the other hand, the difference in calcium sensitivity between $W K Y$ and SHR vessels stimulated with noradrenaline (Table 4.1). Bay-K8644 affected neither maximal contractile responses to calcium nor did it affect rhythmic responses to calcium in vessels exposed to noradrenaline.

\section{DISCUSSION}

The present observations on resistance arteries are compatible with direct indications that noradrenaline can affect the function of voltage-operated calcium channels in arterial smooth muscle. This mechanism could explain differences in pharmaco- and electro-mechanical coupling and differences in calcium handling between resistance arteries of normotensive and spontaneously hypertensive rats.

\section{Mechanisms involved in calcium handling}

Maximal contractile responses of mesenteric resistance arteries to noradrenaline and depolarisation were partly additive (Fig. 4.1). Since this also applies to stimulated calcium influx (Hurwitz, 1986), the existence in the sarcolemma of separate sets of receptor- and voltage-operated calcium channels has been suggested (Van Breemen et al., 1972; Bolton, 1979). While voltage-operated calcium channels have been identified by patch clamp techniques (Hurwitz, 1986), there is no direct evidence for the existence in arterial smooth muscle cells of voltageinsensitive calcium channels that are activated by noradrenaline. Instead recent studies suggest that noradrenaline can affect voltage-operated calcium channels (Han et al., 1987; Benham and Tsien, 1988; Nelson et al., 1988).

Judging from responses to calcium in mesenteric resistance arteries that had been depleted of intracellular calcium, responses to noradrenaline and high potassium are not simply additive (Fig. 4.3). Half maximal contractile responses to the combination of noradrenaline and potassium could be obtained in the presence of extracellular calcium concentrations that were below the threshold needed to support any response to either noradrenaline or depolarisation alone. This synergism suggests an interaction of depolarisation and noradrenaline at a common component of the excitation-contraction coupling mechanism.

Other observations that suggest such an interaction include (i) the depolarising effect of noradrenaline in resistance-arterial smooth muscle as a consequence of increased influx of calcium ions (Cauvin and Van Breemen, 1985), and (ii) inhibition of contractile responses to noradrenaline in resistance arteries by hyperpolarising interventions such as activation of electrogenic sodium transport, opening of potassium channels and $\beta$-adrenergic stimulation (Mulvany et al., 1982; De Mey and Gray, 1985; Rinaldi and Bohr, 1989). 
One possible mechanism of interaction between the responses to depularisation and the catecholamine is that noradrenaline, through effects on a superficial intracellular calcium store, reduces the removal of calcium ions that enter the cell through voltage-operated channels (Saida and Van Breemen, 1984; Hurwitz, 1986). In the present study, noradrenaline, unlike high potassium, induced a single transient contractile response after the removal of extracellular calcium. This suggests the presence of a noradrenaline-sensitive intracellular calcium store (Saida and Van Breemen, 1984). Another possibility is that second messengers produced in response to the catecholamine increase the sensitivity of the contractile proteins to calcium (Adelstein and Sellers, 1987; Rasmussen et al., 1987; Sharma and Bhalla, 1988). This could explain the difference in calcium sensitivity between vessels stimulated with $\mathrm{K}-\mathrm{KRB}$ or noradrenaline but not the further increase in calcium sensitivity which is seen in the presence of both stimuli.

Furthermore, neither mechanism explains the susceptibility to dihydropyridines of resistance arterial contractile responses to noradrenaline. In previous studies in resistance arteries, dihydropyridine calcium antagonists were observed to reduce responses to calcium in the presence of noradrenaline with a higher or comparable potency than those in the presence of depolarising solution (Nyborg and Mulvany, 1984; Cauvin and Van Breemen, 1985; Julou-Schaeffer and Freslon, 1987). In the present study, nimodipine was ten times less potent in reducing responses to calcium in the presence of noradrenaline than in reducing those in depolarising solution. For comparison; this difference rises to a hundred to a thousand fold in large elastic arteries (Scriabine and Van den Kerckhoff, 1988; Chapter 3). In addition, the dihydropyridine agonist Bay-K8644 increased the calcium sensitivity of resistance arteries in the presence of either noradrenaline or high potassium. Assuming that dihydropyridines selectively interfere with voltage operated calcium channels, our observations suggest that noradrenaline and depolarisation interact at these channels.

We propose that noradrenaline can regulate the function of the same calcium channels that are operated upon by changes in membrane potential. In arterial smooth muscle, as in the myocardium (Hess et al., 1984; Bean et al., 1986), depolarisation could initially activate resting voltage-operated calcium channels. As the depolarisation is maintained, an equilibrium is established between activated and inactivated channels. High affinity binding of calcium antagonists to the latter (Hess et al., 1984; Bean et al., 1986) reduces the number of channels involved in the dynamic equilibrium between activated and inactivated channels. Previous observations (Högestätt and Andersson, 1984; Nyborg and Mulvany, 1984) of biphasic contractile responses to K-KRB in resistance arteries showed that the initial transient response is far less sensitive to dihydropyridine calcium antagonists than the subsequent sustained response. These contractile phases could reflect subsequent activation of T-type and L-type voltage-operated calcium channels (Hermsmeyer et al., 1989). They could also be a consequence of the activation of the suggested three state calcium-channel. Contractile responses, though obtained in a more physiological environment than direct electrophysiological measurements of currents through single calcium channels, are a relatively indirect measure of calcium channel activation. Despite this, we hy- 
pothesize that within this framework some of the effects of noradrenaline are compatible with the possibility that this catecholamine shifts the equilibrium towards the activated state of the voltage-operated calcium channel.

Activation of voltage operated calcium channels by noradrenaline could be brought about by direct $G$ protein activation (Brown and Birnbaumer, 1988; Rosenthal et al., 1988), channel phosphorylation through diacylglycerol and protein kinase- $C$ or channel activation by inositol triphosphate or its metabolites (Rasmussen et al., 1987; Benham and Tsien, 1988; Sharma and Bhalla, 1988; Zeng et al., 1989).

The hypothesis that noradrenaline affects the function of voltage-operated calcium channels is strengthened by the observed effects of nimodipine and Bay-K8644 on contractile responses to extracellular calcium. In depolarised resistance arteries, the dihydropyridine calcium antagonist decreased the sensitivity to calcium, while the dihydropyridine calcium "agonist" increased the sensitivity to this divalent cation. In resistance arteries exposed to noradrenaline, the sensitivity to calcium was higher than during depolarisation and it could be increased further, although only slightly, by Bay-K8644. In addition, higher concentrations of nimodipine were required to reduce calcium sensitivity in the presence of noradrenaline than in high potassium solution. We propose that upon exposure to noradrenaline a small fraction of the "voltage operated" calcium channels are activated and that most of these remain in the activated state at each point in time, therewith reducing the number of potential binding sites for dihydropyridines. Strong depolarising stimuli would recruit considerably more channels but, a large proportion of these channels would enter into the inactivated state after an initial transient. To judge from the synergism between noradrenaline and high potassium with respect to calcium sensitivity, the effects of noradrenaline on the equilibrium between activated and inactivated calcium channels persist in the depolarised cell membrane.

\section{Hypersensitivity to calcium in the SHR}

Resistance arteries of spontaneously hypertensive rats displayed hypersensitivity to calcium when exposed to noradrenaline but not when exposed to high potassium. Furthermore, responses to calcium in the presence of noradrenaline in resistance arteries of SHR consisted of both a tonic and a rhythmic component. Rhythmicity was absent in the presence of high potassium and was not observed in WKY resistance arteries exposed to either noradrenaline or potassium. This confirms earlier observations in mesenteric resistance arteries of both young and adult spontaneously hypertensive rats (Mulvany and Nyborg, $1980)$ and in isolated vascular smooth muscle cells of spontaneously hypertensive rats (Bolzon and Cheung, 1989).

Although the hypersensitivity to calcium in SHR resistance arteries was obvious in the presence of noradrenaline, but not high potassium, it could be abolished by nimodipine. This suggests a role for voltage-operated calcium channels. To judge from Fig. 4.3., most of the hypersensitivity to calcium can be attributed to the occurrence of rhythmicity in resistance arteries of the SHR. Although rhythmic contractile reactivity was normally absent in resistance 
arteries of WKY, it could be induced by Bay-K8644 (Fig. 4.5). This further strengthens the suggestion that voltage-operated calcium channels are involved in the rhythmic responses and hypersensitivity to calcium in resistance arteries. In addition, the latter observation with Bay-K8644 indicates that the anatomical requirements for rhythmic responses in a multicellular preparation, which include a "pacemaker" that sets the rhythm and intercellular communication (Berridge and Galione, 1988), are present in resistance arteries of both SHR and WKY.

The effect of nimodipine on contractile responses to calcium during stimulation with noradrenaline were more pronounced in SHR than in WKY vessels. Those of Bay-K8644 were more pronounced in WKY vessels than in SHR vessels. This suggests that voltage-operated channels play a role in the hypersensitivity to calcium during exposure of SHR resistance arteries to noradrenaline. If noradrenaline indeed modulates voltage-operated calcium channel function, as suggested above, then these effects suggest a stronger effect of noradrenaline on voltage-operated calcium channel function in SHR vessels. This could be in line with several recent reports describing increased phospholipase C activity in arterial smooth muscle of SHR (Karaki et al., 1988; Uehara et al., 1988). Alternatively, altered calcium activated potassium efflux (Jones et al., 1988) might participate as well. Rhythmic responses in the stroke prone SHR substrain have been attributed to potassium channels (Berridge and Galione, 1988).

In summary, this study confirms hypersensitivity to calcium and rhythmicity in resistance arteries of SHR exposed to noradrenaline. These alterations can be modified by nimodipine and Bay-K8644. We suggest that in resistance arterial smooth muscle, noradrenaline modulates the function of voltage-operated calcium channels. This modulation could differ quantitatively in resistance arteries of SHR when compared to WKY. 



\section{G-Proteins are Involved in Contractile Responses of Isolated Mesenteric Resistance Arteries to Agonists}

Harrie C.M. Boonen and Jo G.R. De Mey

Department of pharmacology, University of Limburg, Maastricht, the Netherlands.

(Naunyn Schmiedeberg's Arch. Pharmacol. 1990, 342; 462-468) 


\section{SUMMARY}

We evaluated whether GTP-binding regulatory proteins (G-proteins) are involved in responses of resistance arterial smooth muscle to contractile agonists. We therefore pretreated isolated sympathectomised mesenteric resistance arteries of the rat with pertussis toxin (PTX) and recorded their contractile responses to aluminium fluoride, endothelin, high potassium, phenylephrine, phorbol myristate acetate, serotonin and vasopressin. PTX reduced contractile responses to agonists with the following order of potency: phenylephrine $=$ serotonin $>$ vasopressin $=$ endothelin. The toxin reduced responses to phenylephrine in both the presence and absence of extracellular $\mathrm{Ca}^{2+}$. In $\mathrm{Ca}^{2+}$-depleted vessels that were exposed to phenylephrine, PTX virtually abolished responses to $\mathrm{Ca}^{2+}$ while hardly affecting responses to $\mathrm{Ca}^{2+}$ in the presence of endothelin. Also aluminium fluoride and phorbol myristate acetate induced contractions. These were dependent on extracellular $\mathrm{Ca}^{2+}$ and inhibited by felodipine. PTX reduced responses to aluminium fluoride but not those to phorbol myristate acetate. These data indicate that PTX sensitive G-proteins are involved in both influx of $\mathrm{Ca}^{2+}$ and release of intracellular $\mathrm{Ca}^{2+}$ following $\alpha_{1}$-adrenergic and serotonergic stimulation of resistance arteries. The role of G-proteins in stimulated $\mathrm{Ca}^{2+}$ influx could involve a direct effect on calcium channels although an indirect effect through protein kinase- $C$ can not be entirely excluded. The persistance of contractile responses to vasopressin and endothelin following PTX suggests that these agonists engage different pathways to induce contraction or have a higher efficacy in activating similar G-proteins.

\section{INTRODUCTION}

Alterations in the regulation of intracellular calcium in vascular smooth muscle could play a role in the pathogenesis of hypertension (Sharma and Bhalla, 1988; Dominiczak and Bohr, 1989; Hermsmeyer and Rusch, 1989). Calcium can enter the cytoplasm through channels in the plasma membrane or from the sarcoplasmic reticulum. The sarcolemmal calcium channels can be opened by different mechanisms such as changes in membrane potential, stretch and receptor activation (Laher and Bevan, 1989; Rüegg et al., 1989; Zelis and Moore, 1989). The electrophysiological characteristics of voltage operated calcium channels are well documented (for review see: Bean, 1989). The precise interaction between different receptors and calcium channels, however, is still unclear. There are indications that receptor activation can modulate the function of the same calcium channels that are affected by changes in membrane potential (Han et al., 1987; Benham and Tsien, 1988; Nelson et al., 1988; Boonen and De Mey, 1990). In this respect, G-proteins could be the transducers in the agonist induced elevation of intracellular calcium, either directly via a coupling to a sarcolemmal calcium channel or indirectly through the activation of phospholipase- $C$, which in turn will generate second messengers that can cause the release of intracellular calcium and open calciumchannels in the cell membrane.

Nichols et al., (1989) have shown in pithed rats that postjunctional $\alpha_{2}-$ adrenoceptors may be linked directly to calcium-channels through pertussis 
toxin (PTX) sensitive G-proteins, whereas $\alpha_{1}$-adrenoceptors may be linked to two distinct G-proteins. One of these would also be pertussis toxin-sensitive and directly coupled to calcium channels. The other would be pertussis toxininsensitive and involved in the release of intracellular calcium through activation of phospholipase-C and subsequent generation of inositol triphosphate. Recently, Rosenthal et al., (1988) and Zeng et al., (1989) reported the involvement of G-proteins in the activation of voltage operated calcium channels in vascular smooth muscle.

The involvement of G-proteins and second messengers in calcium influx and intracellular release of stored calcium in resistance-sized arterial smooth muscle after stimulation with an agonist is largely unknown. Therefore we evaluated the effects of pertussis toxin, a bacterial inactivator of certain subtypes of G-proteins, on contractile responses to different agonists and calcium in isolated mesenteric resistance-sized arteries of the rat. The pharmacological agonists that were used have been observed to increase the turnover of phosphatidylinositol in vascular smooth muscle (Minneman, 1988; Akbar et al., 1989; Huang et al., 1989; Kondo et al., 1989). We also evaluated the effects of aluminium fluoride, a direct activator of G-proteins (Zeng et al., 1989) and phorbol myristate acetate, a direct activator of protein kinase C (Nishizuka, 1986) to elucidate their involvement in excitation-contraction coupling in these vessels.

\section{MATERIAL AND METHODS}

\section{Agents and drugs}

During dissection and experimentation the vessel preparations were immersed in a Krebs-Ringer bicarbonate (KRB) solution ( $\mathrm{pH} 7.4$ ) with the following composition $(\mathrm{mM})$ : $\mathrm{NaCl} 118.3 ; \mathrm{KCl} 4.7 ; \mathrm{CaCl}_{2}, 2.5 ; \mathrm{MgSO}_{4} .7 \mathrm{H}_{2} \mathrm{O}, 1.2$; $\mathrm{KH}_{2} \mathrm{PO}_{4}, 1.2 ; \mathrm{NaHCO}_{3}, 25.0$ and glucose, 11.1. High potassium solution (K-KRB) had a similar composition as $\mathrm{KRB}$ except that all the $\mathrm{NaCl}$ was replaced by an equimolar amount of $\mathrm{KCl}$. Ca ${ }^{2+}$-free $\mathrm{KRB}$ was obtained by omitting $\mathrm{CaCl}_{2}$ from normal KRB and adding $0.3 \mathrm{mM}$ EGTA. The drugs used included 6-hydroxydopamine, l-phenylephrine hydrochloride, ethyleneglycol-bis-( $\beta$-amino-ethyl ether) $N, N^{\prime}$-tetra acetic acid (EGTA), sodium fluoride and phorbol 12-myristate 13-acetate (Sigma, St.Louis Missouri, USA). Serotonin creatinine sulfate monohydrate and pertussis toxin were obtained from Janssen Chimica (Beerse, Belgium). Porcine endothelin-1 was obtained from Peninsula (Belmont, California, USA) and vasopressin from Sandoz AG (Basel, Switzerland). Aluminium chloride (p.a) was obtained from Merck (Darmstadt, FRG). Felodipine was kindly supplied by Dr. M. Nordlander (Hässle, Mölndal, Sweden). A stock solution was made by dissolving felodipine in polyethylene glycol $(10 \mathrm{mM})$. From this solution further dilutions were made in bidistilled water. 


\section{Preparation of vessel segments}

Adult male Wistar Kyoto rats were killed by cervical dislocation and exsanguination. A loop of the small intestine was isolated from the rats and immersed in KRB solution. Third to fourth order side branches of the superior mesenteric artery were then dissected from the mesentery. The adrenergic nerve endings in the vessels were destroyed by incubating the vessel segments for 10 minutes at $37^{\circ} \mathrm{C}$ in a bicarbonate free Krebs-Ringer solution ( $\mathrm{pH} 4.0$ ) that contained $300 \mu \mathrm{g} / \mathrm{ml}$ 6-hydroxydopamine (Aprigliano and Hermsmeyer, 1977). The vessel segments (approximately $2 \mathrm{~mm}$ in length) were then threaded on two stainless steel wires $(\varnothing 40 \mu \mathrm{m})$ and mounted horizontally as ring segments in an organ chamber between an isometric force transducer (Kistler Morse DSC 6, Seattle USA) and a displacement device (Mulvany and Halpern, 1977). The solution in the myograph was maintained at $37^{\circ} \mathrm{C}$ and aerated with $95 \% \quad \mathrm{O}_{2}$ and $5 \%$ $\mathrm{CO}_{2}$. Prior to experimentation the vessels were stretched to their individual optimal lumen diameter for force development as was previously described (De Mey et al., 1987). Two vessels could be mounted in parallel in one organ chamber.

\section{Experiments with pertussis toxin}

Prior to mounting and experimentation, the vessels were incubated for $90 \mathrm{~min}$. at $37^{\circ} \mathrm{C}$ in $1 \mathrm{ml} \mathrm{KRB}$ containing $0.0,0.2,1.0$ or $5.0 \mu \mathrm{g} / \mathrm{ml}$ pertussis toxin. In the first series of experiments concentration-response curves were constructed for endothelin (0.1 nM - $30 \mathrm{nM})$, phenylephrine $(0.1 \mu \mathrm{M}-12.5 \mu \mathrm{M})$, serotonin $(0.1 \mu \mathrm{M}-12.5 \mu \mathrm{M})$ and vasopressin $(0.1 \mathrm{nM}-30 \mathrm{nM})$, in control and pertussis toxin-pretreated preparations. In the second series of experiments calcium-concentration response curves were constructed in the presence of 10 $\mu \mathrm{M}$ phenylephrine or $30 \mathrm{nM}$ endothelin in pertussis toxin-pretreated and control preparations. During 3 minutes, the vessels were incubated in $\mathrm{Ca}^{2+}$-free KRB. Then, either $10 \mu \mathrm{M}$ phenylephrine or $30 \mathrm{nM}$ endothelin was administered in the bath. This resulted in a transient contractile response. A second application of the agonists in $\mathrm{Ca}^{2+}$-free solution, did no longer induce contraction indicating depletion of the respective agonist-sensitive intracellular calcium stores (Van Breemen and Saida, 1989). Calcium concentration response curves $(25 \mu \mathrm{M}-2.5$ $\mathrm{mM}$ ) were then constructed in the continuous presence of either $10 \mu \mathrm{M}$ phenylephrine or $30 \mathrm{nM}$ endothelin. Calcium concentration response curves could not be obtained in the presence of serotonin or vasopressin because of the tachyphylactic nature of the contractile responses to these agonists.

\section{Experiments with aluminium fluoride and phorbol myristate acetate}

In order to directly activate G-proteins, vessels were incubated for 20 minutes in the presence of a combination of $5 \mathrm{mM}$ sodium fluoride $(\mathrm{NaF})$ and $32 \mu \mathrm{M}$ aluminium chloride $\left(\mathrm{AlCl}_{3}\right)$. $\mathrm{AlF}_{4}{ }^{\prime}$ which has a similar structure as $\mathrm{PO}_{4}{ }^{3 .}$, 
can interact with the $\alpha$-subunit of the G-protein. This results in activation of the G-protein (Zeng et al., 1989). These experiments were performed in the presence or absence of $2.5 \mathrm{mM}$ calcium.

Phorbol myristate acetate has been shown to increase the activity of protein kinase-C in a variety of cell types including vascular smooth muscle (Nishizuka, 1986; Fish et al., 1988). To evaluate the role of protein kinase-C in the activation of $\mathrm{G}$-proteins, we induced contractile responses to $1 \mu \mathrm{M}$ phorbol myristate acetate in control vessels and in vessels that had been pretreated with $1 \mu \mathrm{g} / \mathrm{ml}$ pertussis toxin.

\section{Statistics}

Differences in contractile responses to the agonists between control and pertussis toxin-pretreated vessels were evaluated using an unpaired t-test. In the case of multiple comparisons, one way ANOVA was followed by a modified $\mathrm{t}$-test according to the method described by Bonferroni (Wallenstein et al., 1980). In most cases, $\mathrm{P}<0.05$ was accepted to denote statistical significance of differences except for multiple comparisons $(P=0.05 / \mathrm{m}$, in which $\mathrm{m}$ is the number of comparisons). Contractile responses are expressed as increases in wall tension (increases in force divided by twice the vessel segment length).

\section{RESULTS}

\section{Mechanical properties}

The optimal lumen diameter of the isolated sympathectomised mesenteric resistance vessels averaged $214 \pm 4 \mu \mathrm{m}(n=49)$. This did not differ between control and pertussis toxin-pretreated vessels. Contractile responses induced by exposing the vessels to $125 \mathrm{mM} \mathrm{K-KRB}$, consisted of an initial transient $(2.58 \pm 0.20 \mathrm{mN} / \mathrm{mm}, \mathrm{n}=18)$ and a subsequent sustained phase $(2.35 \pm$ $0.15 \mathrm{mN} / \mathrm{mm}, \mathrm{n}=18)$.

\section{Effects of pertussis toxin on contractile responses to agonists and calcium}

Pretreatment of vessels with pertussis toxin had no significant effect on the transient contractile response to potassium. It averaged $2.92 \pm 0.25$ $\mathrm{mN} / \mathrm{mm}(\mathrm{n}=7) ; 2.39 \pm 0.17 \mathrm{mN} / \mathrm{mm}(\mathrm{n}=18)$ and $2.34 \pm 0.38 \mathrm{mN} / \mathrm{mm}(\mathrm{n}=7)$ in vessels that had been pretreated with $0.2 ; 1.0$; and $5.0 \mu \mathrm{g} / \mathrm{ml}$ pertussis toxin, respectively. These responses were used as a reference to which all contractile responses induced by the agonists were related. Unlike the transient contractile response to $125 \mathrm{mM}$ potassium, the sustained response was reduced following pertussis toxin. It averaged $2.67 \pm 0.23 \mathrm{mN} / \mathrm{mm}(\mathrm{n}=7) ; 1.31 \pm 0.16 \mathrm{mN} / \mathrm{mm}$ $(n=18)(P<0.05$ vs. control, one-way ANOVA) and $1.60 \pm 0.31 \mathrm{mN} / \mathrm{mm}(n=7)$ 


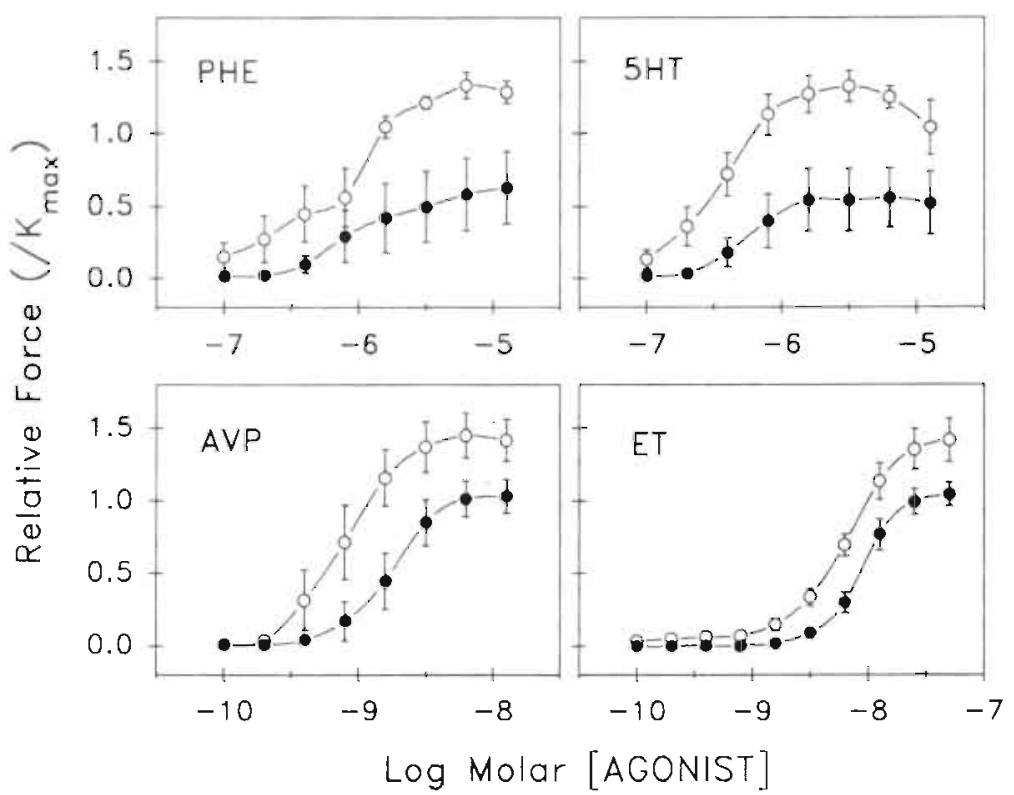

Figure 5.1. Concentration response curves in mesenteric resistance arteries for phenylephrine (PHE, upper left), serotonin (5HT, upper right), vasopressin (AVP, lower left) and endothelin (ET, lower right) in the presence of $2.5 \mathrm{mM} \mathrm{Ca}^{2 *}$ in control vessels (open circles) or in vessels pretreated with $1 \mu \mathrm{g} / \mathrm{ml}$ pertussis toxin (solid circles). Shown are mean values \pm SEM as fraction of the maximal transient contractile responses to $125 \mathrm{mM}$ K-KRB.

$(P<0.05$ vs. control, one-way ANOVA) in vessels pretreated with $0.2 ; 1.0$; and $5.0 \mu \mathrm{g} / \mathrm{ml}$ pertussis toxin, respectively. Maximal contractile responses and sensitivities of mesenteric resistance arteries to phenylephrine, serotonin, vasopressin and endothelin are summarised in Table 5.1. Treatment of vessels with 1 $\mu \mathrm{g} / \mathrm{ml}$ pertussis toxin significantly decreased the maximal responses and sensitivities to the different agonists with the following order of potency : phenylephrine $=$ serotonin $>$ vasopressin $=$ endothelin (Table 5.1, Fig. 5.1). Effects of pertussis toxin on responses to phenylephrine were more marked after pretreatment of the vessels with 1.0 than with $0.2 \mu \mathrm{g} / \mathrm{ml}$ of the toxin. Effects of 1.0 and $5.0 \mu \mathrm{g} / \mathrm{ml}$ pertussis toxin on responses to the $\alpha_{1}$-adrenoceptor agonist were comparable.

In the absence of extracellular calcium, $30 \mathrm{nM}$ endothelin, $10 \mu \mathrm{M}$ phenylephrine, $30 \mathrm{nM}$ vasopressin but not $125 \mathrm{mM} \mathrm{K}-\mathrm{KRB}$, induced a transient contraction. These contractions, which are due to the release of intracellularly stored calcium (Van Breemen and Saida, 1989), averaged $50 \pm 4 \%(n=7), 48 \pm 4 \%$ $(n=7)$, and $66 \pm 3 \%(n=7)$ of the response to $125 \mathrm{mM} \mathrm{K-KRB}$ in the presence of 


\section{G-PROTEIN INVOLVEMENT IN CONTRACTION OF SMALL ARTERIES}

Table 5.1. Effects of $1 \mu \mathrm{g} / \mathrm{ml}$ pertussis toxin on maximal contractile responses and sensitivities to different agonists in sympathectomised mesenteric resistance arteries of WKY.

\begin{tabular}{|c|c|c|c|}
\hline $\operatorname{Max}(/ K-K R B)$ & control & PTX & $n$ \\
\hline $\mathrm{K}-\mathrm{KRB}_{s}$ & $0.87 \pm 0.05$ & $0.64 \pm 0.07^{\circ}$ & 12 \\
\hline PHE $10 \mu \mathrm{M}$ & $1.28 \pm 0.04$ & $0.68 \pm 0.09^{\mathrm{a}}$ & 25 \\
\hline $5 \mathrm{HT} 10 \mu \mathrm{M}$ & $1.36 \pm 0.12$ & $0.59 \pm 0.21$ & 6 \\
\hline AVP $30 \mathrm{nM}$ & $1.37 \pm 0.08$ & $1.12 \pm 0.07^{a}$ & 12 \\
\hline $\mathrm{ET} 30 \mathrm{nM}$ & $1.42 \pm 0.15$ & $1.10 \pm 0.08^{\circ}$ & 6 \\
\hline$A L F_{i}$ & $0.81 \pm 0.15$ & $0.07 \pm 0.04$ & 6 \\
\hline PMA & $0.95 \pm 0.13$ & $0.65 \pm 0.11$ & 6 \\
\hline \multicolumn{4}{|c|}{ Sensitivity (-Log Molar $\left[E_{50}\right]$ ) } \\
\hline PHE & $6.12 \pm 0.12$ & $5.78 \pm 0.13^{a}$ & 12 \\
\hline $5 \mathrm{HT}$ & $6.52 \pm 0.10$ & $6.08 \pm 0.14^{2}$ & 6 \\
\hline AVP & $9.11 \pm 0.11$ & $8.75 \pm 0.09^{a}$ & 6 \\
\hline ET & $8.22 \pm 0.06$ & $8.05 \pm 0.06^{\circ}$ & 6 \\
\hline
\end{tabular}

Top: Maximal sustained contractile responses to $125 \mathrm{mM}$ potassium (K-KRB), $10 \mu \mathrm{M}$ phenylephrine (PHE), $10 \mu \mathrm{M}$ serotonin ( $5 \mathrm{HT}$ ), $30 \mathrm{nM}$ vasopressin (AVP) $30 \mathrm{nM}$ endothelin (ET), AlF ${ }_{4}(5 \mathrm{mM} \mathrm{NaF}$ and $32 \mu \mathrm{M} \mathrm{AlCl}$ ) and $1 \mu \mathrm{M}$ phorbol myristate acetate (PMA) are shown as fraction of the maximal transient contraction evoked by $125 \mathrm{mM} \mathrm{K}$ $\mathrm{KRB}$ for control vessels and vessels pretreated with $1 \mu \mathrm{g} / \mathrm{ml}$ pertussis toxin during 90 min. Maximal transient responses to $125 \mathrm{mM} \mathrm{K-KRB}$ averaged $2.58 \pm 0.20$ and $2.39 \pm 0.17$ $\mathrm{mN} / \mathrm{mm}$ in control and pertussis toxin pretreated vessels, respectively. Bottom: $\mathrm{pD} 2$ values (- Log Molar $\left[E C_{\mathrm{s}}\right]$ ) for the agonists with or without pertussis toxin pretreatment are shown as mean \pm SEM. ": the difference from control is statistically significant. $n$ represents the number of observations (one-way ANOVA).

2,5 $\mathrm{mM}$ calcium, for endothelin, phenylephrine and vasopressin, respectively. In vessels that had been pretreated with $1 \mu \mathrm{g} / \mathrm{ml}$ pertussis toxin, responses to phenylephrine in calcium-free solution were strongly reduced $(8.9 \pm 3.1 \%$ of $\mathrm{K}$ KRB $125 \mathrm{mM}$ maximum)(Fig. 5.2). However, those to endothelin $(38 \pm 17 \%$ of K-KRB $125 \mathrm{mM}$ maximum) and vasopressin (56 $\pm 6 \%$ )(Fig. 5.2) were not significantly affected. Readministration of calcium to control vessels stimulated with $10 \mu \mathrm{M}$ phenylephrine or $30 \mathrm{nM}$ endothelin, resulted in concentration dependent increases in wall tension. Vessels pretreated with $1 \mu \mathrm{g} / \mathrm{ml}$ pertussis toxin failed to respond to increasing concentrations of calcium in the presence of $10 \mu \mathrm{M}$ phenylephrine but not in the presence of $30 \mathrm{nM}$ endothelin (Fig. 5.3). 

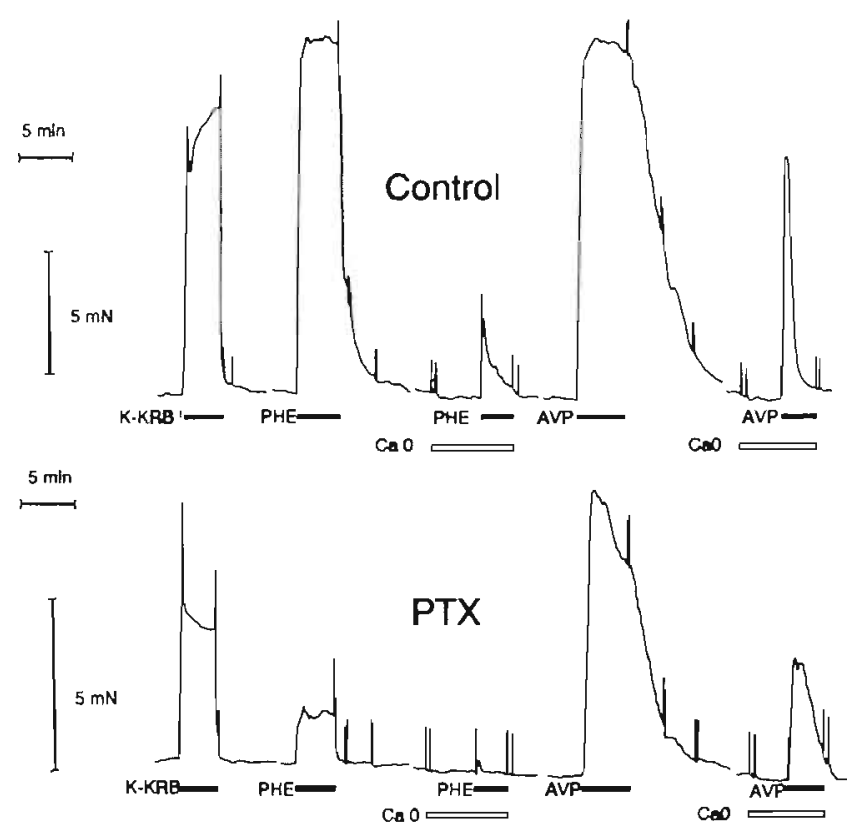

Figure 5.2. Redrawing of typical tracings of contractile responses to $125 \mathrm{mM}$ potassium (K-KRB), $10 \mu \mathrm{M}$ phenylephrine (PHE), or $30 \mathrm{nM}$ vasopressin (AVP) in isolated sympathectomised mesenteric resistance arteries. The upper tracing is of a control vessel, the lower of a vessel that was pretreated with $1 \mu \mathrm{g} / \mathrm{ml}$ pertussis toxin (PTX) during $90 \mathrm{~min}$. Responses to phenylephrine and vasopressin were repeated in the absence of extracellular calcium (Ca 0) (indicated by the open bars).

\section{Effects of aluminium fluoride and phorbol myristate acetate}

Sodium fluoride $(5 \mathrm{mM})$ induced a small contractile response in the presence of $2.5 \mathrm{mM}$ calcium. Addition of $32 \mu \mathrm{M}$ aluminium chloride had a strong potentiating effect on the contractions induced by $5 \mathrm{mM}$ sodium fluoride (Fig. 5.4). The contractile response to aluminium fluoride reached $2.32 \pm$ $0.43 \mathrm{mN} / \mathrm{mm}(\mathrm{n}=7)$, which is $81 \pm 15 \%$ of the maximal contractile response to $125 \mathrm{mM} \mathrm{K-KRB}$. These contractions were readily abolished in the absence of extracellular calcium or in the presence of the dihydropyridine calcium antagonist felodipine. Pertussis toxin significantly reduced contractile responses to aluminium fluoride. After pretreatment with $0.2,1.0$, or $5.0 \mu \mathrm{g} / \mathrm{ml}$ pertussis toxin, they averaged $0.49 \pm 0.29(n=7) ; 0.20 \pm 0.08 \quad(n=7)$ and $0.27 \pm 0.08$ $\mathrm{mN} / \mathrm{mm}(\mathrm{n}=7)$, respectively.

Phorbol myristate acetate $(1 \mu \mathrm{M})$ induced a slow contractile response. These responses averaged $1.85 \pm 0.32 \mathrm{mN} / \mathrm{mm}(95 \pm 13 \%$ of the maximal response to $125 \mathrm{mM} \mathrm{K-KRB})(\mathrm{n}=6)$. Pertussis toxin pretreatment did not affect contractile responses to $1 \mu \mathrm{M}$ phorbol myristate acetate $(1.71 \pm 0.34 \mathrm{mN} / \mathrm{mm}$; 


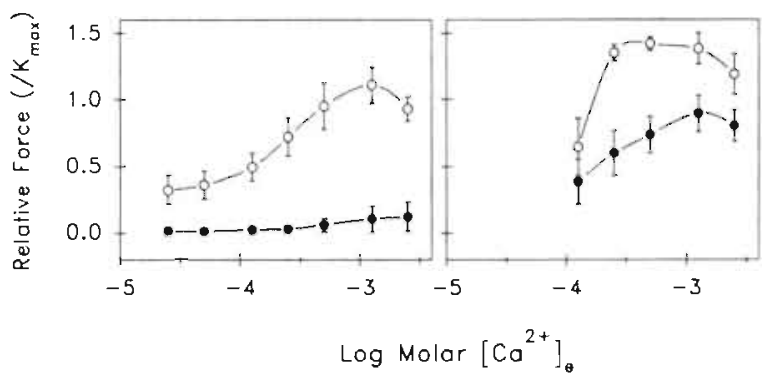

Figure 5.3. Concentration response curves to calcium, in calcium depleted vessels, in the continuous presence of $10 \mu \mathrm{M}$ phenylephrine (PHE, left) or $30 \mathrm{nM}$ endothelin (ET, right), in control vessels (open circles) and in vessels pretreated with $1 \mu \mathrm{g} / \mathrm{ml}$ pertussis toxin (solid circles). Shown are mean values \pm SEM as force development relative to the transient contractile responses to $125 \mathrm{mM} \mathrm{K-KRB}$.

Figure 5.4. Typical tracings of contractile responses to the combination of 5 $\mathrm{mM}$ sodium fluoride and $32 \mu \mathrm{M}$ aluminium chloride $\left(\mathrm{AlF}_{4}\right)$ in a control vessel (upper tracing and left calibration mark) and in a vessel pretreated with $1 \mu \mathrm{g} / \mathrm{ml}$ pertussis toxin during 90 min.. (lower tracing, right calibration mark). In the absence of extracellular calcium (Ca 0 , open bar), aluminium fluoride did not induce a contractile response. A contraction developed only upon the readministration of $2.5 \mathrm{mM}$

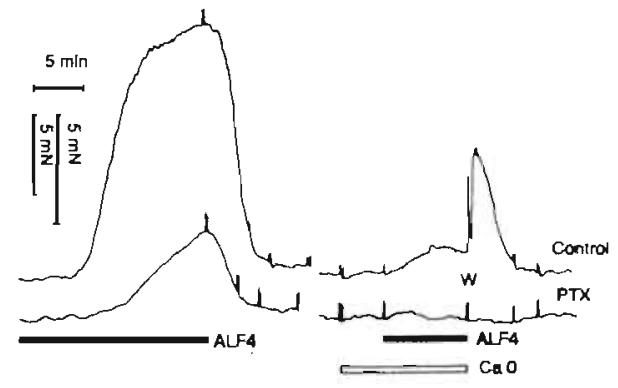
calcium $(W)$.

$65 \pm 11 \%$ of the $\mathrm{K}-\mathrm{KRB}$ maximum)( $\mathrm{n}=6)$. However, contractile responses to phorbol myristate acetate were strongly reduced by the dihydropyridine calcium antagonist felodipine (the $\mathrm{plC}_{50}$ for felodipine averaged $-9.40 \pm 0.49$ $(n=10))$, and by the removal of extracellular calcium.

\section{DISCUSSION}

We evaluated whether G-proteins are involved in contractile responses of isolated mesenteric resistance-sized arteries to agonists. Therefore, we recorded effects of pertussis toxin, which irreversibly inactivates certain G-proteins (Murayama and Ui, 1983; Ribeiro-Neto and Rodbell, 1989), and aluminium fluoride which directly activates G-proteins (Zeng et al., 1989), on contractile responses to diffe- 
rent agonists and calcium. Taken together the results suggest that pertussis toxin-sensitive G-proteins are involved in influx of calcium as well as release of intrac(llularly stored calcium during $\alpha_{1}$-adrenergic and serotonergic activation. We suggest that in mesenteric resistance arteries of the rat, pertussis toxin sensitive $G$-proteins couple $\alpha_{1}$-adrenoceptors to phospholipase- $C$ or directly to plasmalemmal calcium channels. Upon receptor stimulation, activation of these $G$-protcins can directly or indirectly affect calcium channels in the cell membrane.

\section{Effects of pertussis toxin on agonist induced contractions}

While pertussis toxin did not affect the transient contractile responses to potassium, pretreatment of the vessels with the toxin reduced responses to the pharmacological agonists and the sustained contractile response to potassium. Responsi's to $\alpha_{1}$-adrenergic and serotonergic stimulation were affected to a larger extent than responses to vasopressin or endothelin. Phenylephrine and serotonin have been shown to stimulate calcium influx and intracellular calcium release by increasing the phosphatidylinositol turnover in vascular smooth muscle (Minneman, 1988; Akbar et al., 1989). Our indirect observations thus suggest that in the case of phenylephrine and serotonin, pertussis toxin-sensitive G-proteins are involved in the agonist induced activation of phosphatidylinositol turnover in mesenteric resistance arteries. Also vasopressin can stimulate calcium influx and intracellular release of calcium by increasing phosphatidylinositol turnover (Grillone et al., 1988; Kondo et al., 1989). However, contractile responses to vasopressin were less affected by pertussis toxin than those to phenylephrine or serotonin. In some, but not all reports (Huang et al., 1989; Mitsuhashi et al., 1989; Ohlstein et al., 1989), also endothelin has been shown to increase phosphatidylinositol turnover. Yet, also contractile responses to this peptide were hardly affected by pertussis toxin. This discrepancy in effects of pertussis toxin on contractile responses to different agonists may indicate that vasopressin and endothelin engage different pathways to induce a cellular response, or that vasopressin and endothelin have a greater efficacy than phenylephrine and serotonin in activating similar G-proteins. A comparable suggestion was made by Liebau et al., (1989), who found that inhibition of $\alpha_{1}$-adrenoceptor mediated contractions by pertussis toxin, was dependent on the agonist intrinsic efficacy. Thus agonist-receptor complexes could have different potencies in activating one or more G-proteins. The inhibitory effect of pertussis toxin treatment on sustained contractile responses to potassium could indicate that depolarisation of sympathectomised vessels does not only lead to a direct stimulation of the contractile apparatus through opening of voltage dependent calcium channels in the sarcolemma, but also through release of neuropeptides. (Kawasaki et al, 1988). An alternative explanation could be that pertussis toxin-sensitive G-proteins are activated as a consequence of the increased calcium-influx stimulated by depolarisation. This, however, remains to be elucidated. 


\section{Effects of pertussis toxin on calcium mobilising pathways}

To separate the components of contractile responses that are due to influx of calcium and those that are due to the release of intracellular calcium, concentration response curves for calcium were constructed in the presence of $10 \mu \mathrm{M}$ phenylephrine or $30 \mathrm{nM}$ endothelin. As can be seen in Figure 5.2., vasopressin and phenylephrine evoked contractions in vessels that were incubated in $\mathrm{Ca}^{2+}$-free solution, indicating that they can induce the release of intracellularly stored calcium. Pretreatment with pertussis toxin led to an almost complete obliteration of the transient response to phenylephrine. However, contractile responses to vasopressin and endothelin in calcium free medium were not significantly affected after pertussis toxin treatment. In the continuous presence of phenylephrine or endothelin, readministration of calcium in calcium depleted preparations caused tonic increases in wall tension. These most likely result from influx of calcium from the extracellular medium. Contractile responses to calcium in the presence of phenylephrine were abolished by pertussis toxin while those to endothelin were affected to a much lesser extent. These and the above mentioned observations suggest that in mesenteric resistance arteries, pertussis toxin sensitive G-proteins are involved in stimulation of both release of intracellular calcium and influx of calcium following $\alpha_{1}$-adrenoceptor activation. Assuming that $\alpha_{1}$-adrenergic stimulation leads to activation of phospholipase-C (Minneman, 1988), one can conclude that pertussis toxin-sensitive $G$ proteins couple $\alpha_{1}$-adrenoceptors to phospholipase-C. It can not be excluded, however, that pertussis toxin-sensitive G-proteins could also directly affect calcium channels in the plasma membrane (Brown and Birnbaumer, 1988; Rosenthal et al., 1988; Zeng et al., 1989). Nevertheless, our observations are in contrast with those of Nichols et al., (1989), who suggested that $\alpha_{1}$-adrenoceptors are coupled to pertussis toxin-sensitive and pertussis toxin-insensitive G-proteins, the former of which couple the adrenoceptor directly to a calcium channel and the latter coupling the adrenoceptor to phospholipase-C, being responsible for intracellular calcium release.

\section{Effects of direct activation of G-proteins or protein kinase-C}

To assess whether G-protein activation directly modulates calcium channel function, we stimulated G-proteins with aluminium fluoride (Zeng et al., 1989). Aluminium fluoride induced slow concentration dependent sustained contractions that were strictly dependent on the presence of extracellular calcium. The contractions to aluminium fluoride were abolished by pertussis toxin. Thus, pertussis toxin-sensitive G-proteins were involved in the influx of calcium following activation with aluminium fluoride.

Also phorbol myristate acetate induced a sustained contraction. These contractions could be inhibited by the removal of calcium from the extracellular medium and by the dihydropyridine calcium antagonist felodipine. These observations suggest that contractions induced by phorbol myristate acetate depend primarily on the influx of calcium. Pertussis toxin pretreatment did not 


\section{CHAPTER 5}

affect contractile respunses to phorbol myristate acetate indicating that in the case of agonist-induced contractions, pertussis toxin-sensitive G-proteins were not activated as a result of stimulated protein kinase-C or increased calcium influx.

It can be concluded that pertussis toxin sensitive G-proteins are involved in $\alpha_{1}$-adrenergic and serotonergic stimulation of mesenteric resistance arteries. They either couple $\alpha_{1}$-adrenoceptors and serotonergic receptors directly to calcium influx or to phospholipase- $C$ which in turn generates second messengers that can i) increase the open probability of sarcolemmal calcium channels and ii) induce intracellular calcium release. Contractions induced by vasopressin and endothelin were affected less by pertussis toxin than those to phenylephrine or serotonin. This could be due to (i) the engagement of different mechanisms that lead to contraction or (ii) to a higher efficacy with which these agonist activate similar G-proteins. 


\section{Effects of a Phorbol Ester and Staurosporine on Electro- and Pharmacomechanical Coupling in a Resistance Artery}

Harrie C.M. Boonen and Jo G.R. De Mey

Department of pharmacology, University of Limburg, Maastricht, the Netherlands.

(Eur. J. Pharmacol. 1991, 202: 25-32.) 


\section{SUMMARY}

We examined the role of protein kinase-C in contractile responses of small arteries of the rat by stimulating and inhibiting protein kinase-C with phorbol myristate acetate and staurosporine, respectively. The experiments were performed in isolated mesenteric resistance arteries that had been sympathectomised and mounted for recurding of isometric force development. Phorbol myristate acetate i) increased from below 3 $\mathrm{nM}$ the sensitivity for the contractile effect of potassium, but not noradrenaline or BayK8644, ii) increased from $30 \mathrm{nM}$ the sensitivity of depolarised vessels for extracellular calcium and iii) induced from $30 \mathrm{nM}$ a contractile effect that depended on the presence of extracellular calcium and that was reduced by the calcium antagonist felodipine. Both the phorbol ester and staurosporine did not affect contractile responses to caffeine in calcium free solution. $10 \mathrm{nM}$ staurosporine reduced resistance arterial responses to potassium but not noradrenaline. These results are in agreement with direct observations by others that suggest a role for protein kinase-C in the activation of voltage operated calcium channels. Protein kinase- $C$ could participate in this way in electro-mechanical coupling in resistance arterial smooth muscle and when strongly activated, sensitise the contractile apparatus for calcium.

\section{INTRODUCTION}

The tone and structure of small arteries govern peripheral vascular resistance and local blood flow (Mellander, 1989; Folkow, 1990; Nelson et al., 1990). Tone is controlled by myogenic mechanisms and by neurohumoral and paracrine vasoactive substances. These modulate the concentration of free calcium ions in the smooth muscle cells (Carafoli, 1987; Nelson et al., 1990). The cytosolic free calcium concentration is regulated by influx of calcium ions into the cell, their release and sequestration by intracellular stores and their extrusion from the cell across the cell membrane (Carafoli, 1987; Van Breemen and Saida, 1989). Calcium influx depends primarily on the function of calcium channels in the sarcolemma. The function of these channels can be modulated by both changes in membrane potential and vasuactive agonists (Nelson et al., 1988, 1990; Somlyo and Himpens, 1989; Boonen and De Mey, 1490b).

Vasoconstrictor substances affect the function of voltage-operated calcium channels in different cell types. This is brought about either directly through the activation of GTP-binding regulatory proteins or indirectly via activation of phospholipase-C (Zeng et al., 1989; Boonen and De Mey, 1990a; Nelson et al, 1990). Activation of phospholipase-C leads to the generation of inositol-triphosphate and diacylglycerol. The former stimulates the release of calcium ions from intracellular stores. Diacylglycerol, on the other hand, activates protein kinase-C (Berridge, 1986; Nishizuka, 1986). This kinase can modify several processes. It can increase the sensitivity of the contractile apparatus to calcium (Adelstein and Sellers, 1987; Rasmussen et al, 1987; Khalil and Van Breemen, 1988; Drenth et al., 1989), decrease the activity of phospholipase- $C$ (Yamatani et al., 1990), activate sodium- 
hydrogen exchange (Boscoboinik et al., 1990), desensitise various membrane receptors (Huganir and Greengard, 1987; Raymond et al., 1990) and affect the function of ion channels in the cell membrane (Litten et al., 1987; Fabbro et al., 1988). Direct observations (Gleason and Flaim, 1986; Fish et al., 1988) have shown that phorbol esters increase calcium influx through voltage operated calcium channels in vascular smooth muscle cells.

The exact role of protein kinase-C in the muscular lining of blood vesisels that are small enough to contribute to the control of peripheral vascular resistance and local blood flow is, however, largely unknown. In view of several pharmacological peculiarities of the excitation-contraction coupling in resistance arterial smooth muscle (Mulvany et al., 1982; Cauvin et al., 1985), this can not be directly extrapolated from observations in large arteries or non-vascular smooth muscle preparations. Moreover, in the resistance part of the circulation not only pharmaco-mechanical coupling but also electro-mechanical coupling is functionally relevant (Nelson et al., 1990). Contractile responses to depolarisation participate in the effects of vasoconstrictor agents and underlie myogenic arteriolar responses and autoregulation of blood flow (Mulvany et al., 1982; Mellander, 1989; Nelson et al., 1990).

Based on the observations in vascular smooth muscle cells which suggest that protein kinase- $C$ can affect voltage operated calcium channels (Gleason and Flaim, 1986; Fish et al., 1988), we evaluated whether protein kinase- $C$ is involved in mechanical responses of isolated mesenteric resistance arteries to depolarisation and pharmacological stimulation. Since the small size of the preparations hinders biochemical analyses, we selected a pharmacological approach. We recorded effects on resistance arterial contractile responses of phorbol myristate acetate and staurosporine, an activator and an inhibitor of protein kinase-C, respectively (Nishizuka, 1986; Rüegg and Burgess, 1989).

\section{MATERIAL AND METHODS}

\section{Preparation of vessels}

Adult Wistar Kyoto rats were killed by a sharp blow on the head and exsanguination. Resistance sized arteries (diameter approximately $200 \mu \mathrm{m}$ ) were then dissected from the mesentery. Since various pharmacological interventions, as well as depolarisation, affect not only smooth muscle cells but also sympathetic nerves in the vascular wall (Vanhoutte et al., 1981), the vessels were acutely sympathectomised. This was achieved by incubating the vessels for 10 minutes at $37^{\circ} \mathrm{C}$ in a bicarbonate free Krebs Ringer solution ( $\mathrm{pH} 4.0$ ) that contained $300 \mu \mathrm{g} / \mathrm{ml}$ 6-hydroxydopamine (Aprigliano and Hermsmeyer, 1977). The vessels were then mounted as ring segments between an isometric force transducer (Kistler Morse DSC 6, Seattle USA) and a displacement device (Mulvany and Halpern, 1977). The solution in the myograph was maintained at $37^{\circ} \mathrm{C}$ and aerated with $95 \% \mathrm{O}_{2}$ and $5 \% \mathrm{CO}_{2}$. Prior to experimentation the vessels were 
stretched to their individual optimal lumen diameter for force development (De-Mey et al., 1987). The current output of the strain gauge was amplified and digitised on line. The digitised force output was displayed and stored on an Olivetti PC/AT M28 and analysed using a software package prepared by the Instrumental Development Department (University of Limburg, Maastricht, The Netherlands). During dissection, mounting and experimentation the vessels were immersed in a Krebs Ringer bicarbonate (KRB) solution ( $\mathrm{pH} 7.4$ ) with the following composition $(\mathrm{mM})$ : $\mathrm{NaCl}, 118.3 ; \mathrm{KCl}, 4.7 ; \mathrm{CaCl}_{2}, 2.5 ; \mathrm{MgSO}_{4} .7 \mathrm{H}_{2} \mathrm{O}$, 1.2; $\mathrm{KH}_{2} \mathrm{PO}_{4}, 1.2 ; \mathrm{NaHCO}_{3}, 25.0$ and glucose, 11.1. The high potassium solution (K-KRB) had a composition similar to that of $\mathrm{KRB}$ except that all the $\mathrm{NaCl}$ had been replaced by an equimolar amount of $\mathrm{KCl}$. Solutions with potassium concentrations ranging from 5.9 to $125 \mathrm{mM}$ were obtained by mixing appropriate volumes of $\mathrm{KRB}$ and $\mathrm{K}-\mathrm{KRB}$. For $\mathrm{Ca}^{2+}$-free $\mathrm{KRB}, \mathrm{CaCl}_{2}$ was omitted from normal KRB and $0.3 \mathrm{mM}$ EGTA was added. The drugs used included 6-hydroxydopamine, caffeine, ethylene glycol bis ( $\beta$-aminoethyl-ether)- $\mathrm{N}-\mathrm{N}^{\prime}$-tetraacetic acid (EGTA), l-arterenol bitartrate (noradrenaline), phorbol 12-myristate 13acetate and dl-propranolol HCl (Sigma, St.Louis MO, USA). Stock solutions of phorbol myristate acetate $(1 \mathrm{mM})$ were prepared by dissolving phorbol myristate acetate in dimethyl sulfoxide. Staurosporine was supplied by Boehringer Mannheim, (Almere, The Netherlands). Felodipine was kindly supplied by Dr. M. Nordlander (Hässle, Mölndal, Sweden). Bay-K8644 was a generous gift of Dr. S. Kazda (Bayer, FRG). Stock solutions of felodipine and Bay-K8644 (10 $\mathrm{mM}$ ) were dissolved in polyethylene glycol. All further dilutions were performed with double distilled water. Experiments with noradrenaline were performed in the presence of $1 \mu \mathrm{M}$ of the $\beta$-blocking agent propranolol.

\section{Experiments with phorbol myristate acetate}

Concentration response curves for phorbol myristate acetate ( $1 \mathrm{nM}-1$ $\mu \mathrm{M}$ ) were constructed in the presence of $2.5 \mathrm{mM}$ calcium. In addition, concentration response curves for potassium $(10 \mathrm{mM}-125 \mathrm{mM})$ were constructed in the absence or presence of 3,10 or $30 \mathrm{nM}$ phorbol myristate acetate. Furthermore, concentration response curves for noradrenaline were constructed in the absence or presence of $30 \mathrm{nM}$ phorbol myristate acetate. To evaluate whether activation of protein kinase- $C$ could increase the sensitivity of contractile proteins for calcium (Adelstein and Sellers, 1987; Rasmussen et al., 1987), we examined the effects of $3-30 \mathrm{nM}$ phorbol myristate acetate on (i) contractile response to 10 $\mathrm{mM}$ caffeine in calcium-free $\mathrm{KRB}$ and ii) on contractile responses to calcium in vessels that were depleted of intracellular calcium and exposed to $125 \mathrm{mM} \mathrm{K}$ KRB. Furthermore, a cumulative concentration response curve for the dihydropyridine calcium antagonist felodipine was constructed on top of a maximal contractile response to $1 \mu \mathrm{M}$ phorbol myristate acetate. Finally, concentration response curves for phorbol myristate acetate were repeated in the presence of 30 and $100 \mathrm{nM}$ of the dihydropyridine calcium agonist Bay-K8644. 


\section{Effects of staurosporine}

In addition to effects of activation, those of inhibition of protein kinase-C on contractile responses in isolated resistance vessels were also evaluated. Staurosporine, a direct inhibitor of protein kinase-C (Rüegg and Burgess, 1989), was used to suppress the activity of protein kinase-C. Dose response curves to potassium $(5.9-125 \mathrm{mM})$ and noradrenaline $(10 \mathrm{nM}-10 \mu \mathrm{M})$ were constructed in the absence or presence of $0.1,1.0$, or $10 \mathrm{nM}$ staurosporine. Furthermore, the effects of staurosporine on contractile responses to $10 \mathrm{mM}$ caffeine in $\mathrm{Ca}^{2+}$-free $\mathrm{KRB}$ were assessed.

\section{Statistics}

Changes from control induced by phorbol myristate acetate or staurosporine were evaluated using a one-way analysis of variance. In the case of multiple comparisons, one-way ANOVA was followed by a modified t-test according to the method described by Bonferroni (Wallenstein et al., 1980). $P<0.05$ was accepted to denote statistical significance of differences. Contractile responses are expressed as increases in wall tension (force / twice the segment's length). Logistic regression curve fitting (Graphpad Inplot 3.01, San Diego, CA, USA) was performed on concentration response curves to obtain $\mathrm{EC}_{50}$ or $\mathrm{pD}_{2}(=$ $-\log \left[E C_{50}\right]$ ) values.

\section{RESULTS}

\section{Vessel properties}

The optimal lumen diameters of the vessels averaged $208 \pm 5 \mu \mathrm{m} \quad(n=42)$. Contractile responses induced by exposing the sympathectomised vessels to $125 \mathrm{mM} \mathrm{K}-\mathrm{KRB}$ consisted of an initial transient $\left(\mathrm{K}_{\mathrm{t}}\right)$ and a subsequent sustained phase $\left(\mathrm{K}_{\mathrm{s}}\right) . \mathrm{K}_{\mathrm{t}}$ and $\mathrm{K}_{\mathrm{s}}$ averaged $2.64 \pm 0.12 \mathrm{mN} / \mathrm{mm}$, and $2.31 \pm 0.14 \mathrm{mN} / \mathrm{mm}$ $(n=22)$, respectively. As previously observed (Boonen and De Mey, 1990b) both the transient and the sustained response to $125 \mathrm{mM}$ potassium were readily abolished following the removal of extracellular calcium. Noradrenaline induced sustained contractions $\left(\mathrm{pD}_{2} 6.36 \pm 0.07, \mathrm{E}_{\max } 2.68 \pm 0.21 \mathrm{mN} / \mathrm{mm} ; \mathrm{n}=12\right)$ but unlike the reaction to high potassium, a single transient contractile response persisted in the absence of extracellular calcium (Boonen and De Mey, 1990b).

\section{Effects of phorbol myristate acetate}

Phorbol myristate acetate ( $1 \mathrm{nM}-1 \mu \mathrm{M})$ induced slow concentration dependent contractile responses (Fig. 6.1). The maximal contractile response to $1 \mu \mathrm{M}$ phorbol myristate acetate averaged $1.54 \pm 0.37 \mathrm{mN} / \mathrm{mm}(91 \% \pm 14 \%$ of the maximal transient response to $125 \mathrm{mM} \mathrm{K-KRB)}(n=6)$. This response was rapidly abolished by removal of extracellular calcium (not shown) and reduced 


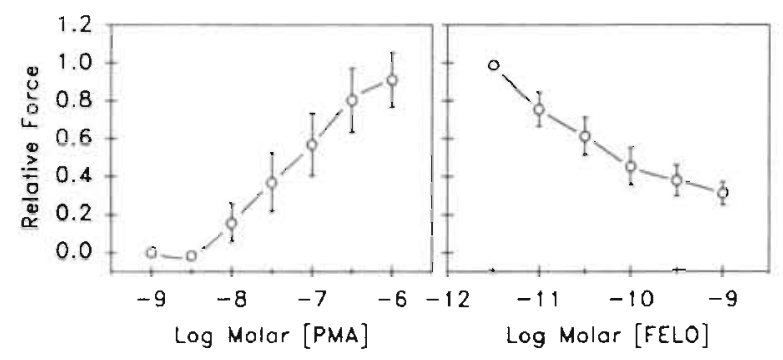

Figure 6.1. Concentration response curve for phorbol myristate acetate (PMA, left panel) in isolated sympathectomised mesenteric resistance arteries in the presence of $2.5 \mathrm{mM}$ calcium. The right panel shows a concentration response curve for felodipine in vessels that had been made to contract with $1 \mu \mathrm{M}$ PMA. Data are expressed as means \pm SEM (left, $\mathrm{n}=6$; right, $\mathrm{n}=8$ ), relative to the maximal contractile response to $125 \mathrm{mM}$ potassium (left) or to the maximal contractile response to $1 \mu \mathrm{M}$ PMA (right).

Figure 6.2. Typical tracing illustrating the effect of $10 \mathrm{nM}$ phorbol myristate acetate on contractile responses to increasing concentrations of potassium in an isolated sympathectomined mesienteric resistance artery (diameter $244 \mu \mathrm{m}$ ). Two curves are displayed, the left one in the absence and the right one in the presence of $10 \mathrm{nM}$ phorbol myristate acetate (hatched bar). The numbers indicate potassium concentrations in $\mathrm{mM}$.

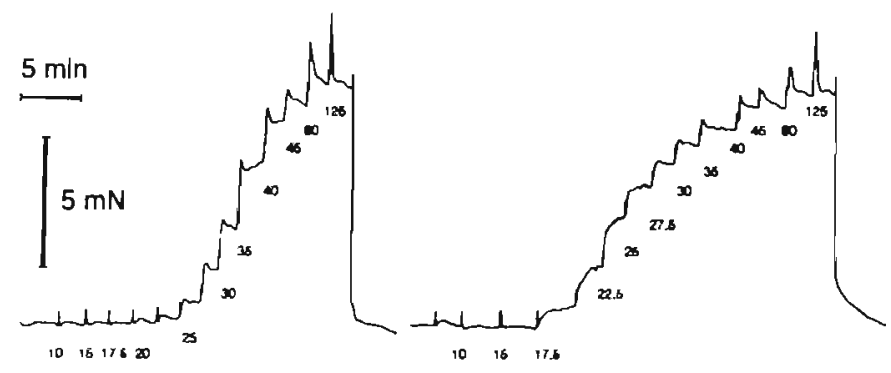

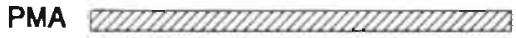

by the dihydropyridine calcium antagonist felodipine $\left(\mathrm{plC}_{50}\right.$ for felodipine averaged $9.40 \pm 0.49(n=10)$ ) (Fig. 6.1). In the continuous presence of $10 \mathrm{nM}$ phorbol myristate acetate, which did not induce a contraction by itself, the maximal sustained, but not the maximal transient response to $125 \mathrm{mM} \mathrm{K}-\mathrm{KRB}$ increased significantly (Table 6.1, Fig. 6.2). More importantly, phorbol myristate acetate increased the sensitivity to the contractile effect of potassium in the presence of $2.5 \mathrm{mM}$ calcium (Table 6.1, Fig. 6.2). This effect was already maximal at $3 \mathrm{nM}$ (Table 6.1). The sensitivity of depolarised vessels to calcium, on 


\section{ROLE OF PROTEIN KINASE-C IN CONTRACTION}

Table 6.1. Effects of phorbol myristate acetate (PMA) on contractile responses to potassium, noradrenaline and calcium in isolated sympathectomised mesenteric resistance sized arteries of the rat.

Concentration response curves for potassium $\left(\mathrm{K}^{*}\right)$ in the presence of $2.5 \mathrm{mM}$ calcium.

\begin{tabular}{lcccc}
\hline & $\begin{array}{c}\mathrm{AWT}_{\text {max }}\left(/ \mathrm{E}_{\max }\right. \\
\text { control) }\end{array}$ & & $\mathrm{EC}_{\text {so }}$ & $\mathbf{n}$ \\
\cline { 2 - 5 } & transient & sustained & & \\
\hline $\mathrm{K}^{+}$control & $1.16 \pm 0.04$ & $0.94 \pm 0.04$ & $30.4 \pm 1.7$ & 14 \\
$\mathrm{~K}^{+}+$PMA 3 n.M & $1.28 \pm 0.17$ & $1.00 \pm 0.03$ & $20.0 \pm 2.3^{\mathrm{a}}$ & 8 \\
$\mathrm{~K}^{+}+$PMA 10 nM & $1.23 \pm 0.06$ & $1.13 \pm 0.07^{\mathrm{a}}$ & $22.5 \pm 1.5^{\mathrm{a}}$ & 6
\end{tabular}

Concentration response curves for noradrenaline (NA) in the presence of $2.5 \mathrm{mM}$ calcium.

\begin{tabular}{lccc}
\hline & $\begin{array}{c}\mathrm{AWT}_{\max }\left(/ \mathrm{E}_{\max }\right. \\
\text { control) }\end{array}$ & $\mathrm{pD}_{2}$ & $\mathbf{n}$ \\
\hline NA control & $0.99 \pm 0.01$ & $6.49 \pm 0.10$ & 12 \\
NA +PMA 30 nM & $1.00 \pm 0.03$ & $6.57 \pm 0.14$ & 6 \\
\hline
\end{tabular}

Top: maximal active wall tension ( $A W T_{\max }$, transient and sustained) and sensitivities to $\mathrm{K}-\mathrm{KRB}\left(E D_{5 n}\right)$ and noradrenaline $\left(\mathrm{pD}_{2}=-\log \left[E C_{50}\right]\right)$ in the absence or presence of PMA. Bottom: Maximal contractions and sensitivities $\left(\mathrm{pD}_{2}\right)$ to calcium in calcium depleted vessels exposed to $125 \mathrm{mM} \mathrm{K-KRB}$ in the absence or presence of PMA. Figures are means \pm SEM $n$ = number of observations. ", denotes significant differences from control (one-way ANOVA + Bonferroni, $\mathrm{P}<0.05 /$ number of comparisons).

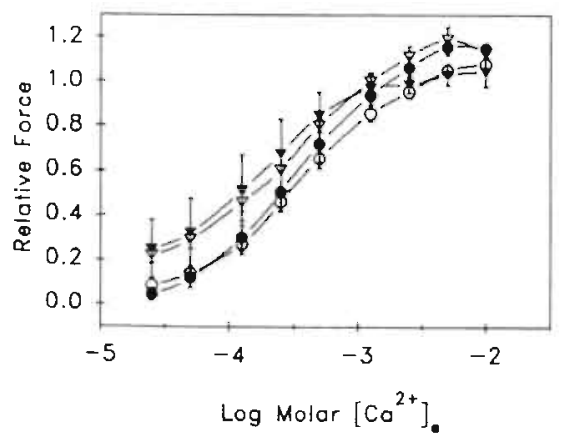

Figure 6.3. Concentration response curves for calcium in mesenteric resistance arteries that had been depleted of calcium and continuously exposed to $125 \mathrm{mM}$ potassium in the absence (open circle) or in the presence of $3 \mathrm{nM}$ (closed circle), $10 \mathrm{nM}$ (open triangle) or $30 \mathrm{nM}$ (closed triangle) phorbol myristate acetate. Data are shown as means \pm SEM as a fraction of the maximal contractile response to $125 \mathrm{mM}$ potassium. 
the other hand, was increased in the presence of 30 , but not of 3 or $10 \mathrm{nM}$ phorbol myristate acetate (Table 6.1, Fig. 6.3). Maximal contractile responses to calcium in depolarised vessels increased only marginally in the presence of 3 and $10 \mathrm{nM}$ phorbol myristate acetate (Table 6.1, Fig. 6.3). $100 \mathrm{nM}$ but not 30 $\mathrm{nM}$ of the calcium channel activator Bay-K8644 induced a contractile response but both $30 \mathrm{nM}$ and $100 \mathrm{nM}$ did not significantly affect contractile responses to phorbol myristate acetate (Fig 6.5.). Contractile responses and sensitivities to noradrenaline were not affected by $30 \mathrm{nM}$ phorbol myristate acetate (Table 6.1). Although threshold concentrations of noradrenaline tended to be affected by phorbol myristate acetate this did not reach significancy. Similarly, contractile responses to $10 \mathrm{mM}$ caffeine in $\mathrm{Ca}^{2+}$-free $\mathrm{KRB}$ were not affected by $30 \mathrm{nM}$ phorbol myristate acetate. They averaged $47.5 \pm 2.0 \%$ and $37.5 \pm 4.0 \%$ of the maximal contractile response to $125 \mathrm{mM}$ potassium in the absence and presence of the phorbol ester, respectively.
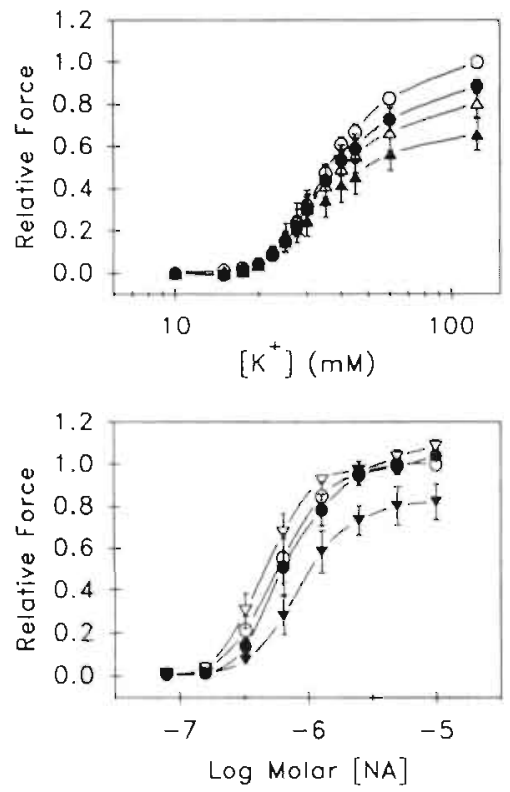

Figure 6.4. Concentration response curves for potassium $\left(\mathrm{K}^{+}\right.$, top) and noradrenaline ( $\mathrm{NA}$, bottom) in the absence (open circle) or presence of 3 (closed circle), $10 \mathrm{nM}$ (open triangle) or $30 \mathrm{nM}$ (closed triangle) staurosporine. Mean values \pm SEM $(n=6)$ are displayed as a fraction of the maximal contractile response to potassium $\left(\mathrm{K}^{+}\right.$, top) or noradrenaline (bottom) in the absence of staurosporine.

\section{Effects of protein kinase-C inhibition}

Staurosporine $(0.1,1.0$ and $10 \mathrm{nM})$ did not affect resting wall tension but significantly reduced maximal contractile responses to potassium in an apparently non-competitive manner (Table 6.2, Fig. 6.4). The protein kinase- $\mathrm{C}$ inhibitor did not affect contractile responses to $10 \mathrm{mM}$ caffeine in $\mathrm{Ca}^{2+}$-free $\mathrm{KRB}$. They averaged $41.8 \pm 4.4 \%$ and $34.1 \pm 2.1 \%(n=10)$ in the absence and presence of $10 \mathrm{nM}$ staurosporine, respectively. Staurosporine $(10 \mathrm{nM})$ tended to reduce maximal contractile responses to noradrenaline, but this did not reach statistical significance (Table 6.2, Fig. 6.4). Staurosporine affected neither the sensitivity to potassium nor that to noradrenaline (Table 6.2). However, concentra- 
tions of staurosporine higher than $100 \mathrm{nM}$ markedly reduced contractile responses to noradrenaline and abolished contractile responses to potassium (data not shown).

Figure 6.5. Concentration response curves for phorbol myristate acetate in isolated sympathectomised mesenteric resistance arteries in the absence (open circles) or in the presence of 100 nM Bay-K8644 (closed circles). Shown are mean values \pm SEM as a fraction of the maximal contractile response to $125 \mathrm{mM}$ potassium. The $\mathrm{pD}_{2}$ for the phorbol ester averaged $7.18 \pm 0.19(n=6)$ and $7.24 \pm 0.22(n=8)$ in the absence or presence of Bay-K8644, respectively.

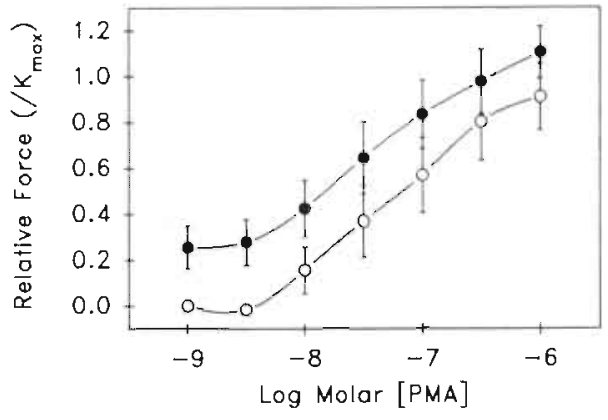

\section{DISCUSSION}

Effects of an activator and of an inhibitor of protein kinase-C on excitation-contraction coupling mechanisms in resistance arteries of the rat were evaluated. For this purpose, isolated sympathectomised mesenteric resistance arteries were stimulated using a depolarising solution or noradrenaline, and the effects of phorbol myristate acetate and staurosporine were evaluated. The result are in agreement with direct observations in vascular smooth muscle cells which suggest that activation of protein kinase- $\mathrm{C}$ can affect the function of voltage operated calcium channels (Gleason and Flaim, 1986; Fish et al., 1988). In this way protein kinase-C could be involved in contractile responses of resistance arterial smooth muscle to depolarisation. Additional effects of protein kinase-C on the calcium sensitivity of the contractile proteins (Adelstein and Sellers, 1987; Drenth et al., 1989; Somlyo and Himpens, 1989; Nelson et al., 1990) could also become involved during powerful activation of the kinase.

\section{Effects of activation of protein kinase-C}

Phorbol myristate acetate induced concentration dependent contractions in mesenteric resistance arteries. This effect has previously been observed in large and small arteries of various origin (Forder et al., 1985; Gleason and Flaim, 1986; Litten et al., 1987; Khalil and Van Breemen, 1988; Mori et al., 1990) and has been attributed to the activation of protein kinase- $C$ by the phorbol ester. It has been suggested that activation of protein kinase-C by vasoactive substances or phorbol esters, increases the sensitivity of myofilaments to calcium (Adelstein and Sellers, 1987; Drenth et al., 1989; Mori et al., 1990; Nishimura et al., 1990). Others suggested that protein kinase- $C$ affects the function of voltage-operated calcium channels by increa- 
Table 6.2. Effects of staurosporine on contractile responses to potassium and noradrenaline in isolated sympathectomised mesenteric resistance arteries of the rat.

Concentration response curves for potassium (K-KRB) in the presence of $2.5 \mathrm{mM}$ calcium

\begin{tabular}{lccc}
\hline & $\begin{array}{c}\text { max AWT }\left(/ E_{\max }\right. \\
\text { control) }\end{array}$ & $\mathrm{EC}_{50}(\mathrm{mM})$ & $\mathbf{n}$ \\
\hline control & $1.00 \pm 0.00$ & $34.7 \pm 5.7$ & 6 \\
+ ST 0.1 nM & $0.89 \pm 0.08$ & $35.9 \pm 6.3$ & 6 \\
+ ST $1.0 \mathrm{nM}$ & $0.81 \pm 0.16$ & $35.6 \pm 7.2$ & 6 \\
+ ST $10 \mathrm{nM}$ & $0.66 \pm 0.19^{a}$ & $36.1 \pm 6.7$ & 6 \\
\hline Concentration response curves for noradrenaline in the presence of $2.5 \mathrm{mM}$ calcium \\
\hline
\end{tabular}

Maximal contractile responses (max AWT) and sensitivities to potassium (top) and noradrenaline (bottom) in the absence (control) or presence of staurosporine (ST). Control $E_{\max }$ averaged $2.21 \pm 0.55 \mathrm{mN} / \mathrm{mm}$ for $K_{1}$ and $2.61 \pm 0.82 \mathrm{mN} / \mathrm{mm}$ for noradrenaline. AWT values in the presence of staurosporine are displayed as fraction of $E_{\max }$ of their respective controls. " ; denotes significant differences from control (one-way ANOVA + Bonferroni, $P<0.05 /$ number of comparisons). $n=$ number of observations.

sing the probability of these channels to open (Gleason and Flaim, 1986; Jiang and Morgan, 1987; Rasmussen et al., 1987; Khalil and Van Breemen, 1988).

In this, as in other studies (Forder et al., 1985; Litten et al., 1987; Fish et al., 1988) contractions to phorbol myristate acetate depended on the presence of extracellular calcium. This suggests the involvement of calcium influx. In addition, contractions in response to $1 \mu \mathrm{M}$ phorbol myristate acetate were reduced by the dihydropyridine calcium antagonist felodipine with a potency that lies between those for inhibition of responses to high potassium and noradrenaline (Nyborg and Mulvany, 1984). This latter observation suggests an effect on L-type voltage-operated calcium channels. Noradrenaline as well as phorbol myristate acetate could, through the activation of protein kinase- $C$, increase the probability of voltage operated calcium channels to be in an open state (Fish et al., 1988; Nelson et al., 1988; Boonen and De Mey, 1990b). However, part of the contractile response to phorbol myristate acetate was resistant to felodipine indicating that activation of protein kinase- $\mathrm{C}$ could also have additional effects on the contractile 
machinery (Jiang and Morgan, 1987; Drenth et al., 1989; Mori et al., 1990; Nishimura et al., 1990).

Contractile responses to noradrenaline were not affected even by $30 \mathrm{nM}$ phorbol myristate acetate (Table 6.1). As mentioned before, both noradrenaline and phorbol myristate acetate are potent activators of protein kinase-C (Berridge, 1986; Nishizuka, 1986; Van Breemen and Saida, 1989). This could explain the lack of effect of phorbol myristate acetate on responses to noradrenaline, because the activation of protein kinase- $\mathrm{C}$ by phorbol myristate acetate would be overwhelmed by the massive activation of protein kinase- $C$ by noradrenaline. Similar observations were reported by Drenth et al., (1989) and Nishimura et al., (1990).

Particularly interesting, however, is the observation that in the presence of low concentrations of phorbol myristate acetate, the vessels were hypersensitive to the contractile effect of potassium, but not to that of calcium in depolarised vessels (Fig. 6.2, Table 6.1). This effect, which was maximal at concentrations as low as $3 \mathrm{nM}$ phorbol myristate acetate, is in accordance with the hypothesis that voltage-sensitive calcium channels are affected by phorbol myristate acetate. However, the calcium agonist Bay-K8644, which activates voltage operated calcium channels (Nelson et al., 1990), had no significant effect on contractile responses to phorbol myristate acetate (Fig. 6.5). Contrary to this observation Litten et al., (1987) found that the presence of the calcium channel agonist Bay-K8644 allows protein kinase-C to potentiate the mobilisation of calcium through L-type calcium channels in rat aorta. First, the discrepancy between effects of activation of protein kinase- $C$ on contractile responses to potassium or Bay-K8644 could indicate that some depolarisation is required for protein kinase-C to affect calcium channels. In this respect our indirect observations are compatible with the findings of Fish et al., (1988) that phorbol ester increases the conductance of L-type calcium channels in a vascular smooth muscle cell line at a patch pipette holding potential of $-30 \mathrm{mV}$. Secondly, the differences between effects of Bay-K8644 on contractile responses induced by phorbol esters in small arteries (this study) and aorta (Litten et al., 1987) could be explained by the fact that dihydropyridines act with different selectivity and specificity in large and small arteries (Cauvin et al., 1985).

Another possibility by which activation of protein kinase- $C$ could affect contractile responses to a depolarising stimulus is through inhibitory effects of phorbol myristate acetate on the endothelial release of endothelium derived relaxing factor (EDRF) (Morrison and Pollock, 1990) or by stimulation of the release of endothelium derived contracting factors. However, endothelium removal, arginine analogues that inhibit EDRF release, methylene blue or indomethacin do not alter the basal tension or sensitivity to potassium in isolated mesenteric resistance arteries (De Mey and Boonen, unpublished observations).

To evaluate the possibility that protein kinase-C increases the calcium sensitivity of vascular smooth muscle myofilaments (Jiang and Morgan, 1987; Drenth et al., 1989; Mori et al., 1990; Nishimura et al., 1990), calcium concentration response curves were constructed in maximally depolarised arteries. The sensitivity to calcium was increased in the presence of $30 \mathrm{nM}$ phorbol myristate acetate, but 
not 3 or $10 \mathrm{nM}$. Contractile responses to caffeine in the absence of extracellular calcium which are due predominately to the release of intracellular calcium (Van Breemen and Saida, 1989), were not affected by $30 \mathrm{nM}$ phorbol myristate acetate. Taken together, our results suggest that protein kinase- $C$ increases calcium sensitivity but that this occurs only at concentrations of phorbol myristate acetate which are ten times higher than those needed to affect the sensitivity to a depolarising stimulus. Therefore, this latter effect seems to be primarily due to an effect of phorbol myristate acetate on the function of calcium channels, with a calcium sensitizing effect arising only at higher concentrations of phorbol myristate acetate. Whether this represents true differences between membrane bound and cytoplasmic kinases or their substrates (Fabbro et al., 1988; Ho et al., 1988), or whether extracellularly applied phorbol myristate acetate has easier access to the cell membrane than to the cytoplasm, is unclear. Nevertheless, there are indications that protein kinase- $\mathrm{C}$ is translocated to the plasma membrane when it is activated (Fabbro et al., 1988; Ho et al., 1988), which means that the differences in the effects of phorbol myristate acetate on calcium influx pathways and calcium sensitisation of contractile proteins could be due to the location of the kinase.

\section{Effects of inhibition of protein kinase-C}

Both phospholipase- $C$ and protein kinase- $C$ can be activated by calcium (Berridgc, 1986; Nishizuka, 1986; Ho et al., 1988), raising the possibility that calcium influx following depolarisation could stimulate these enzymes. In view of the above, this could further promote calcium entry and sensitise the contractile machinery for the cation. To test this hypothesis we recorded effects of staurosporine, an inhibitor of protein kinase-C on contractile responses to potassium and noradrenaline.

Staurosporine significantly reduced contractile responses to potassium. However, contractile responses following $\alpha_{1}$-adrenergic stimulation were not significantly affected by concentrations of staurosporine that affected those to potassium. A possible explanation is that contractile responses to $\alpha_{1}$-adrenergic stimulation do not solely rely on activation of protein kinase- $C$ but also involve different processes such as activation of inositol triphosphate (Berridge, 1986; Somlyo and Himpens, 1989; Van Breemen and Saida, 1989; Raymond et al., 1990). Also contractile responses to caffeine in calcium free medium were not affected by staurosporine. However, high concentrations ( $\geq 100 \mathrm{nM}$ ) of staurosporine completely blocked contractile responses to potassium as well as those following $\alpha_{1}$-adrenergic stimulation, suggesting that high concentrations of staurosporine could have effects on enzymes, other than protein kinase-C, that are involved in contractile responses (Rüegg and Burgess, 1989). Furthermore, Buchholz et al., (1991) have reported that straurosporine in low concentrations (below $60 \mathrm{nM}$ ) selectively inhibits protein kinase-C whereas at higher concentrations (above $70 \mathrm{nM}$ ) inhibits myosin light chain kinase as well. Taking these dual properties of staurosporine into account, the effects of staurosporine at low concentrations are in agreement with the hypothesis that calcium influx following depolarisa- 
tion can activate protein kinase-C, which in turn plays a role in further sustaining calcium influx. This, however, needs to be firmly established in future experiments.

To recapitulate, we evaluated effects of an activator and inhibitor of protein kinase- $\mathrm{C}$ on contractile responses to depolarisation, agonists and calcium in isolated mesenteric resistance sized arteries of the rat. We conclude that, provided phorbol myristate actetates activates protein kinase-C (Nishizuka, 1986), this kinase could be involved in contractile responses to depolarising stimuli. The results confirm direct observations in vascular smooth muscle cells which suggest that protein kinase-C could play a role in the activation of voltage operated calcium channels (Gleason and Flaim, 1986; Jiang and Morgan, 1987; Fish et al., 1988). Effects of protein kinase-C on contractile proteins may be involved during strong activation of the kinase. These data are compatible with the hypothesis of vasoconstrictor agonists having an effect on voltage-operated calcium channels through activation of protein kinase-C (Boonen and De Mey, 1990b). Direct observations are required to support this hypothesis. 



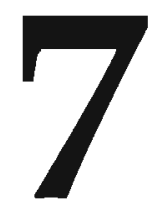

\section{Length-Dependency of Excitation- Contraction Coupling in Isolated Mesenteric Resistance Arteries}

Harrie C.M. Boonen and Jo G.R. De Mey

Department of pharmacology, University of Limburg, Maastricht, the Netherlands. 


\title{
SUMMARY
}

\begin{abstract}
Busides electro- and pharmacomechanical stimulation, mechanical forces may affect excitation-contraction coupling mechanisms in resistance arteries. In this way alterations in pressure or flow may be transduced into mechanical responses of the vascular smooth muscle cell. We evaluated effects of distension on responses to vasoconstrictors and vasodilators in isolated mesenteric small arteries of normotensive and genetically hypertensive rats. Vessel segments were isolated, chemically sympathectomised and mounted for recording of isometric force development. At diameters 30\% lower $(0.7$ $\left.D_{0}\right)$ or $30 \%$ higher $\left(1.3 D_{0}\right.$ ) than the diameter $\left(D_{0}\right)$ at which vessels responded maximally to $125 \mathrm{mM}$ potassium (KRB), concentration response curves were constructed for potassium, noradrenaline (NA) and extracellular calcium. Vessels of both WKY and SHR showed a non-selective hypersensitivity to these vasoconstrictors at $1.3 D_{0}$. Hypersensitivity to potassium at $1.3 \mathrm{D}_{0}$ persisted in the absence of the endothelium. Inhibition of maximal contractile responses to K-KRB or NA by felodipine was comparable at $0.7 D_{0}$ and $1.3 \mathrm{D}_{0}$. The calcium channel activator Bay K8644 caused contraction in part of the vessels at $1.3 \mathrm{D}_{0}$, but in none of the vessels at $0.7 \mathrm{D}_{0}$. Furthermore, BRL-38227, a potassium channel activator, reduced responses to NA less potently at $1.3 \mathrm{D}_{0}$ than at $0.7 \mathrm{D}_{0}$. Also relaxing responses to potassium in sodium loaded preparations made to contract with NA tended to be less marked at $1.3 \mathrm{D}_{0}$ than at $0.7 \mathrm{D}_{0}$. These findings suggest that in small arteries, distension i) decreases membrane potential of the arterial smooth muscle cells and ii), increases the sensitivity for calcium during maximal depolarisation.
\end{abstract}

\section{INTRODUCTION}

Classically, electromechanical and pharmacomechanical coupling are believed to regulate vascular smooth muscle cell reactivity on a cellular level (Somlyo and Somlyo, 1968; Van Breemen et al., 1986). In the former a depolarising stimulus is converted into a mechanical response through opening of voltage operated calcium channels in the cell membrane and subsequent influx of calcium (Van Breemen et al., 1986). During pharmacomechanical coupling, activation of membrane surface receptors leads to activation of calcium channels and release of calcium from intracellular stores through the action of G-proteins and second messenger systems (Somlyo and Somlyo, 1968; Van Breemen et al., 1986). In resistance arterial smooth muscle these pathways are not strictly separated but may interdigitate (Boonen and De Mey, 1990; Mulvany and Aalkjaer, 1990; Nelson et al., 1990). Besides these excitation-contraction coupling mechanisms contractile responses of vascular smooth muscle can also be elicited by dynamic or static stretch and by increasing transmural pressure (Johnson, 1980; Laher and Bevan, 1989; Harris and Warshaw, 1991). The mechanisms that are responsible for the transduction of a mechanical stimulus into a contractile response in vascular smooth muscle are largely unknown.

Some reports suggest the existence of stretch-operated calcium channels in the cellmembrane which have characteristics that differ from those of voltage-operated- and receptor-operated calcium channels (Hwa and Bevan, 1986a; 
Kirber et al., 1988). During dynamic stretch of vascular smooth muscle these channels would be activated to promote the influx of calcium. However, the stretchinduced contractile response is but poorly maintained as soon as stretching is halted (Hwa and Bevan, 1986b). Others found effects of stretch on components of the contractile apparatus (Moreland et al., 1988; Barany et al., 1990), on the activity of second messengers (Watson, 1991) and on the excitability of the cellmembrane (Johansson, 1987; Kirber et al., 1988). Furthermore, the endothelium may play a role in the stretch-induced reactivity of vascular smooth muscle (Griffith and Edwards, 1990). These effects of dynamic stretch may play a role in the myogenic autoregulatory response in resistance-sized arteries (Johnson, 1980). Previously it has been shown that static distension of arterial smooth muscle affects the sensitivity to vasoconstrictors (Price et al., 1981; Nilsson and Sjöblom, 1985). This phenomenon may also be involved in functional autoregulatory responses in arterial smooth muscle (Johnson, 1980; Johansson, 1989; Meininger et al., 1991). Small arteries that significantly contribute to peripheral resistance show rhythmic vasomotion, the frequency of which increases with increasing pressure (Burrows and Johnson, 1981). In spontaneously hypertensive but not in normotensive Wistar Kyoto rats rhythmicity of contractile responses was observed in isolated small arteries (Boonen and De Mey, 1990). Whether established differences in transmural pressure between adult spontaneously hypertensive and normotensive Wistar Kyoto rats in vivo, could result in changes in mechanotransducing pathways or vice versa is unknown.

In the present study we therefore evaluated the effects of distension of small artery smooth muscle of spontaneously hypertensive and normotensive Wistar Kyoto rats on the reactivity to vasoconstrictors and extracellular calcium. We assessed whether the endothelium, calcium channels or membrane potential may be involved in the distension-dependent hypersensitivity to vasoconstrictors using pharmacological tools.

\section{MATERIAL AND METHODS}

\section{Preparation of vessels}

Adult male Wistar Kyoto (WKY) and age and sex matched spontaneously hypertensive rats (SHR) were used (locally inbred strains, University of Limburg, Maastricht, the Netherlands). Rats were sacrificed by cervical dislocation and exsanguination. The mesentery was isolated and immersed in KrebsRinger bicarbonate buffered salt solution. A fourth order side branch of the superior mesenteric artery was cleaned of fat and connective tissue. Sympathetic nerve endings were chemically destroyed by $10 \mathrm{~min}$. incubation at $37^{\circ} \mathrm{C}$ in bicarbonate-free Krebs solution that contained $300 \mu \mathrm{g} / \mathrm{ml}$ 6-hydroxydopamine (Aprigliano and Hermsmeyer, 1977). During isolation, mounting and experimentation, the vessels were immersed in Krebs solution which had the following composition (in $\mathrm{mM}$ ): $\mathrm{NaCl} 118.5 ; \mathrm{KCl} 4.7 ; \mathrm{MgSO}_{4} 1.2 ; \mathrm{KH}_{2} \mathrm{PO}_{4} 1.2 ; \mathrm{NaHCO}_{3} 25.0$; 
$\mathrm{CaCl}_{2}$ 2.5; glucose 11.1. High potassium solution (K-KRB), was prepared by replacing all the $\mathrm{NaCl}$ in normal $\mathrm{KRB}$ with an equimolar amount of $\mathrm{KCl}$. Intermediate potassium concentrations were obtained by mixing appropriate amounts of $\mathrm{KRB}$ and $\mathrm{K}-\mathrm{KRB} . \mathrm{Ca}^{2+}$-free $\mathrm{KRB}$ and $\mathrm{Ca}^{2+}$-free $\mathrm{K}-\mathrm{KRB}$ were obtained by omitting $\mathrm{CaCl}_{2}$ from $\mathrm{KRB}$ and $\mathrm{K}-\mathrm{KRB}$, respectively and including $0.3 \mathrm{mM}$ EGTA.

Vessel segments (approximately $2 \mathrm{~mm}$ in length) were threaded on two stainless steel wires $(\varnothing 40 \mu \mathrm{m})$ and mounted as ring segments between a displacement device and a force transducer (Kistler-Morse DSC6, Seattle, USA) in an isometric myograph (Mulvany and Halpern, 1977). Vessels were initially subjected to a length-tension protocol (see Chapter 2). This consisted of stepwise stretching and intermittently stimulating the vessel with $125 \mathrm{mM} \mathrm{K-KRB}$. In this way an optimal lumen diameter $\left(D_{0}\right)$ could be obtained at which the vessel responded maximally to $125 \mathrm{mM} \mathrm{K-KRB}$. For subsequent experimentation, vessels were set at a diameter that was either below $\left(0.7\right.$ times $\left.D_{0}\right)$ or above $\left(1.3\right.$ times $\left.D_{0}\right)$ the optimal diameter. Preliminary observations showed that at these two points on the circumferential length-tension curve the maximal force development was similar. Two vessels of one animal of which one was stretched to $0.7 \mathrm{D}_{0}$ and one to $1.3 \mathrm{D}_{0}$ were studied in parallel in the same myograph.

\section{Experimental protocols}

Cumulative concentration response curves were constructed for potassium $(5.9-125 \mathrm{mM})$, and noradrenaline $(10 \mathrm{nM}-10 \mu \mathrm{M})$ in the continuous presence of $2.5 \mathrm{mM}$ calcium in vessels of WKY and SHR that were either set at $0.7 D_{1}$ or $1.3 D_{0}$. Concentration response curves for noradrenaline were constructed in the presence of $1 \mu \mathrm{M}$ of the $\beta$-blocking agent propranolol. In some experiments, concentration response curves for potassium were repeated in vessels of WKY from which the endothelium had been removed mechanically (De Mey et al., 1991).

To assess effects of distension on the sensitivity for extracellular calcium, concentration response curves were constructed for the divalent cation Boonen and De Mey, 1990) in the continuous presence of $125 \mathrm{mM} \mathrm{K-KRB}$. Furthermore, the effect of distension on the function of voltage operated calcium channels was evaluated indirectly by the use of the dihydropyridine calcium channel antagonist felodipine and -agonist Bay K8644. To obtain concentration response curves for felodipine, contractile responses to $125 \mathrm{mM} \mathrm{K}-\mathrm{KRB}$ and $10 \mu \mathrm{M}$ noradrenaline were repeated in the absence and presence of increasing concentrations of felodipine (1 pM - $1 \mathrm{nM})$. Those for Bay K8644 $(0.1 \mathrm{nM}-1 \mu \mathrm{M})$ were performed in resting preparations.

The role of membrane potential in the distension-dependent sensitivity was evaluated by indirect approaches. Relaxing responses to hyperpolarising interventions were recorded. We therefore administered the potassium channel opener BRL-38227 (l-isomer of cromakalim) on top of a maximal contractile response to $10 \mu \mathrm{M}$ noradrenaline. Secondly, at $0.7 \mathrm{D}_{0}$ and $1.3 \mathrm{D}_{0}$, concentration response curves for potassium and noradrenaline were repeated in the presen- 
ce of $1 \mu \mathrm{M}$ and $3 \mu \mathrm{M}$ BRL-38227. Finally, potassium induced relaxations were performed by incubating vessels for 10 minutes in KRB solution from which $\mathrm{KCl}$ was omitted $\left(\left[\mathrm{K}^{+}\right]_{\mathrm{e}} 1.2 \mathrm{mM}\right)$. Then a contractile response to $10 \mu \mathrm{M}$ noradrenaline was induced and after reaching a steady state contractile response, $\left[\mathrm{K}^{+}\right]_{\mathrm{e}}$ was restored to the control level $(5.9 \mathrm{mM})$. This resulted in a transient relaxation that has been attributed to activation of the electrogenic sodium pump (De Mey and Vanhoutte, 1982).

\section{Drugs and solutions}

6-Hydroxydopamine, noradrenaline (l-arterenol bitartrate) and propranolol $\mathrm{HCl}$ were purchased from Sigma Chemicals (Saint Louis, MO, USA). BRL-38227 was a generous gift from Dr. R. Buckingham (SmithKline Beecham, Harlow, Essex, UK). Felodipine was kindly supplied by Dr. M. Nordlander (Hässle, Mölndal, Sweden). Bay K8644 was a gift of Dr. Kazda (Bayer, FRG). The dihydropyridines were dissolved as stock solutions $(10 \mu \mathrm{M})$ in polyethyleneglycol and stored at $4^{\circ} \mathrm{C}$. From these, dilutions were prepared daily. All other solutions were freshly prepared in bidistilled water on the day of experimentation.

\section{Data analysis}

Sensitivities for the vasoconstrictors potassium, noradrenaline and extracellular calcium and the vasodilators felodipine and BRL-38227 were determined from the concentration response curves using a least square sigmoidal curve fit (Graphpad Software, San Diego, CA, USA). Differences between observations at the two distensions or between WKY and SHR with respect to maximal effects and sensitivities for the vasoconstrictors were evaluated with Student's t-test. Effects of BRL-38227 were analysed by two-way analysis of variance (ANOVA) followed by t-test according to Bonferroni (Wallenstein et al., 1980). Statistical analysis was performed using Crunch Interactive Statistical Package (CRUNCH, Software Corp., San Francisco, CA, USA).

\section{RESULTS}

Changing the diameter of isolated sympathectomised mesenteric small arteries altered resting wall tension, active wall tension and sensitivity for vasoconstrictor stimuli. In resistance arteries exposed to KRB, stepwise increases in diameter resulted in progressively larger increases in wall tension. The levels reached after stress relaxation were not affected by removal of extracellular calcium or by the presence of up to $1 \mu \mathrm{M}$ of the dihydropyridine calcium antagonist felodipine suggesting fully relaxed conditions. Resting wall tension (RWT) in vessels of WKY averaged $0.22 \pm 0.06 \mathrm{mN} / \mathrm{mm}$ and $5.27 \pm 1.29$ $\mathrm{mN} / \mathrm{mm}$ at $0.7 \mathrm{D}_{0}$ and $1.3 \mathrm{D}_{0}$, respectively $(\mathrm{n}=16)$. These were not different in 

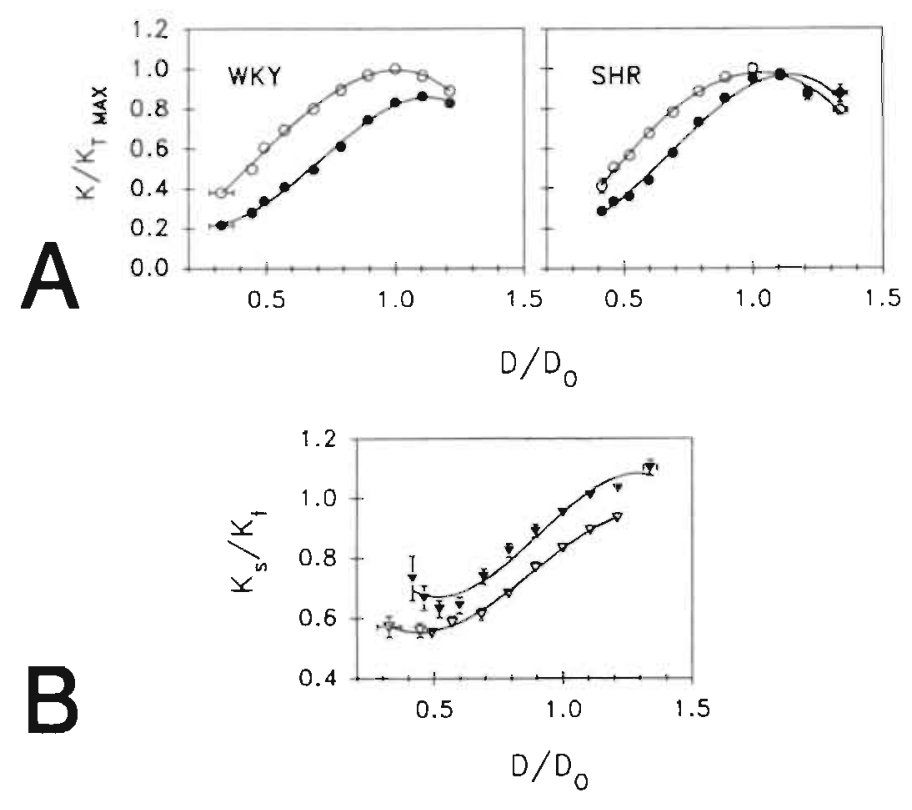

Figure $7.1 \mathrm{~A})$ Diameter-tension relationship in small mesenteric arteries (upper panels) from Wistar Kyoto (WKY) or spontaneously hypertensive rats (SHR). Displayed are transient (open circles) and sustained (filled circles) contractile responses to $125 \mathrm{mM} \mathrm{K}$ $\mathrm{KRB}$ as a fraction of the maximal transient response to $125 \mathrm{mM} \mathrm{K-KRB}$. Diameter is expressed as a fraction of the optimal lumen diameter at which the vessel contracted maximally in response to $125 \mathrm{mM} \mathrm{K}-\mathrm{KRB}$.

B) The ratio between sustained and transient response to $125 \mathrm{mM} \mathrm{K-KRB}\left(\mathrm{K}_{\mathrm{s}} / \mathrm{K}_{\mathrm{t}}\right)$ displayed in relation to the diameter $\left(D\right.$; as a fraction of $D_{0}$ ) of vessels of WKY (open triangles) and SHR (filled triangles). Values are means \pm SEM (WKY; $n=32, S H R ; n=31$ ).

vessels of SHR (RWT averaged: $0.36 \pm 0.07 \mathrm{mN} / \mathrm{mm}$ at $0.7 \mathrm{D}_{0}$ and $5.34 \pm 0.71$ $\mathrm{mN} / \mathrm{mm}$ at $\left.1.3 \mathrm{D}_{0}(\mathrm{n}=16)\right)$. Stimulation of small arteries at different diameters with $125 \mathrm{mM} \mathrm{K-KRB}$ resulted in a biphasic contractile response consisting of an initial transient $\left(\mathrm{K}_{\mathrm{t}}\right)$ and a subsequent sustained response $\left(\mathrm{K}_{\mathrm{s}}\right)$ (Fig's. 7.1 and 2.3). Changes in amplitude of $K_{1}$ with increasing diameters were roughly symmetrical around $\mathrm{D}_{0}$. At $0.7 \mathrm{D}_{0}$ and $1.3 \mathrm{D}_{0}, \mathrm{~K}_{4}$ averaged $74.0 \pm 6.3 \%(\mathrm{n}=8)$ and $88.4 \pm 4.2 \%(n=8)$ of that at $D_{0}$ in vessels of WKY. These conditions of comparable active tension at different diameter and different resting tension were selected for further experimentation. The sustained contractile response $\left(\mathrm{K}_{s}\right)$ to $125 \mathrm{mM} \mathrm{K}-\mathrm{KRB}$ not only changed in amplitude but also in relationship to $\mathrm{K}_{t}$ (Fig.7.1 and Fig. 2.3). $\mathrm{K}_{\mathrm{s}} / \mathrm{K}_{\mathrm{t}}$ averaged $0.62 \pm 0.05$ and $0.93 \pm 0.03$ in vessels of WKY and $0.80 \pm 0.07$ and $1.05 \pm 0.03$ in vessels of SHR at $0.7 D_{0}$ and $1.3 D_{0}$ respectively. $\mathrm{K}_{s} / \mathrm{K}_{\mathrm{t}}$ was significantly lower at $0.7 \mathrm{D}_{0}$ than at $1.3 \mathrm{D}_{0}$ in both WKY 
and SHR. Along the entire diameter-active tension curve $K_{s} / K_{t}$ was significantly higher in SHR than in WKY (Fig. 7.1).
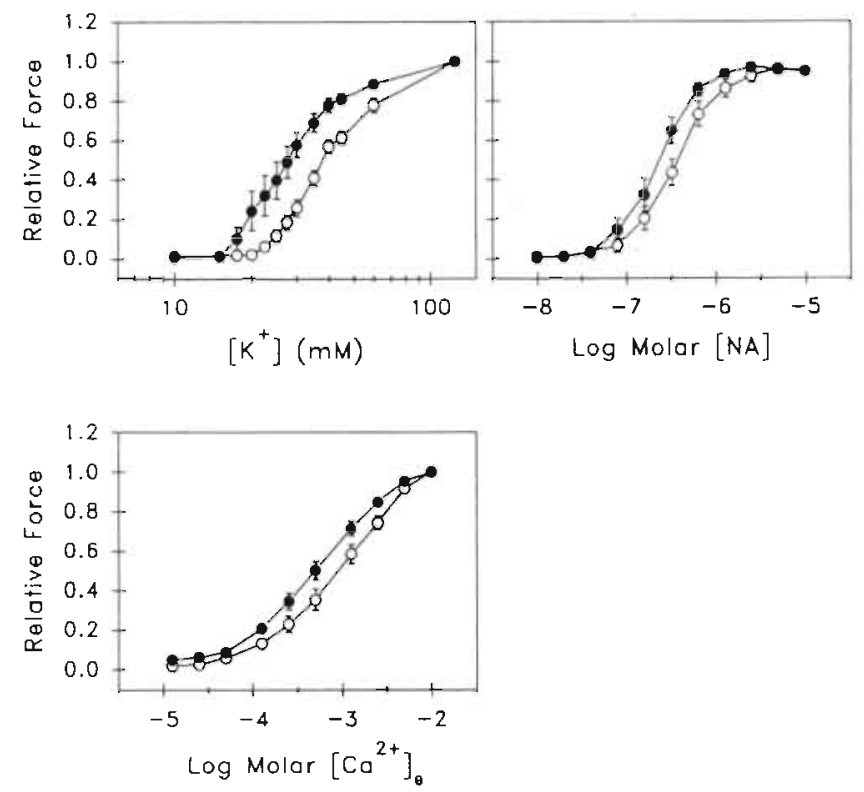

Figure 7.2 Concentration response curves for potassium $\left(\left[\mathrm{K}^{*}\right]\right)$, noradrenaline (NA) and extracellular calcium $\left(\left[\mathrm{Ca}^{2 \cdot}\right]_{\vec{r}}\right)$ in isolated sympathectomised mesenteric small arteries of WKY rats that were distended to either 0.7 times (open circles) or 1.3 times the optimal lumen diameter $\left(D_{0}\right)$. Concentration response curves to $N A$ were constructed in the presence of $1 \mu \mathrm{M}$ propranolol, those to [C $\left.\mathrm{Ca}^{2 *}\right]_{1}$, in the presence of $125 \mathrm{mM} \mathrm{K-KRB}$. Values are mean $\pm S E M$ as a fraction of the respective maximal response to the used stimulus.

\section{Effects of distension on sensitivity of vasoconstrictors}

Vessels of both WKY and SHR were hypersensitive to the contractile effects of potassium, noradrenaline and extracellular calcium at $1.3 \mathrm{D}_{0}$ compared to $0.7 D_{0}$ (Fig. 7.2 and Table 7.1). Previously reported values at $D_{0}$ were intermediate between the present observations at $0.7 \mathrm{D}_{0}$ and $1.3 \mathrm{D}_{0}$ (see Chapters 2-6). Mechanical removal of the endothelium did not affect maximal contractile responses to $125 \mathrm{mM} \mathrm{K-KRB} \mathrm{(Table} \mathrm{7.1)} \mathrm{but} \mathrm{abolished} \mathrm{relaxing} \mathrm{responses} \mathrm{to} 1$ $\mu \mathrm{M}$ acetylcholine in vessels that were precontracted with $1 \mu \mathrm{M}$ noradrenaline. Removal of the endothelium, however, had no significant effect on the change in sensitivity for potassium following distension of the vessels (Table 7.1).

Changes in sensitivity for noradrenaline as well as potassium and extracellular calcium in maximally depolarised preparations can be due to effects of distension on the function of voltage operated calcium channels. This pos- 


\section{CHAPTER 7}

sibility was assessed by an indirect approach. The efficacy of the dihydropyridine calcium antagonist felodipine to inhibit contractile responses to $125 \mathrm{mM}$ potassium and $10 \mu \mathrm{M}$ noradrenaline were not affected by distension (Fig 7.3). However, the dihydropyridine calcium agonist Bay K8644 induced contractile responses in vessels that were set at $1.3 \mathrm{D}_{0}$ but in none of the vessels at $0.7 \mathrm{D}_{0}$ (Fig 7.3).

Table 7.1. Fffects of distension on maximal contractile responses and sensitivities for potassium, noradrenaline and extracellular calcium in isolated mesenteric resistance arteries of $W K Y$ and $S H R$.

\begin{tabular}{|c|c|c|c|c|}
\hline & \multicolumn{2}{|c|}{ WKY } & \multicolumn{2}{|c|}{ SHR. } \\
\hline & $0.7 \mathrm{D}_{0}$ & $1.3 \mathrm{D}_{0}$ & $0.7 \mathrm{D}_{0}$ & $1.3 \mathrm{D}_{0}$ \\
\hline \multicolumn{5}{|l|}{ Potassium } \\
\hline$A W T_{1 \mathrm{man}}(\mathrm{mN} / \mathrm{mm})$ & $1.54 \pm 0.23(9)$ & $1.62 \pm 0.26(9)$ & $2.50 \pm 0.30^{b}(8)$ & $2.61 \pm 0.2^{b}(8)$ \\
\hline$A W T_{s \max }(\mathrm{mN} / \mathrm{mm})$ & $1.00 \pm 0.20(9)$ & $1.47 \pm 0.21(9)$ & $2.10 \pm 0.33^{b}(8)$ & $2.71 \pm 0.26^{b}(8)$ \\
\hline $\mathrm{EC}_{50} \quad(\mathrm{mM})$ & $34.0 \pm 1.1(9)$ & $25.3 \pm 1.8^{\mathrm{a}}(9)$ & $34.6 \pm 1.7(8)$ & $24.1 \pm 1.4^{\mathrm{a}}(8)$ \\
\hline \multicolumn{5}{|c|}{ Potassium (in vessels denuded of the endothelium) } \\
\hline$A W T_{1 \max }(\mathrm{mN} / \mathrm{mm})$ & $1.43 \pm 0.16(7)$ & $1.68 \pm 0.35(n)$ & n.d. & n.d. \\
\hline $\mathrm{AWT}_{\mathrm{s} \max }(\mathrm{mN} / \mathrm{mm})$ & $1.15 \pm 0.14(7)$ & $1.41 \pm 0.33(7)$ & n.d. & n.d. \\
\hline $\mathrm{EC}_{\mathrm{*},} \quad(\mathrm{mM})$ & $39.2 \pm 1.9(7)$ & $29.1 \pm 2.8^{\circ}(7)$ & n.d. & n.d. \\
\hline \multicolumn{5}{|l|}{ Noradrenaline } \\
\hline$A W T_{\operatorname{mixx}}(\mathrm{mN} / \mathrm{mm})$ & $2.99 \pm 0.18(17)$ & $2.24 \pm 0.22^{b}(17)$ & $3.63 \pm 0.45^{b}(8)$ & $3.59 \pm 0.33^{b}(8)$ \\
\hline $\mathrm{pD}_{2}$ & $6.36 \pm 0.12(17)$ & $6.84 \pm 0.14^{x}(17)$ & $6.51 \pm 0.04(8)$ & $6.85 \pm 0.10^{2}(8)$ \\
\hline \multicolumn{5}{|l|}{ Calcium + K125 } \\
\hline $\mathrm{pCa}_{2}$ & $2.90 \pm 0.13(9)$ & $3.23 \pm 0.10^{\mathrm{x}}(9)$ & $2.90 \pm 0.19(8)$ & $\begin{array}{c}3.33 \pm 0.20^{3} \\
\text { (8) }\end{array}$ \\
\hline
\end{tabular}

Active wall tension $\left(A W T_{t_{\max }}\right.$ and $A W T_{m_{\max }}$ : transient and sustained phases in the biphasic response to K-KRB) displayed as maximal force development per twice vessel axial length. Sensitivities for to the vasoconstrictors given as $\mathrm{EC}_{50}(\mathrm{~K}-\mathrm{KRB})$ or as the negative logarithm of the $\mathrm{EC}_{\mathrm{s}}\left(\mathrm{pD}_{2}\right.$ and $\mathrm{pCa}_{2}$, respectively). Concentration response curves to $\left[\mathrm{Ca}^{2 *}\right]_{\mathrm{e}}$ were obtained in maximally depolarised preparations (125 $\mathrm{mM} \mathrm{K-KRB).} \mathrm{Those} \mathrm{to}$ noradrenaline were constructed in the presence of $1 \mu \mathrm{M}$ propranolol. Values are mean \pm SEM. Number of observations: $(n)$. "and " represent significant differences between vessels at $0.7\left(0.7 D_{0}\right)$ and $1.3\left(1.3 D_{0}\right)$ times the optimal lumen diameter $\left(D_{0}\right)$ or between WKY and SHR, respectively. 

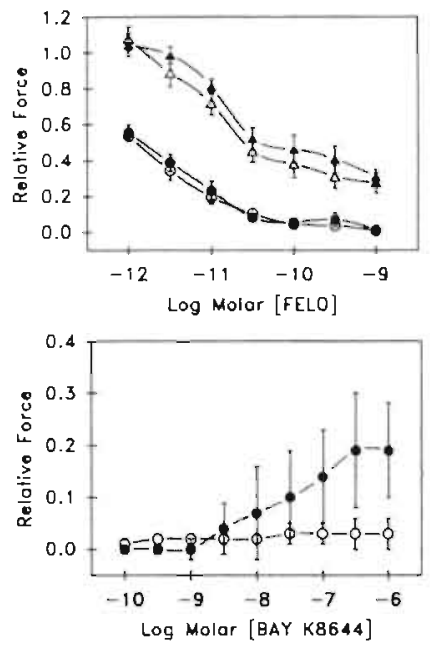

Figure 7.3 Concentration response curves for the dihydropyridine calcium antagonist felodipine (FELO; upper graph) and agonist Bay K8644 (lower graph) in small arteries that were distended to either $0.7 \mathrm{D}_{0}$ (open symbols) or $1.3 \mathrm{D}_{0}$ (filled symbols). Concentration response curves were performed on precontractions induced by $125 \mathrm{mM}$ K-KRB (circles) or $10 \mu \mathrm{M}$ noradrenaline (triangles). Those for Bay K8644 were constructed in resting preparations. Data are mean \pm SEM $(n=8)$.

Figure 7.4 Concentration response curves for the potassium channel opener BRL-38227 (upper panel). Concentration response curves for potassium (lower left) and noradrenaline (lower right) in the absence (circles) or presence (triangles) of $3 \mu \mathrm{M}$ BRL-38227 in isolated small mesenteric arteries distended to either $0.7 \mathrm{D}_{0}$ (open symbols) or $1.3 \mathrm{D}_{0}$ (filled symbols). Concentration response curves for BRL-38227 were constructed on top of precontractions induced by $10 \mu \mathrm{M}$ noradrenaline. Shown are means \pm SEM as a fraction of the maximal response in the absence of BRL-38227.

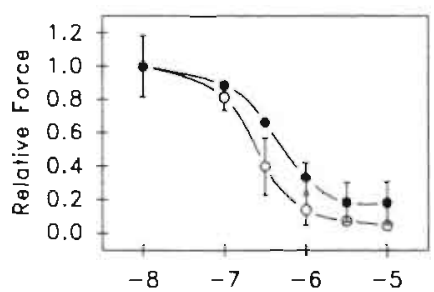

Log Molar [BRL-38227]

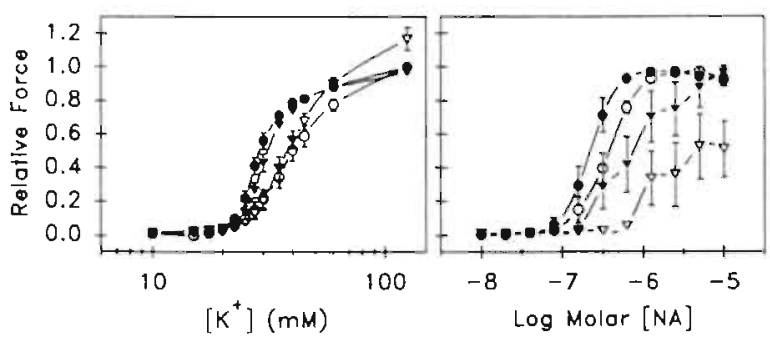


Table 7.2. Effects of the potassium channel opener BRL-38227 on hypersensitivity to potassium and noradrenaline in isolated mesenteric resistance arteries of WKY rats.

\begin{tabular}{|c|c|c|c|c|}
\hline & \multicolumn{2}{|c|}{ potassium } & \multicolumn{2}{|c|}{ noradrenaline } \\
\hline & $0.7 \mathrm{D}_{0}$ & $1.3 \mathrm{D}_{0}$ & $0.7 \mathrm{D}_{0}$ & $1.3 \mathrm{D}_{0}$ \\
\hline \multicolumn{5}{|l|}{$\mathrm{E}_{\max }(/$ control max $)$} \\
\hline+ BRL-38227 $1 \mu \mathrm{M}$ & $1.09 \pm 0.07(6)$ & $1.01 \pm 0.02(9)$ & $0.86 \pm 0.04^{b}(6)$ & $0.98 \pm 0.02(8)$ \\
\hline+ BRL-38227 $3 \mu \mathrm{M}$ & $1.17 \pm 0.07(6)$ & $0.97 \pm 0.02(8)$ & $0.54 \pm 0.19^{b}$ & $0.99 \pm 0.04^{\text {a }}(8)$ \\
\hline Sensitivity & \multicolumn{2}{|c|}{$\mathrm{EC}_{50}(\mathrm{mM})$} & \multicolumn{2}{|c|}{$\mathrm{pD}_{2}\left(-\log\right.$ Molar $\left.\left[\mathrm{EC}_{50}\right]\right)$} \\
\hline Control & $41.6 \pm 2.8(6)$ & $28.9 \pm 0.9^{2}(9)$ & $6.45 \pm 0.08(6)$ & $6.76 \pm 0.10$ \\
\hline$+\mathrm{BRL}-382271 \mu \mathrm{M}$ & $43.0 \pm 2.2(6)$ & $27.0 \pm 0.7^{\mathrm{a}}(9)$ & $5.73 \pm 0.09^{b}(6)$ & $6.06 \pm 0.17^{b}(8)$ \\
\hline$+\mathrm{BRL}-382273 \mu \mathrm{M}$ & $41.1 \pm 2.6(6)$ & $30.7 \pm 0.7^{a}(8)$ & $5.70 \pm 0.11^{b}(4)$ & $6.06 \pm 0.18^{b}(8)$ \\
\hline
\end{tabular}

Maximal contractile responses $\left(E_{\max }\right)$ and sensitivities for potassium $\left(E C_{50}\right)$ and noradrenaline $\left(\mathrm{pD}_{2}\right)$ in the absence (control) or presence of 1 or $3 \mu \mathrm{M}$ BRL-38227. Values are displayed as means \pm SEM. Number of observations between brackets. ${ }^{a}$ and ${ }^{b}$ indicate statistical significance of difference (ANOVA (Wallenstein, 1980), $P<0.05$ ) between low $(0.7$ $\left.D_{0}\right)$ and high $\left(1.3 D_{0}\right)$ distension or as compared to control, respectively.

Differences in sensitivity for contractile responses to potassium, but also noradrenaline can result from changes in membrane potential resulting from distension (Kirber et al., 1988; Johansson, 1989; Laher and Bevan, 1989). This would in turn affect the function of voltage operated calcium channels and hence result in higher $\left[\mathrm{Ca}^{2+}\right]_{i}$ with similar levels of stimulation. This hypothesis was addressed by evaluating the effect of hyperpolarising interventions on the distensiondependent hypersensitivity to vasoconstrictors. The relaxing effect of the potassium channel opener BRL-38227 was significantly reduced in vessels at $1.3 \mathrm{D}_{0}$ (Fig. 7.4) $\left(\mathrm{pD}_{2}\right.$ values for BRL-38227 averaged $6.14 \pm 0.18$ and $5.76 \pm 0.16$ at 0.7 $D_{0}$ and $1.3 D_{0}$, respectively). The sensitivity for noradrenaline was reduced equally in vessels at $1.3 \mathrm{D}_{0}$ and $0.7 \mathrm{D}_{0}$ by 1 and $3 \mu \mathrm{M}$ BRL-38227 (Table 7.2), whereas maximal contractile responses to $10 \mu \mathrm{M}$ noradrenaline were significantly reduced by BRL-38227 in vessels at $0.7 \mathrm{D}_{0}$ but not in those set at $1.3 \mathrm{D}_{0}$ (Fig. 7.4 and Table 7.2). Maximal contractile responses and sensitivities to potassium in vessels at either distension were not affected by BRL-38227 (Fig. 7.4 and Table 7.2). Returning potassium to vessels incubated in potassium deficient solution transiently reduced contractile responses to $10 \mu \mathrm{M}$ noradrenaline. The amplitude of this relaxation, which has been attributed to activation of the electrogenic sodium pump (De Mey and Vanhoutte, 1982; Hermsmeyer, 1982), tended to be larger at $0.7 \mathrm{D}_{0}$ than at $1.3 \mathrm{D}_{0}$ in both WKY and SHR. This did, however not reach statistical significance. These relaxing responses to potassium averaged (as a percentage of the response to $10 \mu \mathrm{M}$ noradrenaline): $50.7 \pm 11.6 \%(n=5)$ 
and $34.7 \pm 11.1 \%(n=5)$ in vessels of WKY and $57.0 \pm 7.9 \%(n=6)$ and $37.4 \pm$ $11.7 \%(n=6)$ in vessels of $S H R$ at $0.7 D_{0}$ and $1.3 D_{0}$, respectively.

\section{DISCUSSION}

We evaluated effects of distension of isolated mesenteric resistance-sized arteries on the reactivity and sensitivity to vasoconstrictor substances and calcium. The results indicate that distension increases the sensitivity for the contractile effects of potassium, noradrenaline and calcium in maximally depolarised vessels. Indirect pharmacological experiments suggest that distension could affect membrane potential and consequently the activity of voltage-operated calcium channels. Furthermore, distension may affect intracellular mechanisms controlling the calcium sensitivity of the contractile apparatus.

Vascular reactivity is mainly mediated through electromechanical and pharmacomechanical coupling. By opening of calcium channels in the cell membrane through changes in membrane potential or following receptor activation these mechanisms induce the influx of calcium and contraction (Somlyo and Somlyo, 1968; Van Breemen et al., 1986). However, some phenomena observed in vascular smooth muscle can not be explained by either of these pathways. Stretch of isolated small arteries has been shown to induce a transient contractile response which depended on the presence of extracellular calcium but could not be blocked by a dihydropyridine calcium antagonist (Hwa and Bevan, 1986a). Also in isolated smooth muscle cells it has been shown that stretching of the cell leads to a dihydropyridine-resistant increase in $\left[\mathrm{Ca}^{2+}\right]_{i}$ (Meininger et al., 1991). Stretch-operated calcium channels have therefore been proposed that differ from voltage operated calcium channels (Hwa and Bevan, 1986a; Laher and Bevan, 1989).

The contractile response that is observed due to stretch of vascular smooth muscle is, however, not maintained. Although a stretch induced contractile response has never been observed in our experimental setup with mesenteric resistance-sized arteries of the rat (unpublished observations), the present results show that the maintenance of the contractile response following maximal depolarisation was persistently affected by static distension (Fig. 7.1). Construction of a circumferential length-tension curve by stimulating isolated sympathectomised resistance arteries with high potassium at increasing diameters generates a relation between force and diameter (see Chapter 2) as is displayed in figure 7.1 for WKY and SHR vessels. Contractile responses to potassium consist of a biphasic response (Boonen and De Mey, 1990) of which the amplitude is length-dependent. This has previously been ascribed to effects of smooth muscle length on the overlap of contractile filaments (Mulvany and Warshaw, 1979; Harris and Warshaw, 1991). Besides effects on overlap of contractile filaments, distension significantly affected the relation between the initial transient and sustained response (Fig. 7.1 and Fig. 2.3). With increasing diameters, the 
ratio between sustained and transient contractile response to potassium $\left(\mathrm{K}_{\mathrm{s}} / \mathrm{K}_{\mathrm{t}}\right)$ increases in both WKY and SHR. However in SHR the ratio between sustained and transient response is increased (Fig. 7.1). On previous occasions (Boonen and De Mey, 1990) the relation between $K_{t}$ and $K_{s}$ was found to be differently affected by dihydropyridine calcium antagonists (see also Chapter 3 and 4). From these results we hypothesised that voltage operated calcium channels could reside in three different states and that the equilibrium between the activated (open) state and the inactivated (closed) state of the voltage operated calcium channel (see Chapter 4) was susceptible to modulation by noradrenaline. Whether this also applies for distension will be discussed bellow.

Nilsson and Sjöblom, (1985) have previously shown that maintained distension of isolated resistance arteries resulted in a persisting hypersensitivity to noradrenaline but not potassium. Price et al., (1981) on the other hand, showed a shift in sensitivity to both potassium and noradrenaline with increasing distension of isolated tibial arteries of the dog. The present results confirm the latter observations; a hypersensitivity not only to noradrenaline but also potassium and calcium is observed in vessels of WKY and SHR as a result of distension (Fig. 7.2 and Table 7.1). Different mechanisms may be responsible for these effects. The role of stretch-operated calcium channels is probably minor because as pointed out above, the recorded effects of distension do not result from the dynamic stretch but are maintained for time periods exceeding hours. It has been suggested that distension reduces the endothelial release of nitric oxide (Dainty et al., 1990; Lamontagne et al., 1992). The hypersensitivity to potassium we observed at $1.3 \mathrm{D}_{0}$ was, however, unaffected by removal of the endothelium (Table 7.1).

The shift in sensitivity to extracellular calcium in vessels that were maximally depolarised (Fig. 7.2) suggests that distension may affect the relation between membrane potential and the probability of voltage operated calcium channels to reside in an opened state (Nelson et al., 1990). However, the lack of differences in the inhibitory effect of the dihydropyridine calcium antagonist felodipine on maximal contractile responses to potassium or noradrenaline at high and low distension suggests that at least the affinity of voltage-operated calcium channels for the dihydropyridine is not affected by distension (Fig. 7.3). Yet, the dihydropyridine calcium agonist Bay K8644 induced contractile responses only in vessels at $1.3 \mathrm{D}_{0}$ (Fig. 7.3). Bay K8644 has been shown to shift the activation curve of voltage operated calcium channels towards more negative membrane potentials (Bean et al., 1986; Nelson et al., 1990). Whether the unchanged affinity for felodipine at high and low distension has any predicting value for the relation between membrane potential and calcium channel function is debatable. Together with the observed effects of Bay K8644 at high and low distension, however, these results may suggest that either the activation curve for voltage operated calcium channels is shifted by distension or distension affects membrane potential.

Membrane potential in arterial smooth muscle is mainly governed by the action of the electrogenic sodium pump, different potassium channels and by the permeability of the membrane for different cations and anions (Hermsmeyer, 
1982; Nelson et al., 1990). Omitting potassium from the extracellular medium inactivates the electrogenic sodium pump resulting in intracellular sodium gain (De Mey and Vanhoutte, 1982; Hermsmeyer, 1982). Returning potassium to the extracellular solution then results in a transient hyperactivity of the sodium-potassium ATPase, leading to a transient hyperpolarisation of the vascular smooth muscle cell and hence a transient relaxing response (De Mey and Vanhoutte, 1982; Hermsmeyer, 1982). The present results show that the potassium-induced relaxation is reduced in vessels at $1.3 \mathrm{D}_{0}$ as compared to $0.7 \mathrm{D}_{0}$, suggesting that i) the activity of the sodium-pump is affected ii) sodium influx is modified or iii) membrane potential is less polarised at $1.3 \mathrm{D}_{0}$ than at $0.7 \mathrm{D}_{0}$. The shift to the right in sensitivity of the relaxing effect of the potassium channel opener BRL-38227 at 1.3 $D_{n}$ (Fig 7.4) is in agreement with the latter hypothesis. However, the precontraction induced by noradrenaline may have been affected by distension. Therefore, full concentration-response curves for potassium and noradrenaline were repeated in the presence of increasing concentrations of BRL-38227. As shown in figure 7.4 and Table 7.2, BRL-38227 hardly affected contractile responses to potassium as would be expected for a potassium channel opener (Cook et al., 1988). If anything, only stimulations with intermediate potassium concentrations would be susceptible to inhibition (Cook et al., 1988). On the other hand, BRL-38227 had marked effects on responses to noradrenaline. Maximal contractile responses to noradrenaline at $0.7 \mathrm{D}_{0}$ but not $1.3 \mathrm{D}_{0}$ were reduced already by $1 \mu \mathrm{M}$ BRL-38227. The sensitivity for noradrenaline was affected equally at both distensions by BRL-38227.

Taken together, the reduced sensitivity to BRL-38227 and reduced potassium induced relaxations indicate that membrane potential could be less polarised at high distension compared to low distension. The depolarisation of the membrane potential may consequently result in an increased influx of calcium with intermediate stimulations of the vessel preparations and a shift in the sensitivity for vasoconstrictors. Additionally the sensitivity to extracellular calcium in vessels that were maximally depolarised was increased in vessels at high distension. This effect can not be explained by differences in membrane potential because high potassium solution maximally depolarises vascular smooth muscle (Mulvany et al., 1982). Remains the possibility that an intracellular mechanism involved in the sensitisation of the contractile apparatus for calcium is affected by distension. Nilsson and Sjöblom, (1985) suggested that an intracellular mechanism was probably responsible for the altered distensiondependent sensitivity for noradrenaline. This catecholamine has previously been described to increase the sensitivity of the contractile apparatus in vascular smooth muscle for calcium (Himpens et al., 1990; Nishimura et al., 1990; Jensen et al., 1992). Protein kinase-C has been suggested to be responsible for this effect (Drenth et al, 1989; Nishimura et al., 1990). Whether the calcium sensitising effect of distension is also mediated through effects on the activity of protein kinase-C remains to be established. Yet, protein kinase- $C$ has been shown to be translocated and activated by deformation of striated muscle cells (Richert et al., 1987). Furthermore, in stretch-induced and pressure-induced myogenic responses, protein kinase-C activity may play an important role (Laher et al., 1989; Osol et al., 
1991). Additionally, protein kinase- $C$ has been shown to affect the function of voltage operated calcium channels (Fish et al., 1988; Boonen and De Mey, 1991). In this regard, the differences that were observed in the ratio of sustained and transient contractile response to potassium during construction of a diameter-tension curve and the upregulation of the ratio between these contractile phases in small vessels of SHR, may involve differences in activation of protein kinase-C (Uehara et al., 1988).

In conclusion, it was shown that distension of isolated mesenteric small arteries affected the sensitivity and reactivity to vasoconstrictors and calcium. This phenomenon may involve effects of distension on the cellular regulation of membrane potential and consequently on the activity of voltage operated calcium channels. Additionally, distension may affect intracellular mechanisms that control the contractile response. The nature of these changes, however, remain unknown but may involve modulatory effects of distension on the activity of second messengers and ion channels. 


\section{Distension Sensitises Small Artery Smooth Muscle to Vasoconstrictors and to Intracellular Calcium}

Harrie C.M. Boonen, Peter E. Jensen ${ }^{\mathrm{a}}$, Michael J. Mulvany ${ }^{\mathrm{a}}$, Christian Aalkjaer, and Jo G.R. De Mey

Departments of pharmacology, University of Limburg, Maastricht, the Netherlands, and ${ }^{3}$ University of Aarhus, Århus, Denmark. 


\section{SUMMARY}

Effects of distension on sensitivity to vasoconstrictors may contribute to myogenic autoregulatory responses in small arteries. We evaluated the role of calcium and membrane potential $\left(V_{m}\right)$ herein. Small arteries were isolated from the mesentery of the rat, sympathectomised and mounted for recording of isometric force development, intracellular calcium concentration $\left(\left[\mathrm{Ca}^{2+}\right]_{\mathrm{i}}\right)$ and $\mathrm{V}_{\mathrm{m}}$. Vessels were set at 0.7 or 1.3 times the diameter $\left(\mathrm{D}_{0}\right)$ at which they contracted maximally in response to $125 \mathrm{mM}$ potassium $\left(\mathrm{K}^{+}\right)$. $\left[\mathrm{Ca}^{2+}\right]$, was measured simultaneously with force using Fura-2. At $1.3 \mathrm{D}_{0}$, compared to 0.7 $D_{0}$, i) vessels were hypersensitive to the contractile effects of $\mathrm{K}^{+}$and noradrenaline (NA),

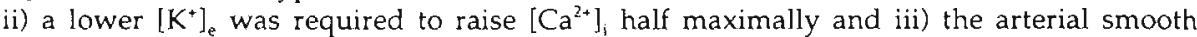
muscle cells were less polarised under basal conditions and in the presence of intermediate $\left[\mathrm{K}^{+}\right]_{\mathrm{e}}$ but not in the presence of NA. NA and $\mathrm{K}^{+}$resulted in similar maximal $\left[\mathrm{Ca}^{2+}\right]_{i}$ at the different distensions. Yet at $0.7 \mathrm{D}_{0}, \mathrm{~K}^{+}$yielded less maximal force than at 1.3 $D_{f 1}$. In the presence of $\mathrm{NA}$, the sensitivity of the contractile machinery to $\left[\mathrm{Ca}^{2+}\right]_{\text {, was }}$ higher at $1.3 \mathrm{D}_{0}$ than $0.7 \mathrm{D}_{0}$. These results confirm that the sensitivity for vasoconstrictors in small artery smooth muscle is increased by distension. Changes in $\mathrm{V}_{\mathrm{m}}$ and modulation of the relation between active force and $\left[\mathrm{Ca}^{2+}\right]_{1}$ are involved herein.

\section{INTRODUCTION}

Two major factors control the diameter of blood vessels that are involved in regulation of local blood flow and total peripheral resistance. Vascular structure participates in long term control while arterial tone regulates blood flow in a more flexible acute way. Both factors can be modulated by a variety of processes. Homeostasis is achieved by the interaction of several regulating variables including neural (Miller, 1991) and humoral agents (Vanhoutte et al., 1981), the endothelium (Griffith and Edwards, 1990; Rubanyi et al., 1990), mechanical factors (Faber and Meininger, 1990), tissue metabolites (McGillivray-Anderson and Faber, 1990) and myogenic activity (Johnson, 1980).

Myogenic responses can be elicited in isolated resistance-vessel segments or perfused vascular beds by distension or increases in perfusion pressure (Hwa and Bevan, 1986; Rubanyi et al., 1990). It has previously been shown that the sensitivity for both ionic and pharmacological vasoconstrictors increases with increasing distension or conversely, decreases at low distensions of an isolated vessel (Price et al., 1981; Nilsson and Sjöblom, 1985). This observation could be of relevance for the initiation and negative feedback control of the myogenic autoregulatory response. The underlying mechanisms responsible for the change in sensitivity for vasoactive stimuli, however, remain largely unknown. It has been suggested that membrane potential $\left(V_{n}\right)$ and sarcolemmal calcium channels may play a role in stretch induced or pressure induced responses (Laher and Bevan, 1989; Nordlander, 1989; Nelson et al., 1990). Activation of second messenger systems could also be involved (Laher and Bevan, 1987; Hill et al., 1990; Osol et al., 1991). 
Stretch has furthermore been shown to affect myosin light chain kinase activity (Barany et al., 1990).

The objective of the present study was to investigate whether distension of small mesenteric arteries influences membrane potential and intracellular calcium concentration. We therefore recorded mechanical responses, intracellular calcium concentration $\left(\left[\mathrm{Ca}^{2+}\right]_{i}\right)$ and $V_{m}$ in isolated sympathectomised mesenteric small arteries of the rat exposed in vitro to a low or to a high level of distension. The results suggest a role for $V_{m}$ and for changes in the effect of $\left[\mathrm{Ca}^{2+}\right]_{i}$ on force.

\section{MATERIAL AND METHODS}

\section{Vessel preparation}

Experiments were performed in small arteries that had been isolated from the mesentery of 14 week old male Wistar rats (locally inbred strain, Århus, Denmark). Rats were killed by a sharp blow on the head and cervical dislocation. The mesentery was isolated and immersed in a physiological salt solution. Third to fourth order side branches of the superior mesenteric artery $(\emptyset \approx 200 \mu \mathrm{m})$ were then dissected from the mesentery. To exclude influences of sympathetic nerve endings in the vessel segments (Vanhoutte et al., 1981), the vessels were chemically sympathectomised by $10 \mathrm{~min}$ incubation at $37^{\circ} \mathrm{C}$ in a bicarbonate free Krebs solution that contained $300 \mu \mathrm{g} / \mathrm{ml}$ 6-hydroxydopamine (Aprigliano and Hermsmeyer, 1977). The vessel segments (axial length approximately $2 \mathrm{~mm}$ ) were then mounted on two stainless steel wires $(\varnothing 40 \mu \mathrm{m})$ as ring segments in an isometric myograph between a displacement device and a force transducer (Kistler Morse DSC6, Seattle, USA) according to the method described by Mulvany and Halpern (Mulvany and Halpern, 1977). Vessel segments were subsequently stretched to their individual optimal lumen diameter $\left(D_{0}\right)$ at which the initial transient contractile response to $125 \mathrm{mM}$ potassium was maximal as was previously described (De Mey and Brutsaert, 1984; Boonen and De Mey, 1990). During isolation, mounting and experimentation, the vessels were immersed in Krebs Ringer bicarbonate buffered solution (KRB); composition (mM): $\mathrm{NaCl}$ 118.5; $\mathrm{KCl} 4.7 ; \mathrm{MgSO}_{4} \cdot 7 \mathrm{H}_{2} \mathrm{O} 1.2 ; \mathrm{KH}_{2} \mathrm{PO}_{4}$ 1.2; $\mathrm{NaHCO}_{3} 25.0 ; \mathrm{CaCl}_{2} \cdot 2 \mathrm{H}_{2} \mathrm{O} 2.5$; glucose 5.5, EDTA 0.026, that was continuously aerated with $95 \% \mathrm{O}_{2}$ and $5 \%$ $\mathrm{CO}_{2}$. High potassium Krebs (K-KRB) was identical to KRB except that all the $\mathrm{NaCl}$ was replaced by an equimolar amount of $\mathrm{KCl}$. Intermediate extracellular potassium concentrations were obtained by mixing appropriate volumes of KRB and K-KRB. Stimulations with noradrenaline were always performed in the presence of $1 \mu \mathrm{M}$ of the $\beta$-adrenergic blocking agent propranolol. 


\section{Effects of distension on membrane potential}

After determination of $\mathrm{D}_{0}$, the vessels were set at diameters that were either $30 \%$ less or $30 \%$ larger than $\mathrm{D}_{0}$ ie. $0.7 \mathrm{D}_{0}$ and $1.3 \mathrm{D}_{0}$, respectively. Then, membrane potential measurements were performed using an impaling glass microelectrode as was described by Mulvany and coworkers (Mulvany et al., 1982). $\mathrm{V}_{\mathrm{m}}$ was measured at rest $(5.9 \mathrm{mM}$ potassium ) and in vessels that were stimulated with intermediate concentrations of potassium ( 25 and $35 \mathrm{mM}$ ) or noradrenaline $(0.3$ and $3 \mu \mathrm{M})$.

\section{Effects of distension on calcium handling}

To analyse effects of distension on $\left[\mathrm{Ca}^{2+}\right]_{i}$, we measured in separate sets of experiments $\left[\mathrm{Ca}^{2+}\right]_{\mathrm{i}}$ using the fluorescent calcium indicator Fura-2. Simultaneous measurements of force and $\left[\mathrm{Ca}^{2+}\right]_{i}$ were performed as described by Jensen et al., (1992). Sympathectomised vessels were mounted in a myograph and loaded for 4 hours at room temperature in a KRB solution that contained $10 \mu \mathrm{M}$ of Fura-2-AM (ester of Fura-2), $0.1 \%$ cremophor EL, 0.5\% dimethyl sulfoxamine (DMSO) and $0.02 \%$ pluronic F127. Following loading of the dye, the vessels were stretched randomly to $0.7 \mathrm{D}_{0}$ or $1.3 \mathrm{D}_{0}$ and concentration response curves for potassium and noradrenaline were obtained at each distension. The myograph was placed on an inverted microscope (Zeiss, axiovert 10), and the emission at $340 \pm 5 \mathrm{~nm}$ and $380 \pm 5 \mathrm{~nm}$ excitation was measured through a $500-530 \mathrm{~nm}$ filter using a photomultiplier. The emission signals and force were measured at 10 second intervals and stored on an IBM personal computer during experimentation. Calibration of emission signals, using the equation: $\left[C a^{2 \cdot}\right]_{i}=K_{d} \cdot \beta \cdot\left(R-R_{\min }\right) /\left(R_{\max }-R\right)$, implied determination of minimal $\left(R_{\min }\right)$ and maximal $\left(R_{\max }\right)$ ratios of emission signals at 340 and $380 \mathrm{~nm}$ excitation, both corrected for emission signals remaining at the respective excitation wavelengths after quenching the Fura-2 fluorescence with $\mathrm{Mn}^{2+} . \mathrm{R}_{\min }$ was measured in calcium free HEPES buffered KRB with addition of $20 \mu \mathrm{M}$ of the calcium ionophore ionomycin, whereas $R_{\max }$ was subsequently measured in HEPES buffered KRB containing $5 \mathrm{mM}$ calcium. $\beta$ refers to the ratio between emission signals at $380 \mathrm{~nm}$ excitation under calcium free and under calcium saturated conditions, both corrected for signals remaining after eliminating the fluorescence of Fura- 2 with $\mathrm{Mn}^{2+}$. The dissociation constant $\left(\mathrm{K}_{\mathrm{d}}\right)$ for Fura-2 at $37^{\circ} \mathrm{C}$ was taken as $224 \mathrm{nM}$ (Grynkiewicz et al., 1985). Following measurements of the 340 $\mathrm{nm}$ and $380 \mathrm{~nm}$ emission signals for the calculation of $R_{\min }$ and $R_{\max }$, the emission signals remaining at the respective excitation wavelengths after addition of $8 \mathrm{mM} \mathrm{Mn}{ }^{2+}$ were measured. Emission signals remaining after quenching the fluorescence with $\mathrm{Mn}^{2+}$ were measured at 0.7 and $1.3 \mathrm{D}_{0}$, and were used for subtractions at the respective distension. The solutions that were used to determine $R_{\text {nax }}$ and $R_{\min }$ had the following composition (mM): HEPES 10.0; $\mathrm{KCl}$ 144.6; $\mathrm{MgCl}_{2} \cdot 7 \mathrm{H}_{2} \mathrm{O}$ 1.17; glucose 6.0 and either $5 \mathrm{mM} \mathrm{CaCl}{ }_{2} \cdot 2 \mathrm{H}_{2} \mathrm{O}$ or $2 \mathrm{mM}$ EGTA, as indicated. 


\section{Drugs}

6-hydroxydopamine $\mathrm{HCl}$, noradrenaline $\mathrm{HCl}$ (l-arterenol bitartrate), dlpropranolol $\mathrm{HCl}$, EDTA, DMSO (dimethyl sulfoxamine), cremophor EL, ionomycin and HEPES were purchased from Sigma Chemicals Co. (Saint Louis, MO, USA). Fura-2 AM and pluronic F127, were obtained from Molecular Probes Inc. (Junction City, OR, USA).

\section{Statistics}

For both potassium and noradrenaline, concentration response curves were constructed simultaneously with respect to increases in $\left[\mathrm{Ca}^{2+}\right]_{i}$ and active force. Sensitivities of either effect for vasoconstrictors was determined from the concentration response curves using a least squares sigmoidal curve fit (Graphpad Software, San Diego, CA, USA). Differences between observations at the two distensions with respect to maximal effects and sensitivities were evaluated by a two-way analysis of variance followed by a t-test as described by Bonferroni (Wallenstein et al., 1980). Statistical analysis was performed using the Crunch Interactive Statistical Package (CRUNCH, Software Corp., San Francis$\mathrm{CO}, \mathrm{CA}, \mathrm{USA}$ ).

\section{RESULTS}

\section{Effects of distension on electro-mechanical coupling}

Under resting conditions $\left(5.9 \mathrm{mM} \mathrm{K} \mathrm{K}^{+}\right),\left[\mathrm{Ca}^{2+}\right]_{\mathrm{i}}$ did not differ between vessels set at 0.7 or $1.3 \mathrm{D}_{0}$ (Fig. 8.1.). Raising $\left[\mathrm{K}^{+}\right]_{\mathrm{E}}$ caused concentration-dependent increases in $\left[\mathrm{Ca}^{2+}\right]_{i}$. Intermediate $\left[\mathrm{K}^{+}\right]_{\mathrm{e}}$ elevated $\left[\mathrm{Ca}^{2+}\right]_{\mathrm{i}}$ to a larger extent in vessels set at $1.3 \mathrm{D}_{0}$ than in those maintained at $0.7 \mathrm{D}_{0}$ (Fig. 8.1). Yet, maximal $\left[\mathrm{Ca}^{2+}\right]_{1}$ observed in the presence of $125 \mathrm{mM}$ potassium was comparable at 0.7 $\mathrm{D}_{0}$ and $1.3 \mathrm{D}_{0}$ (Table 8.1). Vessels at $1.3 \mathrm{D}_{0}$ were also hypersensitive to the contractile effect of elevated $\left[\mathrm{K}^{+}\right]_{\mathrm{e}}$ (Fig. 8.1 and Table 8.1). They, furthermore, contracted more vigorously in response to $125 \mathrm{mM}$ potassium than those at $0.7 \mathrm{D}_{0}$ despite comparable elevation of $\left[\mathrm{Ca}^{2+}\right]_{i}$ (Fig. 8.1 and Table 8.1). Effects of distension on sensitivity for the calcium raising and contractile effect of $\left[\mathrm{K}^{+}\right]_{e}$ may be due to a depolarising influence. Figure 8.2 shows $\mathrm{V}_{\mathrm{m}}$ in vessels that were set at different distension and exposed to $5.9,25$ or $35 \mathrm{mM}$ potassium. Small artery smooth muscle was significantly less polarised at 1.3 than at $0.7 D_{0}$ in resting conditions. This difference in $\mathrm{V}_{\mathrm{m}}$ persisted in the presence of 25 and $35 \mathrm{mM}$ potassium. 


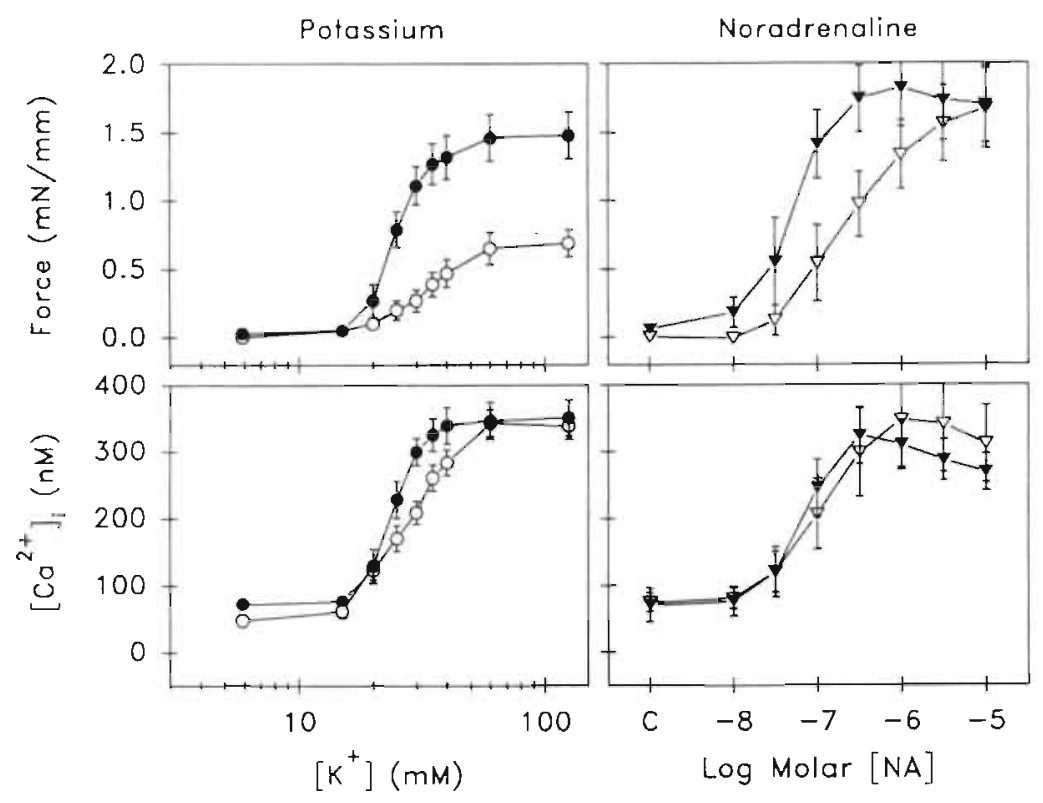

Figure 8.1. Force development (upper panels) and intracellular calcium concentrations (lower pancls) measured simultaneously in isolated sympathectomised small arteries during construction of concentration-response curves for potassium (circles; left panels) and noradrenaline (triangles; right panels). The concentration-response curves were constructed at either $0.7 D_{0}$ (open symbols) or $1.3 \mathrm{D}_{0}$ (closed symbols). Shown are means $\pm \operatorname{SEM}\left(\mathrm{n}=11\right.$ and $\mathrm{n}=6$, for $\mathrm{K}^{+}$and $\mathrm{NA}$, respectively).

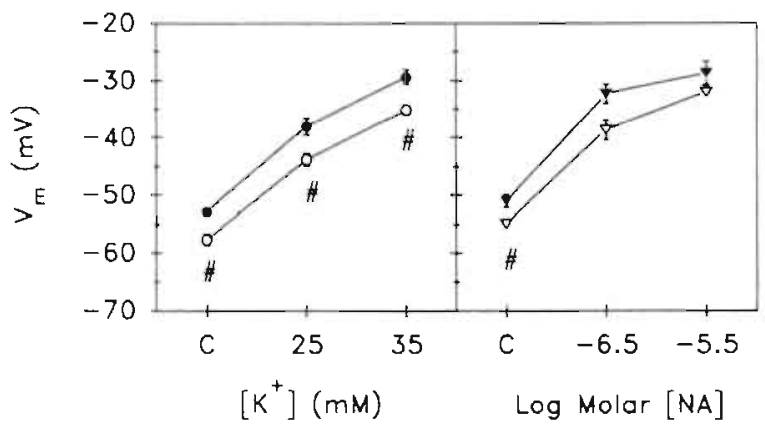

Figure 8.2. Graph showing membrane potential $\left(V_{m}\right)$ in vascular smooth muscle cells. $V_{m}$ was assessed directly using a glass microelectrode in vessels that were distended to either $0.7 \mathrm{D}_{0}$ (open symbols) or $1.3 \mathrm{D}_{0}$ (closed symbols). Membrane potential was measured in resting preparations ( $C$ ) and in vessels that were stimulated with 25 and $35 \mathrm{mM}$ potassium (circles) $(n=8-11)$ or 0.3 and $3 \mu \mathrm{M}$ noradrenaline (triangles) $(n=7-9)$. Displayed are means \pm SEM. \# indicates statistical significance of differences between vessels at high and low distension (unpaired t-test, $\mathrm{P}<0.05$ ). 
Table 8.1. Effects of potassium and noradrenaline on intracellular calcium and active wall tension in mesenteric resistance arteries at low and high levels of distension".

$0.7 \mathrm{D}_{0} \quad 1.3 \mathrm{D}_{0} \quad \mathrm{n}$

Potassium

\begin{tabular}{|c|c|c|c|}
\hline AWT $E_{\max }(\mathrm{mN} / \mathrm{mm})$ & $0.67 \pm 0.11$ & $1.38 \pm 0.16^{\mathrm{a}}$ & 11 \\
\hline $\mathrm{EC}_{\mathrm{so}_{0}}(\mathrm{mM})$ & $35.6 \pm 1.7$ & $24.1 \pm 1.2^{\mathrm{a}}$ & 11 \\
\hline$\left[\mathrm{Ca}^{2+}\right], \mathrm{E}_{\max }(\mathrm{nM})$ & $343 \pm 19$ & $345 \pm 25$ & 11 \\
\hline $\mathrm{EC}_{s_{0}}(\mathrm{mM})$ & $27.5 \pm 1.2$ & $22.9 \pm 1.0^{\mathrm{a}}$ & 11 \\
\hline \multicolumn{4}{|l|}{ Noradrenaline } \\
\hline AWT $E_{\max }(\mathrm{mN} / \mathrm{mm})$ & $1.62 \pm 0.29$ & $1.69 \pm 0.25$ & 6 \\
\hline $\mathrm{pD}_{2}$ & $6.85 \pm 0.21$ & $7.43 \pm 0.17^{\mathrm{a}}$ & 6 \\
\hline$\left[\mathrm{Ca}^{2+}\right], \mathrm{E}_{\max }(\mathrm{nM})$ & $312 \pm 43$ & $317 \pm 40$ & 6 \\
\hline $\mathrm{pD}_{2}$ & $7.12 \pm 0.16$ & $7.43 \pm 0.20$ & 6 \\
\hline
\end{tabular}

Active wall tension (AWT) and intracellular calcium concentrations $\left(\left[\mathrm{Ca}^{2+}\right]_{\mathrm{i}}\right)$ were measured simultaneously. Displayed are mean values \pm SEM of maximal AWT $(\mathrm{mN} / \mathrm{mm})$ and maximal $\left[\mathrm{Ca}^{2+}\right],(\mathrm{nM})$ along with sensitivities for potassium $\left(\mathrm{EC}_{50}\right)$ and noradrenaline $\left(\mathrm{pD}_{2}=-\log \left[E C_{50}\right]\right)$ with respect to both effects. Sympathectomised vessels were first set randomly at either 0.7 or $1.3 \mathrm{D}_{0}$ and tested thereafter at 1.3 or $0.7 \mathrm{D}_{0} . \mathrm{n}$; number of observations. ", difference between 0.7 and $1.3 \mathrm{D}_{0}$ is statistically significant ( $\mathrm{p}<0.05$, analysis of variance followed by $\mathrm{t}$-test according to Bonferroni (Wallenstein 1980)). Experiments with noradrenaline were performed in the presence of $1 \mu \mathrm{M}$ propranolol.

\section{Effects of distension on pharmaco-mechanical coupling}

Contractile responses induced by noradrenaline were also affected by distension (Fig. 8.1). At $1.3 \mathrm{D}_{0}$ compared to $0.7 \mathrm{D}_{0}$, vessels were hypersensitive to the contractile effect of noradrenaline (Fig. 8.1, Table 8.1). Maximal force development induced by noradrenaline was, in contrast to potassium, similar at $0.7 \mathrm{D}_{0}$ and $1.3 \mathrm{D}_{0}$ (Fig. 8.1; 8.3 and Table 8.1). The difference in sensitivity for the contractile response of noradrenaline at the different distensions was not accompanied by a significant hypersensitivity to noradrenaline with respect to increase of $\left[\mathrm{Ca}^{2+}\right]_{i}$ (Table 8.1). The difference in $\mathrm{V}_{\mathrm{m}}$ that existed at rest between vessels at $0.7 \mathrm{D}_{0}$ and $1.3 \mathrm{D}_{0}$, was not statistically significant in the presence of 0.3 and $3 \mu \mathrm{M}$ noradrenaline (Fig 8.2). As for potassium, the maximal $\left[\mathrm{Ca}^{2+}\right]_{\mathrm{i}}$ obtained with noradrenaline was similar at $0.7 \mathrm{D}_{0}$ and $1.3 \mathrm{D}_{0}$ (Fig. 8.1 and Table 8.1). At any distension, $10 \mu \mathrm{M}$ noradrenaline induced a larger force development than $125 \mathrm{mM}$ potassium despite a similar increase in $\left[\mathrm{Ca}^{2+}\right]_{i}$ (Fig. 8.3). This is consistent with the conclusion by Himpens et al., (1990;) Nishimura 


\section{CHAPTER 8}

et al., (1990) and Jensen et al., (1992) that noradrenaline increases the sensitivity of the contractile machinery of vascular smooth muscle for $\left[\mathrm{Ca}^{2+}\right]_{i}$. However, distension increased the sensitivity of the contractile apparatus in the presence of noradrenaline with respect to $\left[\mathrm{Ca}^{2+}\right]_{i}$ even further (Fig. 8.3).

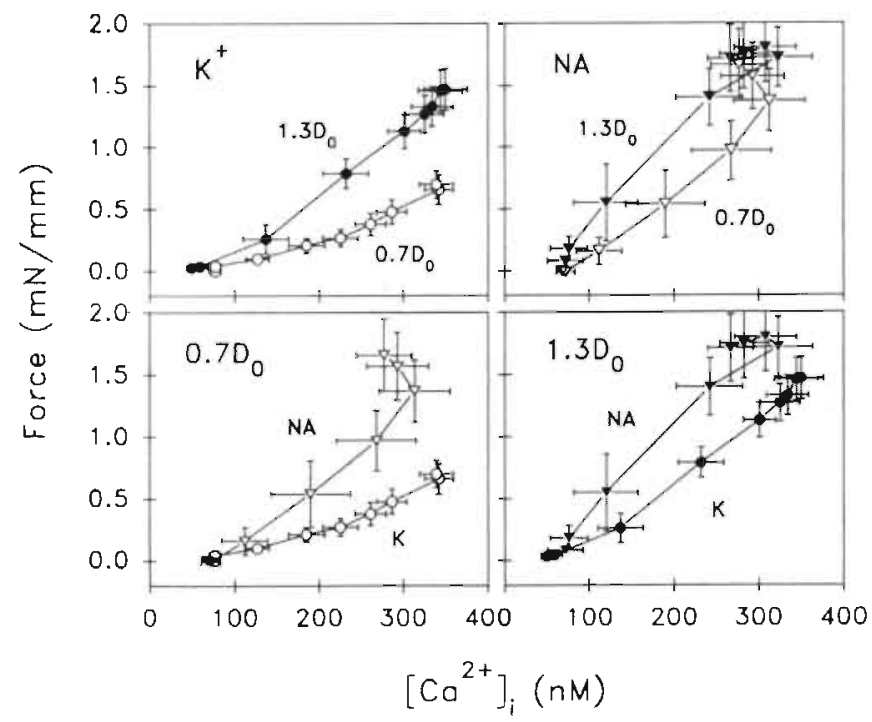

Figure 8.3. Figure showing the relationship between intracellular calcium concentrations $\left(\left|\mathrm{Ca}^{2+}\right|_{1}\right)$ and force development in sympathectomised small mesenteric arteries that were set at either $0.7 D_{0}$ (open symbols) or $1.3 D_{0}$ (closed symbols). Data are means \pm SEM ( $n=$ 6-11) of steady state responses to increasing concentrations of either potassium (circles) or noradrenaline (triangles). Note: upper and lower panels show similar results but these are arranged differently.

\section{DISCUSSION}

Increasing the diameter of small arteries had complex effects on excitation-contraction coupling in vascular smooth muscle. Responses to high potassium and noradrenaline were affected differently. This, along with measurements of $V_{m}$ and $\left[\mathrm{Ca}^{2+}\right]_{1}$ suggest that distension of small artery smooth muscle affects, besides the excitability of the cell membrane and the overlap between contractile filaments, the effectiveness of $\left[\mathrm{Ca}^{2+}\right]_{i}$ in producing active force.

Contractile reactivity of vascular smooth muscle is influenced by a broad variety of chemicals of neural, humoral or local origin and by physical factors such as strain, wall tension and shear stress. The latter have been suggested to be involved in myogenic responses (Johnson, 1980; Tesfamariam and Halpern, 1987; Rubanyi et al., 1990; Bevan and Laher, 1991). Together with a modulating role for neuro- 
transmitters (Faber and Meininger, 1990; Meininger and Faber, 1991) these are involved in autoregulation of local blood flow and maintenance of total peripheral resistance. It is not clear yet where and how physical factors are sensed within the vascular wall and how they are transduced into a change in contractility. It has been shown that dynamic stretch and distension of isolated vascular smooth muscle affects its reactivity to vasoconstrictors (Price et al., 1981; Nilsson and Sjoblom, 1985). The subject of this study was to evaluate which mechanisms may be involved in distension-induced alterations in vascular reactivity. The experiments were performed in vessels that are small enough to control local blood flow and total peripheral resistance. To allow smooth muscle length to be the externally controlled variable during in vitro experimentation, we deliberately studied the isolated preparations in an isometric setup in which vessel diameter could be set and maintained at individually defined levels.

Changes of the contractile response of muscle with distension is classically attributed to variation in the overlap between the contractile filaments (Gordon et al., 1966; Mulvany and Warshaw, 1979). The present results show, however, that the shape of the force-distension curves differ between potassium and noradrenaline activation. Although the responses to potassium and noradrenaline were similar at $1.0 \mathrm{D}_{0}$ and $1.3 \mathrm{D}_{0}$, the response to potassium was substantially less than the response to noradrenaline at $0.7 \mathrm{D}_{0}$. Therefore it appears that, depending on the type of activation, factors other than overlap between actin and myosin may contribute to changes in active force with distension. The present results do not allow us to determine whether these additional factors apply to the potassium or the noradrenaline activations.

In addition to altering resting wall tension and active force generation, distension altered the sensitivity for depolarising and pharmacological stimuli (Table 8.1). Depolarising stimuli induce contraction of vascular smooth muscle through opening of voltage operated calcium channels (Nelson et al., 1990). These channels open as a function of membrane potential (Nelson et al., 1990). Therefore, alterations in sensitivity for depolarising stimulation could be due to a change in membrane potential induced by distension, or a shift in the activation curve of voltage operated calcium channels. Increases in the length of isolated smooth muscle preparations and pressurisation of isolated large and small blood vessels (Smeda and Daniel, 1988; Laher and Bevan, 1989) have been observed to induce depolarisation. The underlying ionic mechanism has not been fully established yet. It has been proposed, however, that the depolarisation induced by the length change could be sufficient to activate voltage operated calcium channels and stimulate calcium entry (Nelson et al., 1990).

The role of membrane potential in the distension-dependent changes in sensitivity for vasoactive stimuli was therefore assessed directly. Plotting the membrane potential data against the corresponding values of $\left[\mathrm{Ca}^{2+}\right]_{i}$ in the presence of 5.9, 25 and $35 \mathrm{mM}$ shows a linear correlation (Fig. 8.4), which is not different between vessels at $0.7 \mathrm{D}_{0}$ and $1.3 \mathrm{D}_{0}$, suggesting that distension does not affect the relationship between membrane potential and $\left[\mathrm{Ca}^{2+}\right]_{i}$. The depolarisation induced by distension of relaxed vessels was not accompanied by a significant change in $\left[\mathrm{Ca}^{2+}\right]_{i}$ (Fig, 8.4) suggesting that within this range (-60 
to $-50 \mathrm{mV}$ ) of the membrane potential, calcium influx through voltage operated calcium channels is not significantly contributing to $\left[\mathrm{Ca}^{2+}\right]_{\text {i }}$ (Nelson et al., 1990) or that calcium influx through these channels is masked by counteracting mechanisms such as the calcium buffering capacity of the sarcoplasmic reticulum (Van Breemen and Saida, 1989). However, at intermediate potassium concentrations the more positive membrane potential at $1.3 \mathrm{D}_{0}$ was associated with an increased $\left[\mathrm{Ca}^{2+}\right]_{i}$ (Fig. 8.4), consistent with voltage operated calcium channels being voltage sensitive between -45 to $-30 \mathrm{mV}$ (Nelson et al., 1990). With respect to maximal force development, the increase in force for the same $\left[\mathrm{Ca}^{2+}\right]_{i}$ would be consistent with a more optimal overlap of contractile filaments at $1.3 \mathrm{D}_{0}$ than at 0.7 $\mathrm{D}_{0}$. Since it was not possible to obtain a supramaximal $\left[\mathrm{Ca}^{2+}\right]_{i}$ for force development, it was not possible to assess whether the sensitivity to $\left[\mathrm{Ca}^{2+}\right]_{i}$ was affected by distension.

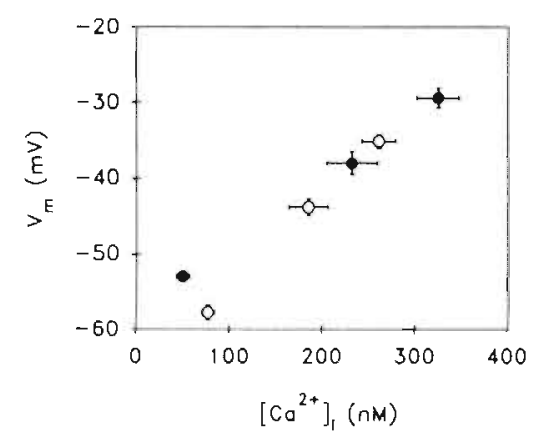

As discussed above, the higher force during activation with potassium at $1.3 \mathrm{D}_{0}$ compared to $0.7 \mathrm{D}_{0}$, despite a similar $\left[\mathrm{Ca}^{2+}\right]_{\mathrm{i}}$ would be consistent with a more optimal overlap of the contractile filaments at $1.3 \mathrm{D}_{0}$. However, during activation with noradrenaline, the maximal force (and $\left[\mathrm{Ca}^{2+}\right]_{i}$ ) was similar at 1.3 $D_{0}$ and $0.7 D_{0}$. This did not reflect an inability of the arteries to produce more force since at $1.0 D_{0}$ more force was produced with noradrenaline (Data not shown). Since the effect of changes in filament overlap on potassium and noradrenaline induced force is expected to be similar, these observations suggest that some mechanism in addition to overlap affects the relation between $\left[\mathrm{Ca}^{2+}\right]_{i}$ and force at different distensions. This mechanism is differently affected by noradrenaline and depolarisation with potassium. In this connection it is of interest that in a microcirculatory preparation, noradrenaline but not potassium enhanced the myogenic response to pressure increases (Faber and Meininger, 1990; Meininger and Faber, 1991).

During stimulation with noradrenaline, a hypersensitivity for the contractile effect of the $\alpha$-adrenergic agonist could be observed at high distension. Part of this hypersensitivity to noradrenaline may be explained by depolarisation induced increases in $\left[\mathrm{Ca}^{2+}\right]_{i}$ at high distension. However, the $\left[\mathrm{Ca}^{2+}\right]_{i}$ - force curve was shifted to the left at $1.3 \mathrm{D}_{0}$, suggesting that the sensitivity of the 
contractile machinery for $\left[\mathrm{Ca}^{2+}\right]_{\mathrm{i}}$ was increased by distension during noradrenaline activation. It has been reported previously that the effect of $\left[\mathrm{Ca}^{2+}\right]_{i}$ is higher during noradrenaline activation than during activation with potassium (Himpens et al., 1990; Nishimura et al., 1990; Jensen et al., 1992), and it is possible that the mechanism responsible for this effect is sensitive to distension. Thus in the presence of noradrenaline, distension may increase the calcium sensitivity of the contractile machinery resulting in greater contractile responses for a similar $\left[\mathrm{Ca}^{2+}\right]_{i}$ (Fig. 8.3). Previously we have shown that a phorbol ester, that activates protein kinase $C$, resulted in a similar shift in sensitivity to the contractile effect of potassium, but not noradrenaline, as is observed with distension of a vessel (Boonen and De Mey, 1991). This was suggested to be an effect of protein kinase C on calcium influx pathways and on the sensitivity of the contractile apparatus for calcium. Because noradrenaline induces formation of diacylglycerol which activates protein kinase C (Nishizuka, 1986), and because this enzyme has been shown to increase the sensitivity of the myosin light chain kinase (MLCK) for calcium, protein kinase $C$ could be involved in the observed hypersensitivity following distension. Effects of physical forces and cellular deformation on the activity of protein kinase $C$ have been observed in striated muscle (Watson, 1991) but remain to be firmly established for arterial smooth muscle. Taken together, hypersensitivity of contractile responses to noradrenaline with high distension of an isolated vessel segment could be the result of changes in membrane potential but also of alterations in the calcium sensitivity of the contractile apparatus. The different behaviour of contractile responses to high potassium in this respect, may be due to the recently described attenuation of the calcium sensitivity of myosin light chain kinase in vascular smooth muscle by depolarisation (Gilbert et al., 1991).

In conclusion, distension of isolated small mesenteric arteries resulted in a hypersensitivity to potassium as well as noradrenaline. Hypersensitivity to the contractile effect of potassium and possibly also noradrenaline can be explained partly by a slight but significant depolarisation of the vascular smooth muscle cells with a consequent increase in $\left[\mathrm{Ca}^{2+}\right]_{\mathrm{i}}$. However, changes in sensitivity and maximal responses can not solely be explained by differences in membrane potential. Results obtained with both potassium and noradrenaline suggest that there are mechanisms beyond the cell membrane in addition to an effect of overlap between myosin and actin that are involved in distensiondependent changes in contractile responses. Sensitisation of the contractile machinery for intracellular calcium by second messengers activated by noradrenaline (Himpens et al., 1990; Nishimura et al., 1990; Jensen et al., 1992) is a possible candidate in this respect. 



\section{Loss of Hypersensitivity to Vasoconstrictors in Mesenteric Muscular and Resistance Arteries of Spontaneously Hypertensive Rats Following Tissue Culture}

Harrie C.M. Boonen, Danielle H.F. Pelzer and Jo G.R. De Mey Department of pharmacology, University of Limburg, Maastricht, the Netherlands. 


\section{SUMMARY}

Blood vessels from adult hypertensive rats show hyperreactivity and hypersensitivity to vasoconstrictor agents. It is not clear whether these occur prior to or as a consequence of the elevated blood pressure. To exclude influences of pressure or pressor substances on vascular reactivity we maintained superior mesenteric (MA) and mesenteric resistance-sized arteries ( $\mathrm{MrA}$ ) from adult spontaneously hypertensive (SHR) and age matched Wistar Kyoto rats (WKY) in tissue culture under non-distending conditions and in the absence of vasoconstrictors. The experiments were performed in ring segments of MA and MrA that had been isolated, sympathectomised and maintained in tissue culture in nutrient culture medium supplemented with serum that had been dialysed to remove low molecular weight contractile factors. Following 0, 1, 2 or 4 days of culture, MA and MrA were mounted in a myograph for recording of mechanical activity. Despite stimulation of significant DNA synthesis, tissue culture did not alter maximal contractile responses to high potassium or serotonin (5HT) in MA and MrA from either SHR or WKY, whereas it reduced maximal responses to vasopressin (AVP) in MA and to phenylephrine (PHE) in MrA and MA of both strains. Compared to WKY, MA from SHR were originally hypersensitive to 5HT and AVP and hyperreactive to AVP. MrA were hypersensitive to PHE. These interstrain differences disappeared within 1 to 2 days of culture. These data indicate that qualitative differences in contractile reactivity of MA and MrA of SHR are rapidly reversible in the absence of pressure and pressor influences. They may indicate that vascular hypersensitivity in hypertension could be a secondary adaptation.

\section{INTRODUCTION}

Hyperreactivity and hypersensitivity of vascular smooth muscle to vasoconstrictors has been observed in several cardiovascular diseases (Lais and Brody, 1978; Webb, 1984). In hypertension these are not restricted to essentially hypertensives (Akbar et al., 1989) but are also commonly observed in renal and DOCA/salt hypertension, and coarctation hypertension (Jones et al., 1988; Triggle, 1989). Generally, it is believed that hyperreactivity and hypersensitivity of the arterial tree contributes to a large extent to the elevated vascular resistance in hypertensive individuals (Laher and Triggle, 1983; Mulvany, 1983; Kishi and Inoue, 1990). These alterations can be brought about by various mechanisms. Receptor number and affinity, as well as second messenger systems may have undergone modifications (Remmal et al., 1988; Kojima et al., 1989; Michel et al., 1990). Cellular key processes in the excitation contraction coupling mechanism, such as the handling of calcium, could contribute to hyperreactivity or hypersensitivity to vasoconstrictors, (Sharma and Bhalla, 1988; Uehara et al., 1988; Boonen and De Mey, 1990). Besides these alterations at the basis of the signal transduction path, hyperreactivity may result from structural vascular changes (Folkow, 1978), impairment of endothelial modulatory mechanisms (Vanhoutte, 1989; Lüscher, 1990) or from hyperactivity of neuro-endocrine mechanisms (Lee and Smeda, 1985; Sahlgren et al., 1986; Head, 1989; Resink et al., 1989; de Champlain, 1990). Furthermore, vascular hyperreactivity and 
hypersensitivity to vasoconstrictors may develop as a consequence of elevated blood pressure.

We investigated whether differences in vascular reactivity and sensitivity of arterial smooth muscle between spontaneously hypertensive rats and normotensive controls persist in the absence of pressure and pressor influences. To isolate vascular smooth muscle from the modulatory influences it experiences in vivo we made use of a tissue culture technique in which vessel integrity is preserved to allow evaluation of contractile force development (De Mey et al., 1989; Boonen et al., 1991). This approach allows controlled in vitro conditions while the vascular microenvironment remains intact (De Mey et al., 1989; Boonen et al., 1991). The function, reactivity and sensitivity to vasoactive drugs of muscular and resistance sized mesenteric arteries that had been isolated from spontaneously hypertensive and normotensive rats were followed during a four day period of tissue culture.

\section{MATERIAL AND METHODS}

Experiments were performed in superior mesenteric arteries (MA) and fourth order resistance sized side branches $(\mathrm{MrA})$ of this artery that had been dissected from the mesentery of male 20 week old spontaneously hypertensive rats (SHR) and age matched Wistar Kyoto rats (WKY)(local inbred strains, Maastricht, The Netherlands). To exclude influences of sympathetic nerve endings in the vessel wall on vascular reactivity before and during tissue culture we chose to chemically destroy these from the start. Segments that had been isolated and collected in Hank's balanced salt solution (HBSS) were therefore incubated for 10 minutes at $37^{\circ} \mathrm{C}$ in bicarbonate-free Krebs-Ringer solution (pH 4.0) that contained $300 \mu \mathrm{g} / \mathrm{ml}$ 6-hydroxydopamine (Aprigliano and Hermsmeyer, 1977). Following sympathectomy, preparations were rinsed twice for 10 minutes in $5 \mathrm{ml}$ HBSS. Vessel segments were cut into four separate parts (length approximately $2 \mathrm{~mm}$ ) of which three were prepared for tissue culture and one for direct measurement of contractile reactivity.

\section{Tissue culture of arterial ring segments}

Ring segments of MA and MrA were suspended in tissue culture as was described previously (De Mey et al., 1989; Boonen et al., 1991). Briefly, MA and MrA were supported on stainless steel wires which had diameters $(0.25$ and 0.04 $\mathrm{mm}$, respectively) that were sufficiently smaller than the slack dimension of the arteries in order to maintain the segments without physical distension. Segments were individually suspended in $25 \mathrm{~mm}$ culture wells filled with $2.5 \mathrm{ml}$ Dulbecco's Eagle's modified culture medium (DMEM) containing $100 \mu \mathrm{g} / \mathrm{ml}$ streptomycin, $100 \mathrm{IU} / \mathrm{ml}$ penicillin, $0.2 \mathrm{mM}$ glutamine and $20 \%$ fetal calf serum. The latter had been dialysed ( cut off $<6 \mathrm{kDa}$ ) to remove low molecular weight contractile components (De Mey et al., 1989). As judged from bioassay (De Mey et al., 1989), the culture medium that was used induced only a modest in- 
crease in tone in various rat arteries. Culture dishes were incubated at $37^{\circ} \mathrm{C}$ in an atmosphere of $95 \%$ air and $5 \% \mathrm{CO}_{2}$ at $97 \%$ humidity for up to 4 days. Arterial segments were transferred to culture dishes containing fresh medium after 1,2 and 3 days of culture. To have an indication of the extent of DNA synthesis, the medium contained $1 \mu \mathrm{M}$ of the thymidine analogue 5-bromo-2'-deoxyuridine (BrdU) (Gratzner, 1982; De Mey et al., 1989; Boonen et al., 1991).

\section{Contractile reactivity}

Freshly isolated arteries or arteries that had been maintained in culture for 1, 2 or 4 days were mounted on two stainless steel wires (diameter $40 \mu \mathrm{m}$ ) in an organ chamber between a displacement device and a force transducer (Kistler Morse DSC 6, Seattle, USA). The organ bath was maintained at $37^{\circ} \mathrm{C}$ and aerated with $95 \% \mathrm{O}_{2}$ and $5 \% \mathrm{CO}_{2}$ (Mulvany and Halpern, 1977). All preparations were set at their individual optimal lumen diameter for mechanical performance by stepwise stretching and intermittent activation with $125 \mathrm{mM}$ potassium solution (K-KRB) (De Mey and Brutsaert, 1984). The diameter-resting wall tension curve was used to determine a stiffness factor, i.e. the slope of the relationship between diameter and the natural logarithm of the resting wall tension (Freslon and Guidicelli, 1985). The Krebs solution had the following composition (in $\mathrm{mM}$ ): $\mathrm{NaCl} 118.5 ; \mathrm{KCl} 4.7 ; \mathrm{MgSO}_{4}$ 1.2; $\mathrm{KH}_{2} \mathrm{PO}_{4}$ 1.2; $\mathrm{NaHCO}_{3} 25.0 ; \mathrm{CaCl}_{2} 2.5 ;$ glucose 11.1. K-KRB had a similar constitution as $\mathrm{KRB}$ except that all $\mathrm{NaCl}$ was replaced by an equimolar amount of $\mathrm{KCl}$. Contractile responses were expressed as increase in wall tension which equals force development divided by twice the vessel segment length. Contractile reactivity and sensitivity of freshly isolated and cultured mesenteric arteries to different vasoactive substances were evaluated. For this purpose, cumulative concentration response curves were constructed for $0.1 \mathrm{nM}$ - $30 \mathrm{nM}$ arginine vasopressin (AVP), $1 \mathrm{nM}-30 \mu \mathrm{M}$ serotonin $(5 \mathrm{HT})$ and $1 \mathrm{nM}-30 \mu \mathrm{M}$ phenylephrine (PHE). Also relaxing responses of the vessels were assessed by construction of concentration response curves for 10 $\mathrm{nM}-1 \mu \mathrm{M}$ isoproterenol (ISO) and $10 \mathrm{nM}-1 \mu \mathrm{M}$ acetylcholine (ACH) on top of precontractions induced by $30 \mathrm{mM} \mathrm{K-KRB}$.

\section{Kinetics of DNA synthesis during tissue culture}

To evaluate growth kinetics in the vascular wall during tissue culture, vessels were fixed in periodate-lysine-paraformaldehyde for 30 minutes $\left(37^{\circ} \mathrm{C}\right)$ at optimal lumen diameter following the reactivity measurements. Preparations were embedded in paraffin. MrA and MA were either longitudinally sectioned or cross sectioned ( $4 \mu \mathrm{m}$ thick). Sections were stained with haematoxylin and eosin or immunohistochemically with an indirect enzyme-labelled antibody (Eurodiagnostics, Apeldoorn, The Netherlands) technique and a peroxidaseconjugated second step to visualise nuclei that had incorporated BrdU (Gratzner, 1982). DNA synthesis was quantified as the percentage of nuclei that stained for peroxidase in the medial or in the adventitial layer (De Mey et al., 1989; Boonen et al., 1991). 


\section{Drugs and solutions}

For tissue culture the following agents were used: Dulbecco's Eagles modified culture medium (GIBCO, Paisley, UK), penicillin (Gist-Brocades, The Netherlands), streptomycin (Pharmachemie, Haarlem, The Netherlands), 5bromo-2'-deoxyuridine (Sigma Chemicals, St. Louis, MO, USA) and 20\% fetal whole blood serum (Seralab, Sussex, UK). For the reactivity studies stock solutions of the following drugs were always prepared at the day of use in bidistilled water: arginine vasopressin (Sandoz, Basel, Switzerland), l-phenylephrine hydrochloride and l-isoproterenol hydrochloride (Sigma) and acetylcholine hydrochloride and serotonin creatinine sulphate (Janssen Chimica, Beerse, Belgium).

\section{Statistical analysis}

Maximum responses and sensitivities for the vasoconstrictors and dilators were calculated by a least square sigmoidal curve fit of the data (Graphpad Inplot, Software, San Diego, CA, USA). Effects of tissue culture on vascular reactivity and sensitivity were evaluated by analysis of variance followed by a modified t-test according to Bonferroni (Wallenstein et al., 1980). Differences between WKY and SHR were assessed using Student's unpaired t-test. $\mathrm{P}<0.05$ was considered to denote statistical significance of differences. A software package for personal computer was used for statistical analysis (Crunch Interactive Statistical Package (CRUNCH), Software Corp., San Francisco, CA, USA).

\section{RESULTS}

Mechanical properties of freshly isolated arteries are depicted in Table 9.1. The optimal lumen diameter, stiffness and maximal force development in response to $125 \mathrm{mM} \mathrm{K-KRB}$ were similar in MrA from WKY and SHR. On the other hand, the optimal lumen diameter of MA was significantly smaller in SHR than WKY (Table 9.1). The maximal force development in response to 125 $\mathrm{mM} \mathrm{K-KRB}$ in MrA and MA tended to be increased in SHR, but not significantly (Table 9.1). Stiffness, however was equal in MA of both SHR and WKY (Table 9.1).

In general, maximal contractile responses to vasoconstrictors tended to be larger in SHR than in WKY in both MrA and MA (Fig. 9.1). MA of SHR were hyperreactive to vasopressin (Fig. 9.1). Freshly isolated MA of SHR were hypersensitive to vasopressin and serotonin and MrA of SHR were more sensitive to phenylephrine than MrA from WKY rats (Fig. 9.1). Freshly isolated MA and MrA which were precontracted with $30 \mathrm{mM}$ K-KRB relaxed in response to acetylcholine and isoproterenol. Sensitivities for the $\beta$-adrenergic agonist isoproterenol were equal in the comparable vessels of SHR and WKY (Fig. 9.3). 

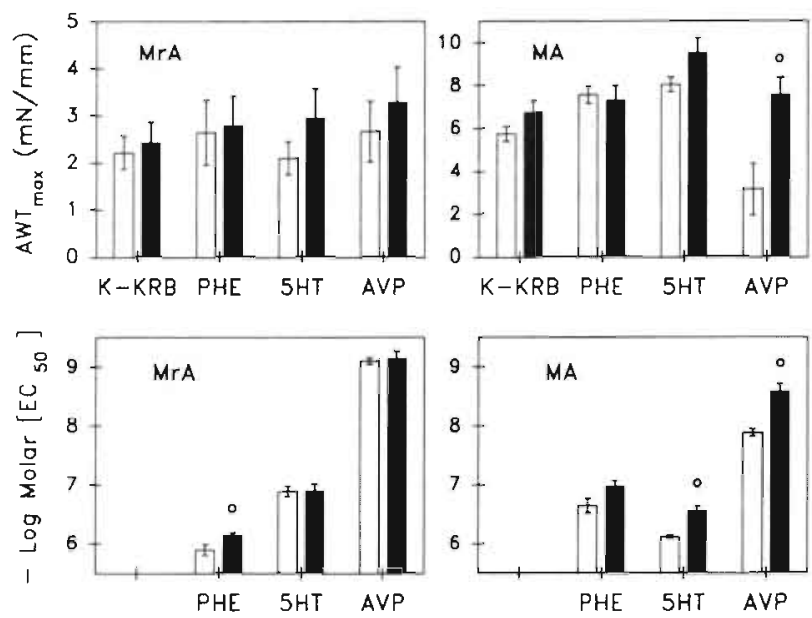

Figure 9.1. Maximal active wall tension $\left(A W T_{\max }\right.$ ) and sensitivities (- Log Molar $\left[E C_{50}\right]$ ) to different vasoconstrictors in freshly isolated sympathectomised mesenteric resistance (MrA) and superior mesenteric (MA) arteries from spontaneously hypertensive (filled bars) and Wistar Kyoto rats (open bars). Shown are means \pm SEM $(n=6)$. o indicates significance of difference between SHR and WKY (unpaired t-test, $P<0.05$ ).

\section{Vascular reactivity of large and small mesenteric arteries following tissue culture}

Effects of tissue culture in the absence of pressure and pressor substances on mechanical properties of MrA are shown in Table 9.1. Up to four days of tissue culture of MrA, derived from SHR and WKY, did not affect their responsiveness to $125 \mathrm{mM} \mathrm{K-KRB} \mathrm{(Fig.} \mathrm{9.2).} \mathrm{If} \mathrm{anything,} \mathrm{maximal} \mathrm{contractile}$ responses to $125 \mathrm{mM} \mathrm{K-KRB}$ in MrA of SHR were significantly increased compared to vessels from WKY following 24 hours of culture (Fig. 9.2). Tissue culture of MrA significantly affected contractile reactivity and sensitivities to some vasoconstrictors. Like contractile responses to $125 \mathrm{mM} \mathrm{K}-\mathrm{KRB}$ those to serotonin were hardly affected by the period in culture (Fig 9.3).

Responses to the $\alpha_{1}$-adrenoceptor agonist phenylephrine, on the other hand, were reduced following 2 and 4 days of tissue culture in MrA of both SHR and WKY (Fig. 9.3). The difference in sensitivity for phenylephrine that existed originally between SHR and WKY (Fig. 9.1) in MrA disappeared after 2 days of tissue culture (Fig 9.4). Maximal contractile responses to vasopressin in MrA did not change as a result of tissue culture (Fig. 9.3) although the sensitivity to vasopressin was reduced in MrA of WKY but not SHR (Fig. 9.4). Relaxing responses to isoproterenol in MrA were not affected by tissue culture (Fig. 9.3).

Endothelium-dependent relaxing responses induced by acetylcholine, however, were completely abolished after 24 hours of tissue culture in both MrA and MA (data not shown). 
Table 9.1. Effects of tissue culture on mechanical properties of isolated mesenteric arteries.

\begin{tabular}{|c|c|c|c|}
\hline & $\mathrm{D}_{0}(\mu \mathrm{m})$ & $\begin{array}{l}\text { Stiffness } \\
\left(\mathrm{mm}^{-1}\right)\end{array}$ & $\begin{array}{c}\mathrm{AWT}_{\max }(\mathrm{mN} \\
/ \mathrm{mm})\end{array}$ \\
\hline MrA & WKY & & \\
\hline freshly isolated & $181 \pm 15$ & $29.1 \pm 2.0$ & $2.22 \pm 0.35$ \\
\hline 1 day culture & $202 \pm 9$ & $30.4 \pm 3.1$ & $1.97 \pm 0.36$ \\
\hline $2 "$ & $209 \pm 12$ & $24.7 \pm 1.8$ & $2.70 \pm 0.24$ \\
\hline $4 \quad$ & $186 \pm 14$ & $31.8 \pm 3.9$ & $1.79 \pm 0.28$ \\
\hline MrA & SHR & & \\
\hline freshly isolated & $186 \pm 14$ & $31.6 \pm 2.5$ & $2.43 \pm 0.42$ \\
\hline 1 day culture & $212 \pm 20$ & $25.6 \pm 4.8$ & $3.43 \pm 0.46^{b}$ \\
\hline $2 "$ & $199 \pm 10$ & $29.4 \pm 2.4$ & $2.94 \pm 0.26$ \\
\hline $4 \quad "$ & $192 \pm 27$ & $29.2 \pm 3.7$ & $2.51 \pm 0.36$ \\
\hline MA & WKY & & \\
\hline freshly isolated & $1437 \pm 29$ & $5.24 \pm 0.19$ & $5.74 \pm 0.34$ \\
\hline 1 day culture & $1321 \pm 46$ & $4.45 \pm 0.49$ & $6.12 \pm 0.44$ \\
\hline $2 \quad "$ & $1204 \pm 61^{\circ}$ & $5.41 \pm 0.63$ & $5.58 \pm 0.45$ \\
\hline $4 \quad "$ & $1153 \pm 57^{a}$ & $5.21 \pm 0.47$ & $4.12 \pm 0.43^{n}$ \\
\hline MA & SHR & & \\
\hline freshly isolated & $1204 \pm 41^{b}$ & $5.37 \pm 0.46$ & $6.75 \pm 0.53$ \\
\hline 1 day culture & $1154 \pm 67$ & $5.20 \pm 0.26$ & $5.36 \pm 0.59$ \\
\hline $2 \quad "$ & $1170 \pm 44$ & $4.93 \pm 0.41$ & $6.02 \pm 0.57$ \\
\hline $4 \quad "$ & $1102 \pm 49$ & $5.41 \pm 0.43$ & $4.15 \pm 0.50^{\mathrm{s}}$ \\
\hline
\end{tabular}

Effects of tissue culture on mechanical properties of superior mesenteric (MA) and mesenteric resistance arteries ( $\mathrm{MrA}$ ) isolated from spontaneously hypertensive (SHR) and Wistar Kyoto rats (WKY). $D_{0}$ represents the diameter at which vessels contracted maximally $\left(A W T_{\max }\right.$ ) in response to $125 \mathrm{mM} \mathrm{K-KRB}$. Stiffness of the vessels was calculated from the diameter - resting tension curve (see text). Data are means $\pm \operatorname{SEM}(n=6) .{ }^{a b}$ Indicate significance of difference as compared to freshly isolated arteries or WKY controls, respectively.

In MA from WKY rats, the optimal lumen diameter was significantly decreased after 2 and 4 days of tissue culture (Table 9.1), while in MA from SHR lumen diameter remained unaffected (Table 9.1). Unlike in MrA, in MA of both SHR and WKY, maximal contractile responses to $125 \mathrm{mM} \mathrm{K-KRB}$ endured 


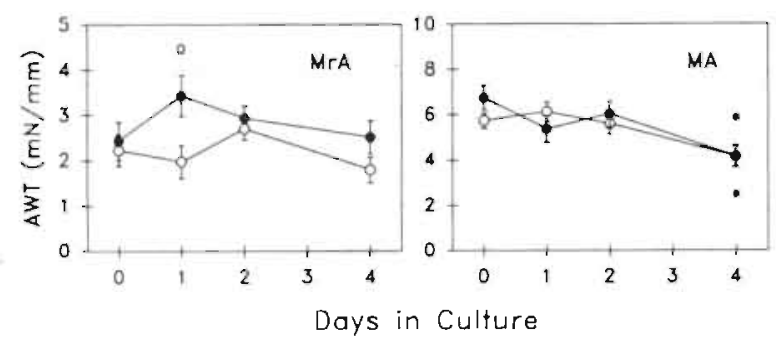

Figure 9.2. Effects of tissue culture of sympathectomised mesenteric resistance (MrA) and muscular (MA) arteries derived from spontaneously hypertensive (filled circles) and Wistar Kyoto rats (open circles) on the maximal force development (AWT) in response to stimulation with $125 \mathrm{mM}$ potassium. All data are shown as means \pm SEM $(\mathrm{n}=6)$. o indicates statistical significance of difference when compared to WKY (unpaired t-test, $P$ $<0.05)$. * denotes statistical significance of differences compared to freshly isolated arteries $(T=0)(A N O V A+t$-test (Wallenstein et al., 1980).

culture for two days but were reduced after 4 days of tissue culture (Fig. 9.2). Maximal contractile responses to serotonin were, like in MrA, unaffected by tissue culture of MA (Fig. 9.3). However, the difference in sensitivity for serotonin between MA of SHR and WKY (Fig. 9.1) was abolished after 1 day of tissue culture (Fig. 9.4).

In MA of SIRR and WKY, responses to vasopressin were differently affected. The difference in maximum response that was originally present between MA of SHR and WKY (Fig. 9.1) faded during culture (Fig. 9.3). Also the hypersensitivity of SHR for vasopressin disappeared during tissue culture (Fig. 9.4). As in $\mathrm{MrA}$, tissue culture did not affect the maximal $\beta$-adrenoceptor mediated relaxations to isoproterenol in MA of both strains (Fig. 9.3). Yet, in MA of WKY but not SHR at the fourth day of tissue culture, the sensitivity to isoproterenol was increased (Fig. 9.4).

\section{Effects of tissue culture on DNA synthesis}

During the four days of tissue culture vessels remained in an environment that contained a large concentration of classical growth factors. To asses whether this affected the synthesis of DNA or the total number of nuclei per cross section, vessels were continuously exposed to BrdU. Incorporation of the thymidine analogue was visualised and quantified. Figure 9.5 shows the effects of tissue culture on the cumulative incorporation of BrdU in the medial and adventitial layer of MrA and MA of WKY rats. Although DNA synthesis in adventitial fibroblasts seemed to be larger in MA than in MrA this difference 


\section{LOSS OF HYPERSENSITIVITY FOLLOWING CULTURE}

A

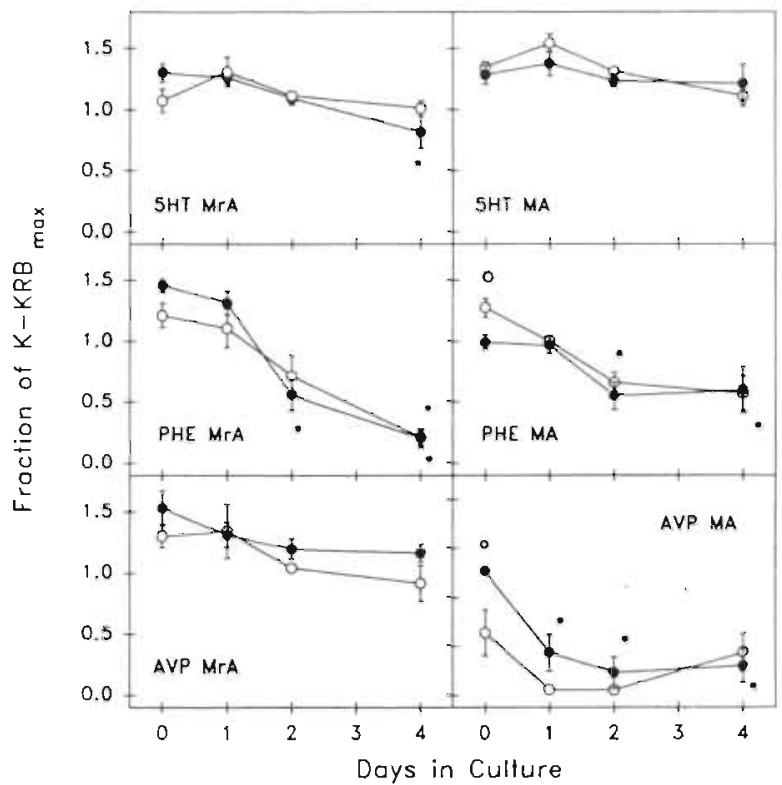

Figure 9.3. A) Effects of tissue culture on contractile reactivity to serotonin (5HT), phenylephrine (PHE) or arginine vasopressin (AVP) of isolated mesenteric resistance arteries (MrA) and superior mesenteric arteries (MA) from spontaneously hypertensive (filled circles) and Wistar Kyoto (open circles) rats. Data are shown as a fraction of the maximal response that was induced by exposure of the vessels to $125 \mathrm{mM} \mathrm{K-KRB}$. Mean \pm SEM ( $\mathrm{n}$ $=6$ ). 0 and * indicate statistical significance of difference when compared to WKY (unpaired t-test, $P<0.05$ ) or freshly isolated arteries (ANOVA $+t$-test (Wallenstein el al, 1980), respectively. B) Effects of tissue culture on relaxing responses to the $\beta$-adrenoceptor agonist isoproterenol in MA and MrA of SHR and WKY. Graphs have a similar layout as in figure $9.3_{A}$, except that data are displayed as maximal relaxation as a fraction of the response to the initial precontraction induced by $30 \mathrm{mM} \mathrm{K}-\mathrm{KRB}$.

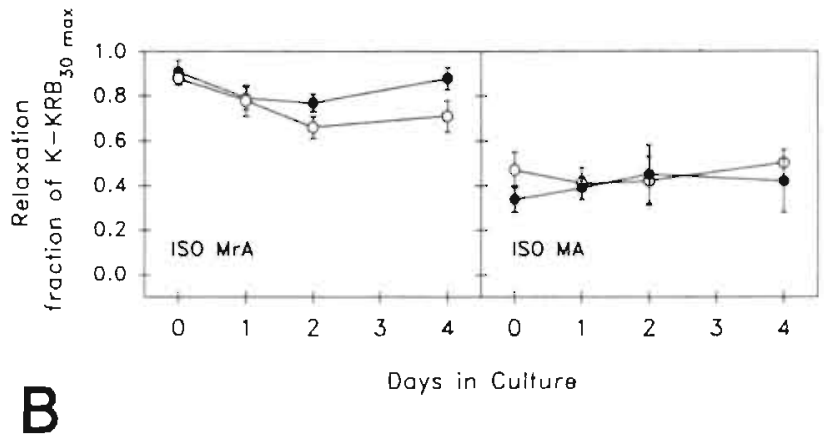


A

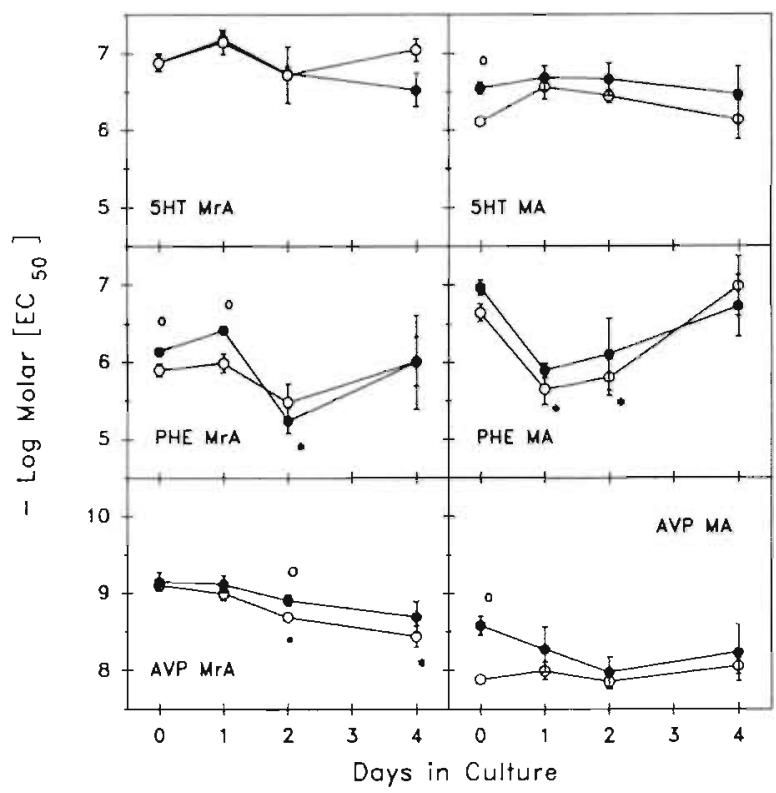

Figure 9.4. Sensitivities to A) the vasoconstrictors serotonin (5HT), phenylepturine (PHE) and arginine vasopressin (AVP) and B) vasodilator isoproterenol (ISO) in mesenteric resistance arteries $(\mathrm{MrA})$ and superior mesenteric arteries (MA) that were isolated from spontaneously hypertensive (filled circles) and Wistar Kyoto rats (open circles) and subjected to tissue culture in the absence of pressure and pressor substances. Shown are means \pm SEM of the negative logarithm of the concentrations of agonists needed to induce half of the maximal effect $\left(\mathrm{EC}_{50}\right)$. o and ${ }^{*}$ indicate statistical significance of difference from WKY or freshly isolated vessels, respectively.

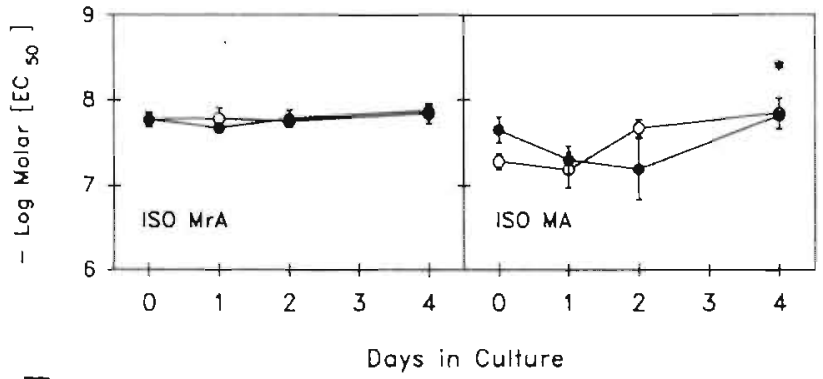

B 


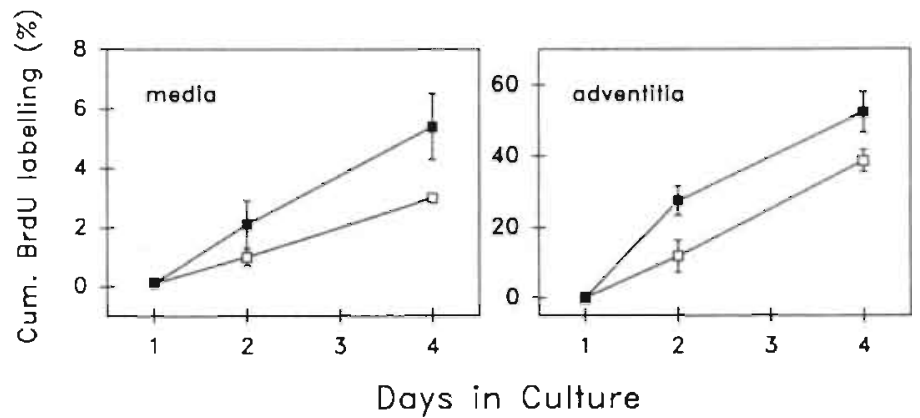

Figure 9.5. Cumulative incorporation of BrdU in medial (left hand side) and adventitial (right hand side) nuclei during culture of mesenteric resistance arteries (open squares) and superior mesenteric arteries (filled squares) of Wistar Kyoto rats. Sympathectomised arterial segments were continuously exposed to $1 \mu \mathrm{M}$ BrdU during the culture period. The percentage of labelled nuclei is shown as means \pm SEM ( $n=5-6$ segments).

Table 9.2. Effects of tissue culture on number of nuclei in medial and adventitial layers of small and large mesenteric arteries.

\begin{tabular}{lcc|cc}
$\begin{array}{l}\text { nuclear profiles / } \\
\text { section }\end{array}$ & \multicolumn{2}{c}{ MrA } & \multicolumn{2}{c}{ MA } \\
\hline days in culture & media & adventitia & media & adventitia \\
\hline 0 & $291 \pm 36$ & $49 \pm 9$ & $554 \pm 30$ & $92 \pm 14$ \\
1 & $276 \pm 19$ & $61 \pm 7$ & $622 \pm 28$ & $108 \pm 8$ \\
2 & $270 \pm 39$ & $59 \pm 8$ & $645 \pm 22$ & $119 \pm 5$ \\
4 & $239 \pm 28$ & $74 \pm 5$ & $627 \pm 62$ & $149 \pm 15^{a}$ \\
\hline
\end{tabular}

Effects of tissue culture of mesenteric arteries on the number of nuclear profiles per cross (MA) or longitudinal (MrA) section. Plasia was estimated by counting the number of nuclei that stained with haematoxylin plus the number of those that stained positive for BrdU. Data are means \pm SEM $(n=5-6)$. "indicates statistical significance of difference as compared to freshly isolated arteries (ANOVA + t-test (Wallenstein, 1980), $P<0.05$ ).

did not reach statistical significance (Fig. 9.5). DNA synthesis in the media was, compared to that in adventitial nuclei, significantly lower (Fig 9.5). The slope of the cumulative BrdU labelling versus time was significantly smaller in the media of $\mathrm{MrA}$ (slope equals $0.0075 \pm 0.0025, \mathrm{n}=6$ ) than in the medial layer of MA (slope $0.014 \pm 0.0023, n=6$ )(Fig 9.5). Despite significant synthesis of DNA, the total number of nuclear profiles in the adventitia and media of both MrA and MA was not altered after up to 4 days of culture (Table 9.2). 


\section{DISCUSSION}

Tissue culture of mesenteric muscular and resistance-sized arteries of spontaneously hypertensive rats in the absence of pressure and pressor influences resulted in normalisation of qualitative differences in vascular reactivity to vasoconstrictors compared to vessels of normotensive controls. This may indicate that vascular hypersensitivity develops as a consequence of hypertension.

Observations are not in agreement regarding the question whether changes in vascular function are primary or secondary to the development of hypertension. Differences in arterial sensitivity to vasoconstrictors could be inherent to spontaneously hypertensive rats and may contribute to the development of the hypertensive state (Smeda et al., 1988; Mulvany, 1990). However, it has also been observed that changes in sensitivity to vasoconstrictors develop as a consequence of altered blood pressure (McMahon and Paul, 1985; Triggle, 1989). To draw conclusions concerning the causal or adaptive nature of alterations in vascular sensitivity from experiments in vivo is complicated by the presence of unknown intervening factors. For example, haemodynamics and neurohumoral "status" may be primary, or secondary to the change in blood pressure and some factors may even be epiphenomena, unrelated to blood pressure (Korner and Swales, 1991). Cell cultures of isolated vascular smooth muscle cells have therefore been used to isolate the cells from the modulatory influences they undergo in vivo (Chamley-Campbell et al., 1979). Cell cultures of smooth muscle cells isolated from large arteries of hypertensive rats have demonstrated significant increases in calcium influx induced by vasoconstrictors (Bukoski, 1990) and alterations in the regulation of intracellular $\mathrm{pH}$ (Alexander et al., 1990). These alterations, that persist following many culture passages suggest that some processes that may be involved in hypersensitivity to agonists are present in the absence of elevated pressure. However, separation of smooth muscle cells from their natural microenvironment may alter their characteristics. Depending on seeding density, smooth muscle cells transform in culture from a contractile into a synthetic phenotype (Chamley-Campbell et al., 1979; Schwartz et al., 1986). This implies that functional characteristics of subcultured smooth muscle cells may not be extrapolated to the entire vessel wall (Stadler et al., 1989). Furthermore, procedures used for obtaining single smooth muscle cells may select a subset of growthprone smooth muscle cells with properties that differ from those of smooth muscle cells in a terminal state of differentiation (Owens, 1989; De Mey et al., 1991).

In an attempt to combine the advantages of in vivo experiments and culture of isolated vascular smooth muscle cells, we evaluated reactivity to vasoconstrictor agents in ring segments of small and large mesenteric arteries following tissue culture. Vessels of spontaneously hypertensive rats and normotensive controls were kept in tissue culture under similar circumstances for up to four days in the absence of pressure and of neurohumoral vasopressor substances. In analogy with previous observations (De Mey et al., 1989; Boonen et al., 1991), tissue culture of arterial ring segments induced significant synthesis of DNA in part of the medial smooth muscle cells but did not modify the number 
of nuclear profiles per cross section (Table 9.2 and Fig. 9.5). Despite this, contractile reactivity to high potassium solution was unaffected in MrA and MA for at least 2 days (Table 9.1 and Fig. 9.1), suggesting that either cells that synthesise DNA do not loose their ability to contract, as is often the case in culture of isolated cells (Chamley-Campbell et al., 1979), or that different subsets of smooth muscle cells are present in the media which are predisposed to either proliferate or contract (Owens, 1989). In this respect, comparison of superior mesenteric arteries with mesenteric resistance arteries shows that in the latter, the slope of the cumulative increase in relative DNA synthesis in the media, but not in the adventitia, is significantly less. This could be in agreement with the above mentioned hypothesis of smooth muscle cell heterogeneity in the vascular wall. The media of MrA could either consist of relatively less growth-prone smooth muscle cells or more cells that are committed to contraction (De Mey et al., 1991). Furthermore, in MA contractile reactivity was slightly decreased after 4 days of culture whereas in MrA this is not the case (Fig. 9.2). Whether this is related to the difference in relative incorporation of BrdU that is seen between MA and MrA (Fig. 9.5), remains to be established. Nevertheless, viability. defined as the capacity to contract in response to direct stimulation was largely maintained throughout the experimental period.

Originally, superior mesenteric arteries of the spontaneously hypertensive rat showed hypersensitivity and hyperreactivity to vasopressin and hypersensitivity to serotonin compared to those of Wistar Kyoto rats (fig 9.1). Resistance sized side branches of this artery, on the other hand, were hypersensitive to the $\alpha_{1}$-adrenoceptor agonist phenylephrine (Fig, 9.1). Tissue culture had profound effects on contractile responses to agonists. Responses to serotonin in both MA and MrA were preserved whereas those to phenylephrine in MA and $\mathrm{MrA}$ and vasopressin in MA were rapidly lost following 1 to 2 days of culture (Fig. 9.3). Similar findings were previously obtained during tissue culture of rat renal arteries (De Mey et al., 1989). One would rather expect $\alpha$-adrenergic responsiveness to increase as a result of sympathectomy of the arteries at the start of the culture period (Aprigliano and Hermsmeyer, 1977). Interestingly, previous studies have shown that relaxing responses to the $\beta$-adrenoceptor agonist isoproterenol in renal arteries became manifest only following tissue culture (De Mey et al., 1989), while in mesenteric arteries, the $\beta$-adrenergic responsiveness was unaffected by tissue culture (Fig 9.3 and Fig 9.4). It is unclear whether loss of responsiveness to the $\alpha_{1}$-adrenoceptor agonist is related to the increase in DNA synthesis that is seen in both arteries, but these results stress the possible heterogeneity in smooth muscle characteristics not only between branching order of arteries but also between vascular beds, as was suggested previously (De Mey et al., 1991). It is unlikely that the loss of reactivity to phenylephrine and vasopressin in MA of SHR compared to WKY can be attributed to changes in vascular structure in MA of WKY following tissue culture (Table 9.1) because structural alterations would non-selectively affect reactivity to vasoconstrictors (Folkow, 1978). As the number of nuclear profiles per cross section is unaltered (Table 9. 2) the reduced lumen diameter in MA of WKY may, however, indicate that these vessels undergo some rearrangement of passive components in their 
medial structure which may be related to the absence of flow (Langille et al., 1989).

Besides affecting reactivity in mesenteric arteries, tissue culture abolished differences in sensitivity to vasopressin and serotonin in MA and to phenylephrine in MrA of spontaneously hypertensive rats (Fig 9.4). Whether this alteration is due to loss or change of receptors, receptor-effector coupling or changes on other levels of the pharmaco-mechanical coupling pathway remains to be established. Nonetheless, these data suggest that under similar environmental circumstances i.e. without transmural pressure and in the absence of external contractile stimuli, the differences in reactivity and sensitivity to vasoactive agonists between mesenteric arteries of spontaneously hypertensive and Wistar Kyoto rats disappear.

Regardless the precise nature of the altered sensitivity to serotonin and vasopressin in $\mathrm{MA}$ and phenylephrine in $\mathrm{MrA}$, these results therefore indicate that reactivity and sensitivity of vascular smooth muscle cells to vasopressor substances may be susceptible to modification by factors outside the smooth muscle cell. The "pharmacological phenotype" of smooth muscle cells in the medial wall may be controlled by factors such as pressure, or substances that exert pressor effects. From these we conclude that vascular hypersensitivity in spontaneously hypertensive rats may be a secondary adaptation to elevated blood pressure. 


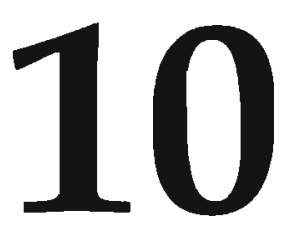

\section{Resistance Artery Changes Following} Vasoconstrictor Infusion in Young Rats. Comparison with those in Young Spontaneously Hypertensive Rats

Harrie C.M. Boonen, Mat J.A.P. Daemen a, Pedro H.A. Eerdmans, Gregorio E. Fazzi, Ellen M. van Kleef , Paul M.H. Schiffers and Jo G.R De Mey 


\section{SUMMARY}

To evaluate whether chronic $\alpha_{1}$-adrenergic stimulation, angiotensin II or elevated blood pressure alter resistance arterial reactivity and structure, we recorded structural parameters and wall tension in mesenteric small arteries ( $\mathrm{MrA}$ ) isolated from 6 weeks old normotensive Wistar Kyoto rats that had been infused for 4 days with either saline (WKY), $2 \mathrm{mg} / \mathrm{kg} /$ day phenylephrine $(W K Y+P H E)$ or $0.3 \mathrm{mg} / \mathrm{kg} /$ day angiotensin Il (WKY+AII) or from saline-infused spontaneously hypertensive rats (SHR). During the experimental period, systolic blood pressure (SBP) increased in SHR but not in WKY. In WKY + PHE and WKY +AII, SBP increased and heart rate was reduced. Cardiac weight did not differ between SHR and WKY, but was increased in WKY+PHE and WKY +AII. Stiffness and optimal lumen diameter of MrA did not differ between WKY and SHR and were not altered in WKY+PHE or WKY +AII. Maximal contractile responses of sympathectomised MrA to high potassium, calcium, angiotensin II, noradrenaline, phenylephrine and serotonin, tended to be larger in SHR and WKY+AII than in WKY. Sensitivities for the vasoconstrictors and calcium were not altered in vessels of WKY+AII and did not differ between vessels of SHR and WKY. In those of WKY+PHE, maximal responses to all constrictors and the sensitivity to noradrenaline and phenylephrine were reduced. Vasorelaxing responses to isoproterenol and Na-nitroprusside did not differ between SHR and WKY and were not altered in WKY+PHE or WKY+AII. Those to acetylcholine tended to be reduced in WKY+PHE and WKY+AII. Media cross sectional area and media thickness were significantly larger in WKY +AII and SHR compared to WKY but were not altered in WKY +PHE. These data indicate that in young rats, angiotensin II, but not increased vasoconstriction or $\alpha_{1}$-adrenergic stimulation leads to small artery hypertrophy and hyperreactivity. Increased trophic actions of angiotensin II are more likely to contribute to the development of hypertension in SHR than hypersensitivity to vasoconstrictors, hyposensitivity to vasodilators or altered calcium handling by vascular smooth muscle.

\section{INTRODUCTION}

In established hypertension, vascular resistance is increased as a result of arterial structural changes (Folkow, 1982; Lee, 1987; Mulvany, 1987). These contribute to vascular hyperreactivity and underlie increased risk for life threatening cardiovascular disorders. How they are brought about is still a matter of debate. In essential hypertension and in spontaneously hypertensive rats, part of the structural changes may be genetic in origin (Folkow, 1982; Gray, 1984). Additional modifications, and those in secondary forms of hypertension may evolve from increased supply of classical vasoconstrictors and growth factors and from mechanical stresses on the vascular wall (Lever, 1986; Sahlgren et al., 1986; Dostal et al., 1988). In young spontaneously hypertensive rats, changes in vascular reactivity to calcium, which could be relevant to both constrictor and growth responses, have been observed prior to significant elevation of blood pressure (Mulvany and Aalkjaer, 1990). In other animal models, such as renal hypertensive and DOCA/salt hypertensive rats, changes in vascular function and structure can 
develop in parallel to or as a result of the blood pressure rise (Laher and Triggle, 1984; Cox, 1987; Jimenez and Joshua, 1988; Triggle, 1989). In these adaptive processes there could be a role for both hypersensitivity of the vascular smooth muscle and for the endothelium (Lüscher 1990a). Moreover, hyperactivity of the sympathetic nervous system and the renin-angiotensin-aldosterone system have been suggested to participate in the development of hypertension (Lee and Smeda, 1985; Salligren et al., 1986; Head, 1989; Resink et al., 1989; de Champlain, 1440). Little is known, however, concerning the role of small arteries in this respect, although they are the prime effectors of hypertension.

In the present study we evaluated whether the structure, contractile reactivity and endothelium-dependent relaxing reactivity in small arteries was altered in young spontaneously hypertensive rats and following hypertension induced by continuous infusion of phenylephrine or angiotensin II in Wistar Kyoto rats. We used continuous infusion of phenylephrine and angiotensin II to mimic hyperactivity of systems that were proposed to participate in the genesis of essential and renal hypertension (Abboud, 1982; Head, 1989; Lüscher, 1990b; scott-Burden et al., 1992). The experiments were performed in 6 week old rats in which intra-arterial DNA synthesis is still significant (De Mey et al., 1991) and thus possibly prone to modification.

\section{MATERIAL AND METHODS}

\section{Animals and treatments}

6 Weeks old male normotensive Wistar Kyoto (WKY) and age and sex matched spontaneously hypertensive rats (SHR) were used (locally inbred colonies; University of Limburg, Maastricht, The Netherlands). Four groups of 8 rats each were defined. Three groups consisted of Wistar Kyoto rats that received either saline, phenylephrine (WKY+PHE) or angiotensin II (WKY+AII) infused continuously during four days through a subcutaneously implanted osmotic minipump (Alzet, model 2001, Alza, Palo Alto, CA, USA). One group consisted of spontaneously hypertensive rats that received saline. In preliminary experiments $(\mathrm{n}=4)$, infusions of WKY rats with $2 \mathrm{mg} / \mathrm{kg} /$ day phenylephrine or $300 \mu \mathrm{g} / \mathrm{kg} /$ day angiotensin II were observed to increase systolic blood pressure significantly to a level comparable to that seen in 6 week old SHR. The minipumps were implanted subcutaneously under ether anaesthesia as described before (Daemen et al., 1991; De Mey et al., 1991). Prior to implantation the filled pumps were kept for 2 hours at $37^{\circ} \mathrm{C}$ in saline to obtain a steady state delivery of their contents. Heart rate and systolic blood pressure were measured by tail cuff plethysmography (Narco Biosystems, Houston, TX, USA) on the following time points: $T=-6 ;-3 ; 0 ; 2$ and 4 days in which $T=0$ is the day on which the minipumps were implanted. Four days after the implantation of the minipumps the rats were sacrificed by bleeding from the aorta under ether anaesthesia. Heart and kidneys were dissected. 


\section{Vascular reactivity}

Fourth order side branches of the superior mesenteric artery were dissected from the mesentery. During dissection and experimentation the vessels were immersed in Krebs Ringer bicarbonate buffered salt solution (KRB). Vessels were chemically sympathectomised by incubation during $10 \mathrm{~min}$ at $37^{\circ} \mathrm{C}$ in a bicarbonate free Krebs's solution containing $300 \mu \mathrm{g} / \mathrm{ml}$ 6-hydroxydopamine (Aprigliano and Hermsmeyer, 1977). Vessels (lumen diameter $\approx 200 \mu \mathrm{m}$ ) were mounted as ring segments (length approximately $2 \mathrm{~mm}$ ) on two stainless steel wires $(\curvearrowleft 40 \mu \mathrm{m})$ between a displacement device and a force transducer (Kistler-Morse DSC-6, Seattle, USA) in a myograph that allowed measurement of isometric force development (Mulvany and Halpern, 1977). The vessels were stretched to an internal diameter at which they contracted maximally to stimulation with 125 $\mathrm{mM}$ potassium (De Mey and Brutsaert, 1984). A stiffness factor ( $\beta$ ) and the effective resting transmural pressure (ERTP) were derived from the diameter-tension curve (Freslon and Guidicelli, 1985).

At optimal lumen diameter, concentration response curves were constructed for angiotensin II $(0.1 \mathrm{nM}-1 \mu \mathrm{M})$, noradrenaline (10 $\mathrm{nM}-10 \mu \mathrm{M})$, phenylephrine $(10 \mathrm{nM}-10 \mu \mathrm{M})$ and serotonin $(10 \mathrm{nM}-10 \mu \mathrm{M})$. To avoid tachyphylaxis, concentration response curves for angiotensin II were constructed on top of a contractile response induced by $30 \mathrm{mM} \mathrm{K-KRB}$. Concentration response curves for noradrenaline were constructed in the presence of $1 \mu \mathrm{M}$ propranolol. To evaluate endothelium-dependent and independent relaxing responses (De Mey and Gray, 1985), concentration response curves for acetylcholine (10 $\mathrm{nM}-10 \mu \mathrm{M})$, isoproterenol $(1 \mathrm{nM}-1 \mu \mathrm{M})$ and sodium-nitroprusside (10 nM $10 \mu \mathrm{M}$ ) were constructed in vessels that had been precontracted with $30 \mathrm{mM} \mathrm{K}$ KRB.

Sensitivity of the vessels for extracellular calcium was evaluated by constructing concentration response curves for calcium in the continuous presence of either $10 \mu \mathrm{M}$ noradrenaline or $125 \mathrm{mM} \mathrm{K-KRB}$ (Boonen and De Mey, 1990). Therefore vessels were depleted of extracellular calcium by a $3 \mathrm{~min}$ incubation in $\mathrm{Ca}^{2+}$-free $\mathrm{KRB}$ that contained $0.3 \mathrm{mM}$ EGTA and intracellular calcium stores were emptied by 3 minute stimulation of the vessels in $\mathrm{Ca}^{2+}$-free $\mathrm{KRB}$ containing $10 \mu \mathrm{M}$ noradrenaline. Then calcium was added cumulatively $(1.25 \mu \mathrm{M}-10$ $\mathrm{mM}$ ) in the continuous presence of either $10 \mu \mathrm{M}$ noradrenaline or $125 \mathrm{mM} \mathrm{K}$ $\mathrm{KRB}$. The former were constructed in the continuous presence of $1 \mu \mathrm{M}$ propranolol.

\section{Drugs and solutions}

Krebs-Ringer bicarbonate buffered solution had the following composition (in $\mathrm{mM}$ ): $\mathrm{NaCl}, 118.5 ; \mathrm{KCl}, 4.7 ; \mathrm{MgSO}_{4} .7 \mathrm{H}_{2} \mathrm{O} \quad 1.2 ; \mathrm{KH}_{2} \mathrm{PO}_{4} 1.2 ; \mathrm{NaHCO}_{3}$ 25.0; $\mathrm{CaCl}_{2} 2.5$; and glucose 11.1. In high potassium solution (K-KRB) all $\mathrm{NaCl}$ was replaced by an equimolar amount of $\mathrm{KCl}$. In calcium free $\mathrm{KRB}, \mathrm{CaCl}_{2}$ was omitted from normal KRB. Where mentioned, $0.3 \mathrm{mM}$ EGTA was added to calcium free KRB. Acetylcholine, serotonin creatinine sulphate monohydrate 
and sudium-nitroprusside were obtained from Janssen Chimica (Beerse, Belgium). Rat angiotensin-II, Ethylene-glycol-bis-( $\left(\right.$-amino-ethyl-ether) $N, N^{\prime}$-tetraacetic acid (EGTA), isoproterenol sulphate, l-arterenol-bitartrate (noradrenaline), J-phenylephrine hydrochloride, 6-hydroxydopamine- $\mathrm{HCl}$ and propranolol-HCl were obtained from Sigma Chemical Co. (St.Louis, MO, USA). Stock solutions $(10 \mathrm{mM})$ were prepared daily in bidistilled water.

\section{Morphometry}

Following recording of contractile reactivity, vessels were fixed at the optimal lumen diameter for 30 minutes in $4 \%$ phosphate buffered (pH 7.4) paraformaldehyde and embedded in paraffin. Cross sections $(4 \mu \mathrm{m})$ were stained with Lawson's solution (Boom B.V., Meppel, The Netherlands) to visualise internal and external elastic laminae. Video images were generated from the cross sections using a Zeiss Axioscope (Zeiss, Germany) and a standard CCD camera (Stemmer, Germany). Using commercially available software (JAVA 1.21, Jandel Scientific, Corte Madera, CA, USA) the area enclosed by the internal elastic lamina was subtracted from the area enclosed by the media-adventitial border to obtain media cross sectional area (CSA). Lumen radius was calculated from the internal circumference (IC) assuming a circular cross section. From these parameters, mean media thickness $\left(\mathrm{M}_{\mathrm{t}}\right)$ was derived using the following formula:

$$
M_{t}=-r+\sqrt{r^{2}+\frac{C S A}{\pi}}
$$

\section{Data analysis}

The rats that were used were assigned a random numerical code at 5 weeks of age. Implantation of the minipumps, blood pressure measurements, reactivity studies and morphometry were performed by different investigators. Codes were broken after all observations had been obtained. Contractile responses were expressed as increases in wall tension (i.e. increases in force divided by twice the segment's length). $\mathrm{EC}_{\bar{\nabla}}$ values for agonists were determined by least square sigmoidal curve fitting (Graphpad Inplot 3.01, San Diego, CA, USA). Differences in vascular reactivity between rats treated with phenylephrine or angiotensin II and controls were evaluated by one-way analysis of variance followed by a t-test according to the method by Bonferroni (Wallenstein et al., 1480) using the Crunch Interactive Statistical Package (Crunch Software Corp., San Francisco, California, USA). P $<0.05$ was accepted to denote statistical significance of differences. 


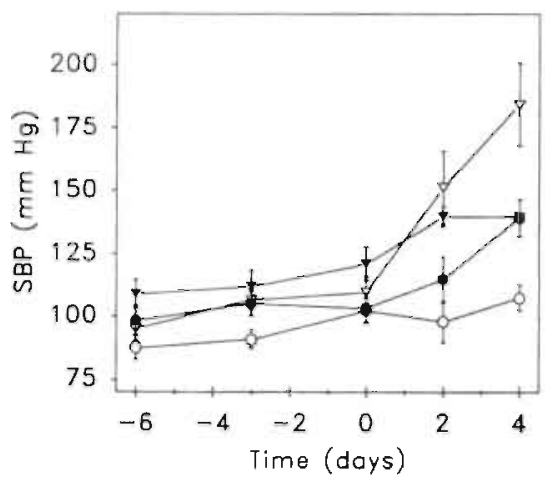

Figure 10.1. Development of systolic blood pressure measured by tail cuff plethysmography in 6 week old (at $\mathrm{T}=0$ ) WKY and SHR rats that were infused for four days (starting from $\mathrm{T}=$ 0) with saline (open circles and filled triangles, respectively) and WKY infused with phenylephrine (filled circles) or angiotensin II (open triangles). Shown are means \pm SEM $(n=8)$.

Table 10.1. Effect of vasopressor infusion on heart and kidney weight in 6 week old rats.

\begin{tabular}{lcccc} 
& WKY & WKY + PHE & WKY + AII & SHR \\
\hline $\begin{array}{l}\text { heart/body weight } \\
\text { (mg/g) }\end{array}$ & $3.96 \pm 0.11$ & $5.60 \pm 0.50^{\mathrm{a} .6}$ & $5.02 \pm 0.19^{\mathrm{a}}$ & $4.20 \pm 0.10$ \\
$\begin{array}{l}\mathrm{kidney} / \text { body weight } \\
(\mathrm{mg} / \mathrm{g})\end{array}$ & $5.29 \pm 0.07$ & $6.00 \pm 0.40$ & $5.18 \pm 0.09$ & $4.90 \pm 0.30$ \\
\hline
\end{tabular}

Relative heart and kidney weight corrected for body weight in rats that had been infused during four days with either soline (WKY and SHR), phenylephrine $(2 \mathrm{mg} / \mathrm{kg} / \mathrm{day}$ ) $(W K Y+P H E)$ or angiotensin II $(300 \mu \mathrm{g} / \mathrm{kg} /$ day $)(W K Y+$ AII $)$. Shown are mean \pm SEM $(n=6-8)$. a.t represent statistical significance of differences compared to WKY and SHR, respectively (ANOVA + t-test (Wallenstein, 1980) $P<0.05$ ).

\section{RESULTS}

Figure 10.1 illustrates systolic blood (SBP) pressures during the fifth and sixth postnatal week in WKY and SHR rats. The earliest measurements did not differ between both strains. Towards the end of the sixth week, SBP was larger in SHR than WKY (Fig. 10.1). Furthermore, regression analysis indicates that SBP rose significantly during the study period in SHR but not WKY. The slope of the regression lines (increase in SBP $(\mathrm{mmHg} /$ day)) averaged $1.27 \pm 2.23$ $(P=0.592)$ and $5.63 \pm 2.20(P=0.016)$ in $W K Y$ and SHR, respectively.

Treatment of 6 week old WKY with phenylephrine or angiotensin II caused a sustained increase in SBP (Fig. 10.1). Following 4 days of phenylephrine, SBP was comparable to that in saline-infused SHR (regression analysis showed an average slope of $8.52 \pm 2.84 ; P=0.006$ ) (Fig. 10.2). Angiotensin II increased SBP to a larger extent (slope $15.50 \pm 4.87 ; \mathrm{P}=0.004$ ) (Fig's. 10.1 and 
10.2). The bradycardia that accompanied the vasopressor treatment was, however, comparable for WKY treated with phenylephrine and those infused with angiotensin II (Fig. 10.2). Furthermore, both treatments reduced the normal gain in body weight (Fig. 10.2). This was more pronounced in WKY+PHE than in WKY+AII. Heart and kidney weight did not differ significantly between saline-infused WKY and SHR but relative cardiac mass was significantly increased in WKY after 4 days of treatment with either phenylephrine or angiotensin II (Table 10.1).

\section{Mechanical and structural properties of small arteries}

The optimal lumen diameters at which the acutely sympathectomised small mesenteric arteries contracted maximally in response to $125 \mathrm{mM} \mathrm{K-KRB}$ did not differ between the four treatment groups (Table 10.2). The resting wall tension of the vessels at this optimal lumen diameter tended to be increased in WKY + AII (Table 10.2). As a consequence also effective resting transmural pressure at the optimal lumen diameter tended to be increased in vessels of WKY treated with AII (Table 10.2). The stiffness factor was similar in all groups (Table 10.2). The maximal active wall tension the vessels developed in response to stimulation with $125 \mathrm{mM} \mathrm{K-KRB}$ was significantly smaller in vessels from WKY + PHE than in those of saline-treated WKY and SHR (Table 10.2). Vessels of SHR and WKY+AII, on the other hand, were hyperreactive to $125 \mathrm{mM}$ potassium when compared to those of WKY, but this difference was not statistically significant (Table 10.2).

Media cross sectional area and mean media thickness were significantly larger in small mesenteric arteries of WKY+AII and SHR than in those of WKY (Table 10.2). Also wall to lumen ratio was significantly larger in vessels of SHR and WKY+AII than in those of WKY (Table 10.2). No structural changes were observed in vessels of WKY +PHE (Table 10.2).

\section{Contractile reactivity of the vessels}

Vessels of SHR were hyperreactive to all vasoconstrictor stimuli (Table 10.3). This, however, did never reach statistical significance. Sensitivities to vasoconstrictors were not different between WKY and SHR. Only the sensitivity for calcium in the presence of noradrenaline tended to be increased in vessels of SHR compared to WKY, but this difference was not statistically significant (Fig. 10.5). In vessels from WKY treated with phenylephrine, contractile responses to potassium were significantly reduced compared to saline-infused WKY rats (Table 10.3). Also, the sensitivity and maximal contractile response for $\alpha_{1}$-adrenergic stimulation with phenylephrine was significantly reduced (Fig. 10.3 and Table 10.3). Furthermore, the sensitivity for noradrenaline (in the presence of propranolol) was significantly reduced in vessels of rats treated with phenylephrine (Table 10.3). In vessels of WKY treated with angiotensin-II, contractile responses to angiotensin-II, noradrenaline, potassium, phenylephrine and serotonin tended to be increased, however, not statistically significant (Fig. 
Figure 10.2. Bar graphs showing the change in body weight ( $\triangle B W$ ), systolic blood pressure ( $\triangle \mathrm{SBP}$ ) and heart rate $(\triangle H R$ ) following four days infusion of saline in 6 week old WKY (open bars) and SHR (filled bars) and phenylephrine (PHE; hatched bars) or angiotensin II (AII; cross hatched bars) in WKY rats. Data are means \pm SEM. *,\# indicate statistical significance of difference as compared to WKY or SHR, respectively. + indicates that change in $\mathrm{SBP}$ is significant (ANOVA + modified t-test (Wallenstein et al., 1980)).

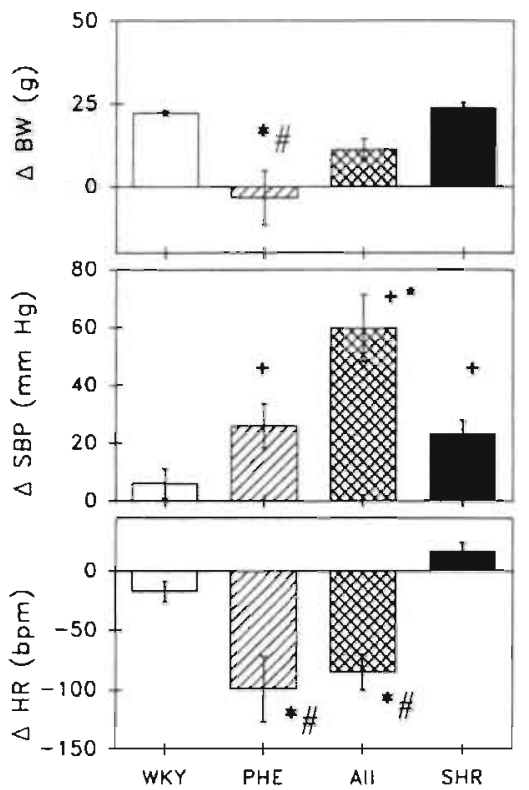

Figure 10.3. Effects of vasoconstrictors in mesenteric resistance arteries of 6 week old rats that were treated for four days with saline (WKY, open circles; SHR, filled triangles), phenylephrine (filled circles) or angiotensin II (open triangles). Concentration response curves were performed for angiotensin II (ATII)(in vessels precontracted with $30 \mathrm{mM} \mathrm{K}$ $\mathrm{KRB}$ ), noradrenaline (NA), phenylephrine (PHE) and serotonin (5HT). Symbols represent the mean \pm SEM $(n=8)$. Data are expressed as a fraction of the maximal contractile response induced by $125 \mathrm{mM}$ K-KRB.
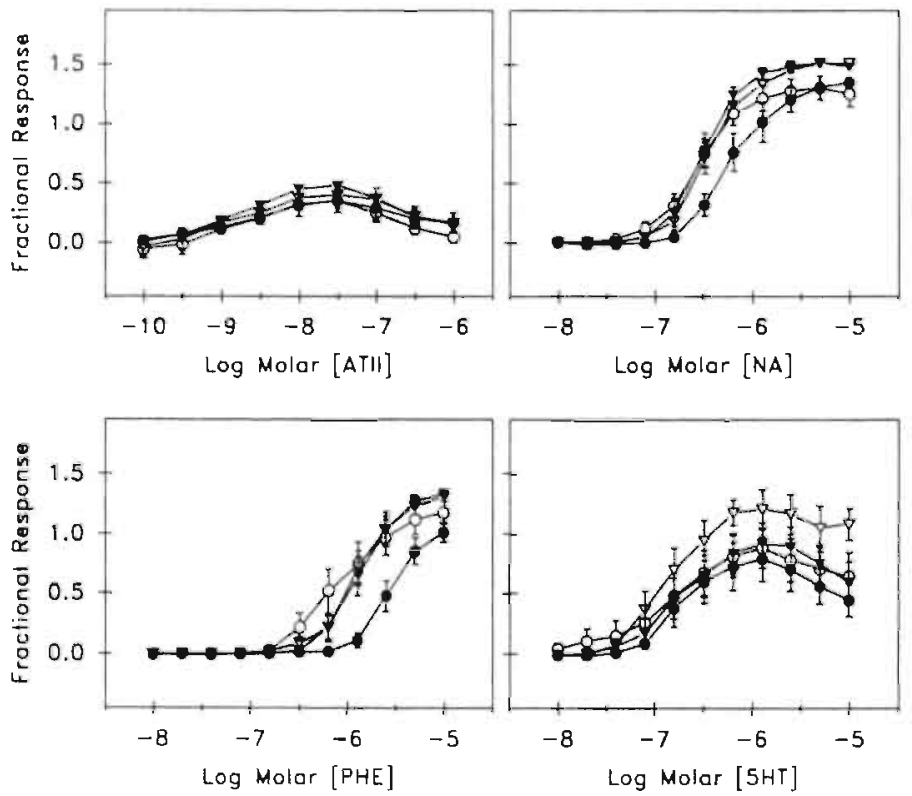
Table 10.2. Mechanical and morphological properties of isolated mesenteric resistance arteries following 4 day treatment of WKY with phenylephrine and angiotensin II.

\begin{tabular}{lcccc}
\multicolumn{1}{c}{ WKY } & WKY+PHE & WKY+All & SHR \\
\hline Mechanical properties & & & & \\
\hline OID $(\mu \mathrm{m})$ & $159 \pm 8$ & $153 \pm 12$ & $185 \pm 12$ & $180 \pm 10$ \\
RWT $(\mathrm{mN} / \mathrm{mm})$ & $0.71 \pm 0.13$ & $0.54 \pm 0.09$ & $1.13 \pm 0.19$ & $0.71 \pm 0.16$ \\
stiffness $\left(\mathrm{mm}^{-1}\right)$ & $34.5 \pm 3.4$ & $37.9 \pm 4.7$ & $28.9 \pm 2.4$ & $30.6 \pm 4.4$ \\
ERTP $\left(\mathrm{mN} / \mathrm{mm}^{2}\right)$ & $8.8 \pm 1.5$ & $7.3 \pm 1.2$ & $12.2 \pm 1.8$ & $7.5 \pm 1.6$ \\
AWT $(\mathrm{K}-\mathrm{KRB})(\mathrm{mN} / \mathrm{mm})$ & $1.81 \pm 0.10$ & $1.24 \pm 0.11^{\mathrm{a} . b}$ & $2.00 \pm 0.07$ & $2.16 \pm 0.15$ \\
\hline Morphometric parameters & & & & \\
\hline CSA $\left(\mu \mathrm{m}^{2}\right)$ & $2475 \pm 195$ & $2295 \pm 154$ & $3917 \pm 622^{\mathrm{a}}$ & $3778 \pm 336^{\star}$ \\
radius $(\mu \mathrm{m})$ & $88 \pm 2$ & $81 \pm 3$ & $90 \pm 12$ & $86 \pm 6$ \\
M $(\mu \mathrm{m})$ & $4.38 \pm 0.31$ & $4.36 \pm 0.30$ & $6.28 \pm 0.68^{\circ}$ & $6.45 \pm 0.43^{\circ}$ \\
W/L $(\times 100)$ & $5.02 \pm 0.37$ & $5.40 \pm 0.48$ & $6.67 \pm 0.45^{\star}$ & $7.31 \pm 0.59^{\circ}$ \\
\hline
\end{tabular}

WKY; Wistar Kyoto, WKY +PHE and WKY+AII; WKY rats treated during four days with pressor doses of phenylephrine or angiotensin II, respectively and SHR; spontaneously hypertensive rats. OID; optimal lumen diameter at which maximal response to $125 \mathrm{mM}$ K-KRB were obtained. RWT and AWT, resting and active wall tension, respectively. stiffness factor (see text). ERTP, effective resting transmural pressure. CSA, media cross sectional area. $M_{1}$, media thickness and $W / L$, wall to lumen ratio $\left(M_{1} / O I D\right)$. Values are means \pm SEM $(n=8)$. a.b indicate significance of difference compared to WKY or SHR, respectively (ANOVA $+\mathrm{t}$-test $\mathrm{P}<0.05$ ).

10.3 and Table 10.3). The sensitivity for extracellular calcium was not altered by treatment of WKY with phenylephrine or angiotensin II (Table 10.3 and Fig. 10.4).

Endothelium-dependent and independent relaxing properties were evaluated during contractions induced by $30 \mathrm{mM}$ potassium (Fig. 10.4). Acetylcholine-induced endothelium-dependent relaxations were comparable in vessels from saline-infused WKY and SHR but tended to be reduced in WKY rats treated with angiotensin II and phenylephrine. This difference did, however, not reach statistical significance (Table 10.3, Fig. 10.4). Relaxing responses to isoproterenol and sodium nitroprusside did not differ between the four treatment groups (Fig. 10.4). 

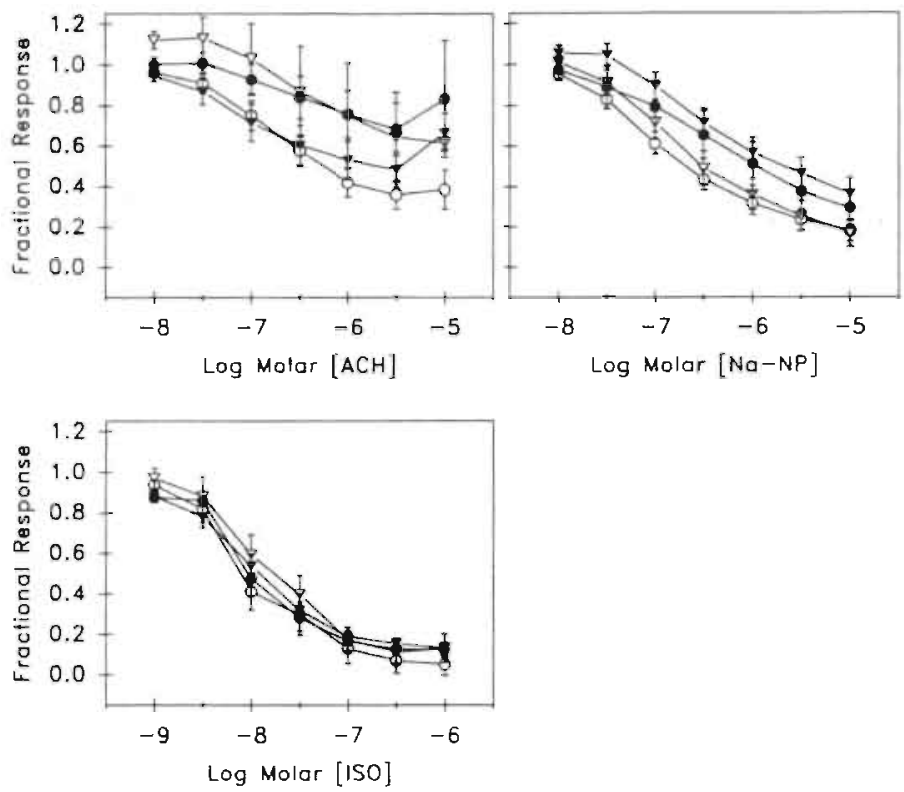

Figure 10.4. Effects of endothelium-dependent and independent vasodilator substances in mesenteric resistance arteries of 6 week old rats treated for 4 days with saline (WKKY, open circles; SHR, filled triangles), phenvlephrine (filled circles) or angiotensin II (open triangles). Displayed are means $\pm S E M$ as a fraction of the precontraction induced by 30 $\mathrm{mM} \mathrm{K-KRB.}$

Figure 10.5. Concentration response curves to extracellular calcium in mesenteric resistance arteries of 6 week old rats in the presence of $125 \mathrm{mM} \mathrm{K}-\mathrm{KRB}$ (left hand side) or 10 $\mu M$ noradrenaline (right hand side). Means \pm SEM $(n=8)$ as a fraction of the maximal response. WKY (open circles), WKY+PHE (filled circles), WKY+AII (open triangles) and SHR (filled triangles).
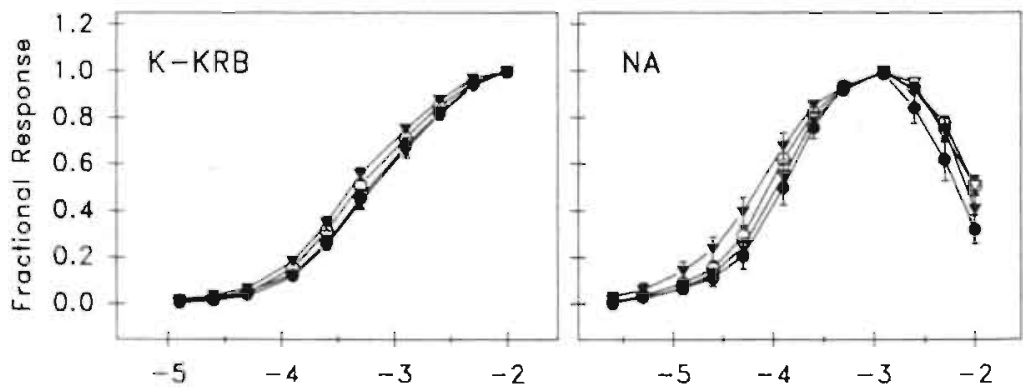

$\log \operatorname{Molar}\left[\mathrm{Ca}^{2+}\right]_{\theta}$ 


\section{SMALL ARTERY CHANGES FOLLOWLNG PRESSOR INEUSION}

Table 10.3. Sensitivities and maximal effects of vasoconstrictor and vasodilator substances in isolated mesenteric arteries

WKY
PHE

\begin{tabular}{|c|c|c|c|c|c|}
\hline \multicolumn{6}{|c|}{ vasoconstrictor substances } \\
\hline angiotensin II & $E_{\max }$ & $1.09 \pm 0.16$ & $0.65 \pm 0.18$ & $1.16 \pm 0.16$ & $1.33 \pm 0.15$ \\
\hline & $\mathrm{pD}_{2}$ & $8.75 \pm 0.13$ & $8.60 \pm 0.25$ & $8.79 \pm 0.24$ & $8.78 \pm 0.13$ \\
\hline \multirow[t]{2}{*}{ phenylephrine } & $F_{r=1}$ & $2.39 \pm 0.15$ & $1.43 \pm 0.22^{\text {at }}$ & $2.81 \pm 0.15$ & $2.88 \pm 0.29$ \\
\hline & $\mathrm{pD}_{2}$ & $6.05 \pm 0.14$ & $5.53 \pm 0.06^{a}$ & $5.90 \pm 0.10$ & $5.88 \pm 0.09$ \\
\hline \multirow[t]{2}{*}{ noradrenaline } & $E_{n t 1,1 \times}$ & $2.62 \pm 0.21$ & $1.74 \pm 0.19^{8}$ & $3.13 \pm 0.16$ & $3.25 \pm 0.31$ \\
\hline & $\mathrm{pD}_{2}$ & $6.59 \pm 0.06$ & $6.20 \pm 0.12^{\mathrm{ah}}$ & $6.49 \pm 0.06$ & $6.53 \pm 0.07$ \\
\hline \multirow[t]{2}{*}{ serotonin } & $E_{\max }$ & $1.79 \pm 0.34$ & $1.07 \pm 0.32$ & $2.64 \pm 0.25$ & $2.18 \pm 0.42$ \\
\hline & $\mathrm{pD}_{2}$ & $6.95 \pm 0.34$ & $6.68 \pm 0.12$ & $6.80 \pm 0.13$ & $6.63 \pm 0.14$ \\
\hline \multicolumn{6}{|l|}{$\left[\mathrm{Ca}^{2+}\right]_{\mathrm{e}}$} \\
\hline potassium & $\mathrm{pCa}_{2}$ & $3.25 \pm 0.10$ & $3.14 \pm 0.08$ & $3.16 \pm 0.07$ & $3.33 \pm 0.06$ \\
\hline noradremaline & $\mathrm{PCa}_{2}$ & $4.01 \pm 0.08$ & $3.90 \pm 0.08$ & $3.95 \pm 0.07$ & $4.15 \pm 0.08$ \\
\hline \multicolumn{6}{|c|}{ vasodilator substances } \\
\hline $\mathrm{K}_{30 \max }$ & $\mathrm{mN} / \mathrm{mm}$ & $1.21 \pm 0.11$ & $0.73 \pm 0.14^{h}$ & $1.11+0.12$ & $1.37 \pm 0.15$ \\
\hline \multirow[t]{2}{*}{ acetylcholine } & $E_{\max }$ & $0.65 \pm 0.07$ & $0.34 \pm 0.12^{3}$ & $0.45 \pm 0.15$ & $0.53 \pm 0.09$ \\
\hline & $\mathrm{pD}_{2}$ & $6.75 \pm 0.13$ & $6.44 \pm 0.18$ & $6.50 \pm 0.17$ & $6.88 \pm 0.22$ \\
\hline \multirow[t]{2}{*}{ isoproterenol } & $\mathrm{E}_{\operatorname{mix}}$ & $0.95 \pm 0.06$ & $0.89 \pm 0.03$ & $0.89 \pm 0.05$ & $0.88 \pm 0.03$ \\
\hline & $\mathrm{pD}_{2}$ & $7.93 \pm 0.14$ & $8.09 \pm 0.13$ & $8.04 \pm 0.27$ & $8.12 \pm 0.10$ \\
\hline \multirow[t]{2}{*}{$\mathrm{Na}$-nitroprusside } & $E_{\operatorname{minx}}$ & $0.84 \pm 0.05$ & $0.69 \pm 0.08$ & $0.85 \pm 0.05$ & $0.64 \pm 0.07$ \\
\hline & $\mathrm{pD}_{2}$ & $7.01 \pm 0.13$ & $6.43 \pm 0.28$ & $6.65 \pm 0.17$ & $6.38 \pm 0.19$ \\
\hline
\end{tabular}

Maximal effects $\left(\mathrm{E}_{\mathrm{max}}\right)$ and sensitivities $\left(\mathrm{pD}_{3}\right)$ of vasoconstrictor and dilator substances in isolated, sympathectomised mesenteric resistance sized arteries from control rats (WKY and SHR) and W'Y rats infused with phenylephrine (WKY'+PHE) or angiotensin II (WKY +AII). $E_{\text {rinax }}$ is expressed in $\mathrm{mN} / \mathrm{mm}$ (vasoconstrictors) or as fraction of the precontraction induced by $30 \mathrm{mM}$ potassium (vasodilators). $\mathrm{PD}_{2}$ and $\mathrm{pCa}_{2}$, represent - $\mathrm{Log}$ Molar $\left[E C_{-1}\right]$. Data are means \pm SEM ( $n=8$ per group). ${ }^{2}$ indicate significance of differences from WKY, SHR or WKY+AII respectively (ANOVA + t-test (Wallenstein, 14R(1) $P<0.05$ ) 


\section{DISCUSSION}

Several changres have been identified in small arteries of adult spontaneously hypertensive rats which may be involved in the development of an elevated peripheral vascular resistance. These include: elevated wall to lumen ratio (Mulvany et al., 1978; Folkow, 1982; Lee, 1987), hyperreactivity to vasoconstrictors (Mulvany et al., 1978; Folkow, 1982), hypersensitivity to vasoconstrictors and calcium (Bolzon and Cheung, 1989; Boonen and De Mey, 1990; Mulvany and Aalkjaer, 1990) and reduced endothelium-dependent relaxing responses (Vanhoutte, 1989; Lüscher, 1990a). The primary or adaptive nature of these alterations remain a matter of debate. Some have been suggested to be genetic in origin (Folkow, 1982) while others may result from increased activity of vasopressor systems or may represent adaptations to elevated transmural pressure (Lever, 1986; Sahlgren et al., 1986; Dostal et al., 1988). The issue of primary causal versus secondary adaptive vascular changes has previously been addressed by developmental studies, the use of long term antihypertensive treatments and by crossbreeding (Aalkjaer et al., 1987; Mulvany, 1988; Christensen et al., 1989; Michel et al., 1992).

We investigated whether pressure elevation in young "normotensive" rats induces vascular changes that are comparable to those in young spontaneously hypertensive rats. Furthermore, we assessed whether these depend upon the pressor agent used. Phenylephrine and angiotensin II were selected in view of i) their pressor and vasoconstrictor actions and ii) suggestions that sympathetic nerves and the renin angiotensin system may participate in the development of hypertension in the spontaneously hypertensive rat (Sahgren et al., 1486; Head, 1989; Resink et al., 1989; de Champlain, 1990). Our findings suggest that structural changes develop earlier in small arteries of spontaneously hypertensive rats than specific functional changes. Herein, angiotensin II may be involved to a larger extent than chronic vasoconstriction, elevated sympathetic vasopressor tone or increased blood pressure (Griffin et al., 1991).

\section{Effects of vasopressor infusion on haemodynamics in young normotensive rats}

In Wistar Kyoto rats, blood pressure triples between birth and adulthood (Gray, 1984). The young Wistar Kyoto rats we used were somewhat in the middle of this developmental phase. Yet, the study period was probably too short to reveal significant increases in systolic blood pressure in these rats. Previously it has been shown that newborn spontaneously hypertensive rats already have an increased blood pressure compared to normotensive controls (Gray, 1984). Other reports indicate that from 6 to 8 weeks of age, blood pressure rises more sharply in spontaneously hypertensive than normotensive rats (Christensen et al., 1989; Morton et al., 1990). The present results confirm this. 6 Week old spontaneously hypertensive rats had a systolic blood pressure that was initially similar to that in age matched Wistar Kyoto rats but that increased significantly within a periud of four days (Fig. 10.2). Infusion of Wistar Kyoto rats with the $\alpha$-adre- 
nergic agonist phenylephrine resulted in an increase in systolic blood pressure of which regression analysis showed a comparable increase compared to that seen in spontaneously hypertensive rats, whereas in Wistar Kyoto rats treated with angiotensin II, systolic blood pressure increased to levels higher than those in spontaneously hypertensive rats (Fig. 10.1 and 10.2).

Because both agents lack major effects on cardiac contractility (Tung et al., 1986; Lindpaintner and Ganten, 1991) and because treatments were accompanied by marked bradycardia (Fig. 10.2), both pressor effects are most likely due to increased peripheral vascular resistance. The increase in peripheral resistance is probably maintained by vasoconstriction and may progressively be taken over by structural changes of the vessel wall (Lever, 1986).

\section{Functional characteristics of mesenteric resistance arteries follo- wing vasopressor infusion in Wistar Kyoto rats}

As alluded above several changes have been observed in arterial smooth muscle of adult spontaneously hypertensive rats which may be responsible for the increased blood pressure (Laher and Triggle, 1983; Mulvany, 1983; Triggle, 1989; Kishi and Inoue, 1990). A hypersensitivity to calcium during pharmacological stimulation has been observed in adult (Triggle and Laher, 1985; Boonen and De Mey, 1990) but also in young spontaneously hypertensive rats in which blood pressure was not yet different from normotensive controls (see: Mulvany and Aalkjaer, 1990). This may indicate that changes in sensitivity of vascular smooth muscle cells of spontaneously hypertensive rats to vasoconstrictors precede the development of blood pressure. Cell cultures of vascular smooth muscle cells of spontaneously hypertensive rats show hypersensitivity to calcium but also to angiotensin II, which strengthens the suggestion that the hypersensitivity is independent of blood pressure and may precede the increase in blood pressure in spontaneously hypertensive rats (Alexander et al., 1990; Bukoski, 1990). The present results, however, indicate that vessels of young spontaneously hypertensive rats, in which blood pressure is significantly increased compared to age matched Wistar Kyoto rats, do not show a hypersensitivity, neither to vasoconstrictors nor to calcium (Table 10.3). They rather suggest that changes in sensitivity to vasoconstrictors in adult spontaneously hypertensive rats may be a consequence rather than a cause of the rise in blood pressure. However, even though differences in sensitivity do not reach statistical significance, it can not be excluded that these small differences, in concert with trophic influences, may play a prominent role in the development of hypertension (Lever, 1986).

Vessels of Wistar Kyoto rats that were made hypertensive through infusion with phenylephrine were, unlike vessels of young spontaneously hypertensive rats, hyposensitive in particular to $\alpha$-adrenergic stimulation with phenylephrine and noradrenaline (in the presence of propranolol). Besides a homologous hyposensitivity, these vessels were hyporeactive to all vasoconstrictor stimuli that were used (Table 10.2 and 10.3). Treatment of Wistar Kyoto rats with angiotensin II, on the other hand showed alterations in vascular reactivity that were comparable to those seen in vessels of young spontaneously hyper- 
tensive rats. No difference in sensitivity to vasoconstrictors could be observed but, like in vessels of young spontaneously hypertensive rats, those of angiotensin treated Wistar Kyoto rats showed a hyperreactivity to vasoconstrictor stimuli (Table 10.3). Although phenylephrine and angiotensin II infusion in Wistar Kyoto rats induce a significant increase in blood pressure, both treatments altered vascular reactivity differently.

From these we conclude that the increase in blood pressure seen in Wistar Kyoto rats infused with angiotensin $I I$ and in spontaneously hypertensive rats could be due to hyperreactivity to vasoconstrictors. Yet, hyperreactivity of small arteries to vasoconstrictors is not essential to maintain an increased blood pressure as is seen in Wistar Kyoto rats treated with phenylephrine. The impaired endothelium-dependent relaxations that were observed in Wistar Kyoto rats treated with both phenylephrine and angiotensin II (Table 10.3) could, in combination with increased vessel tone, also contribute significantly to the maintenance of an increased systolic blood pressure (Vanhoutte, 1989; Lüscher, 1990).

\section{Structural changes in small mesenteric arteries following short term elevation of blood pressure}

To assess whether functional alterations in mesenteric resistance arteries could be explained by changes in vascular structure, vessels, which were fixed at their individual optimal lumen diameter, were subjected to morphometric evaluation of structural parameters. These measurements showed that in vessels of spontaneously hypertensive rats the media cross sectional area was significantly larger than in vessels of Wistar Kyoto rats. As lumen radius was shown to be unchanged, this obviously resulted in an increase in average media thickness (Table 10.2). Consequently, wall to lumen ratio was larger in spontaneously hypertensive rats than in Wistar Kyoto rats (Table 10.2). Previously it has been shown that a slight increase in wall to lumen ratio has important consequences for vessel reactivity (Mulvany et al., 1978; Folkow, 1982). Thus, the observed structural alteration in vessels of young spontaneously hypertensive rats could explain the increased vascular reactivity and vascular resistance.

Surprisingly, in Wistar Kyoto rats treated with phenylephrine, a rise in blood pressure comparable to that seen in spontaneously hypertensive rats, was neither associated with a change in lumen diameter nor a change in media cross sectional area. Therefore, this indicates that an increased blood pressure can be maintained in the absence of structural vascular changes. Even though vessels of Wistar Kyoto rats treated with phenylephrine were hyporeactive and hyposensitive to $\alpha$-adrenergic stimulation, the increased blood pressure is probably due to a vasoconstriction of the resistance vasculature.

Like small arteries of spontaneously hypertensive rats, vessels of Wistar Kyoto rats treated with angiotensin II showed an increased media cross sectional area (Table 10.2). Together with an unchanged lumen diameter this results in an increased average media thickness and wall to lumen ratio (Table 10.2). 
As was the case in vessels of young spontaneously hypertensive rats, the change in small artery structure of Wistar Kyoto rats treated with angiotensin II could explain the hyperreactivity to vasoconstrictors. Both the structural vascular change that is observed and the direct contractile effect of angiotensin II in these vessels could result in the blood pressure rise that is even higher than that in young spontaneously hypertensive rats (Fig. 10.1). The structural vascular changes are either mediated by i) a direct trophic effect of angiotensin Il (Berk et al., 1989a, b; Scott-Burden et al., 1989; Daemen et al., 1991), ii) a central or peripheral effect on sympathetic nerves (Kawasaki et al., 1988; van Kleef et al., 1992), iii) as a result of the increased blood pressure or iv) by a combination of the above. Effects of angiotensin II through a modulatory effect on sympathetic drive, however, are unlikely because of the absence of structural alterations of small arteries in WKY rats treated with phenylephrine. In cell culture, angiotensin II was shown to induce hyperplasia in vascular smooth muscle cells of spontaneously hypertensive rats but not Wistar Kyoto rats (Berk et al., 1989a, b; Scott-Burden et al., 1989). Furthermore, angiotensin Il has also been shown to induce DNA synthesis in arterial smooth muscle in vivo (Daemen et al., 1991; van Kleef et al., 1992). Moreover, studies in which hypertensive rats received antihypertensive treatment with angiotensin converting enzyme (ACE) inhibitors, calcium antagonists, direct vasodilators or $\beta$-adrenoceptor blockers, showed that blood pressure lowering effects of these drugs were comparable. However, following withdrawal of treatment, blood pressure remained lowered only when rats had been treated with ACE-inhibitors but not with the other antihypertensives (Asmar ef al., 1988; Christensen et al., 1989). Measurements of vascular structural parameters suggested that the maintained reduction in blood pressure could be attributed to a structural decrease in wall to lumen ratio in hypertensive rats that had been treated with the ACE-inhibitor perindopril but not with calcium antagonists, $\beta$-blockers or direct vasodilators (Asmar et al, 1988; Christensen et al., 1989; Eriksen et al., 1992).

Taken together, it was shown that short term vasopressor infusion in young normotensive Wistar Kyoto rats resulted in i) induction of resistance artery hypertrophy following angiotensin II but not phenylephrine, ii) homologous hyposensitivity and heterologous hyporeactivity to vasoconstrictors following treatment with phenylephrine iii) reduction of endothelium-dependent relaxing responses and iv) hypertrophy of the heart in Wistar Kyoto rats treated with either angiotensin II and phenylephrine. From these findings we conclude that angiotensin II rather than pressure elevation could be responsible for resistance arterial changes in young spontaneously hypertensive rats. 



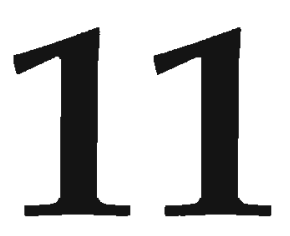

\section{Conclusions and Perspectives}


Small arteries and arterioles determine total vascular resistance (Mulvany and Aalkjaer, 1990; Bohlen and Aukland, 1991). Two major factors are responsible for their modulatory role on blood pressure and flow; their ability to actively constrict and dilate and their overall structural dimensions (Komer et al., 1989; Folkow, 1990; Mulvany and Aalkjaer, 1990). Alterations of these functional and structural parameters may underlie development and maintenance of high blood pressure (Mulvany, 1983; Lee and Smeda, 1985; Lever, 1986; Schmid-Schönbein et al., 1987; Korner et al., 1989). In the present dissertation the involvement of altered reactivity and sensitivity of resistance arterial smooth muscle to vasoactive substances in hypertension was evaluated.

\section{EXCITATION-CONTRACTION COUPLING IN SMALL ARTERIAL SMOOTH MUSCLE}

Contraction and relaxation of vascular smooth muscle are to a considerable extent governed by $\left[\mathrm{Ca}^{2+}\right]$, (Van Breemen et al., 1986; Carafol, 1987; Hai and Murphy, 1989). Calcium can be mobilised following depolarisation or pharmacological stimulation of smooth muscle through separate sets of calcium channels or from intracellular stores (see Chapter 1). Depolarisation of vascular smooth muscle activates voltage operated calcium channels leading to the influx of calcium, whereas pharmacological stimulation is thought to not only open calcium channels but additionally induce the release of calcium from intracellular stores and possibly modify the calcium-sensitivity of intracellular control elements (Sumlyo and Somlyo, 1968; Berridge, 1986c; Van Breemen et al., 1486). These means of activation of the contractile apparatus are referred to as electro-mechanical and pharmacomechanical coupling, respectively (Somlyo and Somlyo, 1968; Droogmans et al., 1977; Van Breemen et al., 1986; Mulvany and Aalkjaer, 1990).

\section{$\alpha_{1}$-adrenoceptor heterogeneity in small and large mesenteric arteries}

Judging from the affinity for the $\alpha_{1}$-adrenoceptor antagonist WB 4101 and from the actions of the non-competitive $\alpha_{1}$-adrenoceptor antagonist chloroethylclonidine, subtypes of $\alpha_{1}$-adrenoceptors were found to be heterogeneously distributed over large and small mesenteric arteries of the rat (Chapter 3). Functionally, responses of large mesenteric arteries to noradrenaline can be referred to as primarily $\alpha_{18}$-adrenoceptor-mediated whereas those of small mesenteric arteries display characteristics that are in agreement with the activation of putative $\alpha_{1 A}$-adrenocepturs (Han et al., 1987; Minneman, 1988; Oriowo and Ruffolo, 1992). Consequently, contractile responses induced by noradrenaline in large arteries rely more on the metabolism of phosphatidylinositol than in small arteries (Berridge, 1980d, c; Van Breemen and Saida, 1989). This heterogenous distribution of $\alpha_{1}$ adrencceptor subtypes could explain the less profound inhibitory effect of calcium antagonists on contractions induced by noradrenaline in large than those in small mesenteric arteries. However, keeping in mind the proposed 
existence of different sets of calcium channels in vascular smooth muscle (Bolton, 1979; Hurwitz, 1986; Bean, 1989), a problem arises.

\section{Different calcium channels in small artery smooth muscle?}

Receptor operated calcium channels are believed to be rather refractory to inhibition by calcium antagonists (Godfraind and Kaba, 1972; Cauvin et al., 1983; Fleckenstein et al., 1984; Julou and Freslon, 1986; Bean, 1989). Yet, contractile responses induced by depolarisation but also those to noradrenaline are inhibited equally well by the dihydropyridine calcium antagonist felodipine in large mesenteric arteries (Chapter 3). Furthermore, even though the $\alpha_{1 \mathrm{~A}}$-adrenoceptors, that mediate responses to noradrenaline in small arteries, have been proposed to be directly coupled to receptor-operated calcium channels (Han et al., 1987; Nichols et al., 1989; Wilson and Minneman, 1990), the affinity for felodipine is even larger in small than in large arteries (Nyborg and Mulvany, 1984; Nordlander, 1989; Ljung, 1990; Messing et al., 1991; see also Chapter 3). As dihydropyridine calcium antagonists have been shown to be highly selective in affecting the activity of L-type voltage operated calcium channels (Godfraind and Kaba, 1972; Fleckenstein et al., 1984; Schwartz, 1989; Nelson et al., 1990), this suggests that in small arteries the contribution of voltage operated calcium channels in the contractile response to pharmacological stimulation is high. Several reports indicate that pharmacological stimulation of small arteries activates not only receptor operated calcium channels but indirectly also voltage operated calcium channels through a depolarising effect of noradrenaline (Mulvany et al., 1982; Cauvin and Van Breemen, 1985; Nelson et al., 1990; see also Chapter 8 ).

\section{Noradrenaline affects the function of voltage operated calcium channels}

Besides the above mentioned classical explanations, I prefer the following alternative: noradrenaline induces influx of calcium in small arteries through direct activation of voltage operated calcium channels (Fig 11.1). Numerous observations are in agreement with this hypothesis. Firstly, in vascular smooth muscle cells isolated from small arteries it was shown by electrophysiological experiments that noradrenaline directly affects the open probability of voltage operated calcium channels by shifting the voltage dependence of the channels to more negative membrane potentials (Benham and Tsien, 1988; Nelson et al., 1988). Secondly, receptor-operated calcium channels activated by noradrenaline have never been identified in vascular smooth muscle by electrophysiological techniques. Results presented in chapter 4 suggest that the sensitivity for extracellular calcium is higher in vessels stimulated with noradrenaline than in those exposed to high potassium, which could be explained by effects of noradrenaline on superficial calcium barriers (Saida and Van Breemen, 1984; Van Breemen et al., 1986) or on the calcium sensitivity of the contractile apparatus (Nishimura et al., 1988; Himpens et al., 1990; Jensen et al., 1992). However, in the presence of both potassium plus noradrenaline, contractile responses can be elicited in small arteries by calcium concentrations that are below the threshold to induce a contractile response in the presence of either stimulus alone, suggesting that noradrenaline and potassium interact synergistically at a common site 
(Chapter 4). Although indirectly, this is in agreement with the hypothesis that noradrenaline activates voltage operated calcium channels.

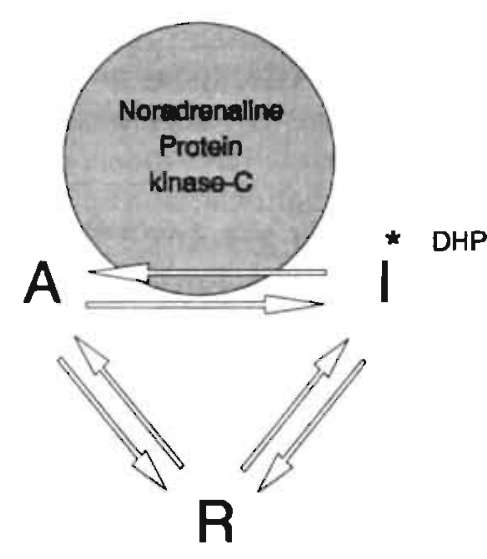

The three state calcium channel model

The characteristics of the contractile response to potassium could be explained by a three state voltage operated calcium channel (Chapter 4) as it was previously described in cardiac muscle (Hess et al., 1984; Bean et al., 1986). At rest (polarised conditions), voltage operated calcium channels would preferentially remain in a resting, closed state. The probability of the channels to reside in an activated, open state increases with increasing depolarisation of the cellmembrane (Fig. 11.1). In depolarised preparations, the calcium channel would switch between the activated, open state and an inactivated closed state (Fig. 11.1). Dihydropyridines would preferentially bind to the inactivated state leading either to full inhibition of the channel or full activation (Hess et al., 1984; Bean et al., 1980), depending on the antagonistic or agonistic properties of the drug. Noradrenaline may shift the equilibrium between the inactive and active state towards the active state of the channel (Fig. 11.1) (Nelson et al., 1990). Upon repolarisation of the cellmembrane, the open probability of the channels decreases again and the channels remain most of the time in the resting state.

\section{Hypersensitivity to calcium in small arteries of $S H R$}

As was shown previously (Mulvany and Nyborg, 1980; Aqel et al., 1986; Bolzon and Cheung. 1989), results in chapter 4 confirm that in small arteries of adult SHR, the sensitivity for extracellular calcium is significantly increased during $\alpha$-adrener- 
gic but not electro-mechanical stimulation. The dihydropyridine nimodipine abolishes this difference (Chapter 4) which is in agreement with the above mentioned hypothesis. Furthermore, rhythmic responses that could only be observed in small mesenteric arteries of SHR during stimulation with noradrenaline could be mimicked by the dihydropyridine calcium agonist Bay K8644 in vessels of WKY rats (Chapter 4). Rhythmicity of contractile responses in a multicellular preparation implies cellular communication (conduction) and synchronisation (pacemaker activity), and modulatory roles of noradrenaline and dihydropyridines of this oscillatory behaviour suggest the involvement of voltage operated calcium channels (Chapter 4). Taken together, these findings led us to suggest that small arteries of WKY and SHR posses qualitatively similar mechanisms of excitation-contraction coupling but that in the latter, the modulatory effect of noradrenaline on voltage operated calcium channels is altered (Fig. 11.1). This effect may be mediated through the effects of second messengers. Observations by others support this statement. The activity of phospholipase- $C$ which is believed to be involved in $\alpha$-adrenergic stimulation, has been shown to be increased in platelets of genetically hypertensive rats (Koutouzov et al., 1987). Furthermore, the activity of protein kinase-C, which is stimulated following activation of phospholipase-C has been shown to be enhanced in vascular smooth muscle of SHR (Uehara et al., 1988). Yet, it can not be excluded that the observed hypersensitivity to calcium in smooth muscle of SHR is due to alterations inherent to the voltage operated calcium channels themselves or to altered modulation by noradrenaline of the calcium-sensitivity of intracellular regulatory elements (Sturek and Hermsmeyer, 1986; Hermsmeyer, 1987). Nonetheless, the question arose how noradrenaline could affect the function of voltage operated calcium channels?

\section{The involvement of G-proteins in the contractile response to agonists}

In many cell types it was shown that ion channels may be coupled to GTP-binding regulatory proteins (G-proteins) (Gilman, 1987; Brown and Birnbaumer, 1988; Birnbaumer, 1990). Various G-protein subtypes can be distinguished according to their substrates and sensitivity to bacterial toxins (Gilman, 1987; Brown and Birnbaumer, 1988; Birnbaumer, 1990). Pertussis toxin has been shown to selectively inactivate a subset of $\mathrm{G}$-proteins $\left(\mathrm{G}_{1}\right.$ and $\mathrm{G}_{0}$-proteins) (Graziano and Gilman, 1987; Casey and Gilman, 1988). From experiments in vivo it was suggested that $\alpha_{2}$-adrenoceptors on rat vascular smooth muscle were coupled to calcium channels through pertussis toxin sensitive G-proteins, whereas $\alpha_{1}$-adrenoceptors were either coupled directly to calcium channels through pertussis toxin sensitive G-proteins or to intracellular release of calcium through pertussis toxin insensitive Gproteins (Nichols et al., 1989).

Our In vitro experiments on isolated small mesenteric arteries showed that the influx of calcium following $\alpha_{1}$-adrenergic stimulation was sensitive to pertussis toxin, suggesting the involvement of a pertussis toxin sensitive Gprotein in the coupling of $\alpha_{1}$-adrenoceptors to calcium channels (Chapter 5). Taking into account the findings in chapter 3 , this indicates that $\alpha_{1 \mathrm{~A}}$-adrenoceptors on resistance arterial smooth muscle couple through a pertussis toxin sen- 


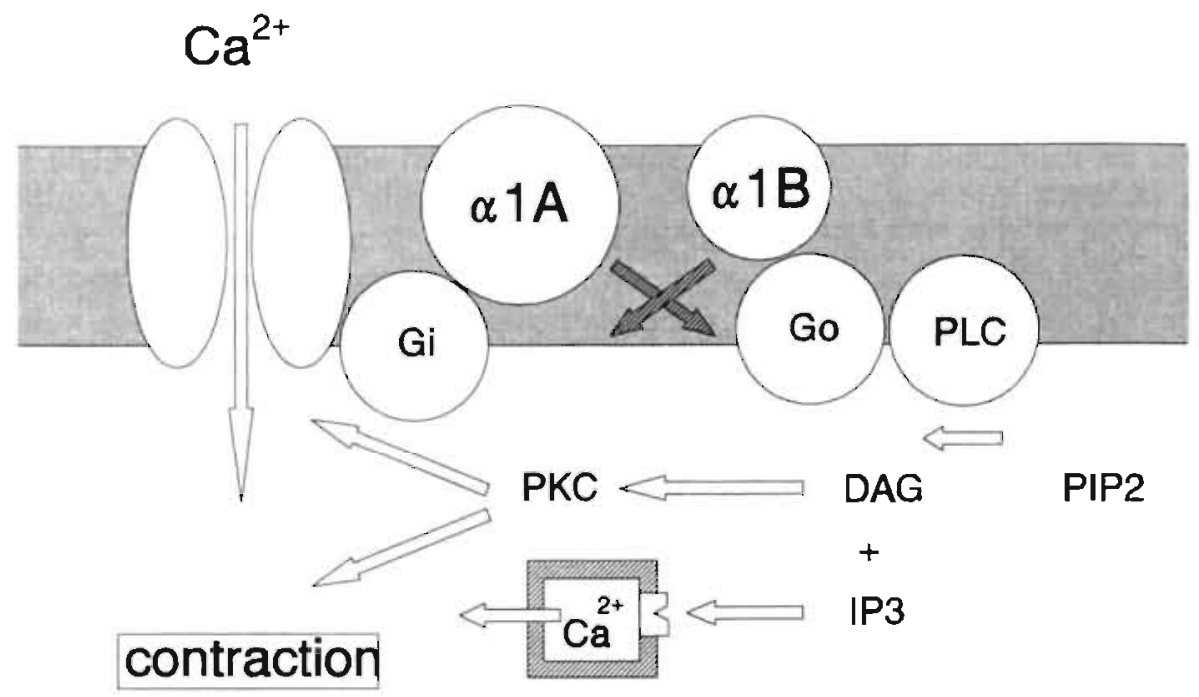

Figure 11.2. Schematic representation of a small artery smooth muscle cell membrane. Both $\alpha_{1 A}$ and $\alpha_{1 B}$-adrenoceptors were identified. The relative contribution of these $\alpha_{1}$-adrenoceptors in small artery smooth muscle is larger for the $\alpha_{1 A^{-}}$then the $\alpha_{1 \mathrm{~B}}$-subtype (indicated by size of receptor). $\alpha_{14}$-adrenoceptors may couple to a calcium channel through pertussis toxin sensitive $G$ proteins $\left(G_{i}\right.$ ). whereas $\alpha_{1 B}$-adrenoceptors couple through a pertussis toxin sensitive $G$-protein $\left(G_{0}\right)$ to phospholipase-C (PLC). Activation of PLC leads to the generation of inositol triphosphate $\left(\mathrm{IP}_{3}\right)$ and diacylglycerol (DAG). $\mathrm{IP}_{3}$ stimulates the release of calcium from intracellular stores. Diacylglycerol activates protein kinase-C (PKC) which in turn may affect the calcium sensitivity of the contractile apparatus or the function of calcium channels. Both $\alpha_{1}$-adrenoceptor subtypes may, however, couple to other $G$-proteins (crossed arrows), suggesting that $\alpha_{1 A}$-adrenoceptor activation may stimulate PLC and $\alpha_{18}$-adrenoceptor activation may lead to calcium channel activation.

sitive G-protein to calcium channels (Fig. 11.2). However, unlike observations by Nichols et al., (1989), also the contractile response induced by the $\alpha_{1}$-adrenoceptor agonist phenylephrine in the absence of extracellular calcium was abolished following pretreatment with pertussis toxin. Moreover, the inhibition of contractile responses to noradrenaline in the absence of extracellular calcium by pretreatment with chloroethylclonidine suggests that these adrenoceptors belong to the $\alpha_{18}$-adrenoceptor subtype which are thought to couple to phospholipase-C (Fig. 11.2) (Chiu et al., 1987; Khalil et al., 1987; Raymond et al., 1990). Paradoxically, the G-proteins that couple to phospholipase- $C$ have in several studies been suggested to be insensitive to inactivation by pertussis toxin (Nichols et al., 1989). This could indicate that $\alpha_{1}$-adrenoceptor subtypes are not necessarily linked to specific $\mathrm{G}$-proteins but that these receptors may also activate other G-proteins 
(Fig. 11.2) (Liebau et al, 1989). Similarly, serotonergic $\left(5 \mathrm{HT}_{2}\right)$-, vasopressin $\left(\mathrm{V}_{1}\right)$ and endothelin $\left(E T_{1}\right)$-receptors which have also been shown to activate phospholipase-C (Grillone et al., 1988; Minneman, 1988; Akbar et al., 1989; Huang et al., 1989; Kondo et al., 1989; Mitsuhashi et al., 1989) may have a different affinity for specific G-proteins and hence for the activation of the G-protein coupled second messenger systems (Chapter 5). In analogy with the suggestion proposed by Liebau et al., (1989), agonist-receptor complexes may have different affinities for specific Gproteins (Fig. 11.2), leading to different "intrinsic activities" and relative resistance to inhibitors of second messenger systems.

\section{Does protein kinase-C play a role in contraction of small arteries?}

Activation of phospholipase- $C$ leads to the generation of two second messengers; inositol triphosphate $\left(\mathrm{IP}_{3}\right.$ ) and diacylglycerol (DAG) (Berridge, 1986a). The former induces the release of calcium from intracellular stores (Berridge, 1986b) and is believed to be involved in the initiation of the contractile response. Observations in chapters 3 and 5 are in accordance with this. In the maintenance of the contractile response however, $\mathrm{IP}_{3}$ appears to play a minor role which is strengthened by the observation that chloroethylclonidine is rather ineffective in reducing contractile responses to noradrenaline in the presence of calcium in small mesenteric arteries (Chapter 3). DAG is a potent activator of protein kinase-C (Nishizuka, 1986). This kinase is believed to exert multiple effects on ion handling, and intracellular mechanisms (Huganir and Greengard, 1987; Rasmussen et al., 1987; Fabbro et al., 1988; Khalil and Van Breemen, 1988; Drenth et al., 1989; Boscoboinik et al., 1990; Raymond et al., 1990; see also Chapter 6).

We evaluated the role of protein kinase- $\mathrm{C}$ in contractile responses of small arteries. Activation of protein kinase- $C$ by phorbol myristate acetate (Nishizuka, 1986) caused a significant shift in the sensitivity for the contractile effect of potassium (Chapter 6). Furthermore, staurosporine, an inhibitor of protein kinase- $C$ activity, reduced contractile responses to high potassium (Chapter 6). These findings suggest that protein kinase- $C$ is involved in activation of voltage operated calcium channels. Following depolarisation of vascular smooth muscle, protein kinase- $C$ may be activated by calcium entering the cell through voltage operated calcium channels and in turn, protein kinase- $\mathrm{C}$ may further activate calcium influx (Berridge, 1986b; Nishizuka, 1986; Ho et al., 1988). Whether this is through a direct effect of protein kinase-C on the calcium channel (Fish et al., 1988) or through effects on membrane potential remains to be established. Besides an effect of protein kinase-C on membrane channels, the kinase exerted effects on intracellular regulatory elements rendering the contractile machinery more sensitive to calcium. The latter effect was demonstrated previously by Drenth et al., (1989) and Nishimura et al., (1990). These authors suggested that protein kinase-C was responsible for the increased sensitivity of the contractile apparatus for calcium during $\alpha_{1}$-adrenergic stimulation. Taken together, the results indicate that protein kinase-C is probably not only involved in contractile responses of resistance arterial smooth muscle following pharmacological stimulation but also during electro-mechanical coupling (Fig. 11.3). 
Modulation of contractile responses in small arteries by mechanical factors

As discussed in chapters 7 and 8, isometric contractile responses in isolated small mesenteric arteries are sensitive to modulation by distension. A length-dependency of contractile responses has been described in striated muscle but also in vascular smooth muscle and was attributed to overlap of contractile filaments (Gordon et al., 1966; Mulvany and Warshaw, 1979). However, findings presented in Chapter 7 do not only show a non-selective length-dependency of the amplitude of contractile responses, but also "agonist" selective effects on sensitivity and effects on the morphology of responses to high potassium which we suspect are linked to the three state mode of activation of voltage operated calcium channels.

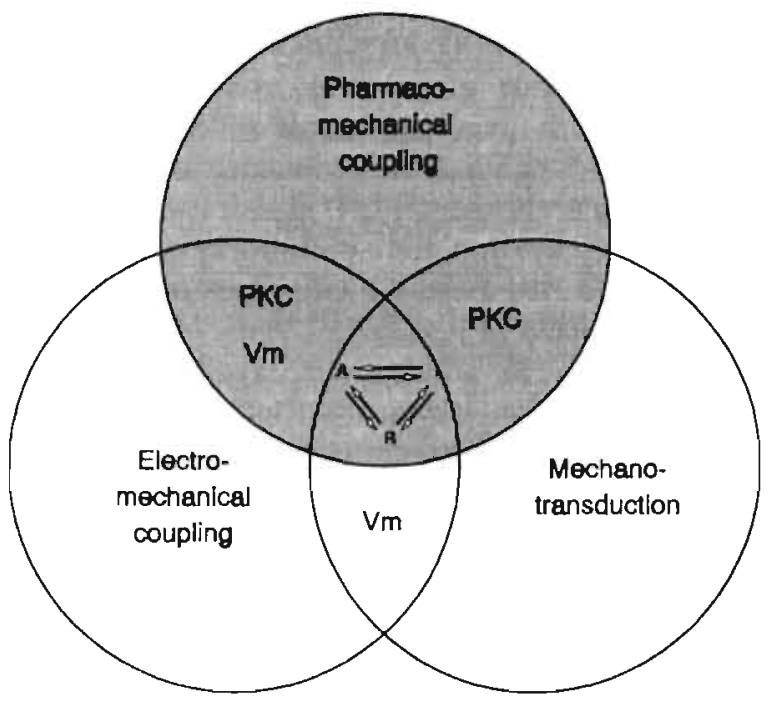

Figure 11.3. Excitation-contraction coupling in small artery smooth muscle represented as the three major pathways that can be distinguished: electromechanical coupling, pharmacomechanical coupling and mechanotransduction. In small arteries these operate in close relation to each other to regulate the function of voltage operated calcium charnels (middle) through modulation of the excitability of the cell membrane $\left(\mathrm{V}_{\mathrm{m}}\right)$ or through the activity of protein kinase-C (PKC). In adult SHR a change may be present within the shaded area resulting in hypersensitivity to vasoconstrictors.

We further investigated the involvement of calcium channels in the distension dependency of contractile responses in isolated small arteries (Chapters 7 and 8 ). The sensitivity for depolarising solution, $\alpha_{1}$-adrenergic stimulation and extracellular calcium increased with increasing distension of small arteries. These observations confirm those made previously by Price et al., (1981) and Nilsson and Sjöblom, (1985). The hypersensitivity for potassium as a result of distension was not affected by the removal of the endothelium, suggesting that in this respect, the modulatory role of the endothelium on vascular smooth 
muscle function is minor (Griffith and Edwards, 1990; Rubanyi et al., 1990). Indirect observations presented in Chapter 7 suggest that distension could affect the regulation of membrane potential of small artery smooth muscle and additionally affect the sensitivity of the contractile apparatus for calcium. Direct measurements of membrane potential confirmed these suggestions (Chapter 8). At rest, small artery smooth muscle cells are less polarised in highly distended vessels as compared to those with low distension. This difference persisted in the presence of intermediate stimulations with potassium. As a consequence $\left[\mathrm{Ca}^{2+}\right]_{\mathrm{i}}$ is increased during intermediate stimulation of vessels at high distension. This could partly explain the hypersensitivity to potassium and noradrenaline during distension. However, besides a change in membrane potential, the sensitivity of the contractile apparatus for calcium was observed to be increased with distension (Chapter 8) suggesting that the efficacy of the contractile response increases with higher distension. It remains to be elucidated how distension alters membrane potential and the sensitivity of the contractile apparatus for calcium. In this respect, stretch sensitive ion-channels (Laher and Bevan, 1989; Meininger et al., 1991a) or activation of second messenger systems (Watson, 1991) may be involved.

\section{Protein kinase-C and mechanotransduction}

Effects of activation of protein kinase-C (Chapter 6) and distension (Chapters 7 and 8) on contractile responses induced by depolarising potassium solution show a marked similarity. Both activation of protein kinase- $\mathrm{C}$ and distension of small arteries increase the sensitivity of the contractile effect of potassium. One may speculate that a relation exists between the activity of protein kinase- $C$ and distension-dependent modulation of contractile responses following electro-mechanical stimulation. Some reports are in agreement with this hypothesis. It has been shown that in striated muscle, protein kinase- $C$ can be activated by deformation of the cell (Richert et al., 1987; Watson, 1991). Also in stretch-induced responses in isolated small arteries, protein kinase-C has been suggested to play an important role (Laher et al., 1989; Osol et al., 1991). Additionally, calcium influx in itself may trigger phospholipase- $C$ activity and subsequently activate protein kinase-C (Berridge, 1986b; Nishizuka, 1986; Ho et al., 1988) which in turn may promote calcium entry in a positive feedback manner. However, Ohanian and Heagerty, (1991) and Ollerenshaw et al., (1991) suggested that the role of protein kinase- $C$ in vascular reactivity would be negligible because they showed that diacylglycerol, which is an activator of protein kinase-C, is metabolised very rapidly into posphatidic acid and arachidonic acid. Activation of protein kinase-C, however, may require the presence of physical stress (Laher and Bevan, 1987; Laher et al., 1989; Hill et al., 1990; Osol et al., 1991) or an intact integrity of cytoskeletal components (Richert et al., 1987; Watson, 1991) which were absent in these biochemical studies. Nonetheless, it is unclear whether effects of dynamic-stretch (myogenic autoregulatory responses) and static stretch (increased mean blood pressure) on excitation-contraction coupling can be ascribed to the same mechanotransducing mechanism. Therefore, finding the physical parameter responsible for distension-induced changes in excitation-contraction coupling is 
crucial to the understanding of these phenomena. Inseparable physical factors such as pressure, tension, strain, stress or dynamic stretch, however, complicate the analysis of the true sensors in mechanotransducing pathways.

Regardless the nature of the length- or stretch sensor, differences are present in effects of distension on contractile responses in small arteries of WKY and SHR. The relation between transient and sustained responses to potassium was significantly different between vessels of these strains. It is tempting to speculate that the altered sensitivity for calcium that is seen during $\alpha_{1}$-adrenergic stimulation, rhythmic contractile responses in small arteries and differences in length-dependency of small arteries of SHR can be explained by the altered activity of the phosphoinositide signalling pathway (Koutouzov et al., 1987; Ui'hara et al., 1988). Moreover, observations with respect to mechanotransducing mechanisms are in line with the hypothesis put forward for the role of protein kinase-C in electromechanical and pharmacomechanical coupling. In conclusion the above findings may suggest that on the resistance arterial level electro-mechanical coupling, pharmacomechanical coupling and mechanotransduction act in concert rather than separately to affect small artery reactivity (Fig. 11.3).

\section{SMALL ARTERY REACTIVITY AND HYPERTENSION}

Do changes in vascular reactivity contribute to the development or maintenance of hypertension? The answer to this question was the objective of a vast array of studies in essentially hypertensive humans and various experimental animal models of hypertension. Many reports show a hypersensitivity of vascular smooth muscle to vasoconstrictors in hypertensive subjects (Lais and Brody, 1978; Webb, 1984; Triggle, 1989; Kong et al., 1991). Hypersensitivity to vasoconstrictors has been suggested to reside in a cellular defect in the handling of calcium (Harder and Hermsmeyer, 1983; Hermsmeyer and Harder, 1986; Exton, 1988; Sharma and Bhalla, 1988; Swales, 1990), which has been shown to be present in the absence of an increased blood pressure (Mulvany and Nyborg. 1980; Bukoski, 1990) and was therefore put forward as a possible candidate involved in the genesis of hypertension. On the contrary, other observations in small arteries of hypertensive humans show a hyposensitivity to the contractile effect of calcium (Aalkjaer and Mulvany, 1983; Aalkjaer et al., 1987).

Changes in receptor-effector coupling could be responsible for hypersensitivity to vasoconstrictors. In this respect, alterations in adrenoceptor affinity (Michel et al., 1989, 1990), changes in the activity of phospholipase-C (Koutouzov et al., 1987; Uehara et al., 1988), and changes in the excitability of the cellmembrane (Harder and Hermsneyer, 1983; Hermsmeyer, 1987) have been suggested. On a higher level, hyperactivity of the sympathetic nervous system (Abel and Hermsmeyer, 1981; Abboud, 1982; Head, 1989; de Champlain, 1990; Esler et al., 1990) and the renin-angiotensinsystem (Lüscher, 1990; Scott-Burden et al., 1992) have been suggested to be responsible 
for the development of hypertension. Changes in vascular structure that may either be genetically determined (Folkow et al., 1958; Folkow, 1982; Gray, 1984) or brought about by any of the above mentioned alterations (Overbeck, 1980; Lever, 1986; Plunkett and Overbeck, 1988; Smeda et al., 1988; Daemen et al., 1991; Schelling et al., 1991), could play a pivotal role in the evolution of the hypertensive state.

\section{Tissue culture of large and small mesenteric arteries}

To evaluate the primary or secondary nature of changes in vascular reactivity and their relation to high blood pressure, two approaches were used. Tissue culture of isolated mesenteric arteries was chosen because this offers some advantages compared to in vivo studies or cultures of isolated smooth muscle cells. Vascular reactivity and sensitivity to vasoconstrictors could be assessed in the absence of pressure and pressor influences of neuro-endocrine origin. It was shown that qualitative differences in sensitivity and reactivity to different vasoconstrictors exist in small and large mesenteric arteries of adult SHR. Small arteries were hypersensitive to phenylephrine whereas large arteries were hypersensitive to both serotonin and vasopressin. These differences were lost during four day culture (Chapter 9), suggesting that hypersensitivity to vasoconstrictors in hypertension could be a secondary adaptation.

\section{Vascular reactivity in young normotensive and hypertensive rats}

In 6 week old SHR systolic blood pressure is slightly increased compared to that in WKY (Chapter 10). Vascular sensitivity for different vasoconstrictors was unaltered (Chapter 10). These observations suggest that it is unlikely that the hypersensitivity for vasoconstrictors observed in adult SHR is a primary defect. They therefore strengthen the hypothesis that hypersensitivity may be secondary to blood pressure rise. A hypersensitivity for calcium during $\alpha_{1}$-adrenergic stimulation has, however, previously been observed in both adult (Mulvany and Nyborg, 1980) and young SHR in which blood pressure was only marginally elevated (Mulvany and Nyborg, 1980). The present results do not confirm these latter findings (Chapter 10). Small arteries of young SHR did, however, show a nonselective hyperreactivity to vasoconstrictors, which could be explained by increased media cross-sectional area (Chapter 10). These changes in vascular structure could be inherent to SHR and independent of the increased blood pressure. To evaluate this possibility we infused young WKY rats with phenylephrine or angiotensin II. These substances were chosen because they mimic overactivity of systems that have been suggested to be involved in the development of hypertension (Abel and Hermsmeyer, 1981; Abboud, 1982; Head, 1989; de Champlain, 1990; Esler et al., 1990; Lüscher, 1990; Scott-Burden et al., 1992).

Unlike small mesenteric arteries of SHR those of WKY treated with phenylephrine displayed a general hyporeactivity to vasoconstrictors and a homologous hyposensitivity to $\alpha$-adrenergic stimulation. Treatment of WKY with phenylephrine did not alter structural dimensions of these small arteries. In combination with only minor effects of phenylephrine on cardiac contractility (Tung et al., 1986), marked bradycardia and reduced endothelium-dependent relaxing reactivity, this suggests that the elevated blood pressure was probably due 
to increased active vasoconstriction. Like in WKY treated with phenylephrine, angiotensin II caused significant bradycardia and reduced endothelium-dependent relaxing responses, suggesting that the increase in blood pressure was also due to elevated peripheral vascular resistance. In contrast to phenylephrine, vascular reactivity was shown to be increased significantly following infusion with angiotensin II (Chapter 10). Like in vessels of young SHR, this change in reactivity was accompanied by a marked increase in media cross sectional area and wall to lumen ratio. From these, we conclude that angiotensin II rather than an elevated blood pressure may be responsible for small artery changes in young spontaneously hypertensive rats. Angiotensin II may affect vascular structure through a direct trophic effect on vascular smooth muscle (Daemen et al., 1491, (iriftin et al., 1991), a central or peripheral effect on sympathetic neurotransmission (Kawasaki et al., 1988; van Kleef et al., 1992) or through a combination of these. The role of the renin-angiotensin system in the pathogenesis of hypertension in spontaneously hypertensive rats is strengthened by observations that long term administration of ACE-inhibitors, unlike antihypertensive treatments with calcium antagonists, vasodilators or $\beta$-blockers, prevent and reverse structural vascular changes (Christensen et al., 1989; Eriksen et al., 1992). The discrepancy between this and the normal plasma renin activity in spontaneously hypertensive rats may find its origin in altered tissue renin-angiotensin system and increased structural responsiveness of arterial smooth muscle cells of spontaneously hypertensive rats to normal levels of angiotensin II.

\section{Conchusions and perspectioes}

From the present thesis it can be concluded that different mechanisms of excitation-contraction coupling exist in small artery smooth muscle. These mechanisms interdigitate in many aspects. In this regard, it is suggested that membrane potential and protein kinase-C play important roles in the activation of voltage operated calcium channels and contraction of small artery smooth muscle by electrical, pharmacological as well as mechanical stimuli. Differences in the activity of in particular the phosphoinositide system may explain changes in vascular reactivity that are observed in adult spontaneously hypertensive rats. Vascular reactivity, however, is governed to an important extent by vascular structural dimensions. Changes in vascular structure rather than in hypersensitivity for vasoconstrictors or calcium or hyposensitivity to vasodilators may be responsible for the development of the increased blood pressure in spontaneously hypertensive rats. Antihypertensive agents that not only lower blood pressure through vasodilatation of resistance arterial smooth muscle but also through regression of altered vascular structure would therefore be favourable for successfull treatment of hypertension. Because not only elevated blood pressure but also structural vascular changes start to be recognised as independent risk factors for stroke, coronary artery disease and myocardial infarction, this consideration may have consequences for human morbidity and mortality. 


\section{REFERENCES}

Aalkjaer C. and M. Mulvany, 1983, Human and rat resistance vessels: a comparison of their morphological and pharmacological characteristics, Gen. Pharmacol, 14: 85-87.

Aalkjaer C., A.M. Heagerty, 1. Bailey, M.J. Mulvany and J.D. Swales, 1987a, Studies of isolated resistance vessels from offspring of essential hypertensive patients, Hypertension., 9 . III $155-$ IIII 158.

Aalkjaer C., A.M. Heagerty and M.J. Mulvany, 1987b, Evidence for a negative correlation between verapamil sensitivity and calcium sensitivity in isolated human resistance vessels, J. Hypertension., 5: S157-\$159.

Aalkjaer C., A.M. Heagerty, K.K. Petersen, J.D. Swales and M.J. Mulvany, 1987c, Evidence for increased media thickness, increased neuronal uptake, and depressed excitation-contraction coupling in isolated resistance vessels from essential hypertensives, Circ. Res., 61: 181-186.

Abboud F.M., 1982, The sympathetic system in hypertension, Hypertension., 4: 11208-I1225.

Abel P.W. and K. Hermsmeyer, 1981, Sympathetic cross-innervation of SHR and genetic controls suggests a trophic influence on vascular muscle membranes, Circ. Res., 49: $1311-1318$.

Adelstein R.S. and J.R. Sellers, 1987, Effects of calcium on vascular smooth muscle contraction, Am. J. Cardiol., 59: 4B-10B.

Akbar H., N.Y. Chen, D.V. Fossen and D. Wallace, 1989, Increased vascular contractile sensitivity to serotonin in spontaneously hypertensive rats is linked with increased turnover of phosphoinositide, Life. Sci., 45: 577-583.

Alexander D., J.P. Gardner, H. Tomonari, B.P. Fine and A. Aviv, 1990, Lower $\mathrm{Na}^{*}-\mathrm{H}^{+}$antiport activity in vascular smooth muscle cells of Wistar-Kyoto rats than spontaneously hypertensive and Wistar rats, J. Hypertension., 8: 867-871.

Aprigliano O. and K. Hermsmeyer, 1977, Trophic influences of the sympathetic nervous system on the rat portal vein, Circ. Res., 41: 198-206.

Aqel M.B., R.V. Sharma and R.C. Bhalla, 1986, Increased $\mathrm{Ca}^{2+}$ sensitivity of $\alpha_{1}$-adrenoceptorstimulated contraction in SHR caudal artery, Am. J. Physiol, 250: C275-C282.

Aqel M.B., R.V. Sharma and R.C. Bhalla, 1987, Increased norepinephrine sensitive intracellular $\mathrm{Ca}^{2+}$ pool in the caudal artery of spontaneously hypertensive rats, J. Hypertension., 5: 249-253.

Artalejo C.R., M.A. Ariano, R.L. Perlmann and A.P. Fox, 1990, Activation of facilitation calcium channels in chromaffin cells by D1 dopamine receptors through a CAMP/protein kinase-A dependent mechanism, Nature., 348: 239-242.

Asano M., K. Aoki, Y. Suzuki and T. Matsuda, 1987, Effects of Bay K8644 on isolated dog cerebral, coronary and mesenteric arteries, J. Pharmacol. Exp. Therap., 647: 646-656.

Asano M., K. Masuzawa, T. Matsuda and T. Asano, 1988, Reduced function of the stimulatory GTP-binding protein in $\beta$-adrenoceptor-adenylate cyclase system of femoral arteries isolated from spontaneously hypertensive rats, J. Pharmacol. Exp. Therap., 246: 709-718.

Asmar R.G., B. Pannier, J.P.H. Santoni, S.T. Laurent, G.M. London, B.I. Levy and M.E. Safar, 1988, Reversion of cardiac hypertrophy and reduced arterial compliance after converting enzyme inhibition in essential hypertension, Circ. Res., 78: 941-950.

Barany K., A. Rokolya and M. Barany, 1990, Stretch activates myosin light chain kinase in arterial smooth muscle, Biochem. Biophys. Res. Commun., 173: 164-171.

Bayliss W.M., 1902, On the local reactions of the arterial wall to changes of internal pressure, J. Physiol, 28: 220-231.

Bean B.P., 1989, Classes of calcium channels in vertebrate cells, Ann. Rev. Physiol., 51: 367-384.

Bean B.P., M. Sturek, A. Puga and K. Hermsmeyer, 1985, Calcium channels in smooth muscle cells from mesenteric arteries, J. Gen. Physiol., 86: 23.

Bean B.P., M. Sturek, A. Puga and K. Hermsmeyer, 1986, Calcium channels in muscle cells isolated from rat mesenteric arteries: modulation by dihydropyridine drugs, Circ. Res., 59 : $229-235$.

Benham C.D. and R.W. Tsien, 1987, A novel receptor-operated $\mathrm{Ca}^{2+}$-permeable channel activated by ATP in smooth muscle, Nature., 328: 275-278. 


\section{REFERENCES}

Benham C.D. and R.W. Tsien, 1988, Noradrenaline modulation of calcium channels in single smooth muscle cells from rabbit ear artery, J. Physiol., 404: 767-784.

Berk B.C., A. Brock, R.C. Webb, M.B. Taubman, W.J. Atkinson, M.A. Gimbrone and R.W. Alexander, 1985, Epidermal-growth-factor, a vascular-smooth-muscle mitogen, induces rat aortic contraction, J. Clin. Invest., 75: 1083-1086.

Berk B.C., G. Vallega, A.J. Muslin, H.M. Gordon, M. Canessa and W. Alexander, 1989a, Spontaneously hypertensive rat vascular smooth muscle cells in culture exhibit increased growth and $\mathrm{Na}^{*} / \mathrm{H}^{*}$ exchange, J. Clin. Invest., 83: 822-829.

Berk B.C., V. Vekshtein, H.M. Gordon and T. Tsuda, 1989b, Angiotensin II-stimulated protein synthesis in cultured vascular smooth muscle cells, Hypertension., 13: 305-314.

Berridge M.J., 1986a, Cell signalling through phospholipid metabolism, J. Cell. Sci., S4: 137-153.

Berridge M.J., 1986b, Inositol triphosphate and calcium mobilization, J. Cardiovasc. Pharmacol., 8: S85-S90.

Berridge M.J., 1986c, Intracellular signalling through inositol triphosphate and diacylglycerol, Biol. Chem., 367: 447-456.

Berridge M.J. and A. Galione, 1988, Cytosolic calcium oscillators, Faseb. J., 2: 3074-3082.

Berridge M.J. and R.F. Irvine, 1984, Inositol triphosphate, a novel second messenger in cellular signal transduction, Nature., 312: 315-321.

Bers D.M. and J.H.B. Bridge, 1989, Relaxation of rabbit ventricular muscle by $\mathrm{Na}$-Ca exchange and sarcoplasmic reticulum calcium pump, Circ. Res., 65: 334-342.

Bevan J.A. and I. Laher, 1991, Pressure and flow-dependent vascular tone, Faseb. J., 5: 2267-2273.

Birnbaumer L., 1990a, G proteins in signal transduction, Ann. Rev. Pharmacol. Toxicol., 30: 675-705.

Birnbaumer L., 1990b, Transduction of receptor signal into modulation of effector activity by G-proteins: the first 20 years or so, Faseb. J., 4: 3068-3078.

Blackshear P.J., A.C. Nairin and J.F. Kuo, 1988, Protein kinases 1988: a current perspective, Faseb. 1., 2: 2957-2969.

Boels P.J., M. Troschka, J.C. Rüegg and G. Pfitzer, 1991, Higher Ca ${ }^{2+}$ sensitivity of tritonskinned guinea pig mesenteric microarteries as compared with large arteries, Circ. Res., 69: 989-996.

Bohlen H.G., 1986, Localization of vascular resistance changes during hypertension, tension., 8 : 181-183.

Bohlen H.G. and K. Aukland, 1991, Pressure profile in resistance arteries, in: Resistance Arteries. Structure and Function, Mulvany M.J., C.A. Aalkjaer, A.M. Heagerty, N.C.B. Nyborg and S. Strandgaard, Exerpta Medica, Amsterdam, The Netherlands, 24-25.

Bolton T.B., 1979, Mechanisms of action of transmitters and other substances on smooth muscle, Physiol. Rev., 3: 606-718.

Bolton T.B., S.P. Lim, D.M.W. Salmon and D.J. Beech, 1988, Calcium channels and calciummediated effects in smooth muscle cells, J. Cardiovasc. Pharmacol., 12: S96-S99.

Bolzon B.J. and D.W. Cheung, 1989, Isolation and characterization of single vascular smooth muscle cells from spontaneously hypertensive rats, Hypertension., 14: 137-144.

Boonen H.C.M. and J.G.R. De Mey, 1990a, G-proteins are involved in contractile responses of isolated mesenteric resistance arteries to agonists, Naunyn. Schmiedeberg's. Arch. Pharmacol., 342: 462-468.

Boonen H.C.M. and J.G.R. De Mey, 1990b, Increased calcium sensitivity in isolated resistance arteries from spontaneously hypertensive rats: effects of dihydropyridines, Eur. J. Pharmacol., 179: 403-412.

Boonen H.C.M. and J.G.R. De Mey, 1991, Effects of a phorbol ester and staurosporine on electro- and pharmacomechanical coupling in a resistance artery, Eur. J. Pharmacol., 202: 25-32. 


\section{REFERENCES}

Boonen H.C.M., P.M.H. Schiliers, G.E. Fazzi, G.M.J. Janssen, M.J.A.P. Daemen and J.G.R. De Mey, 1491, DNA synthesis in isolated arteries. Kinetics and structural consequences, Am. J. Physiol., 26(): H210-H217.

Borders L. and H.J. Granger, 1986, Power dissipation as a measure of peripheral resistance in vascular networks, Hypertension., 8: 184-191.

Boscoboinik D.O. C.E. Hensey and A. Azzi, 1990, Effect of staurosporine on the phorbol esterinduced activation of the $\mathrm{Na} / \mathrm{H}^{*}$ antiporter in smooth muscle cells, Biochim. Biophys. Acta., 1052: 499-502.

Boyer J.L., J.R. Hepler and T.K. Harden, 1989, Hormone and growth factor receptor-mediated regulation of phospholipase-C activity, TIPS, 10: 360-364

Boynton A.L., N.M. Dean and T.D. Hill, 1990, Inositol 1,3,4,5,-tetrakisphosphate and regulation of intracellular calcium, Biochem. Pharmacol., 40: 1933-1939.

Brown A.M. and L. Birnbaumer, 1988, Direct G-protein gating of ion channels, Am. J. Physiol., 254: $\mathrm{H} 401-\mathrm{H} 410$.

Brown A.M., A. Yatani, Y. lmoto, J. Codina, R. Mattera and L. Birnbaumer, 1989, Direct G-protein regulation of $\mathrm{Ca}^{2+}$ channels, Ann. N.Y. Acad. Sci., 560: 373-386.

Buchholz R.A., L. Dundore, W.R. Cuminsky, A.L. Harris and P.J. Silver, 1991, Protein kinase inhibitors and blood pressure control in spontaneously hypertensive rats, Hypertension., 17: 91-100.

Bukoski R.D., 1990, Intracellular $\mathrm{Ca}^{2+}$ metabolism of isolated resistance arteries and cultured vascular myocytes of spontaneously hypertensive and Wistar Kyoto normotensive rats, J. Hypertension., 8: 37-43.

Burnstock G., 1990, Local mechanisms of blood flow control by perivascular nerves and endothelium, ]. Hypertension., 8: S95-S106.

Burrows M.E. and P.C. Johnson, 1981, Diameter, wall tension, and flow in mesenteric arterioles during autoregulation, Am. J. Physiol., 241: H829-H837.

Carafoli E., 1987, Intracellular calcium homeostasis, Ann. Rev. Biochem., 56: 345-433.

Carrow R. and M.L. Calhoun, 1964, The extent of cardiac muscle in the great veins of the dog, Anat. Rec., 150: 249-256.

Casey P.J. and A.G. Gilman, 1988, G-Protein involvement in receptor-effector coupling, J. Biol. Chem., 263: 2577-2580.

Cauvin C. and C. Van Breemen, 1985, Effects of $\mathrm{Ca}^{2+}$ antagonists on isolated rabbit mesenteric resistance vessels as compared to rabbit aorta, in: Cardiovascular Effects of Dihydropyridine-type Calcium Antagonists and Agonists, Fleckenstein A., C. Van Breemen, R. Gross and F. Hoffmeister, Springer Verlag, Berlin, 259-269.

Cauvin C., R. Loutzenhiser and C. Van Breemen, 1983, Mechanisms of calcium antagonistinduced vasodilatation, Ann. Rev. Pharmacol. Toxicol., 23: 373-396.

Cauvin C., S. Lukeman, J. Cameron, O. Hwang and C. Van Breemen, 1985, Differences in norepinephrine activation and diltiazem inhibition of calcium channels in isolated rabbit aorta and mesenteric resistance arteries, Circ. Res, 56: 822-828.

Cauvin C., C. Gelband and C. Van Breemen, 1987, ${ }^{45} \mathrm{Ca}$ Fluxes in rat mesenteric resistance vessels, J. Cardiovasc. Pharmacol., 10: S44-S48.

Cauvin C., S.W. Weir, A. Wallnofer and U. Rüegg, 1988, Agonist-induced activation of rat mesenteric resistance vessels: comparison between noradrenaline and vasopressin, ]. Cardiovasc. Pharmacol., 12: S128-S133.

Chamley-Campbell J.H., R. Campbell and R. Ross, 1979, The smooth muscle cell in culture, Am. Physiol. Society., 59: 1-59.

Chamley-Campbell J.H. and G.R. Campbell, 1981, What controls smooth muscle phenotype?, Atherosclerosis, 40: 347-357.

Chamley-Campbell J.H., G.R. Campbell and R. Ross, 1981, Phenotype-dependent response of cultured aortic smooth muscle to serum milogens, J. Cell. Biol., 89: 379-383.

de Champlain J., 1990, Pre- and postsynaptic adrenergic dysfunctions in hypertension, J. Hypertension., 8: \$77-\$85. 
Chiu A.T., P.C. Wong and P.B.M.W.M. Timmermans, 1987, $\alpha_{1}$-Adrenoceptor induced $\mathrm{Ca}^{2 *}$ movements in rat aorta: antagonism by phenoxybenzamine and $\mathrm{N}$-ethoxycarbonyl-2ethoxy-1,2-dihydroquinoline, J. Pharmacol. Exp. Therap., 243: 577-583.

Christensen K.L., L.T. Jespersen and M.J. Mulvany, 1989, Development of blood pressure in spontaneously hypertensive rats after withdrawal of long-term treatment related to vascular structure, J. Hypertension., 7: 83-90.

Cook N.S., 1988, The pharmacology of potassium channels and their therapeutic potential, TIPS, 9: 21-28.

Cook N.S., S.W. Weir and M.C. Danzeisen, 1988, Anti-vasoconstrictor effects of $\mathrm{K}^{+}$-channel opener cromakalim on the rabbit aorta; comparison with the calcium antagonist isradipine, Br. J. Pharmacol., 95: 741-752.

Cox R.H., 1987, Contribution of salt to arterial wall changes in DOCA-hypertension in the rat, 1. Hypertension., 5: 611-619.

Daemen M.J.A.P., D.M. Lombardi, F.T. Bosman and S.M. Schwartz, 1991, Angiotensin II induces smooth muscle cell proliferation in the normal and injured rat arterial wall, Circ. Res., 68: 450-456.

Dainty I.A., J.C. McGrath, M. Spedding and A.G.B. Templeton, 1990, The influence of the initial stretch and the agonist-induced tone on the effect of basal and stimulated release of EDRF, Br. J. Pharmacol., 100: 767-773.

Daly C.J., J.C. McGrath and V.G. Wilson, 1988, An examination of the postjunctional $\alpha$-adrenoceptor subtypes for (-)-noradrenaline in several isolated blood vessels from the rabbil, Br. J. Pharmacol., 95: 473-484.

Daly C.J., W.R. Dunn, J.C. McGrath, D.J. Miller and V.G. Wilson, 1990, An examination of the sources of calcium for contractions mediated by postjunctional $\alpha_{1}$ - and $\alpha_{2}$-adrenoceptors in several blood vessels isolated from the rabbit, Br. J. Pharmacol., 99: 253-260.

De Mey J.C.R and D.L. Brutsaert, 1984, Mechanical properties of resting and active isolated coronary arteries, Circ. Res., 55: 1-9.

De Mey J.G.R. and S.D. Gray, 1985, Endothelium-dependent reactivity in resistance vessels, Prog. Appl. Microcirc, 8: 181-187.

De Mey J.G.R. and P.M. Vanhoutte, 1981, Uneven distribution of postjunctional $\alpha_{1}$ - and $\alpha_{2}$ like adrenoceptors in canine arterial and venous smooth muscle, Circ. Res., 48: 875-884.

De Mey J.G.R. and P.M. Vanhoutte, 1982, $\mathrm{Na}^{+}-\mathrm{K}^{+}$exchanges in canine arterial and venous smooth muscle, Am. J. Physiol., 243: H551-H559.

De Mey J.G.R., G. Burnstock and P.M. Vanhoutte, 1979, Modulation of the evoked release of noradrenaline in canine saphenous vein via presynaptic receptors for adenosine but not ATP, Eur. J. Pharmacol, 55: 401-405.

De Mey J.G.R., A. Lenaers, G. Defreyn, P. Calderon and J. Roba, 1987, Arterial reactivity, blood pressure, and plasma levels of atrial natriuretic peptides in normotensive and hypertensive rats: effects of acute and chronic administration of atriopeptin III, J. Cardiovasc. Pharmacol., 9: 525-535.

De Mey J.G.R., H.C.M. Boonen and H.A.J. Struijker Boudier, 1988, Rhythmic contractile activity in resistance-sized arteries of spontaneously hypertensive rats, in: Resistance Arteries, Halpern W., B. Pegram, J.E. Brayden, M.K. McLaughlin and G. Osol, Perinatology Press, New York, 336-341.

De Mey I.G.R., M.P. Uitendaal, H.C.M. Boonen, M.J.J.F. Vrijdag, M.J.A.P. Daemen and H.A.J. Struijker Boudier, 1989, Acute and long term effects of tissue culture on contractile reactivity in renal arteries of the rat, Circ. Res., 65: 1125-1135.

De Mey J.G.R., M.J.A.P. Daemen, H.C.M. Boonen, F.T. Bosman, E.H. Dijkstra, G.E. Fazzi, G.M.J. Janssen, P.M.H. Schiffers, H.A.J. Strijker Boudier and M.J.J.F. Vrijdag, 1991a, In vivo DNA synthesis is not uniformely increased in arterial smooth muscle of young spontaneously hypertensive rats, J. Hypertension., 9: 695-701. 


\section{REFERENCES}

De Mey J.G.R., M.P. Uitendaal, H.C.M. Boonen, P.M.H. Schiffers and G.E. Fazzi, 1991b, Growth response's in isolated elastic, muscular and resistance-sized arterial segments of the rat, Blood. Vessels., 28: 372-385.

Dominiczak A.F. and D.F. Bohr, 1989, Vascular smooth muscle in hypertension, J. Hypertension., 7: S107-S115.

D'Orleans-Juste P., S. Dion, J. Mizrahi and D. Regoli, 1985, Effects of peptides and non-peptides on isolated arterial smooth muscles: role of the endothelium, Eur. J. Pharmacol., 114: 9-12.

Dostal D.E., J.A. Johnson, P.B. Langevin and N. Abas, 1988, Hyperresponsiveness of arterial rings induced by renal prehypertensive rabbit plasma, Am. J. Physiol., 255: H272-H279.

Drenth J.P.H., J. Nishimura, V.L.A. Nouailhetas and C. Van Breemen, 1989, Receptor-mediated $C$-kinase activation contributes to $\alpha$-adrenergic tone in rat mesenteric resistance artery, J. Hypertension., 7: S41-S45.

Droogmans G. and G. Callewaert, 1986, $\mathrm{Ca}^{+*}$-channel current and its modification by the dihydropyridine agonist Bay K8644 in isolated smooth muscle cells, Pflügers. Arch. Pharmacol., 406: 259-265.

Droogmans G., L. Raeymaekers and R. Casteels, 1977, Electro- and pharmaco-mechanical coupling in the smooth muscle cells of the rabbit ear artery, J. Gen. Physiol., 70: 129-148.

Duling B.R., T. Matsuki and S.S. Segal, 1991, Conduction in the resistance-vessel wall, in: The Resistance Vasculature, Bevan J.A., W. Halpern and M.J. Mulvany, Humana Press, Totowa, New Jersey, USA, 193-215.

Edwards R.M., 1985, Response of isolated renal arterioles to acetylcholine, dopamine and bradykinin, Am. J. Physiol., 248: F183-F189.

Eerdmans P.H.A., B.J. Heesen, H.A.J. Strujjker Boudier and J.G.R. De Mey, 1991, Sympathetic heterogeneity in mesenteric and renal resistance arteries, in: Resistance Arteries. Structure and Function, Mulvany M.J., C. Aalkjaer, A.M. Heagerty, N.C.B. Nyborg and S. Strandgaard, Excerpta Medica, Amsterdam, The Netherlands, 156-159.

Egleme C., F. Crussier and J.M. Wood, 1990, Local formation of angiotensin-II in the rat aorta effect of endothelium, Br. J. Pharmacol., 100: 237-240.

Eriksen S., K.L. Christensen, N.K. Thybo, N. Korsgaard and M.J. Mulvany, 1992, A negative correlation between perindopril dose and persistent effects on blood pressure in spontaneously hypertensive rats after withdrawal of treatment, Acta. Physiol. Scand., 146(S608): 74 (abstract).

Esler M., G. Lambert and G. Jennings, 1990, Increased regional sympathetic nervous activity in human hypertension: causes and consequences, J. Hypertension., 8: \$53-\$57.

Exton J.H., 1988, Mechanisms of action of calcium-mobilizing agonists: some variations on a young theme, Faseb. J., 2: 2670-2676.

Fabbro D., N. Mazureck, C. Borner, J.F. Conscience and P. Erne, 1988, Role of protein kinast-C (PKC) in short and long term cellular responses: inhibition of agonist-mediated calcium transients and down regulation of PKC, J. Cardiovasc. Pharmacol., 12: S7.3-S74.

Faber J.E., 1988, In situ analysis of $\alpha$-adrenoceptors on arteriolar and venular smuoth muscle in rat skeletal muscle microcirculation, Circ. Res., 62: 37-50.

Faber J.E. and G.A. Meininger, 1990, Selective interaction of $\alpha$-adrenoceptors with myogenic regulation of microvascular smooth muscle, Am. J. Physiol., 259: H1126-H1130.

Fish R.D., G. Sperti, W.S. Colucci and D.E. Clapham, 1988, Phorbol ester increases the dihydropyridine-sensitive calcium conductance in a vascular smooth muscle cell line, Circ. Res., 62: 1049-1054.

Fleckenstein A., 1964, Die Bedeutung der Energiereichen Phosphate für Kontraktilität und Tonus des Myokards, Verh. Dtsch. Ges. Inn. Med., 70: 81-99.

Fleckenstein A., (eds.), 1983, Calcium Antagonism in Heart and Smooth Muscle, John Wiley \& Sons, New York, USA, 400. 
Fleckenstein A., C. Van Breemen, R. Gross and F. Hoffmeister, (eds.), 1984, Cardinvascular effects of dihydropyridine-type antagonists and agonists, Springer Verlag, Berlin, Germany, 511.

Fleckenstein A., G. Fleckenstein-Grü, M. Frey and J. Zorn, 1987, Future directions in the use of calcium antagonists, Am. J. Cardiol., 59: 177B-187B.

Folkow B., 1978, Cardiovascular structural adaptation; its role in the initiation and maintenance of primary hypertension, clin, 55: 3-22.

Folkow B., 1982, Physiological aspects of primary hypertension, Physiol. Rev., 62: 347-504.

Folkow B., 1986, The structural cardiovascular factor in hypertension- pressure dependence and genetic reinforcement, J. Hypertension., 4: S51-S56.

Folkow B., 1990, Structural factor in primary and secondary hypertension, Hypertension., 16: 89-101.

Folkow B., G. Grimby and O. Thulesius, 1958, Adaptive structural changes of the vascular walls in hypertension and their relation to the control of peripheral resistance, Acta. Physiol. Scand., 44: 255-272.

Forder J., A. Scriabine and H. Rasmussen, 1985, Plasma membrane calcium flux, protein kinase-C activation and smooth muscle contraction, J. Pharmacol. Exp. Therap., 235: 267-273.

Freslon J.L. and J.F. Guidicelli, 1985, Vascular effects of captopril and dihydralazine in spontaneously hypertensive rats. Prog. Appl. Microcirc., 8: 142-151.

Furchgott R.F., 1984, The role of endothelium in the responses of vascular smooth muscle to drugs, Ann. Rev. Pharmacol. Toxicol., 24: 175-197.

Furchgott R.F. and P.M. Vanhoutte, 1989, Endothelium-derived relaxing and contracting factors, Faseb. J., 3: 2007-2018.

Furchgott R.F. and J.V. Zawadski, 1980, The obligatory role of endothelial cells in the relaxation of arterial smooth muscle by acetylcholine, Nature., 288: 373-376.

Genest J., O. Kuchel, P. Hamet and M. Cantin, (eds.), 1983, Hypertension, McGraw-Hill Book Company, USA, 1318.

Gilbert E.K., B.A. Weaver and C.M. Rembold, 1991, Depolarization decreases the $\left[\mathrm{Ca}^{2+}\right]_{1}$ sensitivity of myosin light chain kinase in arterial smooth muscle: comparison of aequorin and Fura $2\left[\mathrm{Ca}^{2+}\right]$, estimates, Faseb. J., 5: 2593-2599.

Gill D.L., 1989, Calcium signalling : receptor kinships revealed, Nature., 342: 16-18.

Gilman A.G., 1987, G proteins: transducers of receptor-generated signals, Ann. Rev. Biochem., 56: 615-649.

Gleason M.M. and S.F. Flaim, 1986, Phorbol ester contracts rabbit thoracic aorta by increasing intracellular calcium and by activating calcium influx, Biochem. Biophys. Res. Commun., 138: $1362-1369$

Glossman H., J. Striessning, H.G. Knaus, J. Muller, A. Grassegger, H.D. Holtje, S. Marrer, L. Hymel and H.G. Schindler, 1989, Structure of calcium channels, Ann. N.Y. Acad. Sci., 560: 198-214.

Godfraind T. and A. Kaba, 1972, The role of calcium in the action of drugs on vascular smooth muscle, Arch. Int. Pharmacodyn. Ther., 196: 35-49.

Godfraind T., A. Kaba and P. Polster, 1968, Differences in sensitivity of arterial smooth muscles to inhibition of their contractile response to depolarization by potassium, Arch. Int Pharmacodyn. Ther., 172: 235-239.

Godfraind T., P.N.M. Vanhoutte, S. Govoni and R. Paoletti, (eds.), 1985, Calcium Entry Blockers and Tissue Protection, Raven Press, New York, USA, 261.

Gordon A.M., A.F. Huxley and F.J. Julian, 1966, The variation in isometric tension with sarcomere length in vertebrate muscle fibers, J. Physiol., 184: 170-192.

Gratzner H.G., 1982, Monoclonal antibody to 5-bromo- and 5-iododeoxyuridine: a new reagent for detection of DNA replication, Science., 128: 474-475.

Gray S.D., 1984, Spontaneous hypertension in the neonatal rat, Clin. Exp. Hypertension., A6: 755-781. 


\section{REFERENCES}

Graziano M.P. and A.G. Gilman, 1987, Guanine nucleotide-binding regulatoty proteins: mediators of transmembrane signalling, TIPS, 8: 478-481.

Griendling K.K., B.C. Berk, P. Ganz, M.A. Gimbrone and R.W. Alexander, 1987, Angiotensin II stimulation of vascular smooth muscle phosphoinositide metabolism, Hypertension., 9: IIII81-III185.

Griendling K.K., T. Tsuda, B.C. Berk and R.W. Alexander 1989 , Angiotensin 11 stimulation of vascular smooth muscle, J. Cardiovasc. Pharmacol., 4: S27-S33.

Griffin S.A., W.C.B. Brown, F. MacPherson, J.C. McGrath, V.C. Wilson, N. Korsgaard, M.J. Mulvany and A.F. Lever, 1991, Angiotensin II causes vascular hypertrophy in part by a non-pressor mechanism, Hypertension, 17: 626-635.

Griffith T.M. and D.H. Edwards, 1990, Myogenic autoregulation of flow may be inversely related to endothelium-derived relaxing factor activity, Am. J. Physiol., 258: H1171-H1180.

Grillone L.R., M.A. Clark, R.W. Godfrey, F. Stassen and S.T. Crooke, 1988, Vasopressin induces $V 1$ receptors to activate phosphatidylinositol and phosphatidylcholine-specific phospholipase- $C$ and stimulates the release of arachidonic acid by at least two pathways in the smooth muscle cell line, A-1, J. Biol. Chem., 263: 2658-2663.

Grynkiewicz G., M. Poenie and R.Y. Tsien, 1985, A new generation of Caiv indicators with greatly improved fluorescence properties, J. Biol. Chem., 260: 3440-3450.

Hai C.M. and R.A. Murphy, 1989, Ca*', crossbridge phosphorylation, and contraction, Ann. Rev. Physiol., 51: 285-298.

Han C., P.W. Abel and K.P. Minneman, 1987, $\alpha_{1}$-Adrenoceptor subtypes linked to different mechanisms for increasing intracellular $\mathrm{Ca}^{2+}$ in smooth muscle, Naturc., 329:333.

Harder D.R. and K. Hermsmeyer, 1983, Membrane mechanisms in arterial hypertension, Hypertension., 5: 404-408.

Harris D.E. and D.M. Warshaw, 1991, Length vs. active force relationship in single isolated smooth muscle cells, Am. J. Physiol., 260: C1104-C1112.

Hathaway D.R., K.L. March, ].A. Lash, L.P. Adam and R.L. Wilensky, 1989, Vascular smooth muscle. A review of the molecular basis of contractility. Circulation., 80: 219-233.

Head R.J., 1989, Hypernoradrenergic innervation: its relationship to functional and hyperplastic changes in the vasculature of the spontaneously hypertensive rat, Blood. Vessels., 26: 1-20.

Heesen B.J. and J.G.R. De Mey, 1990, Effects of cyclic AMP-affecting agents on contractile re activity of isolated mesenteric and renal resistance arteries of the rat, $\mathrm{Br}$. J. Pharmacol., 101: 859-864.

Hermsmeyer K., 1982, Electrogenic ion pumps and other determinants of membrane potential in vascular smooth muscle. The Physiologist, 25: 454-465.

Hermsmeyer K., 1983, Excitation of vascular muscle by norepinephrine, An. Biomed. Engin., 11: $567-577$.

Hermsmeyer K., 1987, Vascular muscle membrane cation mechanisms and total peripheral resistance, Hypertension., 10: I10-[22.

Hermsmeyer K. and D. Harder, 1986, membrane ATP-ase mechanisms of $K^{*}$-return relaxation in arterial muscles of stroke-prone SHR and WKY, Am. J. Physiol., 250: C557-C 562.

Hermsmeyer K. and N.J. Rusch, 1989, Calcium channel alterations in genetic hypertension, Hypertension, 14: 453-456.

Hermsmeyer K., M. Sturek, W. Marvin, R. Mason and A. Puga, 1989, Vascular muscle calcium channel modulation in hypertension, J. Cardiovasc. Pharmacol., 14: S45-S48.

Hess P., J.B. Lansman and R.W. Tsien, 1984, Different modes of calcium channel gating behavior favoured by dihydropyridine calcium agonists and antagonists, Nature., 311: 538-544.

Hill M.A., J.C. Falcone and G.A. Meininger, 1990, Evidence for protein kinase-C involvement in arteriolar reactivity, Am. J. Physiol,, 259: H1586-H1594.

Himpens B., T. Kitazawa and A.P. Somlyo, 1990, Agonist-dependent modulation of $\mathrm{Ca}^{2}$. sensitivity in rabbit pulmonary artery smooth muscle, Pflügers Arch. Physiol., 417: 21-28. 


\section{REFERENCES}

Hirst G.D.S. and F.R. Edwards, 1989, Sympathetic neuroeffector transmission in arteries and arterioles, Physiol. Rev., 69: 546-604.

Ho A.K., T.P. Thomas, C.L. Chik, W.B. Anderson and D.C. Klein, 1988, Protein kinase C: subcellular redistribution by increased $\mathrm{Ca}^{2 *}$-influx, J. Biol. Chem., 263: 9292-9297.

Hogestätt E.D. and K.E. Andersson, 1984, Mechanisms behind the biphasic contractile response to potassium depolarization in isolated rat cerebral arteries, J. Pharmacol. Exp. Therap., 228: 187-195.

Hosey M.M. and M. Lazdunski, 1988, Calcium channels : molecular pharmacology, structure and regulation, J. Membr. Biol., 104: 81-105.

Huang Y.T., C.A. Hamilton and J.L. Reid, 1989, Endothelin stimulates phosphatidylinositol hydrolysis in rat vascular smooth muscles, J. Hypertension., 7: 703-705.

Huganir R.L. and P. Greengard, 1987, Regulation of receptor function by protein phosphorylation, TIPS, 8: 472-477.

Hurwitz L., 1986, Pharmacology of calcium channels and smooth muscle, Ann. Rev. Pharmacol. Toxicol, 26: 225-258.

Hutcheson I.R. and T.M. Griffith, 1991, Release of endothelium-derived relaxing factor is modulated by frequency and amplitude of pulsatile flow, Am. J. Physiol., 261: H257-H262.

Hwa J.I. and J.A. Bevan, 1986a, A nimodipine resistant $\mathrm{Ca}^{2+}$ pathway is involved in myogenic tone in resistance artery, Am. J. Physiol., 251: H182-H189.

Hwa J.J. and J.A. Bevan, 1986b, Stretch-dependent (myogenic) tone in rabbit ear resistance arteries, Am. J. Physiol, 250: H87-H95.

Jensen P.E., M.J. Mulvany and C. Aalkjaer, 1992, Endogenous and exogenous agonist induced changes in the coupling between $\left[\mathrm{Ca}^{2+}\right]_{i}$ and force in rat resistance arteries, Pflügers Arch. Physiol., 420: 536-543.

Jiang M.J, and K.G. Morgan, 1987, Intracellular calcium levels in phorbol ester-induced contractions of vascular muscle, Am. J. Physiol., 253: H1365-H1371.

Jimenez A.E. and I.G. Joshua, 1988, Predisposition to hypertension in rat pups from renal hypertensive rats, Clin. Exp. Hypertension., 10: 79-90.

Johansson B., 1987, Calcium and regulation of contraction: a short review, J. Cardiovasc. Pharmacol., 10: S9-S13.

Johansson B., 1989, Myogenic-tone and reactivity: definitions based on muscle physiology, J. Hypertension., 7: S5-S8.

Johnson M.D., H.Y. Wang, D. Ciechanowski and E. Friedman, 1991, Reduced G-protein function in desensitized rat aorta, J. Pharmacol. Exp. Therap., 259: 255-259.

Johnson P.C., 1980, The myogenic response, in: The Cardiovascular System. Vascular Smooth Muscle, Am Physiol. Soc., Bethesda, MD, 409-442.

Jones A.W., B.B. Geisbuhler, S.D. Shukla and J.M. Smith, 1988, Altered biochemical and functional responses in aorta from hypertensive rats, Hypertension., 11: 627-634.

Julou G. and J.L. Freslon, 1986, Effects of calcium entry blockers on $\mathrm{Ca}^{2+}$ induced contraction of depolarized and noradrenaline-exposed rat resistance vessels, Eur. J. Pharmacol., 129: $261-270$.

Julou-Schaeffer G. and J.L. Freslon, 1987, Compared effects of calcium entry blockers on calcium-induced tension in rat isolated cerebral and peripheral resistance vessels, Naunyn. Schmiedeberg's. Arch. Pharmacol, 336: 670-676.

Kamm K.E. and J.T. Stull, 1989, Regulation of smooth muscle contractile elements by second messengers, Ann. Rev. Physiol., 51: 299-313.

Karaki H., K. Sato and H. Ozaki, 1988. Different effects of norepinephrine and $\mathrm{KCl}$ on the cytosolic $\mathrm{Ca}^{2+}$ tension relationship in vascular smooth muscle of rat aorta, Eur. J. Pharmacol., 151: 325-328.

Kass G.E.N., J. Llopis, S.C. Chow, S.K. Duddy and S. Orrenius, 1990, Receptor-operated calcium influx in rat hepatocytes -identification and characterization using manganese, J. Biol. Chem., 265: 17486-17492. 


\section{REFERENCES}

Kato T., K. Iwama, H. Hashimoto, T. Ito and T. Satake, 1990, Prostaglandin H2 may be the endothelium-derived contracting factor released by acetylcholine in the aorta of the rat, $\mathrm{Hy}$ pertension., 15: 475-481

Kawasaki H., K. Takasaki, W.H. Cline and C. Su, 1988a, Effect of angiotensin III (des-aspl-angiotensin [1) on the vascular adrenergic neurotransmission in spontaneous]y hypertensive rats, Eur. J. Pharmacol., 147: 125-130.

Kilwasaki H., K. Takasaki, A. Saito and K. Goto, 1988b, Calcitonin gene-related peptide acts as a novel vasodilator neurotransmitter in mesenteric resistance vessels of the rat, Nature., 335: $164-16 \bar{j}$.

Khalil R.A., N. Lodge, K. Saida and C. Van Breemen, 1987, Mechanism of calcium activation in vascular smooth muscle, J. Hypertension., 5: S5-S15.

Khalil R.A. and C. Van Breemen, 1990, Intracellular free calcium concentration/force relationship in rabbit inferior vena cava activated by norepinephrine and high $\mathrm{K}^{*}$, Pflügers. Arch. Pharmacol., 416: 727-734.

Khalil R.A. and C. Van Breemen, 1988, Sustained contraction of vascular smooth muscle: calcium influx or C-kinase activation?, J. Pharmacol. Exp. Therap., 244: 537-542.

Kirber M.T., J.V. Walsh and J.J. Singer, 1988, Stretch-activated ion channels in smonth muscle: a mechanism for the initiation of stretch-induced contraction, Pflügers Arch. Physiol., 412: 3.39-345.

Kishi K. and T. Inoue, 1990, Possible mechanisms of abnormal norepinephrine sensitivity and reactivity of resistance vessels and the development of hypertension in spontaneously hypertensive rats. A hypothesis, Am. J. Hypertension., 3: 2025-205S.

Kitazawa T., B.D. Gaylinn, G.H. Denney and A.P. Somlyo, 1991, G-protein-mediated $\mathrm{Ca}^{2+}$ sensitization of smooth muscle contraction through myosin light chain phosphorylation, J. Biol. Chem, 266: 1708-1715.

Kocher O., O. Skalli, D. Cerutti, F. Gabbiani and G. Gabbiani, 1985, Cytoskeletal features of rat aurtic cells during development. An electron microscopic, immunohistochemical, and biochemical study, Circ. Res., 56: 829-838.

Kojima M., M. Asano, K. Aoki, M. Yamamoto and T. Matsuda, 1989, Decrease in $\alpha_{1}$-adrenoceptor reserve in mesenteric arteries isolated from spontaneously hypertensive rats, J. Hypertension., 7: 873-878.

Kumori S. and T.B. Bolton, 1989, Actions of guanine nucleotides and cyclic nucleotides on calcium stores in single patch-clamped smooth muscle cells from rabbit portal vein, $\mathrm{Br}$. J. Pharmacol., 97: 973-982.

Kondo K., O. Kozawa and K. Takatsuki, 1989, Ca ${ }^{2+}$-influx stimulated by vasopressin is mediated by phosphoinositide hydrolysis in rat smooth muscle cells, Biochem. Biophys. Res. Commun., 161: 677-682.

Kong J.Q., A. Taylor and W.W. Fleming, 1991, Mesenteric vascular responses of young spontaneously hypertensive rats, J. Pharmacol. Exp. Therap., 258: 13-17.

Korner P.I. and J.D. Swales, 1991, Debate on the role of resistance arteries in hypertension, in: Resistance Arteries, Mulvany M.J., C. Aalkjaer, A.M. Heagerty, N.C.B. Nyborg and S. Strandgaard, Excerpta Medica, Amsterdam, the Netherlands, 39-43.

Korner P.l., A. Bobik, J.A. ANgus, M.A. Adams and P. Friberg, 1989, Resistance control in hypertension, J. Hypertension., 7: S125-S134.

Korstanje C. and P.A. van Zwieten, 1987, Characterization of $\alpha$-adrenuceptor mediated reduction of flow in a saline-perfused constant pressure model of rat perfused hindquarters, J. Auton. Pharmacol., 7: 71-86.

Koutouzov S., A. Remmal, P. Marche and P. Meyer, 1987, Hypersensitivity of phospholipase-C in platelets of spontaneously hypertensive rats, Hypertension., 10: 497-504.

Laher I. and I.A. Bevan, 1987, Protein kinase-C activation selectively augments a stretch-induced, calcium-dependent tone in vascular smooth muscle, J. Pharmacol. Exp. Therap., 242: 566-572. 


\section{REFERENCES}

Laher I. and J.A. Bevan, 1989, Stretch of vascular smooth muscle activates tone and $\mathrm{Ca}^{2 *}$ influx, J. Hypertension., 7: S17-S20.

Laher I. and C. Triggle, 1983, The relationship between the elevated blood pressure of the spontaneously hypertensive rat and the chemical sensitivity of smooth muscle to adrenergic agents, Can. J. Physiol. Pharmacol., 62: 94-100.

Laher I. and C.R. Triggle, 1984, Pharmacological studies of smooth muscle from Dahl saltsensitive and salt-resistant rats, Can. J. Physiol. Pharmacol, 62: 101-104.

Laher I., P. Vorkapic, A.L. Dowd and J.A. Bevan, 1989, Protein kinase-C potentiates stretch induced cerebral artery tone by increasing intracellular sensitivity to $\mathrm{Ca}^{2 *}$, Biochem. Biophys. Res. Commun., 165: 312-318.

Lais L.T. and M.J. Brody, 1978, Vasoconstrictor hyperresponsiveness: an early pathogenic mechanism in the spontaneously hypertensive rat, Eur. J. Pharmacol., 47: 177-189.

Lamontagne D., U. Pohl and R. Busse, 1992, Mechanical deformation of vessel wall and shear stress determine the basal release of endothelium-derived relaxing factor in the intact rabbit coronary vascular bed, Circ. Res., 70: 123-130.

Langille B.L., M.P. Bendeck and F.W. Keeley, 1989, Adaptations of carotid arteries of young and mature rabbits to reduced carotid blood flow, Am. J. Physiol., 256: H931-H939.

Lash J.M., H.G. Bohlen and L. Waite, 1991, Mechanical characteristics and active tension generation in rat intestinal arterioles, Am. J. Physiol., 260: H1561-H1574.

Lee R.M.K.W., 1987, Structural alterations of blood vessels in hypertensive rats, Can. J. Pharmacol., 65: 1528-1535.

Lee R.M.K.W. and J.S. Smeda, 1985, Primary versus secondary structural changes of the blood vessels in hypertension, Can. J. Physiol. Pharmacol., 63: 392-401.

Lee R.M.K.W., J.B. Forrest, R.E. Garfield and E.E. Daniel, 1983, Comparison of blood vessel wall dimensions in normotensive and hypertensive rats by histometric and morphometric methods, Blood. Vessels., 20: 245-254.

Lever A.F., 1986, Slow pressor mechanisms in hypertension: a role for hypertrophy of resistance vessels?, J. Hypertension., 4: 515-524.

Liebau S., J. Hohlfeld and U. Forsterman, 1989. The inhibition of $\alpha_{1}$-adrenoceptor-mediated contractions of rabbit pulmonary artery by $\mathrm{Ca}^{2+}$-withdrawal, pertussis toxin and $\mathrm{N}$-ethylmaleimide is dependent on agonist intrinsic efficacy, Naunyn. Schmiedeberg's. Arch. Pharmacol., 339: 496-502.

Lincoln T.M., 1989, Cyclic GMP and mechanisms of vasodilatation, Pharmac. Ther., 41: 479-502.

Lindpaintner K. and D. Ganten, 1991, The cardiac renin-angiotensin system. An appraisal of present experimental and clinical evidence, Circ. Res., 68: 905-921.

Litten R.Z., E.A. Suba and B.L. Roth, 1987, Effects of a phorbol ester on rat aortic contraction and calcium influx in the presence and absence of Bay K 8644, Eur. J. Pharmacol., 144: 185.101.

Ljung, B., 1990, Vascular selectivity of felodipine: Experimental pharmacology, J. Cardiovasc. ['harmacol., 15: S11-S10.

Loirand G., P. lacaud, J. Mironneau and C. Mironneau, 1986, Evidence for two distinct calcium channels in rat vascular smooth muscle cells in short term primary culture, Pflügers. Arch. Pharmacol., 407: 566-568.

Lorimer A.R., D. Elmfeldt, G. Johnsson and A. Ward, 1985, First international meeting on felodipine, Drugs., 29: 1-212.

Luscher T.F., 1990a, The endothelium. Target and promoter of hypertension ?, Hypertension., 15: $482-485$.

Lüscher T.F., 1990b, Inbalance of endothelium-derived relaxing and contracting factors. A new concept in hypertension?, Am. J. Hypertension., 3: 317-330.

Lundberg J.M., A. Franco-Cereda, A. Hemsen, J.S. Lacroix and J. Pernow, 1990, Pharmacology of noradrenaline and neuropeptide tyrosine (NPY)-mediated sympathetic cotransmission, Fund. Clin. Pharmacol., 4: 373-391. 


\section{REFERENCES}

Lüscher T.F. and P.M. Vanhoutte, 1988, Endothelium-dependent responses in human blond vessels, TIPS, 9: 181-184.

Mark A.L., 1991, Sympathetic neural contribution to Salt-induced hypertension in Dahl rats, Hypertension., 17: I86-190.

McGillivray-Anderson K.M. and J.E. Faber, 1990, Effect of acidosis on contraction of micro vascular smooth muscle by $\alpha_{1}$ - and $\alpha_{2}$-adrenoceptors. Implications for neural and metabolic regulation, Circ. Res., 66: 1643-1657.

McMahon E.G. and R.J. Paul, 1985, Calcium sensitivity of isometric force in intact and chemically skinned aortas during the development of aldosterone-salt hypertension in the rat, Circ. Res., 56: 427-435.

Meininger G.A. and J.E. Faber, 1991, Adrenergic facilitation of myogenic response in skeletal muscle arterioles, Am. J. Physiol., 260: H1424-H1432.

Meininger G.A., M.J. Davis, J.C. Falcone, M.A. Hill and D.C. Zawieja, 1991a, Mechanisms of the myogenic response in blood flow autoregulation, in: Resistance Arteries, Mulvany M.J., C. Aalkjaer, A.M. Heagerty, N.C.B. Nyborg and S. Strandgaard, Excerpta Medica, Amsterdam, the Netherlands, 173-177.

Meininger G.A., J.C. Falcone and M.A. Hill, 1991b, Autoregulation and resistance-artery function, in: The Resistance Vasculature, Bevan J.A., W. Halpern and M.J. Mulvany, Humana, Press, Totowa, New Jersey, USA, 345-371.

Meldolesi J. and T. Pozzan, 1987, Pathways of $\mathrm{Ca}^{2+}$-influx at the plasma membrane : voltagereceptor- and second messenger-operated channels, Exp. Cell. Res., 171: 271-283.

Mellander S., 1989, Functional aspects of myogenic vascular control, J. Hypertension., 7: S21-S30.

Messing M.W.J., H. Van Essen, T.L. Smith, J.F.M. Smits and H.A.J. Struijker Boudier, 1991, Microvascular actions of calcium channel antagonists, Eur. J. Pharmacol., 198: 189-195.

Messing M.W.J., H. van Essen and H.A.J. Struijker Boudier, 1990, Effects of $\alpha$-adrenoceptor antagonists on striated muscle microcirculation of conscious rats, Drugs., 40: 31-33.

Michel M.C., R. Kanczik, M. Khamssi, A. Knor, H. Siegl, J.J. Beckeringh and O.E. Brodde, 1989, $\alpha$ - And $\beta$-adrenoceptors in hypertension. 1. Cardiac and renal $\alpha_{1}-\beta_{1}$ - and $\beta_{2}$-adrenoceptors in rat models of acquired hypertension, J. Cardiovasc. Pharmacol., 13: 421-431.

Michel M.C., O.E. Brodde and P.A. Insel, 1990a, Peripheral adrenergic receptors in hypertension, Hypertension., 16: 107-120.

Michel M.C., G. Hanft and G. Gross, 1990b, $\alpha_{11}$ but not $\alpha_{1 \mathrm{~A}}$ adrenoceptors mediate inositol phosphate generation, Naunyn. Schmiedeberg's. Arch. Pharmacol,, 341: 385-387.

Michel M.C., S. Jager, R. Casto, R. Rettig, C. Graf, M. Printz, P.A. Insel, T. Philipp and O.E. Brodde, 1992, On the role of renal $\alpha$-adrenergic receptors in spontaneously hypertensive rats, Hypertension., 19: 365-370.

Miller V.M., 1991, Interaction between neural and endothelial mechanisms in control of vascular tone, NIPS, 6: 60-63.

Miller V.M. and P.M. Vanhoutte, 1988, Enhanced release of endothelium-derived factor(s) by chronic increases in blood flow. Am. J. Med., 255: h446-h451.

Minneman K.P., 1988, $\alpha_{1}$-Adrenergic receptor subtypes, inositol phosphates and sources of cell calcium, Pharmacol. Rev., 40: 87-119.

Mitsuhashi T., R. Curtis-Morris and H.E. Ives, 1989, Endothelin induced increases in vascular smooth muscle $\mathrm{Ca}^{2 \cdot}$ do not depend on dihydropyridine-sensitive $\mathrm{Ca}^{2+}$ channels, J. Clin. Invest., $84: 635-639$.

Moncada S., R.M.J. Palmer and E.A. Higgs, 1989, Biosynthesis of nitric oxide from L-arginine. A pathway for the regulation of cell function and communication, Biochem. Pharmacol., 38: $1709-1715$.

Moreland R.S., S. Moreland and R.A. Murphy, 1988, Dependence of stress on length, Ca ${ }^{2 *}$, and myosin phosphorylation in skinned smooth muscle, Am. J. Physiol., 255: C473-c478. 


\section{REFERENCES}

Mori T, T. Yanagisawa and N. Taira, 1990, Phorbol 12,13-dibutyrate increases vascular tone but has a dual ation on intracellular calcium levels in porcine coronary arteries, Naunyn. Schmiedeberg's. Arch. Pharmacol., 341: 251-255.

Morrison K.J. and D. Pollock, 1990, Impairment of relaxations to acetylecholine and nitric oxide by a phorbol ester in rat isolated aorta, Br. J. Pharmacol, 101: 432-436.

Morton J.J., E.C. Beattie, S.A. Griffin, F. MacPherson, F. Lyel and D. Russo, 1990, Vascular hypertrophy, renin and blood pressure in the young spontaneously hypertensive rat, Clin. Sci., 79: 523-530.

Mulvany M.J., 1983, Do resistance vessel abnormalities contribute to the elevated blood pressure of spontaneously-hypertensive rats?, Blood. Vessels., 20: 1-22.

Mulvany M.J., 1986, Role of vascular structure in blood pressure development of the SHR, J. Hypertension., 4: S61-S63.

Mulvany M.J., 1987. The structure of the resistance vasculature in essential hypertension, J. Hypertension., 5: 129-136.

Mulvany M.J., 1988, Resistance vessel structure and function in the etiology of hypertension studied in F2-generation hypertensive-normotensive rats, J. Hypertension,, 6: 655-663.

Mulvany M.J., 1989, Structure and function of peripheral vascular smooth muscle in hypertension, J. Cardiovasc. Pharmacol., 14: S85-S89.

Mulvany M.J., 1990, Structure and function of small arteries in hypertension, J. Hypertension., 8: S225-S232.

Mulvany M.J. and C. Aalkjaer, 1990, Structure and function of small arteries, Physiol. Rev., 70 : 921-961.

Mulvany M.J. and W. Halpern, 1977, Contractile properties of small arterial resistance vessels in spontaneously hypertensive and normotensive rats, Circ. Res., 41: 19-26.

Mulvany M.J. and N. Nyborg, 1980, An increased calcium sensitivity of mesenteric resistance vessels in young and adult spontaneously hypertensive rats, $\mathrm{Br}$. J. Pharmacol., 71: 585-596.

Mulvany M.J. and D.M. Warshaw, 1979, The active tension-length curve of vascular smooth muscle related to its cellular components, J. Gen. Physiol., 74: 85-104.

Mulvany M.J., P.K. Hansen and C. Aalkjaer, 1978, Direct evidence that the greater contractility of resistance vessels in spontaneously hypertensive rats is associated with a narrowed lumen, a thickened media, and an increased number of smooth muscle cell layers, Circ. Res., 43: 854-864.

Mulvany M.J., H. Nilsson and J.A. Flatman, 1982, Role of membrane potential in the response of rat small mesenteric arteries to exogenous noradrenaline stimulation, J. Physiol., 332: 363-373.

Muramatsu I., T. Ohmura, T. Kigoshi, S. Hashimoto and M. Oshita, 1990, Pharmacological subclassification of $\alpha_{1}$-adrenoceptors in vascular smooth muscle, Br. J. Pharmacol., 99: 197-201.

Murayama T. and M. Ui, 1983, Loss of the inhibitory function of the guanine nucleotide regulatory component of adenylate cyclase due to its ADP ribosylation by islet activating factor, pertussis toxin, in adipocyte membranes, J. Biol. Chem., 258: 3319-3326.

Murphy R.A., 1978, Control of the actin-myosin interaction by $\mathrm{Ca}^{2+}$ in vascular smooth muscle, in: Mechanisms of Vasodilatation, Vanhoutte P.M. and I. Leusen, Karger, Basel, Switzerland, 30-36.

Neild T.O. and K. Keef, 1985, Measurements of the membrane potential of arterial smooth muscle in anesthetized animals and its relationship to changes in artery diameter, microvasc. Res., 30: 19-28.

Nelson M.T., N.B. Standen, J.E. Brayden and J.F. Worley III, 1988, Noradrenaline contracts arteries by activating voltage-dependent calcium channels, Nature., 336: 382-385.

Nelson M.T., J.B. Patlak, J.F. Worley III and N.B. Standen, 1990, Calcium channels, potassium channels, and voltage dependence of arterial smooth muscle tone, Am. J. Physiol,, 259: C3-C18. 


\section{REFERENCES}

Nichols A.J., E.D. Motley and R.R. Ruffolo, 1989. Effect of pertussis toxin treatment on post junctional $\alpha_{1}$-and $\alpha_{2}$-adrenoceptor function in the cardiovascular system of the pithed rat, J. Pharmacol. Exp. Therap., 249: 203-209.

Nielsen H, F.V. Mortensen, H.K. Pilegaard, J.M. Hasenkam and M.J. Mulvany, 1992, Calcium utilization coupled to stimulation of postjunctional $\alpha_{1}$ - and $\alpha_{2}$-adrenoceptors in isolated human resistance arteries, J. Pharmacol. Exp. therap., 260: 637-643.

Nilsson H. and N. Sjöblom, 1985, Distension-dependent changes in noradrenaline sensitivity in small arteries from the rat, Acta. Physiol. Scand., 125: 429-435.

Nishimura J., M. Kolber and C. Van Breemen, 1988, Norepinephrine and GTP- $\tau-S$ increase myofilament $\mathrm{Ca}^{2+}$ sensitivity in $\alpha$-toxin permeabilized arterial smooth muscle, Biochem. Biophys. Res. Commun., 157: 677-683.

Nishimura J., R.A. Khalil, J.P. Drenth and C. Van Breemen, 1990, Evidence for increased myo filament $\mathrm{Ca}^{2+}$ sensitivity in norepinephrine-activated vascular smooth muscle, Am. J. Physiol., 259: $\mathrm{H} 2-\mathrm{H} 8$.

Nishizuka Y., 1986, Studies and perspectives of protein kinase-C, Science., 233: 305-313.

Nordlander M.I.L., 1989, Inhibition of vascular myogenic tone and reactivity by calcium antagonists, J. Hypertension., 7: S141-S145.

Nyborg N.C.B. and M.]. Mulvany, 1984, Effect of felodipine, a new dihydropyridine vasodilator, on contractile responses to potassium, noradrenaline, and calcium in mesenteric resistance vessels of the rat, J. Cardiovasc. Pharmacol., 6: 499-505.

Ohanian J. and A.M. Heagerty, 1991, Diacylglycerol metabolism in vascular smooth muscle, in: Resistance Arteries, Mulvany M.J., C. Aalkjaer, A.M. Heagerty, N.C.B. Nyborg and S. Strandgaard, Excerpta Medica, Amsterdam, the Netherlands, 69-73.

Ohlstein E.H., S. Horohonich and D.W.P. Hay, 1989, Cellular mechanisms of endothelin in rabbit aorta, J. Pharmacol. Exp. Therap., 250: 548-555.

Ollerenshaw J.D., B. Lassegue, R.W. Alexander and K.K. Griendling, 1991, Intracellular signalling in arteries and vascular smooth muscle cells in culture, in: Resistance Arteries, Mulvany M.J., C. Alkjaer, A.M. Heagerty, N.C.B. Nyborg and S. Strandgaard, Excerpta Medica, Amsterdam, the Netherlands, 73-76.

Oriowo M.A. and R.R. Ruffolo, 1992, Heterogeneity of postjunctional $\alpha_{1}$-adrenoceptors in mammatian aortae: subclassification based on chloroethylclonidine, WB 4101 and nifedipine, J. Vasc. Res, 29: 33-40.

Osol G., I. Laher and M. Cipolla, 1991, Protein kinase-C modulates basal myogenic tone in resistance arteries from the cerebral circulation, Circ. Res., 68: 359-367.

Overbeck H.W., 1980, Pressure-independent increases in vascular resistance in hypertension: role of sympathoadrenergic influences, Hypertension., 2: 780-786.

Owens G.K., 1989, Control of hypertrophic versus hyperplastic growth of vascular smooth muscle cells, Am. J. Physiol., 257: H1755-H1765.

Palmer R.M.J., D.D. Rees, D.S. Ashton and S. Moncada, 1988, L-arginine is the physiological precursor for the formation of nitric oxide in endothelium-dependent relaxation, Biochem. Biophys. Res. Commun., 153: 1251-1256.

Plunkett W.C. and H.W. Overbeck, 1985, Increased arteriolar wall-to-lumen ratio in a normotensive vascular bed in coarctation hypertension, Am. J. Physiol., 249: H859-H866.

Plunkett W.C. and H.W. Overbeck, 1988, Arteriolar wall thickening in hypertensive rats urrelated to pressure or sympathoadrenergic influences, Circ. Res., 63: 937-943.

Price J.M., D.L. Davis and E.B. Knauss, 1981, Length-dependent sensitivity in vascular smooth muscle, Am. J. Physiol., 241: H557-H563.

Prieto D, M.J. Mulvany, N.C.B. Nyborg, 1991: (+)-S-12967 and (-)-S-12968: 1,4-dihydropyridine stereoisomers with calcium channel agonistic and antagonistic properties in rat resistance arteries, Br. J. Pharmacol., 103: 1703-1708.

Putney J.W., 1987, Calcium-mobilizing receptors, TIPS, 8: 481-486.

Rampe D. and D.J. Triggle, 1989, New advances in molecular pharmacology of $\mathrm{Ca}^{2+}$ channels, TIPS, 10: 388-389. 


\section{REFERENCES}

Rasmussen H., Y. Takuwa and S. Park, 1987, Protein kinase-C in the regulation of smooth muscle contraction, Faseb. J., 1: 177-185.

Raymond J.R., M. Hnatowich, R.J. Lefkowitz and M.G. Caron, 1990, Adrenergic receptors: Models for regulation of signal transduction processes, Hypertension., 15: 119-131.

Rembold C., 1991, Relaxation, $\left[\mathrm{Ca}^{2+}\right]_{1}$, and the latch-bridge hypothesis in swine arterial smooth muscle, Am. J. Physiol., 261: C41-C50.

Rembold C.M. and R.A. Murphy, 1990, Muscle length, shortening, myoplasmic $\left[\mathrm{Ca}^{2+}\right]$, and activation of arterial smooth muscle, Circ. Res., 66: 1354-1361.

Remmal A., S. Koutouzov, A. Girard, P. Meyer and P. Marche, 1988, Defective phosphoinositide metabolism in primary hypertension, Experientia., 44: 133-137.

Resink T.J., T. Scott-Burden, U. Baur, M. Burgin and F.R. Bühler, 1989, Enhanced responsiveness to angiotensin II in vascular smooth muscle cells from spontaneously hypertensive rats is not associated with alterations in protein kinase-C, Hypertension., 14: 293-303.

Ribeiro-Neto F.A. and M. Rodbell, 1989, Pertussis toxin induces structural changes in $\mathrm{G}_{r i}$-proteins independently of ADP-ribosylation, Proc. Natl. Acad. Sci. USA., 86: 2577-2581.

Richert E.A., P.J.F. Cleland, S. Rattigan and M.G. Clark, 1987, Contraction-associated translocation of protein kinase-C in rat skeletal muscle, Febs., 217: 232-236.

Rinaldi G. and D.F. Bohr, 1989, Potassium-induced relaxation of arteries in hypertension: modulation by extracellular calcium, Am. J. Physiol., 256: H707-H712.

Rosenthal W., J. Hescheler, W. Trautwein and G. Schultz, 1988, Controle of voltage-dependent $\mathrm{Ca}^{2+}$ channels by G-protein-coupled receptors, Faseb. J., 2: 2784-2790.

Rubanyi G.M., A.D. Freay, K. Kauser, A. Johns and D.R. Harder, 1990, Mechanoreception by the endothelium: mediators and mechanisms of pressure- and flow-induced vascular responses, Blood. Vessels., 27: 246-257.

Rubbia L. and G. Gabbiani, 1989, The cytoskeleton of arterial smooth muscle cells during development, atheromatosis, and tissue culture, J. Cardiovasc. Pharmacol., 14: 9-11.

Rügg U.T. and G.M. Burgess, 1989, Staurosporine, K-252 and UCN-01: potent but nonspecific inhibitors of protein kinases, TIPS, 10: 218-219.

Rüegg U.T., A. Wallnofer, S. Weir and C. Cauvin, 1989, Receptor-operated calcium-permeable channels in vascular smooth muscle, J. Cardiovasc. Pharmacol., 14: S49-S58.

Rump.LC, G. Ruff, V. Wolk and P. Schollmeyer, 1991, $\alpha_{2}$-Adrenoceptor activation inhibits noradrenaline release in human and rabbit isolated renal artery, Eur. J. Pharmacol., 196: 277-283.

Rusch N.]. and K. Hermsmeyer, 1988, Calcium currents are altered in the vascular muscle cell membrane of spontaneously hypertensive rats, Circ. Res., 63: 997-1002.

Sahlgren B., A.C. Eklof and A. Aperia, 1986, Studies of the renal component of the hypertension in rats with aortic constriction. Role of angiotensin II, Acta. Physiol. Scand., 127: 443-448.

Saida K. and C. Van Breemen, 1984, Characteristics of the norepinephrine sensitive $\mathrm{Ca}^{2+}$ store in vascular smooth muscle, Blood. Vessels., 21: 43-52.

Sasaguri T. and S.P. Watson, 1989, Protein-kinase-C regulates the tonic but not the phasic component of contraction in guinea-pig ileum, Br. J. Pharmacol., 98: 791-798.

Sasaki T, and D.V. (illacher, 1990, Extracellular ATP activates receptor-operated cation channels in mouse lacrimal acinar cells to promote calcium influx in the absence of phosphoinositide metabolism, Febs., 264: 130-134.

Schelling I'., H. Fincher and D. Ganten, 1991, Angiotensin and cell growth: a link to cardio vascular hypertrophy?, J. Hypertension., 9: 3-15.

Schmid. Schönhcin G.W., B.W. Zweifach, F.A. Delano and P.C.Y. Chen, 1987, Microvascular tone in a skeletal muscle of SHR, Hypertension., 9: 164-171.

Schwartz A., 1989, Calcium antagonists: review and perspective on mechanisms of action, Am. J. Cardiol., 64: 31-9]. 


\section{REFERENCES}

Schwartz S.M., G.R. Campbell and J.H. Campbell, 1986, Replication of smooth muscle cells in vascular disease, Circ. Res., 58: 427-444.

Scott-Burden T., T.J. Resink and F.R. Bühler, 1989, Enhanced growth and growth responsiveness of vascular smooth muscle cells from hypertensive rats, J. Cardiovase. ['harmacol., 14: S16-S21.

Scott-Burden T., T.J. Resink, A.W.A. Hahn and F.R. Bühler, 1992, Vasoactive peptides and growth factors in the pathophysiology of hypertension, J. Cardiovasc. Pharmacol., in press:

Scriabine A. and W. Van den Kerckhoff, 1988, Phanmacology of nimodipine. A review, Ann. N.Y. Acad. Sci., 522: 698-706.

Segal S.S., 1991, Microvascular recruitment in hamster striated muscle: role of conducted vaso dilation, Am. J. Physiol., 261: H181-H189.

Sharma R.V. and R.C. Bhalla, 1988, Calcium and abnormal reactivity of vascular smooth muscle in hypertension, Cell. Calcium., 9: 267-274.

Silver P.J., K. Walus and J. Disalvo, 1984, Adenosine-mediated relaxation and activation of cyclic-AMP-dependent protein kinase in coronary arterial smooth muscle, J. Pharmacol. Exp. Therap., 228: 342-347.

Smeda J.S. and E.E. Daniel, 1988, Elevations in arterial pressure induce the formation of spontaneous action potentials and alter neurotransmission in canine ileum arteries, Circ. Res., 62: 1104-1110.

Smeda J.S., R.M.K.W. Lee and J.B. Forrest, 1988a, Prenatal and postnatal hydralazine treatment does not prevent renal vessel wall thickening in shr despite the absence of hypertension, Circ. Res., 63: 534-542.

Smeda J.S., R.M.K.W. Lee and J.B. Forrest, 1988b, Structural and reactivity alterations of the renal vasculature of spontaneously hypertensive rats prior to and during established hypertension, Circ. Res., 63: 518-533.

Somlyo A.P. and B. Himpens, 1989, Cell calcium and its regulation in smooth muscle, Faseb J., 3: 2266-2276.

Somlyo A.V. and A.P. Somlyo, 1968, Electromechanical and pharmacomechanical coupling in vascular smooth muscle, ]. Pharmacol. Exp. Therap., 159: 129-145.

Stadler E., J.H. Campbell and G.R. Campbell, 1989, Do cultured vascular smooth muscle cells resemble those of the artery wall? If not, why not?, J. Cardiovasc. I'harmacol, 14: SI-S8.

Standen N.B., J.M. Quayle, N.W. Davies, J.E. Brayden, Y. Huang and M.T. Nelson, 1989, Hyperpolarizing vasodilators activate ATP-sensitive $\mathrm{K}^{*}$ channels in arterial smooth muscle, Science, 245: 177-180.

Struijker Boudier H.A.J., J.F.M. Smits and J.G.R. De Mey, 1990, The pharmacology of calcium antagonists: A review, J. Cardiovasc. Pharmacol., 15: S1-S10.

Sturek M. and K. Hermsmeyer, 1986, Calcium and sodium channels in spontaneously contracting vascular muscle cells, Science., 233: 475-478.

Suzuki E., G. Tsujimoto, K. Tamura and K. Hashimoto, 1990, Two pharmacologically distinct $\alpha_{1}$-adrenoceptor subtypes in the contraction of rabbit aorta: each subtype couples with a different $\mathrm{Ca}^{2+}$ signalling mechanism and plays a different physiological role, Mol. Pharmacol., 38: 725-736.

Swales J.D., 1990, Functional disturbance of the cell membrane in hypertension, J. Hypertension., 8: S203-S211.

Tabrichi R. and C.R. Triggle, 1991, Pressor actions of arginine vasopressin in pithed Sprague-Dawley, Wistar Kyoto and spontaneously hypertensive rats before and after treatment with nifedipine or pertussis toxin, J. Hypertension., 9: 813-818.

Takahashi K., K. Hiwada and T. Kokubu, 1988, Vascular smooth muscle calponin, Hypertension., 11: 620-626.

Tesfamariam B. and W. Halpern, 1987, Modulation of adrenergic responses in pressurized resistance arteries by flow, Am. J. Physiol., 253: H1112-H1119. 


\section{REFERENCES}

Triggle C.R., 1989, Reactivity and sensitivity changes of blood vessels in hypertension, in: Blood Vessel Changes in Hypertension: Structure and Function, Lee R.M.K.W., CRC Press Inc., Boca Raton, USA, 25-44.

Triggle C.R. and I. Laher, 1985, A review of changes in vascular smooth muscle functions in hypertension: isolated tissue versus in vivo studies, Can. J. Physiol. Pharmacol., 63: 355-365.

Tung L.H., M.J. Rand and W.J. Louis, 1986, Positive chronotropic responses to cardiac $\alpha_{1}$-adrenoceptor activation in the pithed rat, J. Auton. Pharmacol., 5: 285-290.

Uehara Y., M. Ishii, T. Ishimitsu and T. Sugimoto, 1988, Enhanced phospholipase-C activity in the vascular wall of SHR, Hypertension., 11: 28-33.

Vaghy P.L., G.P. Dube, I.L. Grupp, G. Grupp, J.S. Williams, Y.H. Baik and A. Schwartz, 1985, A proposed pharmacological role for dihydropyridine binding sites in heart and coronary smooth muscle, in: Cardiovascular Effects of Dihydropyridine-type Calcium Antagonists and Agonists, Fleckenstein A., C. Van Breemen, R. Gross and F. Hoffmeister, Springer Verlag, Heidelberg, 156-184.

Van Breemen C. and K. Saida, 1989, Cellular mechanisms regulating $\left[\mathrm{Ca}^{2+}\right]$ smooth muscle, Ann. Rev. Physiol., 51: 315-329.

Van Breemen C., B.R. Farinas, P. Gerba and E.D. Mcnaughton, 1972, Excitation-contraction coupling in rabbit aorta studied by the lanthanum method for measuring cellular calcium influx, Circ. Res, 30: 44.

Van Breemen C., P. Leijten, H. Yamamoto, P. Aaronson and C. Cauvin, 1986, Calcium activation of vascular smooth muscle. State of the art lecture, Hypertension., 8: II89-II95.

Vanhoutte P.M., 1985, Calcium-entry blockers, vascular smooth muscle and systemic hypertension, Am. J. Cardiol., 55: 17b-23b.

Vanhoutte P.M., 1989, Endothelium and control of vascular function, Hypertension., 13: 658-667.

Vanhoutte P.M., T.J. Verbeuren and R.C. Webb, 1981, Local modulation of adrenergic neuro effector interaction in the blood vessel wall, Physiol. Rev., 61: 151-218.

van Kleef E.M., J.F.M. Smits, J.G.R. De Mey, J.P.M. Cleutjens, D.M. Lombardi, S.M. Schwartz and M.J.A.P. Daemen, 1992, $\alpha_{1}$-Adrenoceptor blockade reduces the angiotensin II-induced vascular smooth muscle cell DNA synthesis in the rat thoracic aorta and carotid artery, Circ. Res., 70: 1122-1127.

van Zwieten P.A., (ed.), 1989, Clinical Aspects of Calcium Entry Blockers, Karger, Basel, Switzerland, 200.

Wallenstein S., C.L. Zucker and J.L. Fleiss, 1980, Some statistical methods useful in circulation research, Circ. Res., 47: 1-9.

Warshaw D.M., D.T. Root and W. Halpern, 1980, Effects of antihypertensive drug therapy on the morphology and mechanisms of resistance arteries from SHR, Blood. Vessels., 17: $257-270$.

Watson P.A., 199l, Function follows from: generation of intracellular signals by cell deformation, Faseb. J., 5: 2013-2019

Webb R.C., 1984, Vascular changes in hypertension, in: Cardiovascular Pharmacology, Antonaccio M., Raven Press, New York, 215-255.

Weston A.H. and A. Abbot, 1987, New class of antihypertensive acts by opening $\mathrm{K}^{+}$channels, TIPS, 8: 283-284.

Wilson K.M. and K.P. Minneman, 1990, Different pathways of ${ }^{3} \mathrm{H}$ inositol phosphate formation mediated by $\alpha_{1 A}$-adrenergic and $\alpha_{i B}$-adrenergic receptors, J. Biol. Chem., 265: 17601-17606.

Winquist R.J., P.B. Bunting, E.P. Baskin and A.A. Wallace, 1984, Decreased endotheliumdependent relaxation in New Zealand genetic hypertensive rats, J. Hypertension., 2: 541-545.

Yamatani T., A. Yamaguchi, A. Nakamura, T. Morishita, S. Kadowaki, T. Fujita and T. Chiba, 1990, Activation of PKC inhibits NAF-induced inositol phospholipid turnover in rat insulinoma cells, Am. J. Physiol, 259: E73-E79. 


\section{REFERENCES}

Yanagisawa M., H. Kurihara, S. Kimura, Y. Tomobe, M. Kobayashi, Y. Mitsui, Y. Yazaki, K. Goto and T. Masaki, 1988, A novel potent vasoconstrictor peptide produced by vascular endothelial cells, Nature., 332: 411-415.

Yatani A., C.L. Seidel, J. Allen and A.M. Brown, 1987, Whole-cell and single-channel calcium currents of isolated smooth muscle cells from saphenous vein, Circ. Res., 60: 523-533.

Zelis R. and R. Moore, 1989, Recent insights into the calcium channel, Circulation., 80: IV14 -IV16.

Zeng Y.Y., C.G. Benishin and P.K.T. Pang, 1989, Guanine nucleotide binding proteins may modulate gating of calcium channels in vascular smooth muscle. I Studies with guanosine

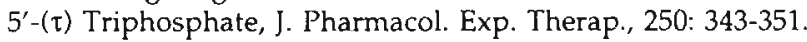




\section{SUMMARY}

Total vascular resistance is controlled by the functional and structural regulatory capacity of resistance-sized arteries. Vascular reactivity depends on the function of excitation-contraction coupling mechanisms in the vascular smooth muscle cells and on the structure of the vessel wall. Furthermore, remote (neuro-endocrine) and local (endothelium, metabolic and mechanical factors) systems modulate vascular function acutely and vascular structure chronically. Alterations in the reactivity of resistance arteries may lead to the development and maintenance of high blood pressure. However, the mechanisms responsible for these changes are as yet, unknown.

In the present dissertation we investigated excitation-contraction coupling mechanisms of small artery smooth muscle and evaluated the role of these in the development and maintenance of hypertension. Small arteries are different from large conducting arteries with respect to their susceptibility to calcium antagonists. Heterogenous distribution of $\alpha_{1}$-adrenoceptor subtypes over large and small arteries may contribute to this regional difference (Chapter 3). Furthermore, effects of dihydropyridines in small arteries indicate that during pharmacological stimulation, voltage operated calcium channels (VOC) contribute to a larger extent to the contractile response in small than in large arteries. Observations with dihydropyridine calcium antagonists and agonists on contractile responses induced by high potassium and noradrenaline suggest that the function of VOC may be modulated directly by noradrenaline (Chapter 4).

Effects of $\alpha_{1}$-adrenoceptor activation on calcium channels was found to be mediated by pertussis toxin-sensitive G-proteins. Also $\alpha_{1}$-adrenoceptor mediated release of intracellular calcium was sensitive to pertussis-toxin suggesting a role for G-proteins in the activation of phospholipase-C (PLC) and subsequent generation of inositol triphosphate $\left(\mathrm{IP}_{3}\right)$ (Chapter 5). Activation of PLC normally liads also to the generation of diacylglycerol which in turn activates protein kinase- $C$ (PKC). Stimulation of this kinase with a phorbol ester and inhibition of the enzyme affected contractile responses to electromechanical stimulation. The data suggest that PKC may affect both the function of VOC and the sensitivity of the contractile apparatus for calcium (Chapter 6).

Distension of small arteries affected the sensitivity and reactivity to potassium, noradrenaline and calcium (Chapter 7). This hypersensitivity can be attributed to effects of distension on smooth muscle membrane potential $\left(V_{m}\right)$, intracellular calcium concentrations $\left(\left[\mathrm{Ca}^{2+}\right]_{i}\right)$ and on the sensitivity of the contractile apparatus for calcium (Chapter 8). Effects of distension were comparable to those observed during activation of PKC. The hypersensitivity to extracellular calcium during stimulation with noradrenaline that is observed in small arteries of spontaneously hypertensive rats (SHR) may be explained by an altered modulatory effect of noradrenaline on VOC (Chapter 4).

In the final chapters, we studied the relationship of altered vascular sensitivity to the development and maintenance of hypertension in the SHR. Hypersensitivity to vasoconstrictors in small arteries was readily lost during tissue culture, suggesting that hypersensitivity developed in response to the elevated 
blood pressure (Chapter 9). In small arteries of young SHR hyperreactivity to vasoconstrictors, but no hypersensitivity was observed. The alterations could be attributed to increased media mass. Infusion of young normotensive Wistar Kyoto rats with pressor doses of angiotensin II and phenylephrine had differential effects on vascular reactivity and structure. Those observed in rats treated with angiotensin Il were comparable to those seen in young SHR. From these we conclude that in SHR, angiotensin II rather than increases in blood pressure or hypersensitivity to vasoconstrictors may account for alterations in structure and hence hyperreactivity of small arteries (Chapter 10).

Taken together, observations presented in this thesis suggest that different means of excitation-contraction coupling in small arteries interdigitate. These mechanisms may interact at common sites such as the activity of PKC and regulation of $V_{m}$, which in turn affect the function of VOC. In spontaneously hypertensive rats the modulatory role of PKC may be altered during the established phase of high blood pressure resulting in hypersensitivity to vasoconstrictors. However, hypersensitivity to vasoconstrictors or calcium are probably not responsible for the development of hypertension. Changes in vascular structure may precede changes in vascular function in the genesis of hypertension. 


\section{SAMENVATTING}

De weerstand die het bloed ondervindt in de vaatboom wordt gereguleerd door functionele (spiervernauwing of verwijding) en structurele eigenschappen (wanddikte, stijfheid) van kleine arteriën (kleine slagaders). De vasculaire reactiviteit (contractiele functie $=$ vernauwende of verwijdende capaciteit) is afhankelijk van de werking van spiercellen in de vaatwand en van de structuur van het vat. Besturingssystemen zoals zenuwen en hormonen of lokale mechanismen zoals het endotheel (cellaag die de binnenkant van het vat bekleedt), metabole (afvalstoffen), of mechanische (druk, rek) factoren kunnen de vasculaire functie en structuur acuut dan wel op de langere termijn reguleren (Fig. 1.1). Veranderingen in de functie van spiercellen in de wand van kleine arteriën zouden kunnen leiden tot een verhoogde bloeddruk. De mechanismen die hiervoor verantwoordelijk kunnen zijn, zijn echter nog vrijwel onbekend.

In dit proefschrift zijn mechanismen onderzocht die stimulatie van arteriële spiercellen koppelen met krachtontwikkeling. De veranderingen hierin zouden een rol kunnen spelen in het ontstaan van hoge bloeddruk. Samentrekken (contractie) van spiercellen in de vaatwand komt tot stand wanneer een boodschapper (bijvoorbeeld gegeven door een zenuw) zich vasthecht aan een eiwit (receptor) op de celmembraan. Deze receptor stuurt een tweede signaal in de cel dat uiteindelijk via een keten van enzymen tot contractie leidt (Fig. 1.2 en $1.3_{\mathrm{A}}$ ). Bij dit proces is calcium essentieel. Wanneer de cel gestimuleerd wordt stroomt calcium de cel binnen door kanalen die op verschillende manieren geopend kunnen worden. De resultaten in hoofdstuk 3 tonen aan dat kleine arterien verschillen van grote omdat ze veel gevoeliger zijn voor calcium antagonisten die de instroom van calcium belemmeren. De oorzaak hiervoor zou een verschil in verdeling van specifieke receptoren ( $\alpha_{1}$-adrenoceptoren) kunnen zijn over grote en kleine vaten. Verder blijkt, uit de resultaten verkregen met blokkers van calciumkanalen (Hoofdstuk 4) dat in kleine vaten verschillende manieren van stimulatie (via een receptor of door verandering van membraan potentiaal = elektrisch spanningsverschil over de celmembraan) niet zo sterk van elkaar gescheiden zijn als werd gedacht. Verschillende calciumkanalen zouden wel eens één en dezelfde kunnen zijn (Hoofdstuk 4).

$\alpha_{1}$-Adrenoceptoren blijken gekoppeld te zijn aan zogenaamde G-eiwitten die op hun beurt calcium kanalen kunnen openen of die, uit opslagplaatsen in de cel, calcium kunnen vrijmaken via activatie van een bepaalde enzymketen (Hoordstuk 5). Een enzym, het fosfolipase-C, breekt een vetsuiker in tweeën. De stoffen die overblijven, inositol trifosfaat en diacylglycerol, zullen op hun beurt calcium in de cel vrijmaken of een volgend enzym activeren, het proteine kinase-( $(P K C)$ (Fig. 1.3. $)$. Resultaten die verkregen zijn met behulp van een activator of een remmer van PKC suggereren dat dit enzym een positief effect zou kunnen hebben zowel op de opening van calciumkanalen als op de calciumgevoeligheid van het contractiel apparaat (verzameling eiwitten verantwoordelijk voor contractie van de cel) (Hootdstuk o). 
Vergroten van de rek op spiercellen in kleine vaten beinvloedt de gevoeligheid voor verschillende prikkels (Hoofdstuk 7). Deze verhoogde gevoeligheid voor vaatvernauwers kan veroorzaakt worden door een effect van rek up de membraan potentiaal, de hoeveelheid calcium in de cel of de gevoeligheid van het contractiel apparaat voor calcium (Hoofdstuk 8). Opmerkelijk is dat effecten van rek vergelijkbaar zijn met de effecten na activatie van PKC. De verhoogde gevoeligheid voor calcium tijdens receptorstimulatie met de zenuwboodschapper noradrenaline die waargenomen kunnen worden in kleine arteriën van spontaan hypertensieve ratten (SHR; ratten die spontaan een hoge bloeddruk ontwikkellen) zou verklaard kunnen worden door een effect van noradrenaline op calciumkanalen die ook gevoelig zijn voor veranderingen in membraan potentiaal (Hoordstuk 4).

In de laatste hoofdstukken staat een onderzoek beschreven naar de relatie tussen veranderingen in de gevoeligheid van vasculaire gladde spiercellen voor verschillende stimuli en het ontstaan en onderhouden van een hoge bloeddruk in de SHR. Wanneer uit de SHR geïsoleerde kleine arteriën in cultuur (weefselkweek) gebracht worden (d.w.z. in de afwezigheid van een verhoogde bloeddruk), dan verdwijnt de toegenomen gevoeligheid voor contractiele stimuli. Dit suggereert dat het verschil in gevoeligheid voor vaatvernauwers in vaten uit normale dieren en dieren met een hoge bloeddruk een gevolg zou kunnen zijn van de verhoogde bloeddruk (hoofdstuk 9). Verhogen van de bloeddruk in ratten met een normale bloeddruk met behulp van verschillende vaatvernauwers heeft verschillende effecten op kleine arteriën. Wanneer angiotensine II (hormoon betrokken o.a. bij bloeddrukregulatie) als bloeddruk-verhogende stof wordt gebruikt, dan treden veranderingen op die vergelijkbaar zijn met die in vaten van jonge SHR. Uit deze resultaten kan geconcludeerd worden dat in de SHR waarschijnlijk angiotensine II, eerder dan een toegenomen bloeddruk of een verandering in de gevoeligheid van kleine arterièn, verantwoordelijk zou kunnen zijn voor structurele veranderingen en als gevolg daarvan veranderingen in de contractiekracht van deze vaten (Hoofdstuk 10).

Kort samengevat suggereren de resultaten die beschreven zijn in dit proefschrift dat verschillende manieren van stimulatie van kleine arteriën leidt tot krachtontwikkeling via mechanismen die elkaar beïnloeden via de activiteit van PKC of de membraan potentiaal (Fig. 11.3). De laatste beinvloedt op zijn beurt de potentiaal gevoelige calcium kanalen in de celmembraan. Bij de SHR zou een verandering in de rol van PKC als gevolg van de aanhoudende hoge bloeddruk kunnen leiden tot de waargenomen overgevoeligheid in kleine arteriën. Echter, de overgevoeligheid voor vaatvernauwers of calcium is waarschijnlijk niet verantwoordelijk voor de ontwikkeling van hoge bloeddruk. Vermoedelijk zijn veranderingen in de structuur van kleine arterien betrokken bij het ontstaan van hoge bloeddruk. 


\title{
CURRICULUM VITAE
}

\author{
Harrie C.M. Boonen
}

23 januari 1964

1976-1981

$1981-1985$

1985

1985-1987

1987-1988

1988-1992

1993-
Geboren te Buggenum

HAVO, scholengemeenschap St. Ursula te Horn

Opleiding analist HLO-Biologie, Dr. ir. W.L. Ghijsen instituut te Utrecht

Onderzoeks-assistent op het laboratorium voor Farmacologie, Rijksinstituut voor Volksgezondheid en Milieuhygiëne te Utrecht

\section{Dienstplichtig onderofficier}

Biologisch onderzoeks-assistent, vakgroep Farmacologie, Faculteit der Geneeskunde, Rijksuniversiteit Limburg, Maastricht

Assistent in Opleiding, in dezelfde vakgroep

Post-doctoraal medewerker, in dezelfde vakgroep

\section{LIST OF PUBLICATIONS}

\section{Full papers}

Asbeck van B.S., F.C. Hillen, H.C.M. Boonen, Y. de Jong, J.A.M.A. Dormans, M.A.A van der Wal, J.J.M. Marx and B. Sangster, 1989, Continuous intravenous infusion of deferoxamine reduces mortality by paraquat in vitamin E-deficient rats, Am. Rev. Respir. Dis., 139: 769773.

Boonen H.C.M. and J.G.R De Mey, 1990, Effects of tertatolol on the responsiveness of isolated femoral, mesenteric, and renal resistance arteries to adrenergic stimuli, J. Cardiovasc. Pharmacol. 15: 124-129.

Boonen H.C.M. and J.G.R. De Mey, 1990, Increased calcium sensitivity in isolated resistance arteries from spontaneously hypertensive rats: effects of dihydropyridines, Eur. J. Pharmacol. 179: 403-412.

Boonen H.C.M. and J.G.R. De Mey, 1990, G-proteins are involved in contractile responses of isolated mesenteric resistance arteries to agonists, Naunyn Schmiedeberg's Arch. Pharmacol. 342: $462-468$.

Bownen H.C.M. and J.G.R. De Mey, 1991, Effects of a phorbol ester and staurosporin on electro- and pharmacomechanical coupling in a resistance artery., Eur. J. Pharmacol. 202: 25-32. 
Boonen H.C.M., P.M.H. Schiffers, G.E. Fazzi, G.M.J Janssen, M.J.A.P. Daemen and J.G.R. De Mey, 1991, DNA synthesis in isolated arteries. Kinetics and structural consequences. Am. J. Physiol. 260: H210-H217.

Boonen H.C.M., P.E. Jensen, M.]. Mulvany, C. Aalkjaer and I.G.R. De Mey, 1992, Distension sensitizes small artery smooth muscle to vasoconstrictors and intracellular calcium. in preparation.

Boonen H.C.M., and J.G.R. De Mey, 1992, $\alpha_{1}$-Adrenoceptor subtype population and calcium antagonist efficacy in large and small mesenteric arteries of the rat. in preparation.

Boonen H.C.M., D.H.F. Pelzer and J.G.R. De Mey, 1992, Loss of hypersensitivity to vasocon strictors in mesenteric muscular and resistance arteries of spontaneously hypertensive rats following tissue culture. in preparation.

Boonen H.C.M., M.J.A.P. Daemen, P.A. Eerdmans, G.E. Fazzi, G.M.J. Janssen, E.M. van Kleef, P.M.H. Schiffers and J.G.R. De Mey, 1992, Mesenteric resistance artery changes following vasoconstrictor infusion in young rats. submitted.

De Mey J.G.R., H.C.M. Boonen and H.A.J. Struijker Boudier, 1988, Rhythmic contractile activity in resistance arteries of spontaneously hypertensive rats. in: Resistance arteries, eds. Halpern et al., Perinatology press, Ithaca, New York, USA, pp. 336-341.

De Mey J.G.R., M.P Uitendaal, H.C.M. Boonen, M.J.J.F. Vrijdag, M.J.A.P. Daemen and H.A.J. Struijker Boudier, 1989. Acute and long-term effects of tissue culture on contractile reactivity in renal arteries of the rat, Circ. Res. 65: 1125-1135.

De Mey J.G.R., M.P. Uitendaal, H.C.M. Boonen and M.J.J.F. Vrijdag, 1990, Acute and long term effects of 5-hydroxytryptamine in isolated renal arteries in vitro, in: Cardiovascular pharmacology of 5-hydroxytryptamine, eds. Saxena et al., Kluwer, Dordrecht, the Netherlands, Pp. 339-342.

De Mey J.G.R., H.C.M. Boonen, P.M.H. Schiffers, G.E. Fazzi and G.M.J Janssen, 1991, What could underlie hyperplasia of resistance arteries in hypertension?, in: Resistance arteries. structure and function. eds. Mulvany et al. Elsevier, Amsterdam, the Netherlands, pp. 289291.

De Mey J.G.R., M.J.A.P. Daemen, H.C.M. Boonen, F.T. Busman, E.H. Dijkstra, G.E. Farzi, G.M.J. Janssen, P,M,H, Schiffers, H.A.J. Struijker Boudier and M,J,J,F, Vrijdag, 1991, In vivo synthesis is not uniformly increased in arterial smooth muscle of young spontaneously hypertensive rats, J. Hypertension, 9: 695-701.

De Mey J.G.R, M.P. Uitendaal, H.C.M. Boonen, P.M.H. Schiffers and G.E. Fazzi, 1991, Growth responses in isolated elastic, muscular and resistance-sized arterial segments of the rat, Blood Vessels, 28: 372-385.

\section{Abstracts}

Boonen H.C.M. and J.G.R. De Mey, 1989, Effects of pertussis toxin on contractile responses to agonists and calcium in isolated mesenteric resistance sized arteries, Pharmaceutisch Weekblad Scientific edition, 11(6): J3

Boonen H.C.M. and J.G.R De Mey, 1989, Loss of hypersensitivity in mesenteric muscular and resistance arteries of spontaneously hypertensive rats following short term tissue culture, $6^{\text {th }}$ Ann. Sym. SHR, IOWA, USA,

Boonen H.C.M. and J.G.R De Mey, 1989, Effects of tertatolol on the contractile reactivity of isolated resistance arteries, Arch. Int. Pharmacodyn.

Boonen H.C.M., D.H.F Pelzer and J.G.R De Mey, 1989, Effects of short-term tissue culture on the contractile reactivity of mesenteric resistance-sized arteries, Clin. Exp. Pharmacol., 3: 437.

Boonen H.C.M., D.H.F. Pelzer and H.A.J. Strujjker Boudier, 1989, Felodipine affects contractile reactivity in muscular and resistance arteries with different potency and selectivity. Naunyn Schmiedeberg's Arch. Pharmacol, 339: 185

Boonen H.C.M., H.A.J. Struijker Boudier and J.G.R De Mey, 1989, Tissue culture abolishes hypersensitivity of muscular and resistance arteries of spontaneously hypertensive rats, Dutch Hypertension Association, Utrecht, the Netherlands. 
